\title{
C-CN Bond Cleavage using Palladium Supported by a Dippe Ligand
}

Lloyd Munjanja ${ }^{\ddagger}$, Coralys Torres-López $\$$ William W. Brennessel and William D. Jones*

Department of Chemistry, University of Rochester, Rochester, New York 14627, United States

\section{Supporting Information}

\section{Contents:}

\begin{tabular}{|l|l|}
\hline General Experimental Information & S2 \\
\hline $\begin{array}{l}\text { Synthesis and Characterization data for the Complexes 2a, 2a', } \\
\text { 3a', 3b }\end{array}$ & S3-S24 \\
\hline X-ray Structures for 2a, 2a', 3a', and 3b & S25-S115 \\
\hline References & S116 \\
\hline
\end{tabular}


General Considerations. All operations and routine manipulations were performed under a nitrogen atmosphere; either on a high-vacuum line using modified Schlenk techniques or in a Vacuum Atmospheres Corp. Dri-Lab. THF- $d_{8}$ was purchased from Cambridge Isotope

Laboratories, Inc. Prior to use THF- $d_{8}$ was distilled under vacuum from a dark purple solution of benzophenone ketyl and stored in an ampoule with a Teflon valve. Acetonitrile and benzonitrile was purchased from Sigma Aldrich. Acetonitrile was dried using $\mathrm{CaH}_{2}$ and stored under molecular sieves.

All ${ }^{1} \mathrm{H}^{13} \mathrm{C},{ }^{31} \mathrm{P},{ }^{11} \mathrm{~B}$ NMR spectra were collected on either a Bruker Avance 400 or Avance 500 $\mathrm{MHz}$ spectrometer. All chemical shifts were reported in ppm $(\delta)$ relative to tetramethylsilane and referenced to the chemical shifts of residual solvent resonances $\left(\mathrm{C}_{6} \mathrm{HD}_{5}, \delta 7.16\right.$ or $\left.\delta 128.0\right)$. While ${ }^{1} \mathrm{H}$ chemical shifts are given to 3 decimal places $( \pm 0.4 \mathrm{~Hz})$, these values can vary slightly with concentration and temperature. ${ }^{13} \mathrm{C}$ shifts are given to 2 decimal places $( \pm 1 \mathrm{~Hz}) .{ }^{31} \mathrm{P}\left\{{ }^{1} \mathrm{H}\right\}$ NMR spectra were referenced relative to external $\mathrm{H}_{3} \mathrm{PO}_{4} \cdot{ }^{11} \mathrm{~B}\left\{{ }^{1} \mathrm{H}\right\}$ was referenced relative to boron trifluoride diethyl etherate. Elemental analysis was performed by Dr. William W. Brennessel at the University of Rochester using a Perkin Elmer 2400 Series II elemental analyzer in $\mathrm{CHN}$ mode. Infrared spectra were recorded in the solid state on a Thermo Scientific Nicolet 4700 FT-IR spectrometer equipped with smart orbit diamond attenuated total reflectance (ATR) accessory. All photolysis were performed using an Oriel $200 \mathrm{~W} \mathrm{Hg}(\mathrm{Xe})$ arc lamp fitted with a water-filled IR Filter. 
Preparation of complex 2a, (dippe)Pd(CH$\left(\mathrm{CH}_{3} \mathrm{CN}-\mathrm{BEt}_{3}\right)$, in situ: Under a nitrogen atmosphere, $\mathrm{KHBEt}_{3}(91 \mu \mathrm{L}$ of $1 \mathrm{M}$ solution in THF, $0.091 \mathrm{mmol})$ was added to a stirred suspension of [(dippe) $\left.\mathrm{PdCl}_{2}\right](20 \mathrm{mg}, 0.046 \mathrm{mmol})$ in $0.6 \mathrm{~mL}$ of THF- $d_{8}$. The resulting intense dark red solution was then filtered through Celite. The filtrate was transferred to a J. Young NMR tube after which an excess of acetonitrile $(0.1 \mathrm{~mL})$ was added. Release of $\mathrm{H}_{2}$ gas is observed while the color of the solution lightens up to transparent pale yellow. Due to 2a being stable in solution and decomposing under vacuo we characterized it in situ. ${ }^{1}$

${ }^{31} \mathrm{P}\left\{{ }^{1} \mathrm{H}\right\}$ NMR $(162 \mathrm{MHz}, \mathrm{THF}) \delta 75.55\left(\mathrm{~d},{ }^{2} J_{\mathrm{P}-\mathrm{P}}=25.0 \mathrm{~Hz}\right), 61.70\left(\mathrm{~d},{ }^{2} J_{\mathrm{P}-\mathrm{P}}=25.0 \mathrm{~Hz}\right)$.

${ }^{1} \mathrm{H}$ NMR (400 MHz, THF) $\delta 2.66\left(\mathrm{~d},{ }^{4} \mathrm{~J}_{\mathrm{H}-\mathrm{P}}=6.2 \mathrm{~Hz}, 3 \mathrm{H}, \mathrm{CH}_{3} \mathrm{CN}\right)$

${ }^{13} \mathrm{C}$ NMR $(101 \mathrm{MHz}, \mathrm{THF}) \delta 149.70\left(\mathrm{dd},{ }^{2} J_{\mathrm{C}-\mathrm{P}}=65.6,3.5 \mathrm{~Hz}, \mathrm{CN}\right), 23.02(\mathrm{dd}, J=22.5,16.7 \mathrm{~Hz}$, $\mathrm{CH}), 21.56\left(\mathrm{dd}, J=19.6,13.5 \mathrm{~Hz}, \mathrm{CH}_{2}\right), 20.47\left(\mathrm{dd}, J=13.0,8.8 \mathrm{~Hz}, \mathrm{CH}_{2}\right), 19.32\left(\mathrm{~s}, \mathrm{CH}_{3}\right), 19.11$ $\left(\mathrm{s}, \mathrm{CH}_{3}\right) .{ }^{11} \mathrm{~B}\left\{{ }^{1} \mathrm{H}\right\} \mathrm{NMR}(128 \mathrm{MHz}, \mathrm{THF}) \delta-13.15\left(\mathrm{~s}, \mathrm{CN} B \mathrm{Et}_{3}\right)$.

Solution IR (ATR, $\left.\mathrm{cm}^{-1}\right): 1811\left(v_{\mathrm{CN}}\right)$ 


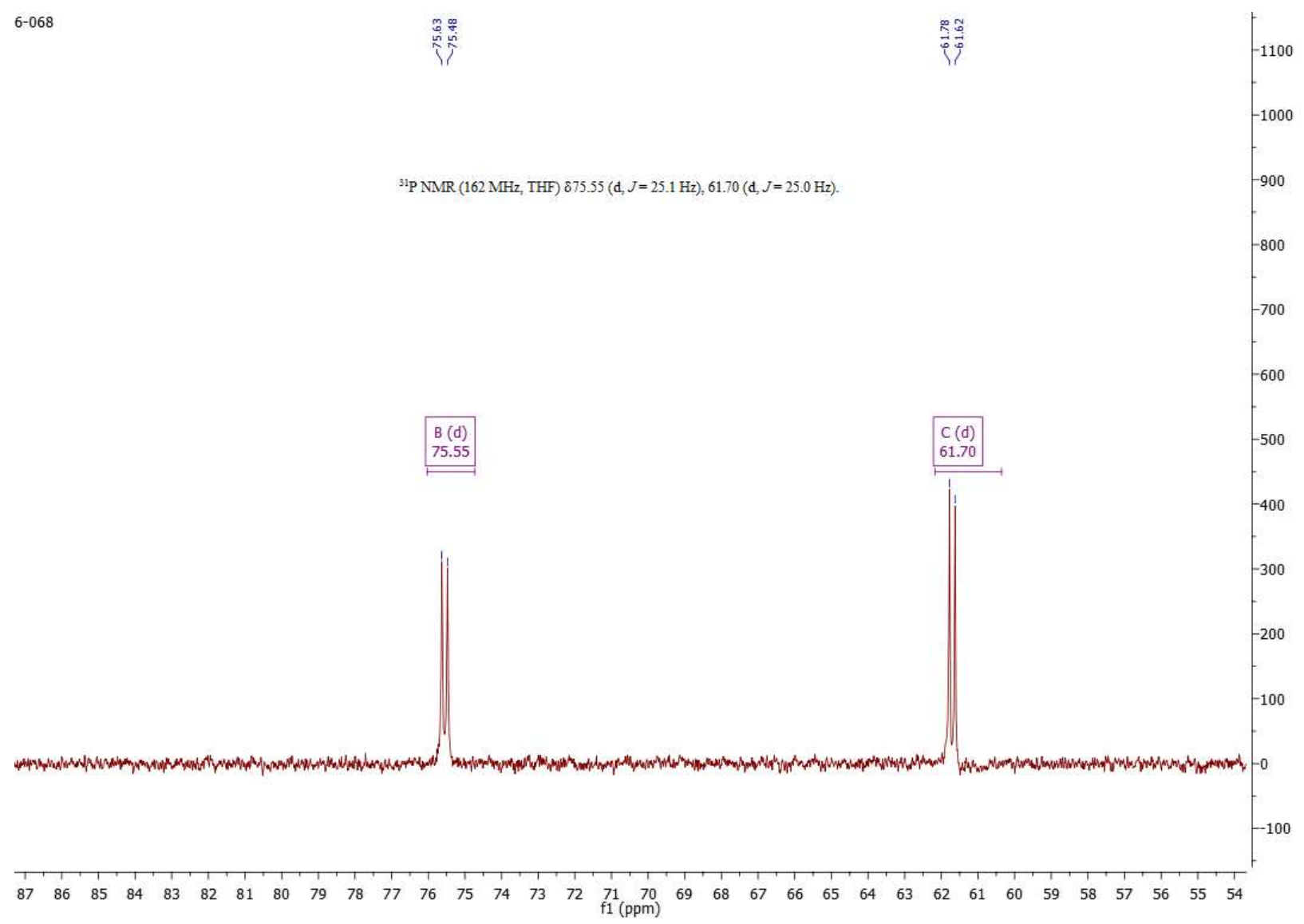

Figure S1: ${ }^{31} \mathrm{P}\left\{{ }^{1} \mathrm{H}\right\}$ NMR of $\mathbf{2 a}$ in THF 


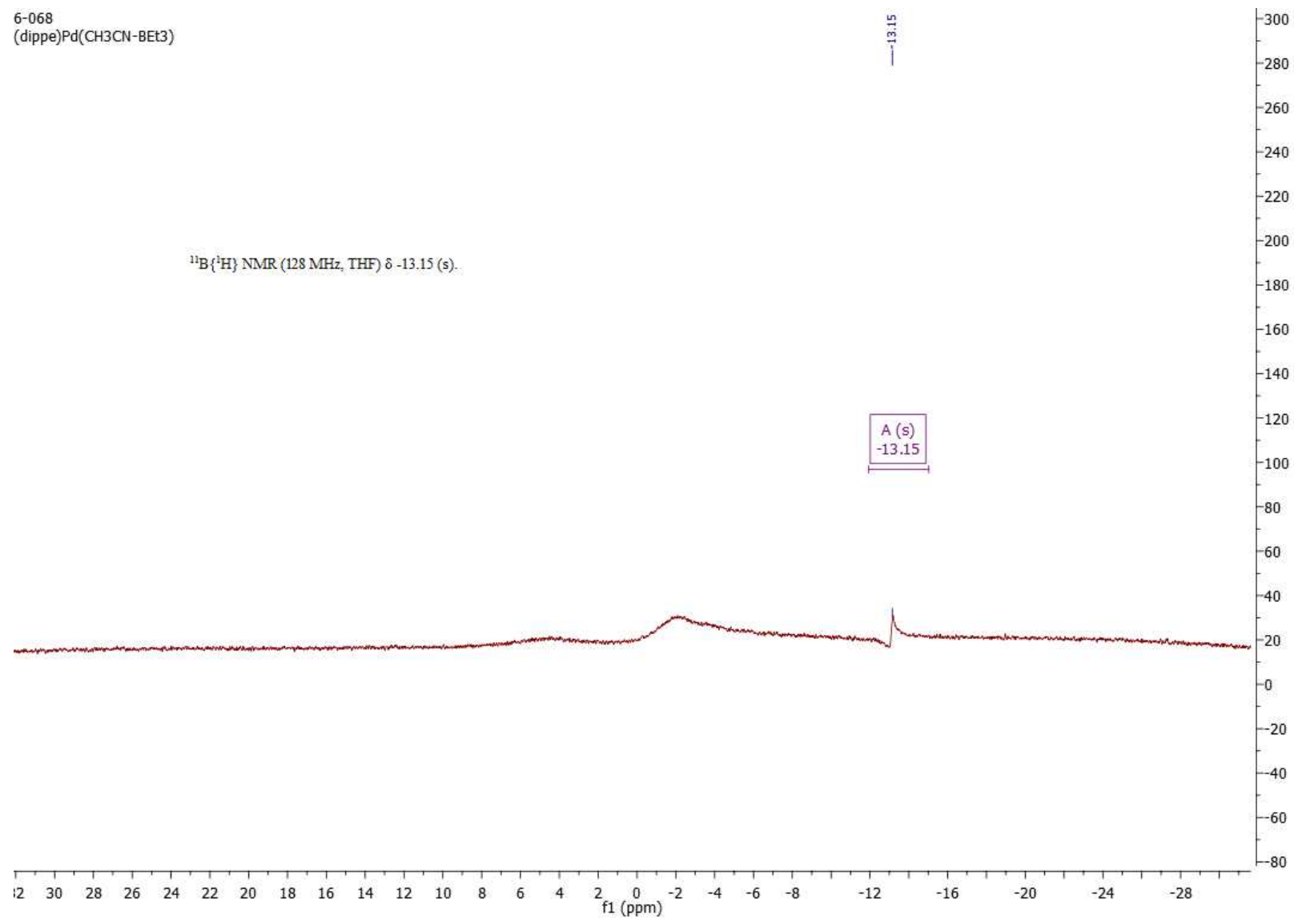

Figure S2: ${ }^{11 \mathrm{~B}}\left\{{ }^{1} \mathrm{H}\right\}$ NMR of $\mathbf{2 a}$ in THF 


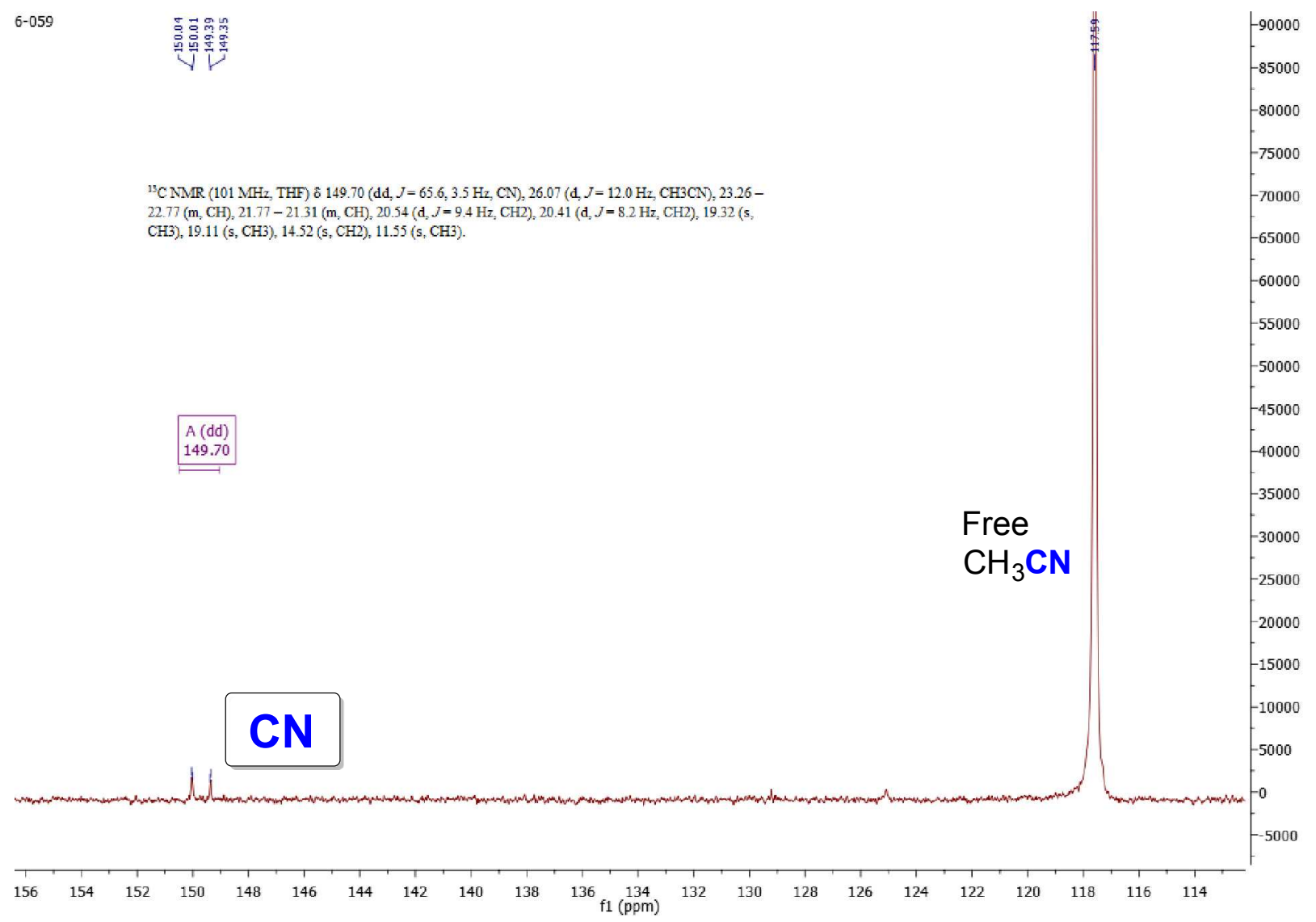

Figure S3: ${ }^{13 \mathrm{C}}\left\{{ }^{1} \mathrm{H}\right\}$ NMR of $\mathbf{2 a}$ in THF (CN region) 


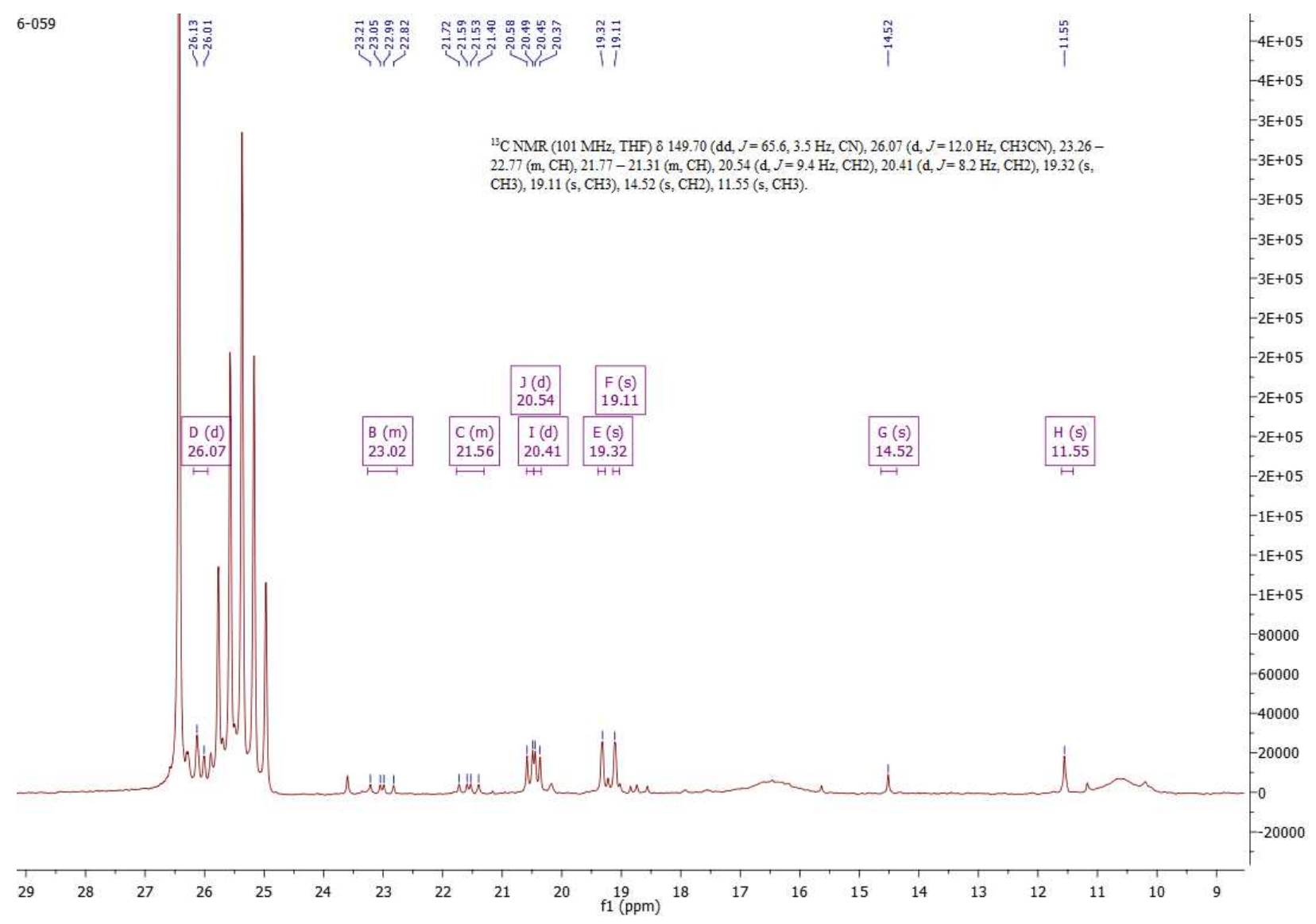

Figure S4: ${ }^{13 \mathrm{C}}\left\{{ }^{1} \mathrm{H}\right\}$ NMR of $\mathbf{2 a}$ in THF (aliphatic region) 


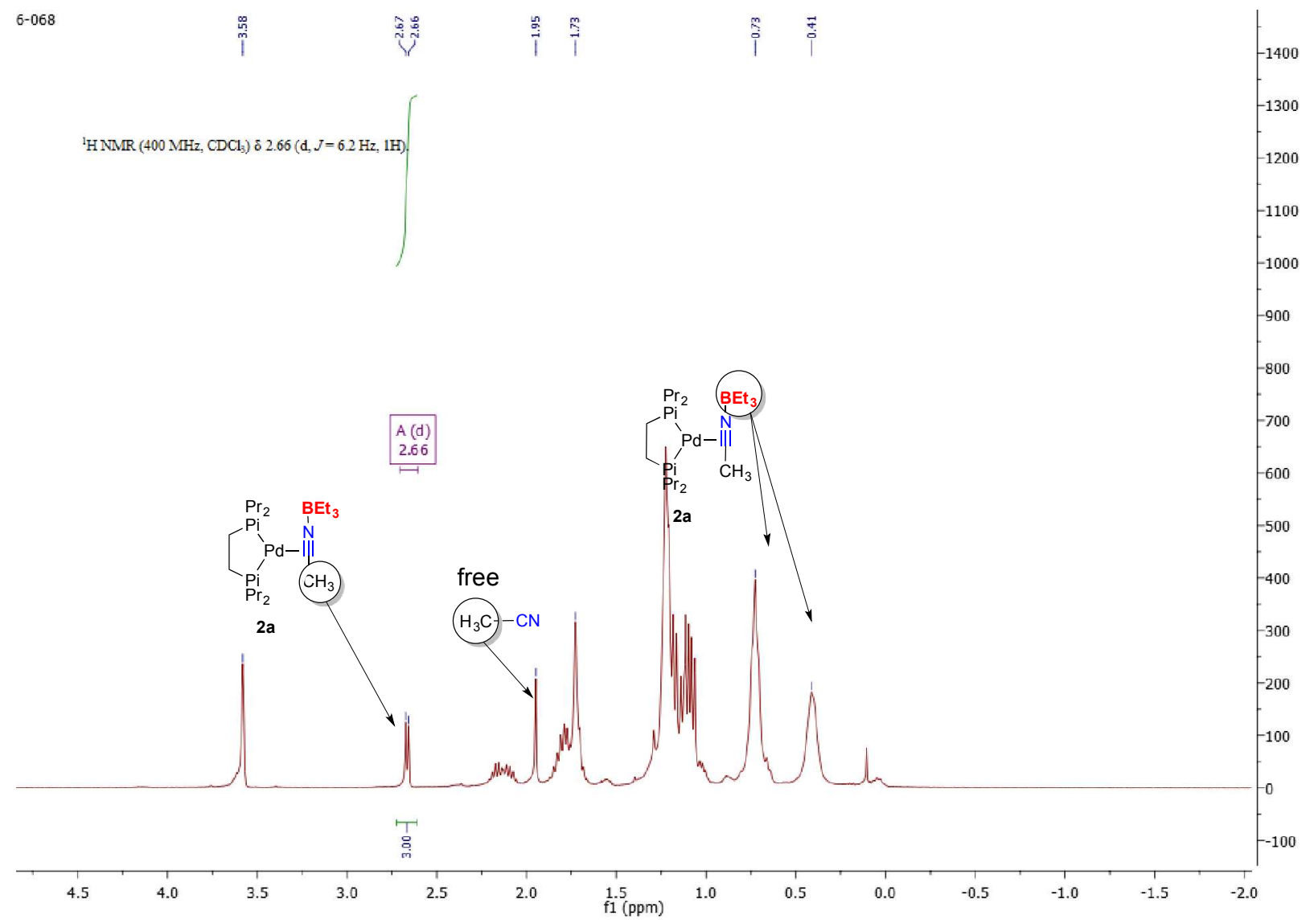

Figure S5: ${ }^{1} \mathrm{H}$ NMR of 2a in THF (aliphatic region) 
Preparation of complex 2a' (dippe)Pd( $\left.\mathrm{CH}_{3} \mathrm{CN}-\mathrm{BPh}_{3}\right)$ : Under a nitrogen atmosphere, $\mathrm{KHBEt}_{3}$ ( $0.9 \mathrm{~mL}$ of $1 \mathrm{M}$ solution in THF, $0.9096 \mathrm{mmol}$ ) was added to a stirred suspension of [(dippe) $\mathrm{PdCl}_{2}$ ] (200 mg, $\left.0.4548 \mathrm{mmol}\right)$ in $3.0 \mathrm{~mL}$ of toluene. The resulting intense dark red solution was then filtered through Celite before adding $0.5 \mathrm{~mL}$ of $\mathrm{CH}_{3} \mathrm{CN}$ to the filtrate. After the solution stopped giving off $\mathrm{H}_{2}$ gas, $\mathrm{BPh}_{3}(220 \mathrm{mg}, 0.9096 \mathrm{mmol})$ was added and the solution stirred for $5 \mathrm{~min}$. The pale yellow solution was dried on vacuo to give a light red oil. The oil was dissolved in $0.1 \mathrm{~mL}$ of THF and $10 \mathrm{~mL}$ of pentane to wash of excess $\mathrm{BEt}_{3}$ and $\mathrm{BPh}_{3}$. This process was repeated three times until a pale yellow solid crushed out in solution. The pale yellow solid was dried in vacuo to yield 2a' (222 $\mathrm{mg}, 75 \%$ ) ${ }^{31} \mathrm{P}\left\{{ }^{1} \mathrm{H}\right\}$ NMR $\left(162 \mathrm{MHz}, \mathrm{THF}-d_{8}\right) \delta 76.81\left(\mathrm{~d},{ }^{2} J_{\mathrm{P}-\mathrm{P}}=19.9 \mathrm{~Hz}\right), 60.02\left(\mathrm{~d},{ }^{2} J_{\mathrm{P}-\mathrm{P}}=19.9 \mathrm{~Hz}\right) ;$ ${ }^{1} \mathrm{H}$ NMR $\left(400 \mathrm{MHz}, \mathrm{THF}-d_{8}\right) \delta 7.35(\mathrm{~d}, J=6.9 \mathrm{~Hz}, 6 \mathrm{H}, \operatorname{Ar} H), 7.00(\mathrm{t}, J=7.3 \mathrm{~Hz}, 6 \mathrm{H}, \operatorname{Ar} H)$, $6.93(\mathrm{~d}, J=7.2 \mathrm{~Hz}, 3 \mathrm{H}, \mathrm{Ar} H), 2.23-2.10(\mathrm{~m}, 2 \mathrm{H}, \mathrm{CH}), 2.08\left(\mathrm{~d},{ }^{4} J_{\mathrm{H}-\mathrm{P}}=6.3 \mathrm{~Hz}, 3 \mathrm{H}, \mathrm{CH}_{3} \mathrm{CN}\right)$, $1.98-1.77\left(\mathrm{~m}, 6 \mathrm{H},\left\{2 \mathrm{H} \mathrm{CH}\right.\right.$ and $\left.\left.4 \mathrm{H} \mathrm{CH}_{2}\right\}\right), 1.25-1.07\left(\mathrm{~m}, 12 \mathrm{H}, \mathrm{CH}_{3}\right), 0.95(\mathrm{dd}, J=13.0,6.9$ $\left.\mathrm{Hz}, 6 \mathrm{H}, \mathrm{CH}_{3}\right), 0.78\left(\mathrm{dd}, J=16.5,7.1 \mathrm{~Hz}, 6 \mathrm{H}, \mathrm{CH}_{3}\right) ;{ }^{13} \mathrm{C}\left\{{ }^{1} \mathrm{H}\right\}$ NMR $\left(101 \mathrm{MHz}, \mathrm{THF}-d_{8}\right) \delta 156.38$ - $154.31(\mathrm{~m}, \mathrm{CN}), 135.19(\mathrm{~s}, \mathrm{Ar}), 130.56\left(\mathrm{t}, J_{\mathrm{C}-\mathrm{B}}=3.3 \mathrm{~Hz}, \mathrm{Ar}\right), 126.66(\mathrm{~s}, \mathrm{Ar}), 124.39(\mathrm{~s}, \mathrm{Ar})$, $26.23\left(\mathrm{~d},{ }^{3} J_{\mathrm{C}-\mathrm{P}}=17.1 \mathrm{~Hz}, \mathrm{CH}_{3} \mathrm{CN}\right), 23.07(\mathrm{dd}, J=22.7,15.9 \mathrm{~Hz}, \mathrm{CH}), 20.72-20.41(\mathrm{~m}, \mathrm{CH})$, $20.29\left(\mathrm{~d}, J=7.6 \mathrm{~Hz}, \mathrm{CH}_{2}\right), 19.79\left(\mathrm{~d}, J=8.3 \mathrm{~Hz}, \mathrm{CH}_{2}\right), 19.15\left(\mathrm{~s}, \mathrm{CH}_{3}\right), 18.26\left(\mathrm{~s}, \mathrm{CH}_{3}\right) ;{ }^{11} \mathrm{~B}\left\{{ }^{1} \mathrm{H}\right\}$ $\operatorname{NMR}\left(128 \mathrm{MHz}, \mathrm{THF}-d_{8}\right) \delta-9.72\left(\mathrm{~s}, \mathrm{CN} B \mathrm{Ph}_{3}\right)$.

Anal. Calcd for $\mathrm{C}_{34} \mathrm{H}_{50} \mathrm{BNP}_{2} \mathrm{Pd}$ : C, 62.64; H, 7.73; N, 2.15. Found: C, 62.44; H, 7.70; N, 1.77 . Solid IR (ATR, $\left.\mathrm{cm}^{-1}\right): 1792\left(v_{\mathrm{CN}}\right)$ 


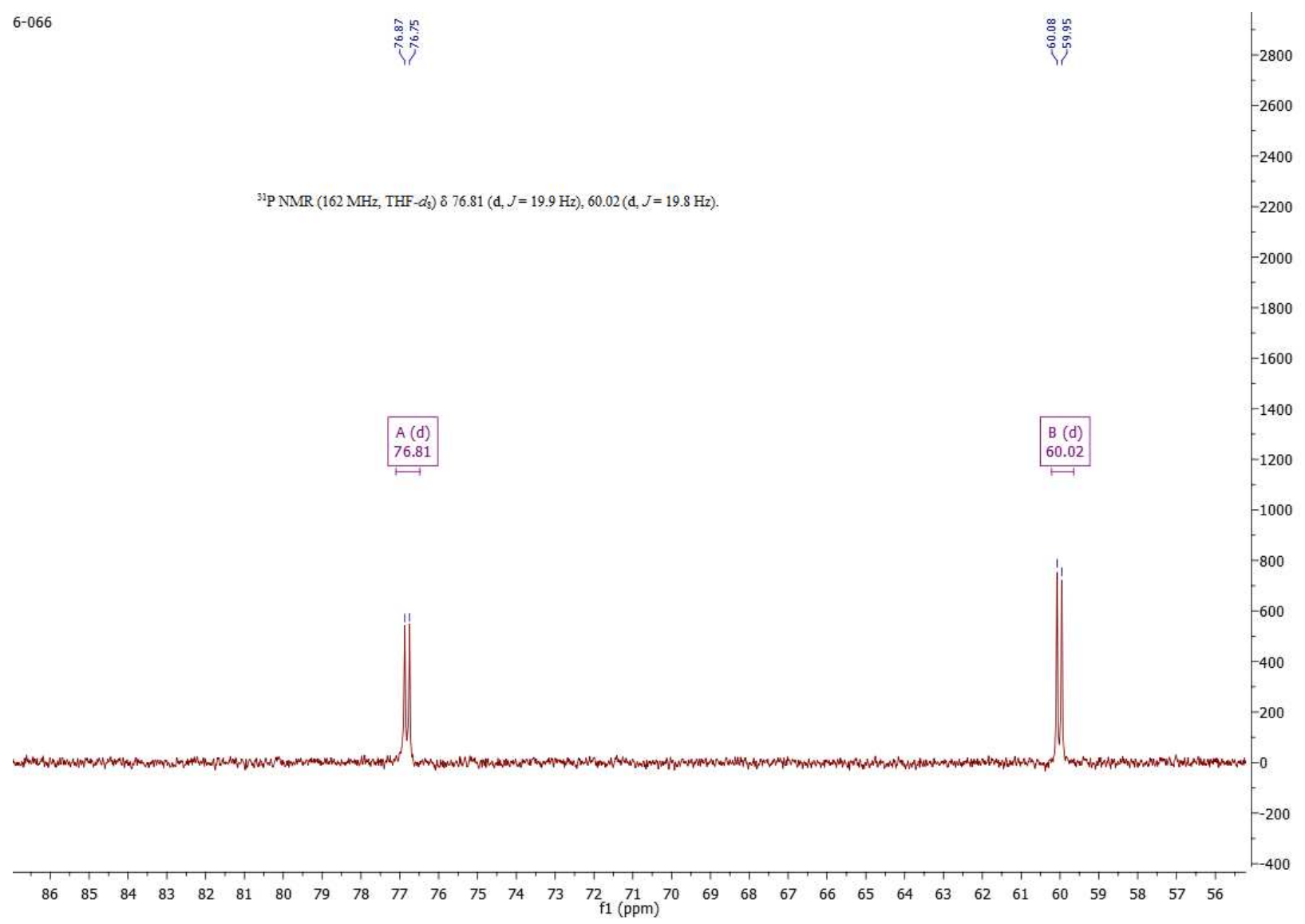

Figure S6: ${ }^{31 \mathrm{P}}\left\{{ }^{1} \mathrm{H}\right\}$ NMR of $\mathbf{2 a}$ ' in THF- $d_{8}$ 


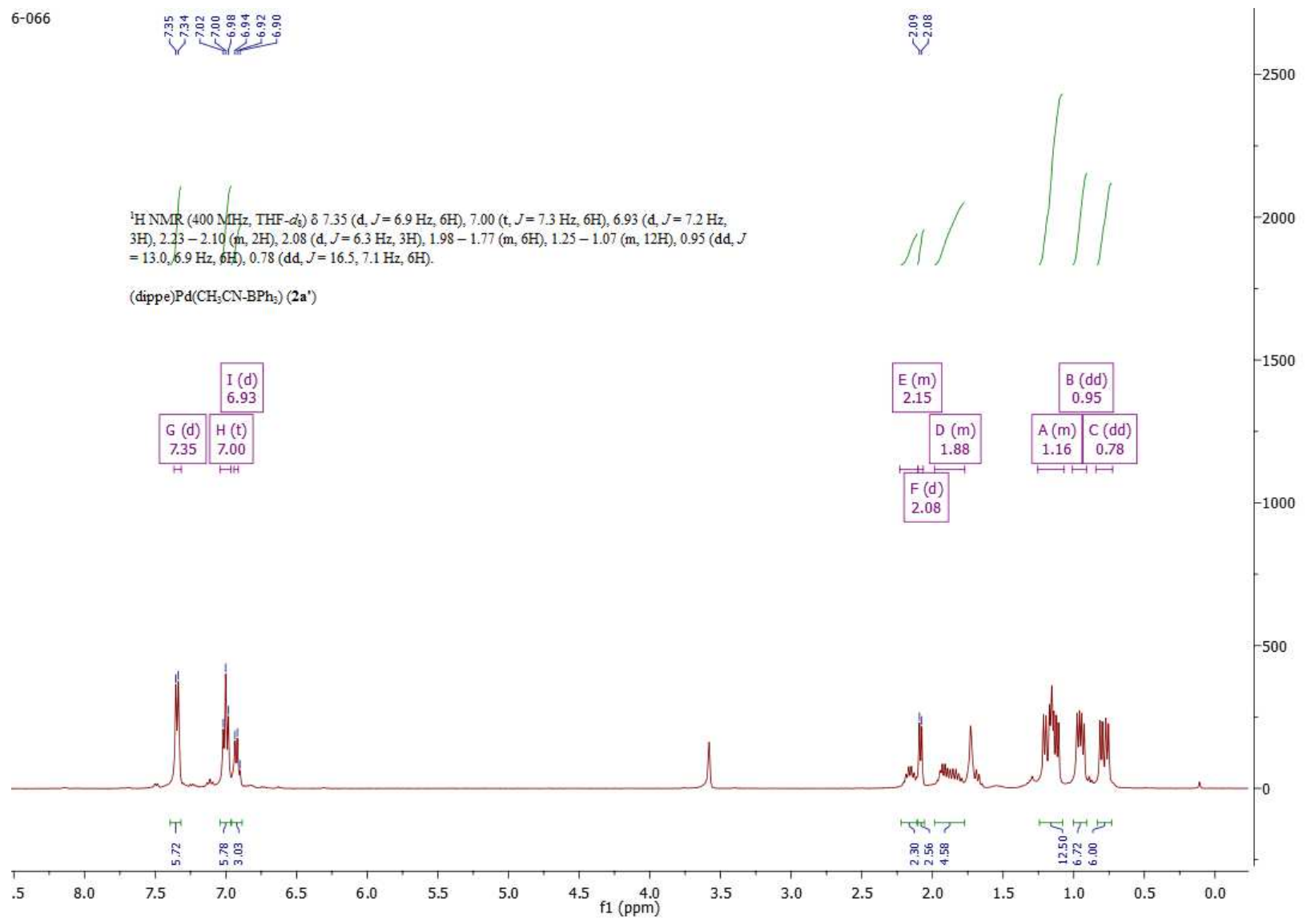

Figure S7: ${ }^{1} \mathrm{H}$ NMR of $\mathbf{2 a}$ ' in THF- $d_{8}$ 


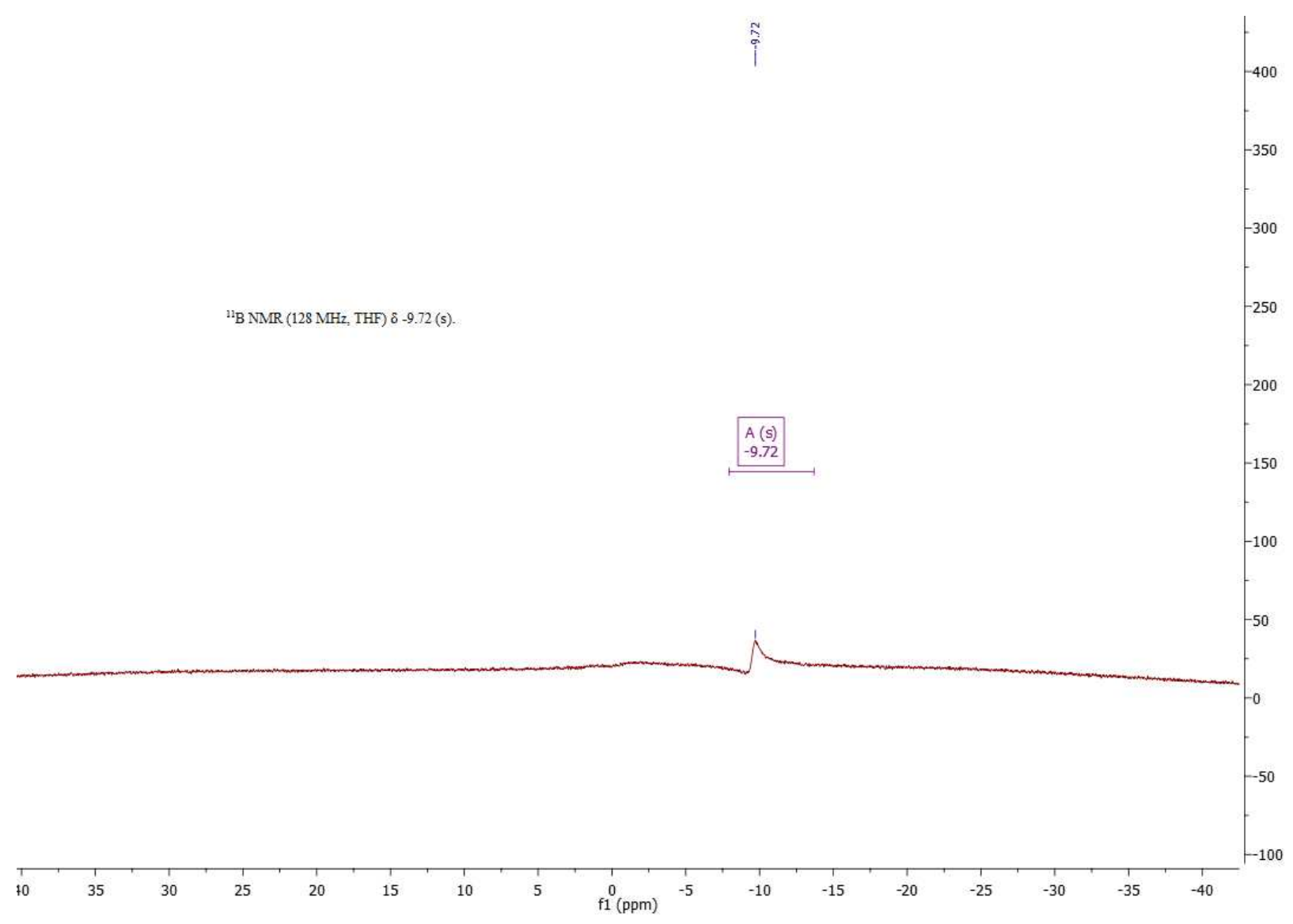

Figure S8: ${ }^{11} \mathrm{~B}\left\{{ }^{1} \mathrm{H}\right\}$ NMR of $\mathbf{2 a}$ ' in THF- $d_{8}$ 


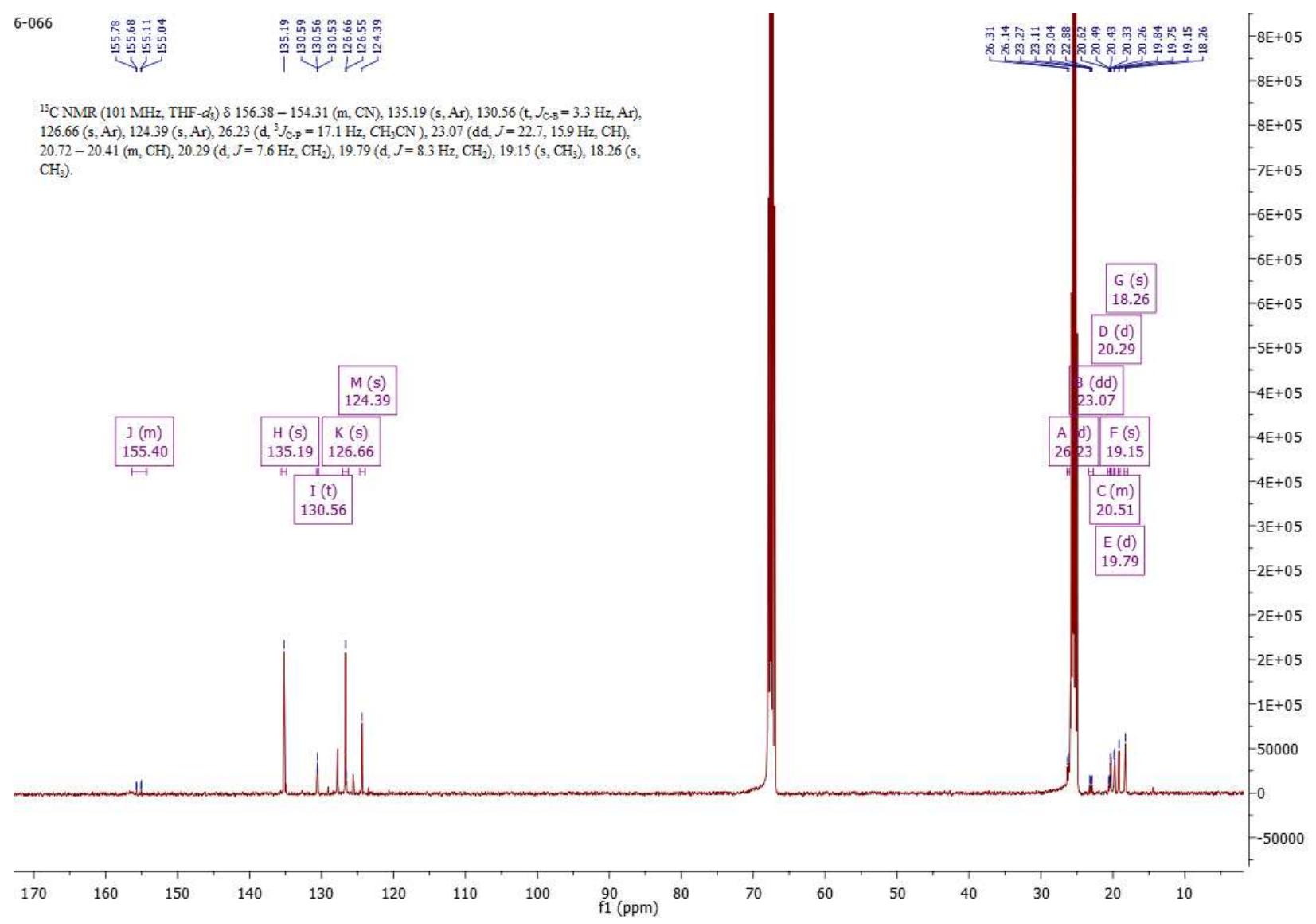

Figure S9: ${ }^{13} \mathrm{C}\left\{{ }^{1} \mathrm{H}\right\}$ NMR of $\mathbf{2 a}{ }^{\prime}$ in THF- $d_{8}$ 


\section{Preparation of complex $3 b($ dippe $) P d(P h)\left(C N-B E t_{3}\right)$}

Under a nitrogen atmosphere, $\mathrm{KHBEt}_{3}(0.9 \mathrm{~mL}$ of $1 \mathrm{M}$ solution in THF, $0.9096 \mathrm{mmol})$ was added to a stirred suspension of [(dippe) $\left.\mathrm{PdCl}_{2}\right](200 \mathrm{mg}, 0.4548 \mathrm{mmol})$ in $3.0 \mathrm{~mL}$ of toluene. The resulting intense dark red solution was then filtered through Celite before adding $0.5 \mathrm{~mL}$ of benzonitrile to the filtrate. After stirring for $10 \mathrm{~min}$, the light red solution was dried on vacuo to give a light red oil. The oil was dissolved in $0.1 \mathrm{~mL}$ of THF and $10 \mathrm{~mL}$ of pentane to wash of excess PhCN. This process was repeated three times until a pale yellow solid crushed out in solution. The pale yellow solid was dried in vacuo to yield 2a' (166 mg, 64 \%)

${ }^{1} \mathrm{H}$ NMR (400 MHz, THF- $\left.d_{8}\right) \delta 7.30(\mathrm{t}, J=7.0 \mathrm{~Hz}, 2 \mathrm{H}), 6.95(\mathrm{t}, J=6.8 \mathrm{~Hz}, 2 \mathrm{H}), 6.80(\mathrm{t}, J=7.1$ $\mathrm{Hz}, 1 \mathrm{H}), 2.43-2.32(\mathrm{~m}, 2 \mathrm{H}), 2.24-2.11(\mathrm{~m}, 2 \mathrm{H}), 2.10-1.80(\mathrm{~m}, 4 \mathrm{H}), 1.38(\mathrm{dd}, J=16.3,7.1$ $\mathrm{Hz}, 6 \mathrm{H}), 1.24(\mathrm{dd}, J=14.0,7.0 \mathrm{~Hz}, 6 \mathrm{H}), 1.14(\mathrm{dd}, J=14.6,6.9 \mathrm{~Hz}, 6 \mathrm{H}), 0.91(\mathrm{dd}, J=16.2,7.2$ $\mathrm{Hz}, 6 \mathrm{H}), 0.79-0.55(\mathrm{~m}, J=21.6,14.7,7.3 \mathrm{~Hz}, 9 \mathrm{H}), 0.15--0.15(\mathrm{~m}, 6 \mathrm{H}) ;{ }^{31} \mathrm{P}\left\{{ }^{1} \mathrm{H}\right\} \mathrm{NMR}(162$ $\left.\mathrm{MHz}, \mathrm{THF}-d_{8}\right) \delta 76.09\left(\mathrm{~d},{ }^{2} J_{\mathrm{P}-\mathrm{P}}=18.4 \mathrm{~Hz}\right), 70.43\left(\mathrm{~d},{ }^{2} J_{\mathrm{P}-\mathrm{P}}=18.3 \mathrm{~Hz}\right) ;{ }^{13} \mathrm{C} \mathrm{NMR}(101 \mathrm{MHz}$, THF-d $\left.d_{8}\right) \delta 138.87(\mathrm{~s}, \mathrm{CN}), 132.96(\mathrm{~s}, \mathrm{Ar}), 130.14(\mathrm{~s}, \mathrm{Ar}), 127.68$ (d, J=7.6 Hz, Ar), 123.60 (s, Ar), $23.94-23.11(\mathrm{~m}, \mathrm{CH}), 21.14-20.51(\mathrm{~m}, \mathrm{CH}), 20.18\left(\mathrm{~d}, J=4.5 \mathrm{~Hz}, \mathrm{CH}_{2}\right), 19.14(\mathrm{~d}, J=2.7$ $\left.\mathrm{Hz}, \mathrm{CH}_{2}\right), 19.00\left(\mathrm{~s}, \mathrm{CH}_{3}\right), 17.95\left(\mathrm{~s}, \mathrm{CH}_{3}\right), 11.58\left(\mathrm{~s}, \mathrm{CH}_{2}\right), 10.15\left(\mathrm{~s}, \mathrm{CH}_{3}\right) ;{ }^{11} \mathrm{~B}\left\{{ }^{1} \mathrm{H}\right\} \mathrm{NMR}(128$ $\left.\mathrm{MHz}, \mathrm{THF}-d_{8}\right) \delta-17.35\left(\mathrm{~s}, \mathrm{CN} B \mathrm{Et}_{3}\right)$.

Solid IR (ATR, $\left.\mathrm{cm}^{-1}\right): 1720\left(v_{\mathrm{CN}}\right)$ Anal. Calcd for $\mathrm{C}_{27} \mathrm{H}_{52} \mathrm{BNP}_{2} \mathrm{Pd}: \mathrm{C}, 56.90 ; \mathrm{H}, 9.20 ; \mathrm{N}, 2.46$; Found: C, 53.51; H, 8.72; N, 2.90 
${ }^{1} \mathrm{H}$ NMR $\left(400 \mathrm{MHz}\right.$, THF- $\left.d_{\mathrm{s}}\right) \delta 7.30(\mathrm{t}, J=7.0 \mathrm{~Hz}, 2 \mathrm{H}), 6.95(\mathrm{t}, J=6.8 \mathrm{~Hz}, 2 \mathrm{H}), 6.80(\mathrm{t}, J=7.1 \mathrm{~Hz}, 1 \mathrm{H})$, $2.43-2.32(\mathrm{~m}, 2 \mathrm{H}), 2.24-2.11(\mathrm{~m}, 2 \mathrm{H}), 2.10-1.80(\mathrm{~m}, 4 \mathrm{H}), 1.38(\mathrm{dd}, J=16.3,7.1 \mathrm{~Hz}, 6 \mathrm{H}), 1.24(\mathrm{dd}, J$ $=14.0,7.0 \mathrm{~Hz}, 6 \mathrm{H}), 1.14(\mathrm{dd}, J=14.6,6.9 \mathrm{~Hz}, 6 \mathrm{H}), 0.91(\mathrm{dd}, J=16.2,7.2 \mathrm{~Hz}, 6 \mathrm{H}), 0.79-0.55(\mathrm{~m}, J=$ $21.6,14.7,7.3 \mathrm{~Hz}, 9 \mathrm{H}), 0.15--0.15(\mathrm{~m}, 6 \mathrm{H})$

(dippe) $\mathrm{Pd}(\mathrm{Ph})\left(\mathrm{CN}-\mathrm{BEt}_{3}\right)(3 \mathrm{~b})$
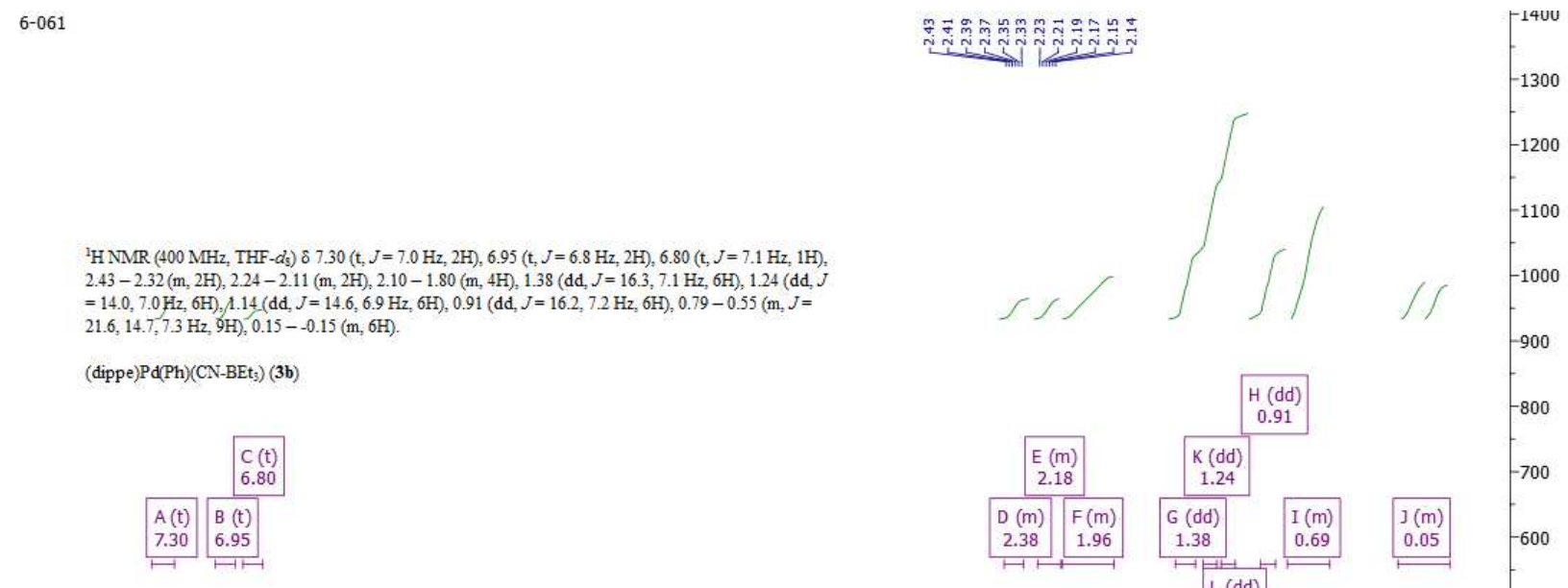

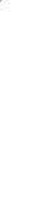
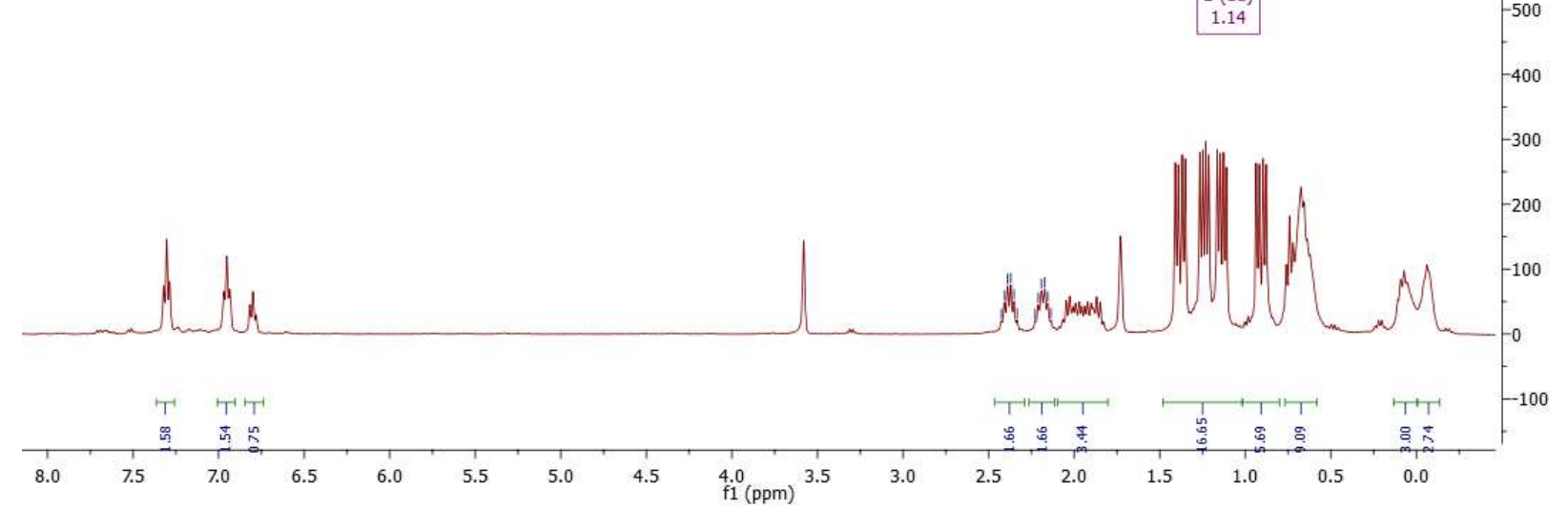

Figure S10: ${ }^{1} \mathrm{H}$ NMR of $\mathbf{3 b}$ in THF- $d_{8}$ 


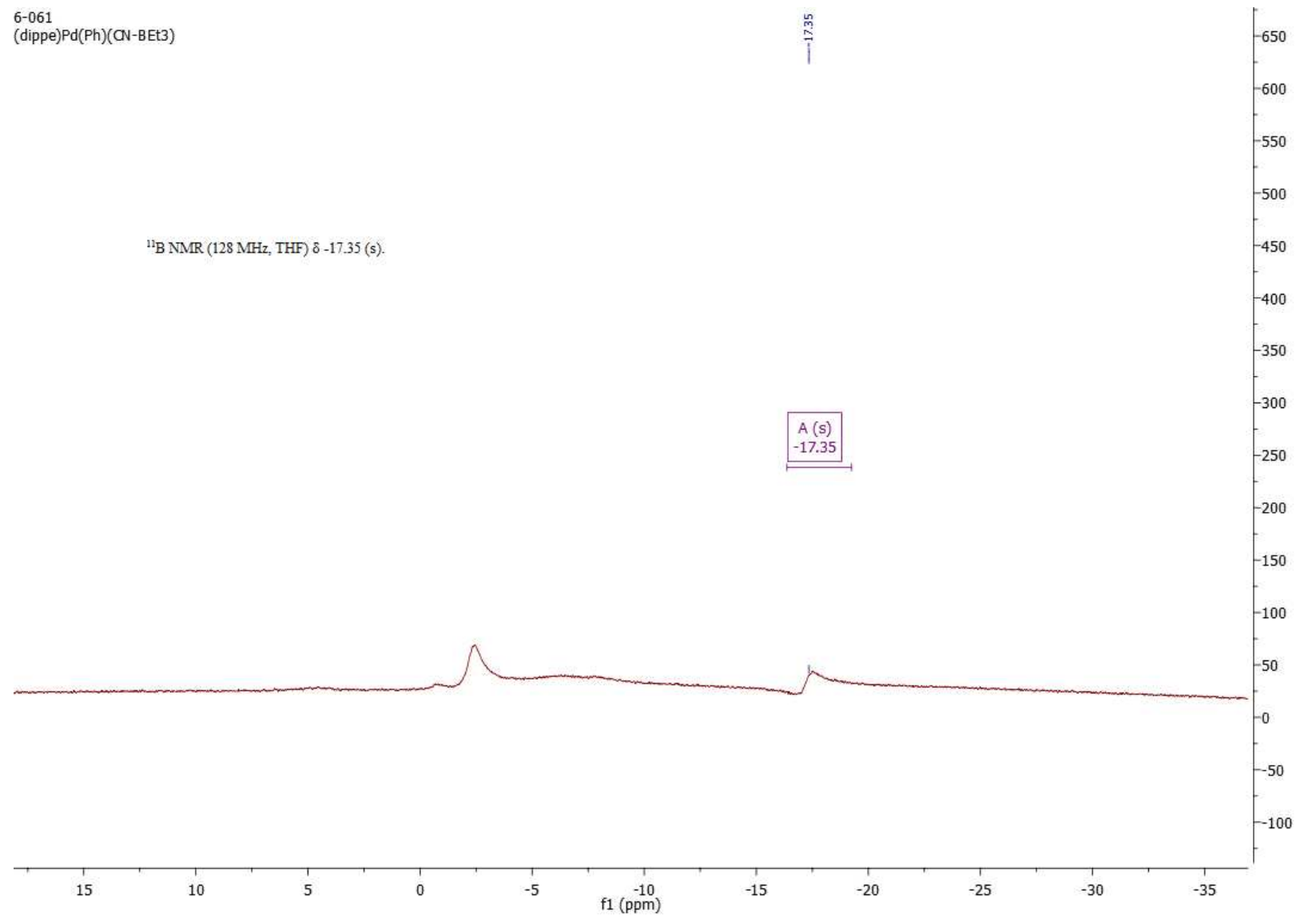

Figure S11: ${ }^{11} \mathrm{~B}\left\{{ }^{1} \mathrm{H}\right\}$ NMR of $\mathbf{3 b}$ in THF- $d_{8}$ 


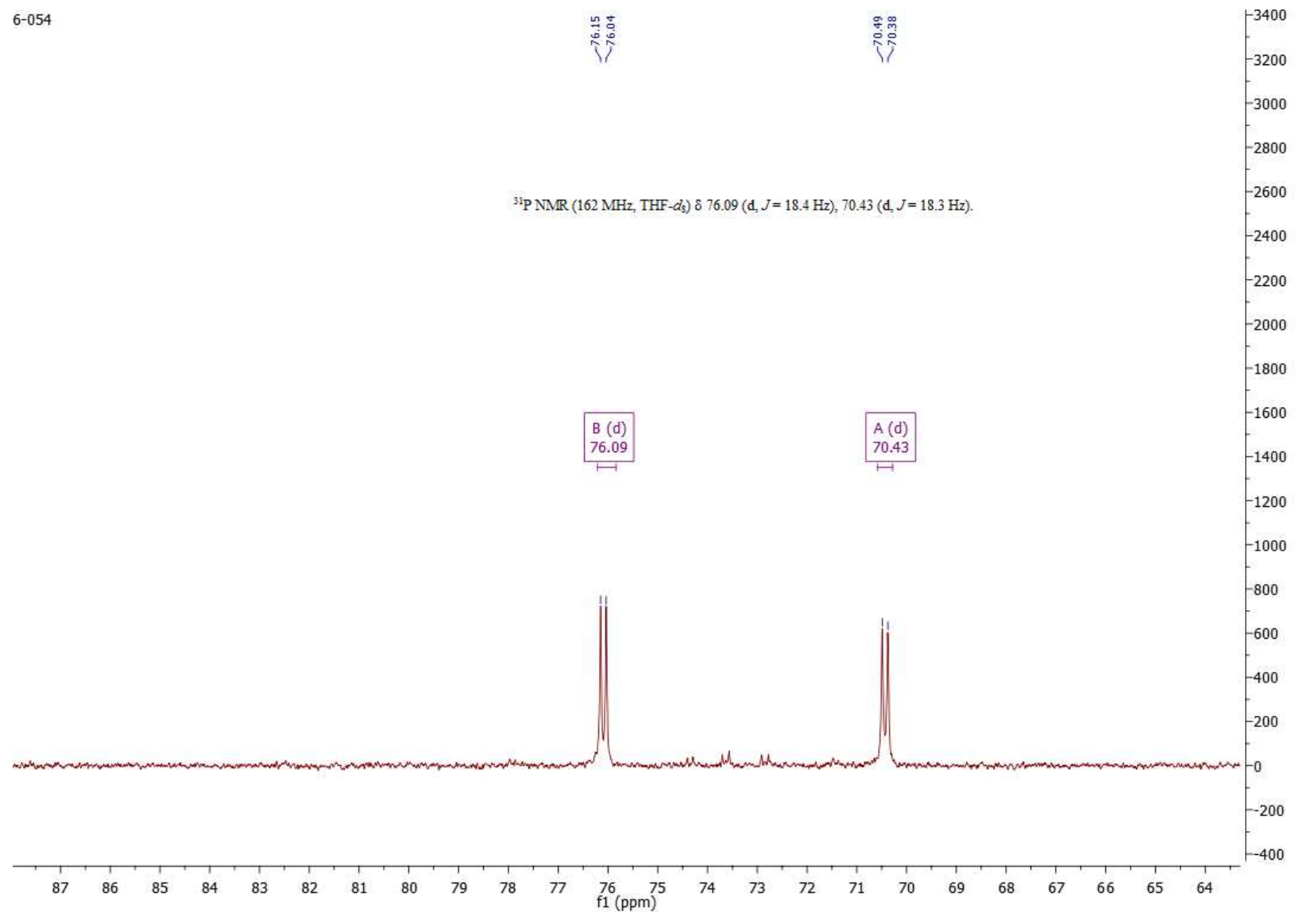

Figure S12: ${ }^{31} \mathrm{P}\left\{{ }^{1} \mathrm{H}\right\}$ NMR of $\mathbf{3 b}$ in THF- $d_{8}$ 


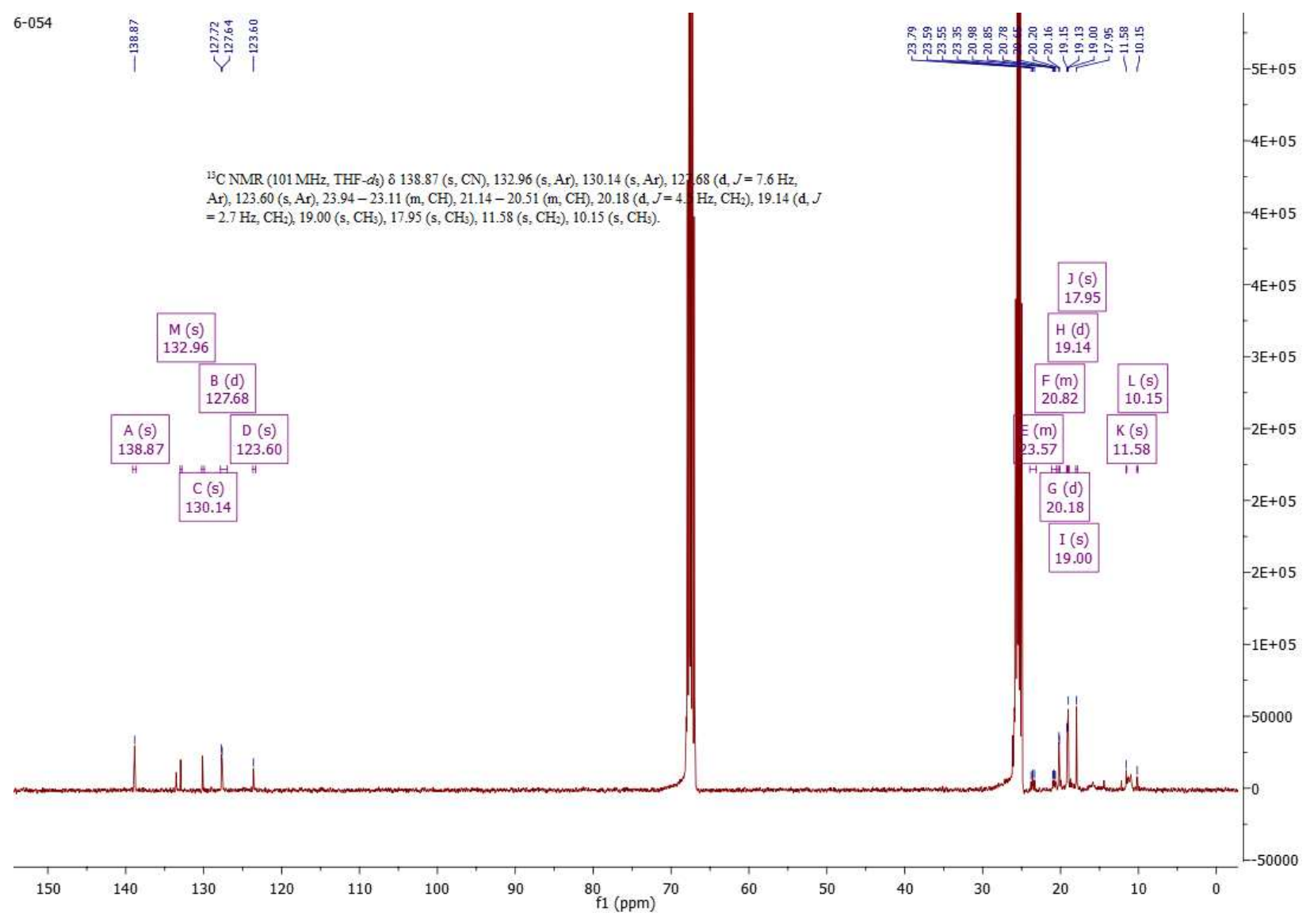

Figure S13: ${ }^{13} \mathrm{C}\left\{{ }^{1} \mathrm{H}\right\}$ NMR of $\mathbf{3 b}$ in THF- $d_{8}$ 


\section{Preparation of complex 3a' (dippe) $\mathrm{Pd}\left(\mathrm{CH}_{3}\right)\left(\mathrm{CN}-\mathrm{BPh}_{3}\right)+($ dippe $) \mathrm{Pd}-\mathrm{H}$ products}

Three $J$. Young NMR tubes samples were prepared each contained (dippe) $\operatorname{Pd}\left(\eta^{2}-\mathrm{C}, \mathrm{N}-\right.$ $\left.\mathrm{CH}_{3} \mathrm{CNBPh}_{3}\right)(10 \mathrm{mg}, 0.015 \mathrm{mmol})$ dissolved in $0.5 \mathrm{~mL}$ in THF- $d_{8}$. One was heated at $100{ }^{\circ} \mathrm{C}$, another at $65{ }^{\circ} \mathrm{C}$ and the other one was photolyzed at room temperature. The results from the three separate experiments are shown below.

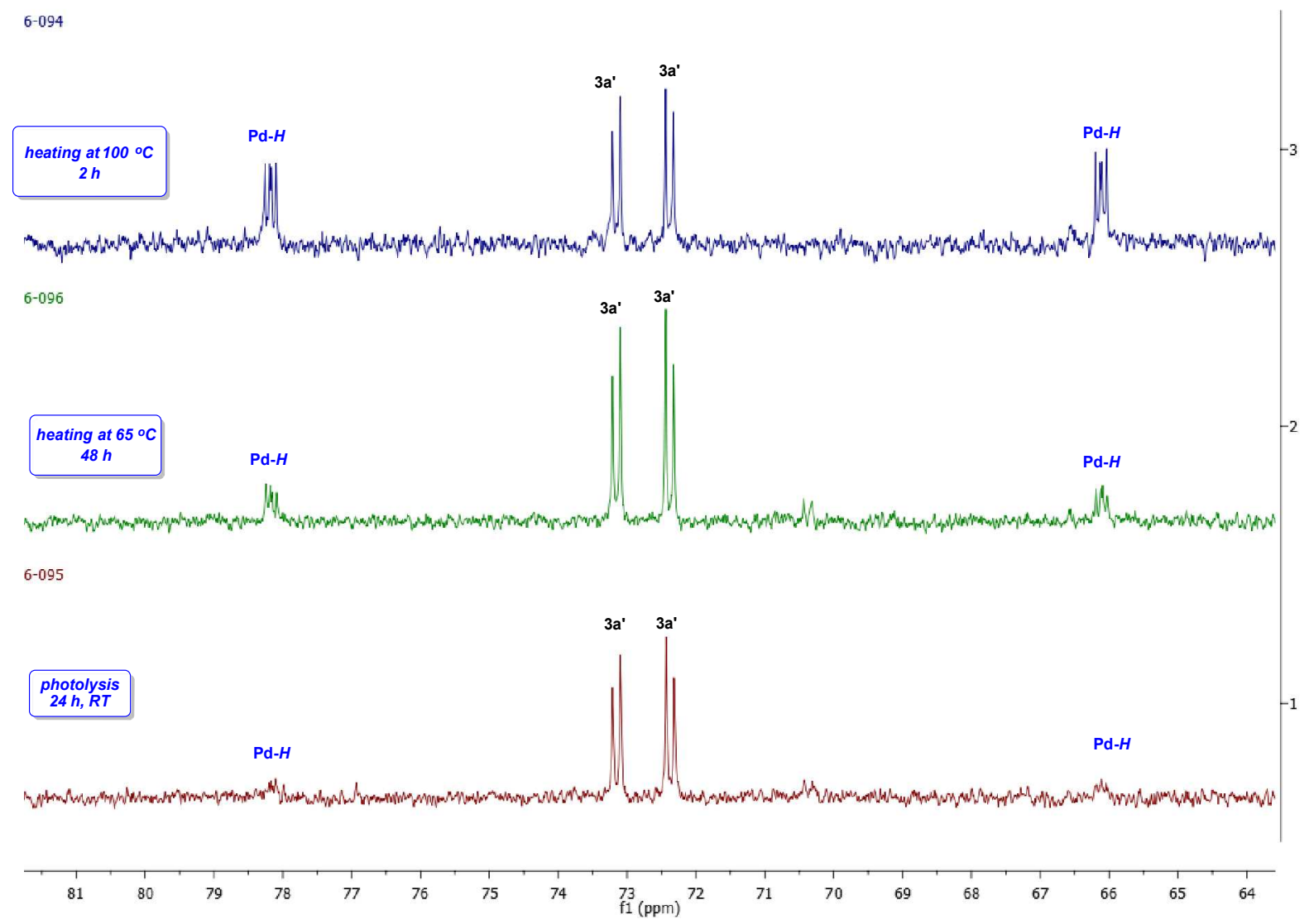

Figure S14. ${ }^{31} \mathrm{P}\left\{{ }^{1} \mathrm{H}\right\}$ NMR of reactions of complex $\mathbf{2 a}$ ' at different reaction conditions yielding 3a', (dippe) $\mathrm{Pd}\left(\mathrm{CH}_{3}\right)\left(\mathrm{CNBPh}_{3}\right)$ and unidentified (dippe) $\mathrm{Pd}-\boldsymbol{H}$ products. 


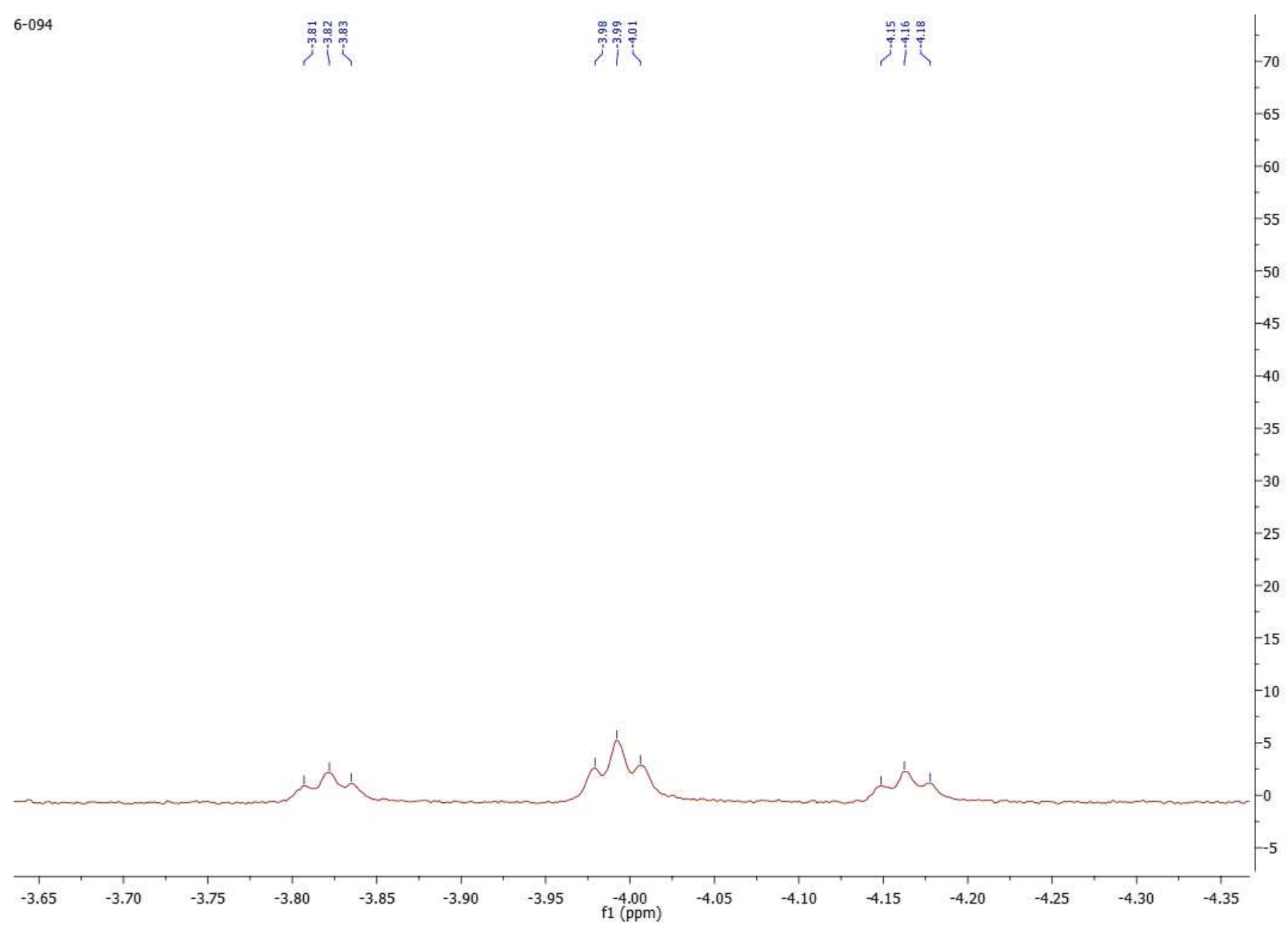

Figure S15. Hydride region for reaction at 100 and $65{ }^{\circ} \mathrm{C}$ showing (dippe) $P d-H$ product/s.

Photolysis experiment shows minimal hydride formation. 


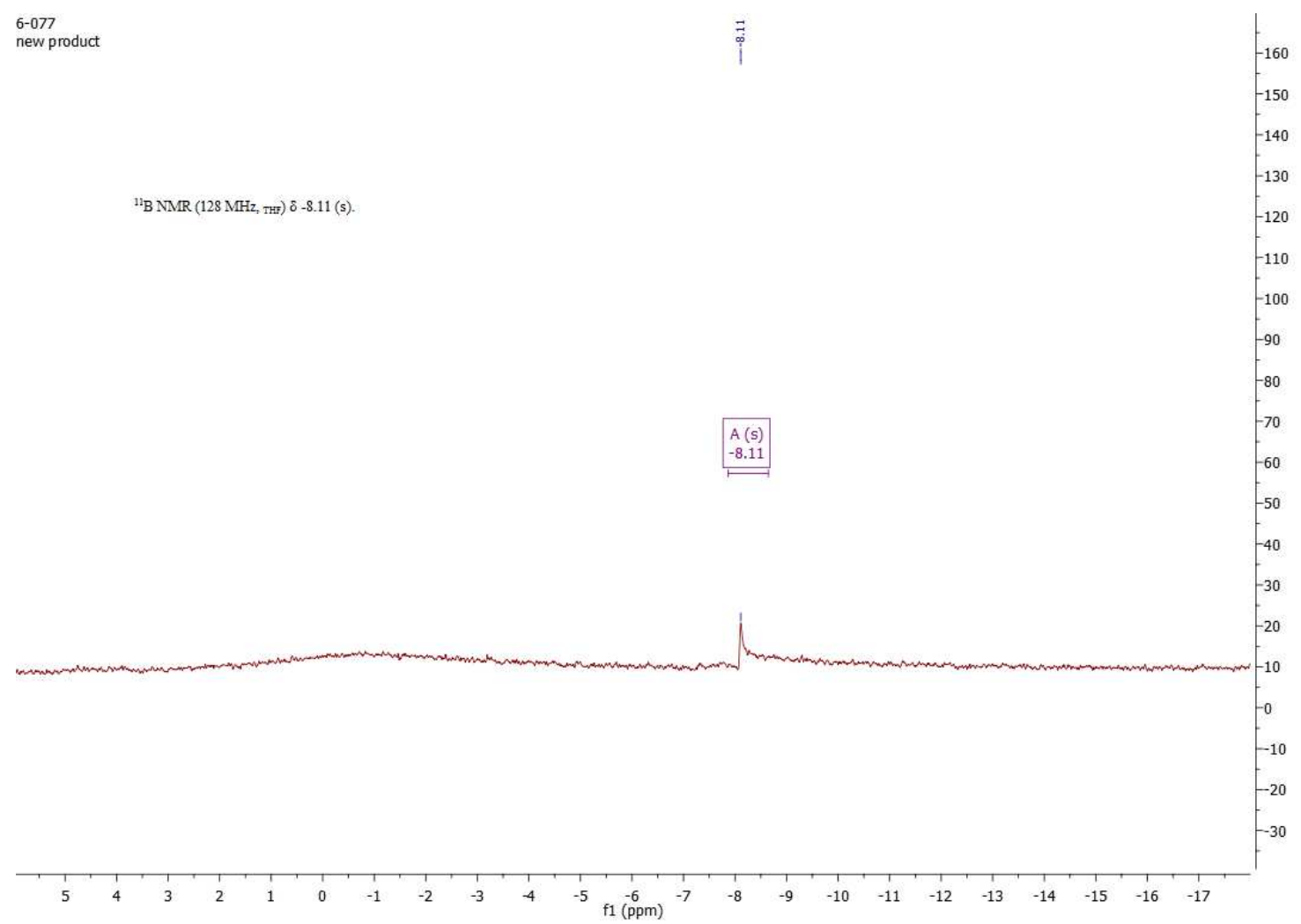

Figure S16: ${ }^{11} \mathrm{~B}\left\{{ }^{1} \mathrm{H}\right\}$ NMR of 3a' in THF- $d_{8}$ 


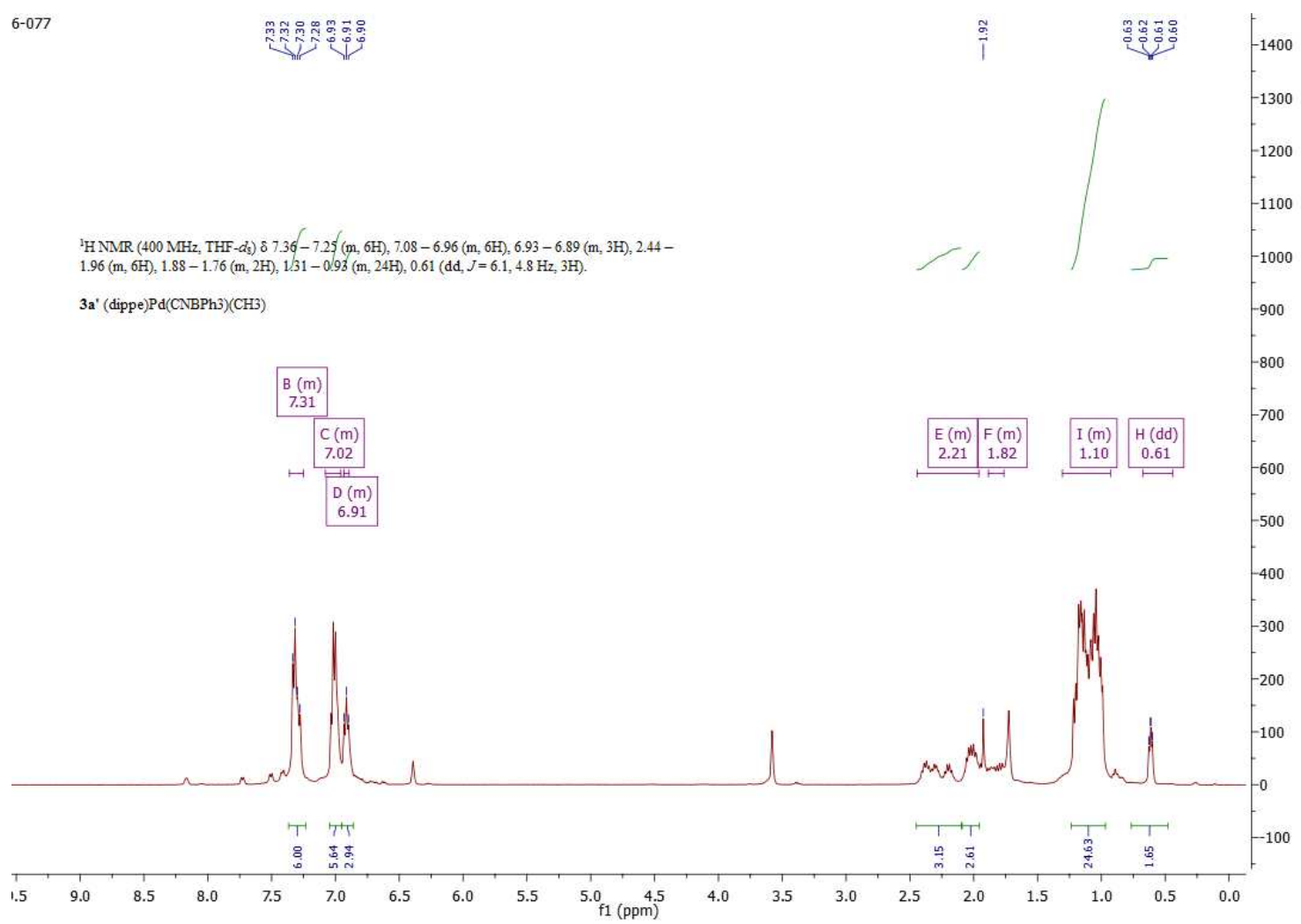

Figure S17: ${ }^{1} \mathrm{H}$ NMR of $\mathbf{3 a}$ ' in THF- $d_{8}$.

${ }^{1} \mathrm{H}$ NMR for 3a' (400 MHz, THF- $\left.d_{8}\right) \delta 7.36-7.25(\mathrm{~m}, 6 \mathrm{H}), 7.08-6.96(\mathrm{~m}, 6 \mathrm{H}), 6.93-6.89(\mathrm{~m}$, $3 \mathrm{H}), 2.44-1.96(\mathrm{~m}, 6 \mathrm{H}), 1.88-1.76(\mathrm{~m}, 2 \mathrm{H}), 1.31-0.93(\mathrm{~m}, 24 \mathrm{H}), 0.61\left(\mathrm{dd},{ }^{3} J_{\mathrm{H}-\mathrm{P}}=6.1,4.8 \mathrm{~Hz}\right.$, $3 \mathrm{H}) .{ }^{31} \mathrm{P}\left\{{ }^{1} \mathrm{H}\right\}$ NMR $\left(162 \mathrm{MHz}, \mathrm{THF}, \mathrm{THF}-d_{8}\right) \delta 73.15(\mathrm{~d}, J=18.7 \mathrm{~Hz}), 72.40(\mathrm{~d}, J=18.7 \mathrm{~Hz})$, 
Preparation of complex 3a (dippe) $\mathrm{Pd}\left(\mathrm{CH}_{3}\right)\left(\mathrm{CN}-\mathrm{BEt}_{3}\right)$ by photolysis

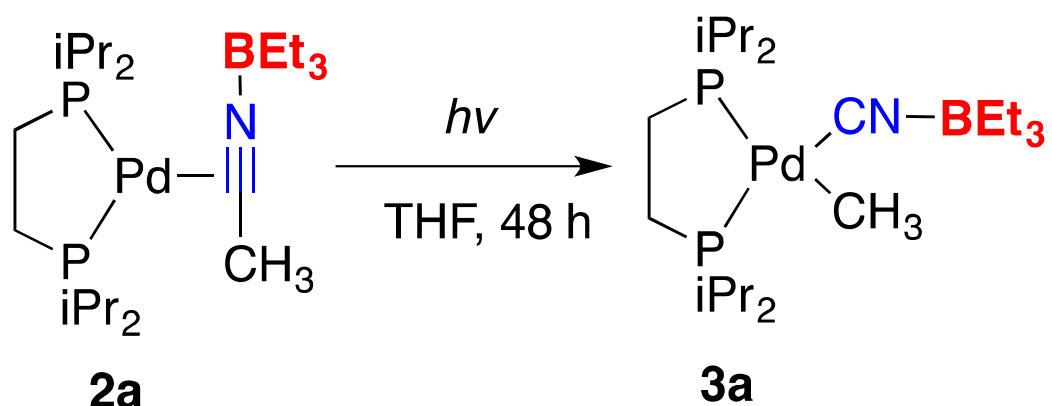

Complex 2a, (dippe) $\mathrm{Pd}\left(\mathrm{CH}_{3} \mathrm{CN}-\mathrm{BEt}_{3}\right)$, generated in situ from the procedure shown on page $\mathrm{C} 3$ is exposed to photolysis $(\lambda>300 \mathrm{~nm})$ for $48 \mathrm{~h}$ for complete conversion. C-CN activation of $2 \mathrm{a}$ to give (dippe) $\mathrm{Pd}\left(\mathrm{CH}_{3}\right)\left(\mathrm{CN}-\mathrm{BEt}_{3}\right) \mathbf{3 a}$ is achieved. In addition to formation of $\mathbf{3 a}$, another unidentified phosphorous product is obtained ( $\delta$ 63, s) as shown by the ${ }^{31} \mathrm{P}\left\{{ }^{1} \mathrm{H}\right\}$ spectra below (Figure S17). Due to challenges isolating complex 3a due to low stability and decomposition issues, only the ${ }^{31} \mathrm{P}\left\{{ }^{1} \mathrm{H}\right\}$ spectrum is provided. The coupling constant for $\mathbf{3 a}$ of ${ }^{2} J_{\mathrm{P}-\mathrm{P}}=18.4 \mathrm{~Hz}$ are consistent with asymmetric (dippe)Pd(II) species. ${ }^{2}$ 


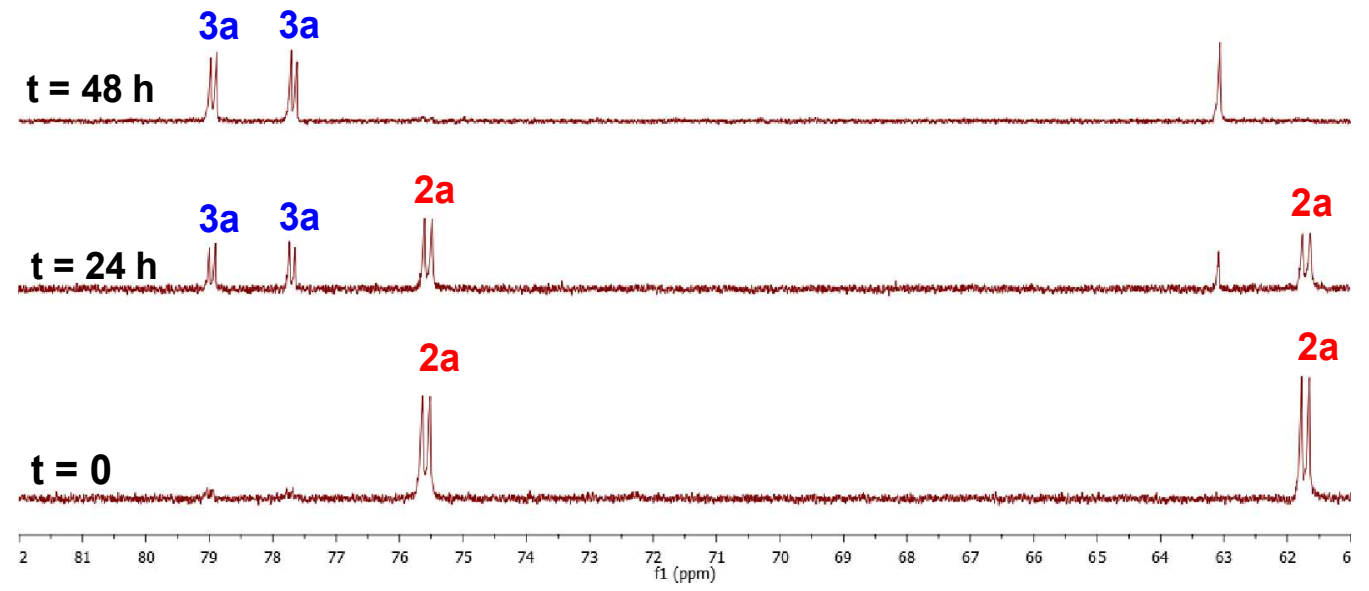

Figure S18. ${ }^{31} \mathrm{P}\left\{{ }^{1} \mathrm{H}\right\}$ NMR spectra for photolysis of 2a in THF solution over 48h. 2a: (dippe) $\mathrm{Pd}\left(\eta^{2}-\mathrm{C}, \mathrm{N}-\mathrm{CH}_{3} \mathrm{CN}-\mathrm{BEt}_{3}\right),\left({ }^{2} J_{\mathrm{P}-\mathrm{P}}=25.0 \mathrm{~Hz}\right)$ 3a: $($ dippe $) \mathrm{Pd}\left(\mathrm{CH}_{3}\right)\left(\mathrm{CN}_{-} \mathrm{BEt}_{3}\right),\left({ }^{2} J_{\mathrm{P}-\mathrm{P}}=18.4\right.$ $\mathrm{Hz}$ ) and unidentified singlet resonance at $\delta 63$. 
REFERENCE NUMBER: jonlm07 (2a)

\title{
CRYSTAL STRUCTURE REPORT
}

\author{
$\mathrm{C}_{22} \mathrm{H}_{50}$ B N P 2 Pd \\ or \\ (dippe) $\mathrm{Pd}\left(\eta^{2}-\mathrm{MeC}=\mathrm{NBEt}_{3}\right)$
}

Report prepared for:

C. Torres, L. Munjanja, Prof. W. Jones

July 24,2013

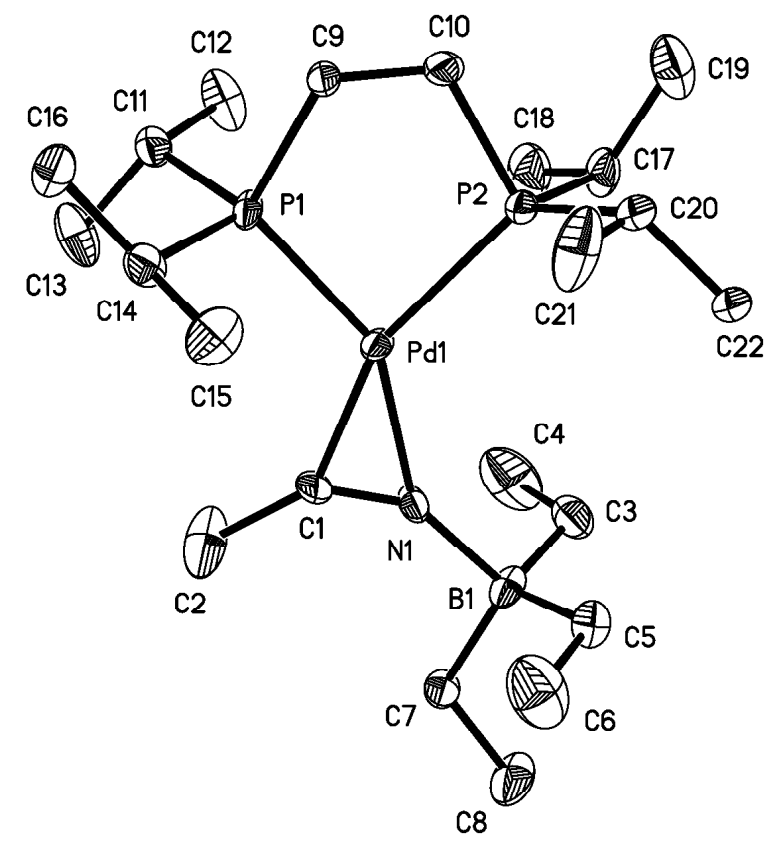

\footnotetext{
William W. Brennessel

X-ray Crystallographic Facility

Department of Chemistry, University of Rochester

120 Trustee Road

Rochester, NY 14627
} 


\section{Data collection}

A crystal $\left(0.24 \times 0.20 \times 0.10 \mathrm{~mm}^{3}\right)$ was placed onto the tip of a $0.1 \mathrm{~mm}$ diameter glass capillary tube or fiber and mounted on a Bruker SMART APEX II CCD platform diffractometer for a data collection at $100.0(5) \mathrm{K}^{1}$ A preliminary set of cell constants and an orientation matrix were calculated from reflections harvested from three orthogonal wedges of reciprocal space. The full data collection was carried out using MoK $\alpha$ radiation (graphite monochromator) with a frame time of 60 seconds and a detector distance of $4.01 \mathrm{~cm}$. A randomly oriented region of reciprocal space was surveyed: four major sections of frames were collected with $0.50^{\circ}$ steps in $\omega$ at four different $\phi$ settings and a detector position of $-38^{\circ}$ in $2 \theta$. The intensity data were corrected for absorption. $^{2}$ Final cell constants were calculated from the xyz centroids of 3957 strong reflections from the actual data collection after integration. ${ }^{3}$ See Table S1 for additional crystal and refinement information.

Structure solution and refinement

The structure was solved using SIR20114 and refined using SHELXL-2013.5 The space group $P n a 2_{1}$ was determined based on systematic absences. Intensity statistics suggested space group Pnma, but it was soon discovered that the high $E^{2}-1$ value was due to severe hypersymmetry (see below). A direct-methods solution was calculated which provided most non-hydrogen atoms from the E-map. Full-matrix least squares / difference Fourier cycles were performed which located the remaining non-hydrogen atoms. All non-hydrogen atoms were refined with anisotropic displacement parameters. All hydrogen atoms were placed in ideal positions and refined as riding atoms with relative isotropic displacement parameters.

The two independent molecules are closely related by a non-crystallographic center of symmetry, a symmetry equivalent of which occurs at $x y z$ coordinate $0.124,-0.008,0.725$. According to previous studies, ${ }^{6} x=1 / 8, y=0$ is a common location for such a center in space group Pna $2_{1}$. This form of hypersymmetry causes severe correlations among the atomic coordinates related by the center. Because of this, corresponding bond lengths between the two molecules were restrained to be similar and corresponding thermal parameters were constrained to be equivalent.

The largest peaks of 7.14 and 6.14 electrons per $\AA^{3}$ are located 0.57 and $0.59 \AA$ from atoms Pd1 and $\mathrm{Pd} 2$, respectively. The largest hole of -3.72 electrons per $\AA^{3}$ is located $0.48 \AA$ from atom $\mathrm{Pd} 1$ (and there is likely a corresponding hole near atom Pd2). It is not known to what these peaks and holes are due, and they have no chemical meaning relative to the defined molecules. 
The final full matrix least squares refinement converged to $R 1=0.1195\left(F^{2}, I>2 \sigma(I)\right)$ and $w R 2$ $=0.2601\left(F^{2}\right.$, all data).

Structure description

The structure is similar to the one suggested. As mentioned above, there are two molecules in the asymmetric unit in general positions that are related by a non-crysytallographic inversion center.

Unless noted otherwise all structural diagrams containing thermal displacement ellipsoids are drawn at the $50 \%$ probability level.

Data collection, structure solution, and structure refinement were conducted at the X-ray Crystallographic Facility, B51 Hutchison Hall, Department of Chemistry, University of Rochester. All publications arising from this report MUST either 1) include William W. Brennessel as a coauthor or 2) acknowledge William W. Brennessel and the X-ray Crystallographic Facility of the Department of Chemistry at the University of Rochester.

1 APEX2, version 2013.2-0; Bruker AXS: Madison, WI, 2013.

2 Sheldrick, G. M. SADABS, version 2012/1; University of Göttingen: Göttingen, Germany, 2012.

3 SAINT, version 8.27B; Bruker AXS: Madison, WI, 2013.

4 Burla, M. C.; Caliandro, R.; Camalli, M.; Carrozzini, B.; Cascarano, G. L.; Giacovazzo, C.; Mallamo, M.; Mazzone, A.; Polidori, G.; Spagna, R. SIR2011: a new package for crystal structure determination and refinement, version 1.0; Istituto di Cristallografia: Bari, Italy, 2012.

5 Sheldrick, G. M. SHELXL-2013/4 University of Göttingen: Göttingen, Germany, 2013.

6 Marsh, R. E.; Schomaker, V.; Herbstein, F. H. Acta. Cryst. 1998, B54, 921-924.

Some equations of interest:

$$
\begin{gathered}
R_{\text {int }}=\Sigma\left|F_{\mathrm{O}}^{2}-<F_{\mathrm{O}}^{2}>\right| / \Sigma\left|F_{\mathrm{o}}{ }^{2}\right| \\
R 1=\square\left\|F_{\mathrm{o}}|-| F_{\mathrm{c}}\right\| / \Sigma\left|F_{\mathrm{o}}\right| \\
w R 2=\left[\Sigma\left[w\left(F_{\mathrm{o}}^{2}-F_{\mathrm{c}}{ }^{2}\right)^{2}\right] / \Sigma\left[w\left(F_{\mathrm{o}}^{2}\right)^{2}\right]\right]^{1 / 2} \\
\text { where } w=1 /\left[\sigma^{2}\left(F_{\mathrm{o}}^{2}\right)+(a P)^{2}+b P\right] \text { and } \\
P=1 / 3 \max \left(0, F_{\mathrm{o}}^{2}\right)+2 / 3 F_{\mathrm{c}}^{2} \\
\text { GOF }=S=\left[\Sigma\left[w\left(F_{\mathrm{o}}^{2}-F_{\mathrm{c}}{ }^{2}\right)^{2}\right] /(m-n)\right]^{1 / 2}
\end{gathered}
$$


where $m=$ number of reflections and $n=$ number of parameters
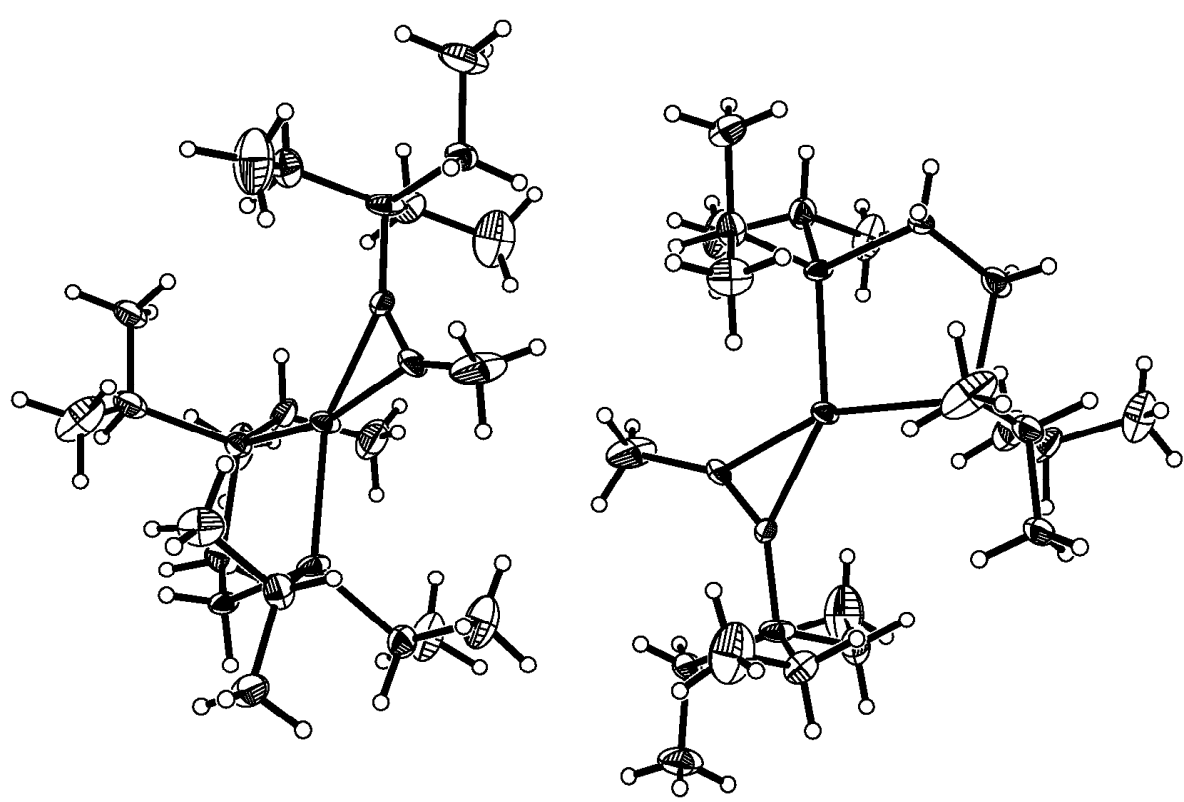


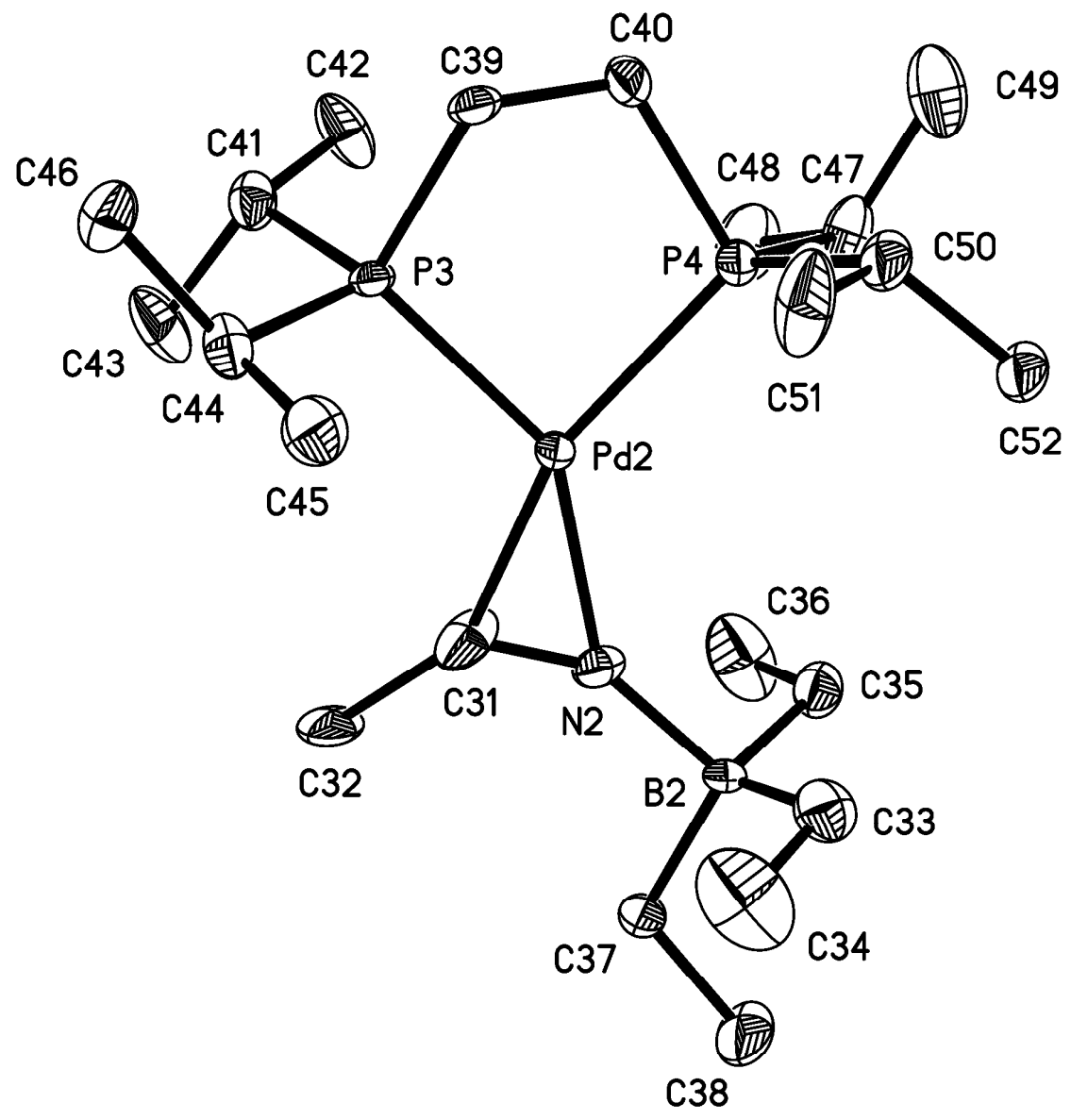


Table S1. Crystal data and structure refinement for jonlm07 (2a).

\begin{tabular}{|c|c|c|}
\hline Identification code & \multicolumn{2}{|l|}{ jonlm07 (2a) } \\
\hline Empirical formula & \multicolumn{2}{|l|}{ C22 H50 B N P2 Pd } \\
\hline Formula weight & \multicolumn{2}{|l|}{507.78} \\
\hline Temperature & \multicolumn{2}{|l|}{$100.0(5) \mathrm{K}$} \\
\hline Wavelength & \multicolumn{2}{|l|}{$0.71073 \AA$} \\
\hline Crystal system & \multicolumn{2}{|l|}{ orthorhombic } \\
\hline Space group & \multicolumn{2}{|l|}{$\operatorname{Pna}_{1}$} \\
\hline \multirow[t]{3}{*}{ Unit cell dimensions } & $a=28.6819(19) \AA$ & $\alpha=90^{\circ}$ \\
\hline & $b=9.0185(6) \AA$ & $\beta=90^{\circ}$ \\
\hline & $c=21.1144(14) \AA$ & $\gamma=90^{\circ}$ \\
\hline Volume & \multicolumn{2}{|l|}{$5461.6(6) \AA^{3}$} \\
\hline$Z$ & \multicolumn{2}{|l|}{8} \\
\hline Density (calculated) & \multicolumn{2}{|l|}{$1.235 \mathrm{Mg} / \mathrm{m}^{3}$} \\
\hline Absorption coefficient & \multicolumn{2}{|l|}{$0.804 \mathrm{~mm}^{-1}$} \\
\hline$F(000)$ & \multicolumn{2}{|l|}{2160} \\
\hline Crystal color, morphology & \multicolumn{2}{|l|}{ yellow-orange, plate } \\
\hline Crystal size & \multicolumn{2}{|c|}{$0.24 \times 0.20 \times 0.10 \mathrm{~mm}^{3}$} \\
\hline Theta range for data collection & \multicolumn{2}{|l|}{1.420 to $34.984^{\circ}$} \\
\hline Index ranges & \multicolumn{2}{|c|}{$-46 \leq h \leq 46,-14 \leq k \leq 14,-34 \leq l \leq 34$} \\
\hline Reflections collected & \multicolumn{2}{|l|}{112832} \\
\hline Independent reflections & \multicolumn{2}{|c|}{$24006[R($ int $)=0.0936]$} \\
\hline Observed reflections & \multicolumn{2}{|l|}{16071} \\
\hline Completeness to theta $=34.970^{\circ}$ & \multicolumn{2}{|l|}{$100.0 \%$} \\
\hline Absorption correction & \multicolumn{2}{|l|}{ Multi-scan } \\
\hline Max. and min. transmission & \multicolumn{2}{|l|}{0.7476 and 0.6490} \\
\hline Refinement method & \multicolumn{2}{|c|}{ Full-matrix least-squares on $F^{2}$} \\
\hline Data / restraints / parameters & \multicolumn{2}{|l|}{$24006 / 192$ / 342} \\
\hline Goodness-of-fit on $F^{2}$ & \multicolumn{2}{|l|}{1.085} \\
\hline Final $R$ indices $[I>2 \operatorname{sigma}(I)]$ & \multicolumn{2}{|c|}{$R 1=0.1195, w R 2=0.2370$} \\
\hline$R$ indices (all data) & \multicolumn{2}{|c|}{$R 1=0.1627, w R 2=0.2601$} \\
\hline Absolute structure parameter & \multicolumn{2}{|l|}{$0.48(11)$} \\
\hline Largest diff. peak and hole & \multicolumn{2}{|c|}{7.141 and -3.725 e..$\AA^{-3}$} \\
\hline
\end{tabular}


Table S2. Atomic coordinates $\left(\mathrm{x} 10^{4}\right)$ and equivalent isotropic displacement parameters $\left(\AA^{2} \mathrm{X}\right.$ $10^{3}$ ) for jonlm07 (2a). $U_{\text {eq }}$ is defined as one third of the trace of the orthogonalized $U_{i j}$ tensor.

\begin{tabular}{|c|c|c|c|c|}
\hline - & $\mathrm{x}$ & $\mathrm{y}$ & $\mathrm{z}$ & $\mathrm{U}_{\mathrm{eq}}$ \\
\hline \multicolumn{5}{|l|}{-} \\
\hline $\mathrm{Pd} 1$ & $2264(1)$ & $2448(1)$ & $6634(1)$ & $19(1)$ \\
\hline P1 & $2002(1)$ & $2846(5)$ & $5643(2)$ & $24(1)$ \\
\hline $\mathrm{P} 2$ & 2993(1) & 1893(4) & $6181(2)$ & $17(1)$ \\
\hline N1 & $2152(5)$ & $2414(14)$ & $7618(4)$ & $22(1)$ \\
\hline B1 & $2386(5)$ & $2187(15)$ & $8300(6)$ & $24(2)$ \\
\hline $\mathrm{C} 1$ & $1795(5)$ & $2650(20)$ & $7325(6)$ & $38(3)$ \\
\hline $\mathrm{C} 2$ & $1294(5)$ & $3040(30)$ & $7413(9)$ & $62(5)$ \\
\hline $\mathrm{C} 3$ & $2620(5)$ & $525(16)$ & $8262(9)$ & $32(2)$ \\
\hline $\mathrm{C} 4$ & $2301(7)$ & $-750(20)$ & $8125(13)$ & $61(5)$ \\
\hline C5 & $2784(5)$ & $3444(15)$ & $8418(7)$ & $28(2)$ \\
\hline C6 & $2619(7)$ & $5010(20)$ & $8433(14)$ & $59(4)$ \\
\hline $\mathrm{C} 7$ & $1955(5)$ & $2338(17)$ & $8801(7)$ & $24(2)$ \\
\hline $\mathrm{C} 8$ & 2096(8) & $2260(30)$ & 9494(9) & $53(4)$ \\
\hline C9 & $2499(4)$ & 2853(19) & $5082(6)$ & $24(2)$ \\
\hline $\mathrm{C} 10$ & $2885(4)$ & $1835(18)$ & $5312(4)$ & $26(2)$ \\
\hline $\mathrm{C} 11$ & $1583(3)$ & $1615(12)$ & $5250(6)$ & $25(2)$ \\
\hline $\mathrm{C} 12$ & $1775(5)$ & $45(13)$ & $5247(10)$ & $36(2)$ \\
\hline $\mathrm{C} 13$ & $1115(5)$ & $1650(20)$ & $5601(10)$ & $43(3)$ \\
\hline $\mathrm{C} 14$ & $1736(4)$ & $4706(10)$ & $5578(6)$ & $24(2)$ \\
\hline $\mathrm{C} 15$ & $2032(6)$ & $5885(17)$ & $5914(8)$ & $35(2)$ \\
\hline $\mathrm{C} 16$ & $1606(5)$ & $5207(16)$ & $4912(6)$ & $29(2)$ \\
\hline $\mathrm{C} 17$ & $3230(4)$ & $24(11)$ & $6331(7)$ & $31(2)$ \\
\hline $\mathrm{C} 18$ & $2844(5)$ & $-1159(17)$ & $6324(10)$ & $44(3)$ \\
\hline C19 & $3631(5)$ & $-470(20)$ & $5907(9)$ & $47(3)$ \\
\hline $\mathrm{C} 20$ & $3507(4)$ & $3087(13)$ & $6257(6)$ & $30(2)$ \\
\hline C21 & $3358(6)$ & $4716(14)$ & $6157(11)$ & $46(3)$ \\
\hline $\mathrm{C} 22$ & $3736(5)$ & $2950(20)$ & $6909(6)$ & $30(2)$ \\
\hline $\mathrm{Pd} 2$ & $226(1)$ & $7396(1)$ & $7875(1)$ & $19(1)$ \\
\hline
\end{tabular}




\begin{tabular}{|c|c|c|c|c|}
\hline P3 & $516(1)$ & $7663(5)$ & $8864(2)$ & $24(1)$ \\
\hline $\mathrm{P} 4$ & $-472(1)$ & $6616(4)$ & $8319(2)$ & $17(1)$ \\
\hline $\mathrm{N} 2$ & $364(4)$ & $7552(14)$ & $6903(4)$ & $22(1)$ \\
\hline B2 & $139(5)$ & $7393(15)$ & $6218(6)$ & $24(2)$ \\
\hline C31 & $715(4)$ & $7730(20)$ & $7224(6)$ & $38(3)$ \\
\hline C32 & $1210(5)$ & $8170(30)$ & $7085(9)$ & $62(5)$ \\
\hline C33 & $-263(5)$ & $8644(16)$ & $6192(8)$ & $32(2)$ \\
\hline C34 & $-93(8)$ & $10198(18)$ & $6222(15)$ & $61(5)$ \\
\hline C35 & $-89(5)$ & $5719(14)$ & $6182(8)$ & $28(2)$ \\
\hline C36 & $253(7)$ & $4460(20)$ & $6203(14)$ & $59(4)$ \\
\hline C37 & $566(5)$ & $7638(17)$ & $5711(7)$ & $24(2)$ \\
\hline C38 & $393(8)$ & $7730(30)$ & $5035(9)$ & $53(4)$ \\
\hline C39 & $3(4)$ & $7548(17)$ & $9400(6)$ & $24(2)$ \\
\hline $\mathrm{C} 40$ & $-360(4)$ & $6491(18)$ & $9185(4)$ & $26(2)$ \\
\hline $\mathrm{C} 41$ & $944(3)$ & $6391(12)$ & $9208(6)$ & $25(2)$ \\
\hline $\mathrm{C} 42$ & $762(5)$ & $4798(13)$ & $9213(10)$ & $36(2)$ \\
\hline $\mathrm{C} 43$ & $1401(5)$ & $6510(20)$ & $8835(10)$ & $43(3)$ \\
\hline $\mathrm{C} 44$ & $776(4)$ & $9504(11)$ & $8998(6)$ & $24(2)$ \\
\hline $\mathrm{C} 45$ & $465(6)$ & $10720(16)$ & $8718(8)$ & $35(2)$ \\
\hline $\mathrm{C} 46$ & $890(5)$ & $9886(16)$ & $9684(6)$ & $29(2)$ \\
\hline $\mathrm{C} 47$ & $-676(4)$ & $4786(11)$ & $8062(7)$ & $31(2)$ \\
\hline C48 & $-259(6)$ & $3734(19)$ & $8023(9)$ & $44(3)$ \\
\hline C49 & $-1066(5)$ & $4130(20)$ & $8464(10)$ & $47(3)$ \\
\hline $\mathrm{C} 50$ & $-1002(4)$ & $7761(13)$ & $8286(6)$ & $30(2)$ \\
\hline $\mathrm{C} 51$ & $-874(7)$ & $9375(14)$ & $8440(11)$ & $46(3)$ \\
\hline C52 & $-1253(5)$ & $7600(20)$ & $7649(6)$ & $30(2)$ \\
\hline
\end{tabular}


Table S3. Bond lengths $[\AA]$ and angles $\left[{ }^{\circ}\right]$ for jonlm07 (2a).

\begin{tabular}{|c|c|c|c|}
\hline $\operatorname{Pd}(1)-C(1)$ & $1.993(10)$ & $\mathrm{C}(8)-\mathrm{H}(8 \mathrm{~A})$ & 0.9800 \\
\hline $\operatorname{Pd}(1)-\mathrm{N}(1)$ & $2.101(8)$ & $\mathrm{C}(8)-\mathrm{H}(8 \mathrm{~B})$ & 0.9800 \\
\hline $\operatorname{Pd}(1)-\mathrm{P}(1)$ & $2.253(3)$ & $\mathrm{C}(8)-\mathrm{H}(8 \mathrm{C})$ & 0.9800 \\
\hline $\operatorname{Pd}(1)-P(2)$ & $2.353(3)$ & $\mathrm{C}(9)-\mathrm{C}(10)$ & $1.52(2)$ \\
\hline $\mathrm{P}(1)-\mathrm{C}(11)$ & $1.835(9)$ & $\mathrm{C}(9)-\mathrm{H}(9 \mathrm{~A})$ & 0.9900 \\
\hline$P(1)-C(14)$ & $1.849(9)$ & $\mathrm{C}(9)-\mathrm{H}(9 \mathrm{~B})$ & 0.9900 \\
\hline$P(1)-C(9)$ & $1.853(8)$ & $\mathrm{C}(10)-\mathrm{H}(10 \mathrm{~A})$ & 0.9900 \\
\hline$P(2)-C(20)$ & $1.833(9)$ & $\mathrm{C}(10)-\mathrm{H}(10 \mathrm{~B})$ & 0.9900 \\
\hline$P(2)-C(17)$ & $1.844(9)$ & $\mathrm{C}(11)-\mathrm{C}(12)$ & $1.520(10)$ \\
\hline $\mathrm{P}(2)-\mathrm{C}(10)$ & $1.863(9)$ & $\mathrm{C}(11)-\mathrm{C}(13)$ & $1.533(10)$ \\
\hline $\mathrm{N}(1)-\mathrm{C}(1)$ & $1.217(15)$ & $\mathrm{C}(11)-\mathrm{H}(11 \mathrm{~A})$ & 1.0000 \\
\hline $\mathrm{N}(1)-\mathrm{B}(1)$ & $1.602(14)$ & $\mathrm{C}(12)-\mathrm{H}(12 \mathrm{~A})$ & 0.9800 \\
\hline $\mathrm{B}(1)-\mathrm{C}(5)$ & $1.628(15)$ & $\mathrm{C}(12)-\mathrm{H}(12 \mathrm{~B})$ & 0.9800 \\
\hline $\mathrm{B}(1)-\mathrm{C}(7)$ & $1.635(14)$ & $\mathrm{C}(12)-\mathrm{H}(12 \mathrm{C})$ & 0.9800 \\
\hline $\mathrm{B}(1)-\mathrm{C}(3)$ & $1.643(16)$ & $\mathrm{C}(13)-\mathrm{H}(13 \mathrm{~A})$ & 0.9800 \\
\hline $\mathrm{C}(1)-\mathrm{C}(2)$ & $1.488(17)$ & $\mathrm{C}(13)-\mathrm{H}(13 \mathrm{~B})$ & 0.9800 \\
\hline $\mathrm{C}(2)-\mathrm{H}(2 \mathrm{~A})$ & 0.9800 & $\mathrm{C}(13)-\mathrm{H}(13 \mathrm{C})$ & 0.9800 \\
\hline $\mathrm{C}(2)-\mathrm{H}(2 \mathrm{~B})$ & 0.9800 & $C(14)-C(16)$ & $1.523(9)$ \\
\hline $\mathrm{C}(2)-\mathrm{H}(2 \mathrm{C})$ & 0.9800 & $C(14)-C(15)$ & $1.535(10)$ \\
\hline$C(3)-C(4)$ & $1.495(17)$ & $\mathrm{C}(14)-\mathrm{H}(14 \mathrm{~A})$ & 1.0000 \\
\hline $\mathrm{C}(3)-\mathrm{H}(3 \mathrm{~A})$ & 0.9900 & $\mathrm{C}(15)-\mathrm{H}(15 \mathrm{~A})$ & 0.9800 \\
\hline $\mathrm{C}(3)-\mathrm{H}(3 \mathrm{~B})$ & 0.9900 & $\mathrm{C}(15)-\mathrm{H}(15 \mathrm{~B})$ & 0.9800 \\
\hline $\mathrm{C}(4)-\mathrm{H}(4 \mathrm{~A})$ & 0.9800 & $\mathrm{C}(15)-\mathrm{H}(15 \mathrm{C})$ & 0.9800 \\
\hline $\mathrm{C}(4)-\mathrm{H}(4 \mathrm{~B})$ & 0.9800 & $\mathrm{C}(16)-\mathrm{H}(16 \mathrm{~A})$ & 0.9800 \\
\hline $\mathrm{C}(4)-\mathrm{H}(4 \mathrm{C})$ & 0.9800 & $\mathrm{C}(16)-\mathrm{H}(16 \mathrm{~B})$ & 0.9800 \\
\hline$C(5)-C(6)$ & $1.486(17)$ & $\mathrm{C}(16)-\mathrm{H}(16 \mathrm{C})$ & 0.9800 \\
\hline $\mathrm{C}(5)-\mathrm{H}(5 \mathrm{~A})$ & 0.9900 & $\mathrm{C}(17)-\mathrm{C}(19)$ & $1.524(10)$ \\
\hline $\mathrm{C}(5)-\mathrm{H}(5 \mathrm{~B})$ & 0.9900 & $\mathrm{C}(17)-\mathrm{C}(18)$ & $1.539(10)$ \\
\hline $\mathrm{C}(6)-\mathrm{H}(6 \mathrm{~A})$ & 0.9800 & $\mathrm{C}(17)-\mathrm{H}(17 \mathrm{~A})$ & 1.0000 \\
\hline $\mathrm{C}(6)-\mathrm{H}(6 \mathrm{~B})$ & 0.9800 & $\mathrm{C}(18)-\mathrm{H}(18 \mathrm{~A})$ & 0.9800 \\
\hline $\mathrm{C}(6)-\mathrm{H}(6 \mathrm{C})$ & 0.9800 & $\mathrm{C}(18)-\mathrm{H}(18 \mathrm{~B})$ & 0.9800 \\
\hline$C(7)-C(8)$ & $1.519(16)$ & $\mathrm{C}(18)-\mathrm{H}(18 \mathrm{C})$ & 0.9800 \\
\hline $\mathrm{C}(7)-\mathrm{H}(7 \mathrm{~A})$ & 0.9900 & C(19)-H(19A) & 0.9800 \\
\hline $\mathrm{C}(7)-\mathrm{H}(7 \mathrm{~B})$ & 0.9900 & C(19)-H(19B) & 0.9800 \\
\hline
\end{tabular}




\begin{tabular}{|c|c|c|c|}
\hline $\mathrm{C}(19)-\mathrm{H}(19 \mathrm{C})$ & 0.9800 & $\mathrm{C}(35)-\mathrm{H}(35 \mathrm{~A})$ & 0.9900 \\
\hline$C(20)-C(22)$ & $1.531(9)$ & $\mathrm{C}(35)-\mathrm{H}(35 \mathrm{~B})$ & 0.9900 \\
\hline$C(20)-C(21)$ & $1.544(10)$ & $\mathrm{C}(36)-\mathrm{H}(36 \mathrm{~A})$ & 0.9800 \\
\hline $\mathrm{C}(20)-\mathrm{H}(20 \mathrm{~A})$ & 1.0000 & $\mathrm{C}(36)-\mathrm{H}(36 \mathrm{~B})$ & 0.9800 \\
\hline $\mathrm{C}(21)-\mathrm{H}(21 \mathrm{~A})$ & 0.9800 & $\mathrm{C}(36)-\mathrm{H}(36 \mathrm{C})$ & 0.9800 \\
\hline $\mathrm{C}(21)-\mathrm{H}(21 \mathrm{~B})$ & 0.9800 & $C(37)-C(38)$ & $1.513(16)$ \\
\hline $\mathrm{C}(21)-\mathrm{H}(21 \mathrm{C})$ & 0.9800 & $\mathrm{C}(37)-\mathrm{H}(37 \mathrm{~A})$ & 0.9900 \\
\hline $\mathrm{C}(22)-\mathrm{H}(22 \mathrm{~A})$ & 0.9800 & $\mathrm{C}(37)-\mathrm{H}(37 \mathrm{~B})$ & 0.9900 \\
\hline $\mathrm{C}(22)-\mathrm{H}(22 \mathrm{~B})$ & 0.9800 & $\mathrm{C}(38)-\mathrm{H}(38 \mathrm{~A})$ & 0.9800 \\
\hline $\mathrm{C}(22)-\mathrm{H}(22 \mathrm{C})$ & 0.9800 & $\mathrm{C}(38)-\mathrm{H}(38 \mathrm{~B})$ & 0.9800 \\
\hline $\operatorname{Pd}(2)-C(31)$ & $1.989(10)$ & $\mathrm{C}(38)-\mathrm{H}(38 \mathrm{C})$ & 0.9800 \\
\hline $\operatorname{Pd}(2)-\mathrm{N}(2)$ & $2.096(8)$ & $C(39)-C(40)$ & $1.48(2)$ \\
\hline $\operatorname{Pd}(2)-P(3)$ & $2.260(3)$ & $\mathrm{C}(39)-\mathrm{H}(39 \mathrm{~A})$ & 0.9900 \\
\hline $\operatorname{Pd}(2)-P(4)$ & $2.321(3)$ & $\mathrm{C}(39)-\mathrm{H}(39 \mathrm{~B})$ & 0.9900 \\
\hline $\mathrm{P}(3)-\mathrm{C}(41)$ & $1.831(9)$ & $\mathrm{C}(40)-\mathrm{H}(40 \mathrm{~A})$ & 0.9900 \\
\hline $\mathrm{P}(3)-\mathrm{C}(44)$ & $1.842(9)$ & $\mathrm{C}(40)-\mathrm{H}(40 \mathrm{~B})$ & 0.9900 \\
\hline $\mathrm{P}(3)-\mathrm{C}(39)$ & $1.860(9)$ & $\mathrm{C}(41)-\mathrm{C}(42)$ & $1.529(10)$ \\
\hline $\mathrm{P}(4)-\mathrm{C}(47)$ & $1.833(9)$ & $\mathrm{C}(41)-\mathrm{C}(43)$ & $1.534(10)$ \\
\hline $\mathrm{P}(4)-\mathrm{C}(50)$ & $1.839(9)$ & $\mathrm{C}(41)-\mathrm{H}(41 \mathrm{~A})$ & 1.0000 \\
\hline $\mathrm{P}(4)-\mathrm{C}(40)$ & $1.860(9)$ & $\mathrm{C}(42)-\mathrm{H}(42 \mathrm{~A})$ & 0.9800 \\
\hline $\mathrm{N}(2)-\mathrm{C}(31)$ & $1.225(15)$ & $\mathrm{C}(42)-\mathrm{H}(42 \mathrm{~B})$ & 0.9800 \\
\hline $\mathrm{N}(2)-\mathrm{B}(2)$ & $1.589(15)$ & $\mathrm{C}(42)-\mathrm{H}(42 \mathrm{C})$ & 0.9800 \\
\hline $\mathrm{B}(2)-\mathrm{C}(33)$ & $1.613(15)$ & $\mathrm{C}(43)-\mathrm{H}(43 \mathrm{~A})$ & 0.9800 \\
\hline $\mathrm{B}(2)-\mathrm{C}(37)$ & $1.643(14)$ & $\mathrm{C}(43)-\mathrm{H}(43 \mathrm{~B})$ & 0.9800 \\
\hline $\mathrm{B}(2)-\mathrm{C}(35)$ & $1.647(15)$ & $\mathrm{C}(43)-\mathrm{H}(43 \mathrm{C})$ & 0.9800 \\
\hline $\mathrm{C}(31)-\mathrm{C}(32)$ & $1.501(16)$ & $C(44)-C(46)$ & $1.525(9)$ \\
\hline $\mathrm{C}(32)-\mathrm{H}(32 \mathrm{~A})$ & 0.9800 & $C(44)-C(45)$ & $1.533(10)$ \\
\hline $\mathrm{C}(32)-\mathrm{H}(32 \mathrm{~B})$ & 0.9800 & $\mathrm{C}(44)-\mathrm{H}(44 \mathrm{~A})$ & 1.0000 \\
\hline $\mathrm{C}(32)-\mathrm{H}(32 \mathrm{C})$ & 0.9800 & $\mathrm{C}(45)-\mathrm{H}(45 \mathrm{~A})$ & 0.9800 \\
\hline$C(33)-C(34)$ & $1.484(17)$ & $\mathrm{C}(45)-\mathrm{H}(45 \mathrm{~B})$ & 0.9800 \\
\hline $\mathrm{C}(33)-\mathrm{H}(33 \mathrm{~A})$ & 0.9900 & $\mathrm{C}(45)-\mathrm{H}(45 \mathrm{C})$ & 0.9800 \\
\hline $\mathrm{C}(33)-\mathrm{H}(33 \mathrm{~B})$ & 0.9900 & $\mathrm{C}(46)-\mathrm{H}(46 \mathrm{~A})$ & 0.9800 \\
\hline $\mathrm{C}(34)-\mathrm{H}(34 \mathrm{~A})$ & 0.9800 & $\mathrm{C}(46)-\mathrm{H}(46 \mathrm{~B})$ & 0.9800 \\
\hline $\mathrm{C}(34)-\mathrm{H}(34 \mathrm{~B})$ & 0.9800 & $\mathrm{C}(46)-\mathrm{H}(46 \mathrm{C})$ & 0.9800 \\
\hline $\mathrm{C}(34)-\mathrm{H}(34 \mathrm{C})$ & 0.9800 & $\mathrm{C}(47)-\mathrm{C}(49)$ & $1.523(10)$ \\
\hline$C(35)-C(36)$ & $1.500(17)$ & $\mathrm{C}(47)-\mathrm{C}(48)$ & $1.529(10)$ \\
\hline
\end{tabular}




\begin{tabular}{|c|c|c|c|}
\hline $\mathrm{C}(47)-\mathrm{H}(47 \mathrm{~A})$ & 1.0000 & $\mathrm{~B}(1)-\mathrm{N}(1)-\mathrm{Pd}(1)$ & $145.7(9)$ \\
\hline $\mathrm{C}(48)-\mathrm{H}(48 \mathrm{~A})$ & 0.9800 & $\mathrm{~N}(1)-\mathrm{B}(1)-\mathrm{C}(5)$ & $110.1(11)$ \\
\hline $\mathrm{C}(48)-\mathrm{H}(48 \mathrm{~B})$ & 0.9800 & $\mathrm{~N}(1)-\mathrm{B}(1)-\mathrm{C}(7)$ & $104.7(10)$ \\
\hline $\mathrm{C}(48)-\mathrm{H}(48 \mathrm{C})$ & 0.9800 & $\mathrm{C}(5)-\mathrm{B}(1)-\mathrm{C}(7)$ & $111.9(11)$ \\
\hline $\mathrm{C}(49)-\mathrm{H}(49 \mathrm{~A})$ & 0.9800 & $\mathrm{~N}(1)-\mathrm{B}(1)-\mathrm{C}(3)$ & $104.1(11)$ \\
\hline $\mathrm{C}(49)-\mathrm{H}(49 \mathrm{~B})$ & 0.9800 & $\mathrm{C}(5)-\mathrm{B}(1)-\mathrm{C}(3)$ & $111.0(11)$ \\
\hline $\mathrm{C}(49)-\mathrm{H}(49 \mathrm{C})$ & 0.9800 & $\mathrm{C}(7)-\mathrm{B}(1)-\mathrm{C}(3)$ & $114.6(11)$ \\
\hline $\mathrm{C}(50)-\mathrm{C}(52)$ & $1.532(9)$ & $\mathrm{N}(1)-\mathrm{C}(1)-\mathrm{C}(2)$ & $142.3(13)$ \\
\hline$C(50)-C(51)$ & $1.535(10)$ & $\mathrm{N}(1)-\mathrm{C}(1)-\mathrm{Pd}(1)$ & $77.7(6)$ \\
\hline $\mathrm{C}(50)-\mathrm{H}(50 \mathrm{~A})$ & 1.0000 & $C(2)-C(1)-P d(1)$ & $139.8(12)$ \\
\hline $\mathrm{C}(51)-\mathrm{H}(51 \mathrm{~A})$ & 0.9800 & $\mathrm{C}(1)-\mathrm{C}(2)-\mathrm{H}(2 \mathrm{~A})$ & 109.5 \\
\hline $\mathrm{C}(51)-\mathrm{H}(51 \mathrm{~B})$ & 0.9800 & $\mathrm{C}(1)-\mathrm{C}(2)-\mathrm{H}(2 \mathrm{~B})$ & 109.5 \\
\hline $\mathrm{C}(51)-\mathrm{H}(51 \mathrm{C})$ & 0.9800 & $\mathrm{H}(2 \mathrm{~A})-\mathrm{C}(2)-\mathrm{H}(2 \mathrm{~B})$ & 109.5 \\
\hline $\mathrm{C}(52)-\mathrm{H}(52 \mathrm{~A})$ & 0.9800 & $\mathrm{C}(1)-\mathrm{C}(2)-\mathrm{H}(2 \mathrm{C})$ & 109.5 \\
\hline $\mathrm{C}(52)-\mathrm{H}(52 \mathrm{~B})$ & 0.9800 & $\mathrm{H}(2 \mathrm{~A})-\mathrm{C}(2)-\mathrm{H}(2 \mathrm{C})$ & 109.5 \\
\hline $\mathrm{C}(52)-\mathrm{H}(52 \mathrm{C})$ & 0.9800 & $\mathrm{H}(2 \mathrm{~B})-\mathrm{C}(2)-\mathrm{H}(2 \mathrm{C})$ & 109.5 \\
\hline $\mathrm{C}(1)-\mathrm{Pd}(1)-\mathrm{N}(1)$ & $34.5(4)$ & $\mathrm{C}(4)-\mathrm{C}(3)-\mathrm{B}(1)$ & $117.5(13)$ \\
\hline $\mathrm{C}(1)-\mathrm{Pd}(1)-\mathrm{P}(1)$ & $116.1(5)$ & $\mathrm{C}(4)-\mathrm{C}(3)-\mathrm{H}(3 \mathrm{~A})$ & 107.9 \\
\hline $\mathrm{N}(1)-\mathrm{Pd}(1)-\mathrm{P}(1)$ & $150.4(4)$ & $\mathrm{B}(1)-\mathrm{C}(3)-\mathrm{H}(3 \mathrm{~A})$ & 107.9 \\
\hline $\mathrm{C}(1)-\mathrm{Pd}(1)-\mathrm{P}(2)$ & $156.5(5)$ & $\mathrm{C}(4)-\mathrm{C}(3)-\mathrm{H}(3 \mathrm{~B})$ & 107.9 \\
\hline $\mathrm{N}(1)-\mathrm{Pd}(1)-\mathrm{P}(2)$ & $122.3(4)$ & $\mathrm{B}(1)-\mathrm{C}(3)-\mathrm{H}(3 \mathrm{~B})$ & 107.9 \\
\hline$P(1)-P d(1)-P(2)$ & $87.27(14)$ & $\mathrm{H}(3 \mathrm{~A})-\mathrm{C}(3)-\mathrm{H}(3 \mathrm{~B})$ & 107.2 \\
\hline $\mathrm{C}(11)-\mathrm{P}(1)-\mathrm{C}(14)$ & $104.1(5)$ & $\mathrm{C}(3)-\mathrm{C}(4)-\mathrm{H}(4 \mathrm{~A})$ & 109.5 \\
\hline $\mathrm{C}(11)-\mathrm{P}(1)-\mathrm{C}(9)$ & $102.6(7)$ & $\mathrm{C}(3)-\mathrm{C}(4)-\mathrm{H}(4 \mathrm{~B})$ & 109.5 \\
\hline $\mathrm{C}(14)-\mathrm{P}(1)-\mathrm{C}(9)$ & $105.5(7)$ & $\mathrm{H}(4 \mathrm{~A})-\mathrm{C}(4)-\mathrm{H}(4 \mathrm{~B})$ & 109.5 \\
\hline $\mathrm{C}(11)-\mathrm{P}(1)-\mathrm{Pd}(1)$ & $122.8(5)$ & $\mathrm{C}(3)-\mathrm{C}(4)-\mathrm{H}(4 \mathrm{C})$ & 109.5 \\
\hline $\mathrm{C}(14)-\mathrm{P}(1)-\mathrm{Pd}(1)$ & $110.6(4)$ & $\mathrm{H}(4 \mathrm{~A})-\mathrm{C}(4)-\mathrm{H}(4 \mathrm{C})$ & 109.5 \\
\hline $\mathrm{C}(9)-\mathrm{P}(1)-\mathrm{Pd}(1)$ & $109.8(5)$ & $\mathrm{H}(4 \mathrm{~B})-\mathrm{C}(4)-\mathrm{H}(4 \mathrm{C})$ & 109.5 \\
\hline $\mathrm{C}(20)-\mathrm{P}(2)-\mathrm{C}(17)$ & $103.0(6)$ & $\mathrm{C}(6)-\mathrm{C}(5)-\mathrm{B}(1)$ & $116.1(13)$ \\
\hline $\mathrm{C}(20)-\mathrm{P}(2)-\mathrm{C}(10)$ & $103.7(6)$ & $\mathrm{C}(6)-\mathrm{C}(5)-\mathrm{H}(5 \mathrm{~A})$ & 108.2 \\
\hline $\mathrm{C}(17)-\mathrm{P}(2)-\mathrm{C}(10)$ & $101.9(7)$ & $\mathrm{B}(1)-\mathrm{C}(5)-\mathrm{H}(5 \mathrm{~A})$ & 108.2 \\
\hline$C(20)-P(2)-P d(1)$ & $123.7(4)$ & $\mathrm{C}(6)-\mathrm{C}(5)-\mathrm{H}(5 \mathrm{~B})$ & 108.2 \\
\hline$C(17)-P(2)-P d(1)$ & $116.9(4)$ & $\mathrm{B}(1)-\mathrm{C}(5)-\mathrm{H}(5 \mathrm{~B})$ & 108.2 \\
\hline$C(10)-P(2)-P d(1)$ & $105.0(4)$ & $\mathrm{H}(5 \mathrm{~A})-\mathrm{C}(5)-\mathrm{H}(5 \mathrm{~B})$ & 107.4 \\
\hline $\mathrm{C}(1)-\mathrm{N}(1)-\mathrm{B}(1)$ & $146.4(11)$ & $\mathrm{C}(5)-\mathrm{C}(6)-\mathrm{H}(6 \mathrm{~A})$ & 109.5 \\
\hline $\mathrm{C}(1)-\mathrm{N}(1)-\mathrm{Pd}(1)$ & $67.9(6)$ & $\mathrm{C}(5)-\mathrm{C}(6)-\mathrm{H}(6 \mathrm{~B})$ & 109.5 \\
\hline
\end{tabular}




\begin{tabular}{|c|c|c|c|}
\hline $\mathrm{H}(6 \mathrm{~A})-\mathrm{C}(6)-\mathrm{H}(6 \mathrm{~B})$ & 109.5 & $\mathrm{H}(12 \mathrm{~A})-\mathrm{C}(12)-\mathrm{H}(12 \mathrm{~B})$ & 109.5 \\
\hline $\mathrm{C}(5)-\mathrm{C}(6)-\mathrm{H}(6 \mathrm{C})$ & 109.5 & $\mathrm{C}(11)-\mathrm{C}(12)-\mathrm{H}(12 \mathrm{C})$ & 109.5 \\
\hline $\mathrm{H}(6 \mathrm{~A})-\mathrm{C}(6)-\mathrm{H}(6 \mathrm{C})$ & 109.5 & $\mathrm{H}(12 \mathrm{~A})-\mathrm{C}(12)-\mathrm{H}(12 \mathrm{C})$ & 109.5 \\
\hline $\mathrm{H}(6 \mathrm{~B})-\mathrm{C}(6)-\mathrm{H}(6 \mathrm{C})$ & 109.5 & $\mathrm{H}(12 \mathrm{~B})-\mathrm{C}(12)-\mathrm{H}(12 \mathrm{C})$ & 109.5 \\
\hline $\mathrm{C}(8)-\mathrm{C}(7)-\mathrm{B}(1)$ & $114.7(14)$ & $\mathrm{C}(11)-\mathrm{C}(13)-\mathrm{H}(13 \mathrm{~A})$ & 109.5 \\
\hline $\mathrm{C}(8)-\mathrm{C}(7)-\mathrm{H}(7 \mathrm{~A})$ & 108.6 & $\mathrm{C}(11)-\mathrm{C}(13)-\mathrm{H}(13 \mathrm{~B})$ & 109.5 \\
\hline $\mathrm{B}(1)-\mathrm{C}(7)-\mathrm{H}(7 \mathrm{~A})$ & 108.6 & $\mathrm{H}(13 \mathrm{~A})-\mathrm{C}(13)-\mathrm{H}(13 \mathrm{~B})$ & 109.5 \\
\hline $\mathrm{C}(8)-\mathrm{C}(7)-\mathrm{H}(7 \mathrm{~B})$ & 108.6 & $\mathrm{C}(11)-\mathrm{C}(13)-\mathrm{H}(13 \mathrm{C})$ & 109.5 \\
\hline $\mathrm{B}(1)-\mathrm{C}(7)-\mathrm{H}(7 \mathrm{~B})$ & 108.6 & $\mathrm{H}(13 \mathrm{~A})-\mathrm{C}(13)-\mathrm{H}(13 \mathrm{C})$ & 109.5 \\
\hline $\mathrm{H}(7 \mathrm{~A})-\mathrm{C}(7)-\mathrm{H}(7 \mathrm{~B})$ & 107.6 & H(13B)-C(13)-H(13C) & 109.5 \\
\hline $\mathrm{C}(7)-\mathrm{C}(8)-\mathrm{H}(8 \mathrm{~A})$ & 109.5 & $C(16)-C(14)-C(15)$ & $110.9(11)$ \\
\hline $\mathrm{C}(7)-\mathrm{C}(8)-\mathrm{H}(8 \mathrm{~B})$ & 109.5 & $\mathrm{C}(16)-\mathrm{C}(14)-\mathrm{P}(1)$ & $116.0(8)$ \\
\hline $\mathrm{H}(8 \mathrm{~A})-\mathrm{C}(8)-\mathrm{H}(8 \mathrm{~B})$ & 109.5 & $\mathrm{C}(15)-\mathrm{C}(14)-\mathrm{P}(1)$ & $111.4(9)$ \\
\hline $\mathrm{C}(7)-\mathrm{C}(8)-\mathrm{H}(8 \mathrm{C})$ & 109.5 & $\mathrm{C}(16)-\mathrm{C}(14)-\mathrm{H}(14 \mathrm{~A})$ & 105.9 \\
\hline $\mathrm{H}(8 \mathrm{~A})-\mathrm{C}(8)-\mathrm{H}(8 \mathrm{C})$ & 109.5 & $\mathrm{C}(15)-\mathrm{C}(14)-\mathrm{H}(14 \mathrm{~A})$ & 105.9 \\
\hline $\mathrm{H}(8 \mathrm{~B})-\mathrm{C}(8)-\mathrm{H}(8 \mathrm{C})$ & 109.5 & $\mathrm{P}(1)-\mathrm{C}(14)-\mathrm{H}(14 \mathrm{~A})$ & 105.9 \\
\hline $\mathrm{C}(10)-\mathrm{C}(9)-\mathrm{P}(1)$ & $110.7(8)$ & $\mathrm{C}(14)-\mathrm{C}(15)-\mathrm{H}(15 \mathrm{~A})$ & 109.5 \\
\hline $\mathrm{C}(10)-\mathrm{C}(9)-\mathrm{H}(9 \mathrm{~A})$ & 109.5 & $\mathrm{C}(14)-\mathrm{C}(15)-\mathrm{H}(15 \mathrm{~B})$ & 109.5 \\
\hline $\mathrm{P}(1)-\mathrm{C}(9)-\mathrm{H}(9 \mathrm{~A})$ & 109.5 & $\mathrm{H}(15 \mathrm{~A})-\mathrm{C}(15)-\mathrm{H}(15 \mathrm{~B})$ & 109.5 \\
\hline $\mathrm{C}(10)-\mathrm{C}(9)-\mathrm{H}(9 \mathrm{~B})$ & 109.5 & $\mathrm{C}(14)-\mathrm{C}(15)-\mathrm{H}(15 \mathrm{C})$ & 109.5 \\
\hline $\mathrm{P}(1)-\mathrm{C}(9)-\mathrm{H}(9 \mathrm{~B})$ & 109.5 & $\mathrm{H}(15 \mathrm{~A})-\mathrm{C}(15)-\mathrm{H}(15 \mathrm{C})$ & 109.5 \\
\hline $\mathrm{H}(9 \mathrm{~A})-\mathrm{C}(9)-\mathrm{H}(9 \mathrm{~B})$ & 108.1 & $\mathrm{H}(15 \mathrm{~B})-\mathrm{C}(15)-\mathrm{H}(15 \mathrm{C})$ & 109.5 \\
\hline $\mathrm{C}(9)-\mathrm{C}(10)-\mathrm{P}(2)$ & $114.9(9)$ & $\mathrm{C}(14)-\mathrm{C}(16)-\mathrm{H}(16 \mathrm{~A})$ & 109.5 \\
\hline $\mathrm{C}(9)-\mathrm{C}(10)-\mathrm{H}(10 \mathrm{~A})$ & 108.6 & $\mathrm{C}(14)-\mathrm{C}(16)-\mathrm{H}(16 \mathrm{~B})$ & 109.5 \\
\hline $\mathrm{P}(2)-\mathrm{C}(10)-\mathrm{H}(10 \mathrm{~A})$ & 108.6 & $\mathrm{H}(16 \mathrm{~A})-\mathrm{C}(16)-\mathrm{H}(16 \mathrm{~B})$ & 109.5 \\
\hline $\mathrm{C}(9)-\mathrm{C}(10)-\mathrm{H}(10 \mathrm{~B})$ & 108.6 & $\mathrm{C}(14)-\mathrm{C}(16)-\mathrm{H}(16 \mathrm{C})$ & 109.5 \\
\hline $\mathrm{P}(2)-\mathrm{C}(10)-\mathrm{H}(10 \mathrm{~B})$ & 108.6 & $\mathrm{H}(16 \mathrm{~A})-\mathrm{C}(16)-\mathrm{H}(16 \mathrm{C})$ & 109.5 \\
\hline $\mathrm{H}(10 \mathrm{~A})-\mathrm{C}(10)-\mathrm{H}(10 \mathrm{~B})$ & 107.5 & $\mathrm{H}(16 \mathrm{~B})-\mathrm{C}(16)-\mathrm{H}(16 \mathrm{C})$ & 109.5 \\
\hline $\mathrm{C}(12)-\mathrm{C}(11)-\mathrm{C}(13)$ & $109.7(13)$ & $\mathrm{C}(19)-\mathrm{C}(17)-\mathrm{C}(18)$ & $109.6(12)$ \\
\hline $\mathrm{C}(12)-\mathrm{C}(11)-\mathrm{P}(1)$ & 109.1(9) & $\mathrm{C}(19)-\mathrm{C}(17)-\mathrm{P}(2)$ & $116.4(11)$ \\
\hline $\mathrm{C}(13)-\mathrm{C}(11)-\mathrm{P}(1)$ & $110.1(10)$ & $\mathrm{C}(18)-\mathrm{C}(17)-\mathrm{P}(2)$ & 111.5(9) \\
\hline $\mathrm{C}(12)-\mathrm{C}(11)-\mathrm{H}(11 \mathrm{~A})$ & 109.3 & $\mathrm{C}(19)-\mathrm{C}(17)-\mathrm{H}(17 \mathrm{~A})$ & 106.2 \\
\hline $\mathrm{C}(13)-\mathrm{C}(11)-\mathrm{H}(11 \mathrm{~A})$ & 109.3 & $\mathrm{C}(18)-\mathrm{C}(17)-\mathrm{H}(17 \mathrm{~A})$ & 106.2 \\
\hline$P(1)-C(11)-H(11 A)$ & 109.3 & $\mathrm{P}(2)-\mathrm{C}(17)-\mathrm{H}(17 \mathrm{~A})$ & 106.2 \\
\hline $\mathrm{C}(11)-\mathrm{C}(12)-\mathrm{H}(12 \mathrm{~A})$ & 109.5 & $\mathrm{C}(17)-\mathrm{C}(18)-\mathrm{H}(18 \mathrm{~A})$ & 109.5 \\
\hline $\mathrm{C}(11)-\mathrm{C}(12)-\mathrm{H}(12 \mathrm{~B})$ & 109.5 & $\mathrm{C}(17)-\mathrm{C}(18)-\mathrm{H}(18 \mathrm{~B})$ & 109.5 \\
\hline
\end{tabular}




\begin{tabular}{|c|c|c|c|}
\hline $\mathrm{H}(18 \mathrm{~A})-\mathrm{C}(18)-\mathrm{H}(18 \mathrm{~B})$ & 109.5 & $\mathrm{C}(44)-\mathrm{P}(3)-\mathrm{C}(39)$ & $106.1(7)$ \\
\hline $\mathrm{C}(17)-\mathrm{C}(18)-\mathrm{H}(18 \mathrm{C})$ & 109.5 & $\mathrm{C}(41)-\mathrm{P}(3)-\mathrm{Pd}(2)$ & $123.2(5)$ \\
\hline $\mathrm{H}(18 \mathrm{~A})-\mathrm{C}(18)-\mathrm{H}(18 \mathrm{C})$ & 109.5 & $\mathrm{C}(44)-\mathrm{P}(3)-\mathrm{Pd}(2)$ & $112.8(4)$ \\
\hline $\mathrm{H}(18 \mathrm{~B})-\mathrm{C}(18)-\mathrm{H}(18 \mathrm{C})$ & 109.5 & $\mathrm{C}(39)-\mathrm{P}(3)-\mathrm{Pd}(2)$ & $105.4(5)$ \\
\hline $\mathrm{C}(17)-\mathrm{C}(19)-\mathrm{H}(19 \mathrm{~A})$ & 109.5 & $\mathrm{C}(47)-\mathrm{P}(4)-\mathrm{C}(50)$ & $103.4(5)$ \\
\hline $\mathrm{C}(17)-\mathrm{C}(19)-\mathrm{H}(19 \mathrm{~B})$ & 109.5 & $\mathrm{C}(47)-\mathrm{P}(4)-\mathrm{C}(40)$ & $107.0(7)$ \\
\hline $\mathrm{H}(19 \mathrm{~A})-\mathrm{C}(19)-\mathrm{H}(19 \mathrm{~B})$ & 109.5 & $C(50)-P(4)-C(40)$ & $102.4(6)$ \\
\hline $\mathrm{C}(17)-\mathrm{C}(19)-\mathrm{H}(19 \mathrm{C})$ & 109.5 & $C(47)-P(4)-P d(2)$ & $115.4(4)$ \\
\hline$H(19 A)-C(19)-H(19 C)$ & 109.5 & $C(50)-P(4)-P d(2)$ & $121.8(5)$ \\
\hline $\mathrm{H}(19 \mathrm{~B})-\mathrm{C}(19)-\mathrm{H}(19 \mathrm{C})$ & 109.5 & $C(40)-P(4)-P d(2)$ & $105.4(4)$ \\
\hline$C(22)-C(20)-C(21)$ & $108.5(12)$ & $\mathrm{C}(31)-\mathrm{N}(2)-\mathrm{B}(2)$ & $148.1(11)$ \\
\hline $\mathrm{C}(22)-\mathrm{C}(20)-\mathrm{P}(2)$ & $112.0(9)$ & $\mathrm{C}(31)-\mathrm{N}(2)-\mathrm{Pd}(2)$ & $67.8(6)$ \\
\hline $\mathrm{C}(21)-\mathrm{C}(20)-\mathrm{P}(2)$ & $109.0(9)$ & $\mathrm{B}(2)-\mathrm{N}(2)-\mathrm{Pd}(2)$ & $143.9(9)$ \\
\hline$C(22)-C(20)-H(20 A)$ & 109.1 & $\mathrm{~N}(2)-\mathrm{B}(2)-\mathrm{C}(33)$ & $104.9(11)$ \\
\hline$C(21)-C(20)-H(20 A)$ & 109.1 & $\mathrm{~N}(2)-\mathrm{B}(2)-\mathrm{C}(37)$ & $106.1(10)$ \\
\hline$P(2)-C(20)-H(20 A)$ & 109.1 & $\mathrm{C}(33)-\mathrm{B}(2)-\mathrm{C}(37)$ & $114.6(11)$ \\
\hline $\mathrm{C}(20)-\mathrm{C}(21)-\mathrm{H}(21 \mathrm{~A})$ & 109.5 & $\mathrm{~N}(2)-\mathrm{B}(2)-\mathrm{C}(35)$ & $106.6(10)$ \\
\hline $\mathrm{C}(20)-\mathrm{C}(21)-\mathrm{H}(21 \mathrm{~B})$ & 109.5 & $\mathrm{C}(33)-\mathrm{B}(2)-\mathrm{C}(35)$ & $110.9(10)$ \\
\hline $\mathrm{H}(21 \mathrm{~A})-\mathrm{C}(21)-\mathrm{H}(21 \mathrm{~B})$ & 109.5 & $\mathrm{C}(37)-\mathrm{B}(2)-\mathrm{C}(35)$ & $112.9(11)$ \\
\hline $\mathrm{C}(20)-\mathrm{C}(21)-\mathrm{H}(21 \mathrm{C})$ & 109.5 & $\mathrm{~N}(2)-\mathrm{C}(31)-\mathrm{C}(32)$ & $134.8(13)$ \\
\hline $\mathrm{H}(21 \mathrm{~A})-\mathrm{C}(21)-\mathrm{H}(21 \mathrm{C})$ & 109.5 & $\mathrm{~N}(2)-\mathrm{C}(31)-\mathrm{Pd}(2)$ & $77.4(6)$ \\
\hline $\mathrm{H}(21 \mathrm{~B})-\mathrm{C}(21)-\mathrm{H}(21 \mathrm{C})$ & 109.5 & $C(32)-C(31)-P d(2)$ & $147.4(12)$ \\
\hline $\mathrm{C}(20)-\mathrm{C}(22)-\mathrm{H}(22 \mathrm{~A})$ & 109.5 & $\mathrm{C}(31)-\mathrm{C}(32)-\mathrm{H}(32 \mathrm{~A})$ & 109.5 \\
\hline $\mathrm{C}(20)-\mathrm{C}(22)-\mathrm{H}(22 \mathrm{~B})$ & 109.5 & $\mathrm{C}(31)-\mathrm{C}(32)-\mathrm{H}(32 \mathrm{~B})$ & 109.5 \\
\hline $\mathrm{H}(22 \mathrm{~A})-\mathrm{C}(22)-\mathrm{H}(22 \mathrm{~B})$ & 109.5 & $\mathrm{H}(32 \mathrm{~A})-\mathrm{C}(32)-\mathrm{H}(32 \mathrm{~B})$ & 109.5 \\
\hline $\mathrm{C}(20)-\mathrm{C}(22)-\mathrm{H}(22 \mathrm{C})$ & 109.5 & $\mathrm{C}(31)-\mathrm{C}(32)-\mathrm{H}(32 \mathrm{C})$ & 109.5 \\
\hline $\mathrm{H}(22 \mathrm{~A})-\mathrm{C}(22)-\mathrm{H}(22 \mathrm{C})$ & 109.5 & $\mathrm{H}(32 \mathrm{~A})-\mathrm{C}(32)-\mathrm{H}(32 \mathrm{C})$ & 109.5 \\
\hline $\mathrm{H}(22 \mathrm{~B})-\mathrm{C}(22)-\mathrm{H}(22 \mathrm{C})$ & 109.5 & $\mathrm{H}(32 \mathrm{~B})-\mathrm{C}(32)-\mathrm{H}(32 \mathrm{C})$ & 109.5 \\
\hline $\mathrm{C}(31)-\mathrm{Pd}(2)-\mathrm{N}(2)$ & $34.8(4)$ & $\mathrm{C}(34)-\mathrm{C}(33)-\mathrm{B}(2)$ & $115.1(14)$ \\
\hline $\mathrm{C}(31)-\mathrm{Pd}(2)-\mathrm{P}(3)$ & $111.3(5)$ & $\mathrm{C}(34)-\mathrm{C}(33)-\mathrm{H}(33 \mathrm{~A})$ & 108.5 \\
\hline $\mathrm{N}(2)-\mathrm{Pd}(2)-\mathrm{P}(3)$ & $145.9(4)$ & $\mathrm{B}(2)-\mathrm{C}(33)-\mathrm{H}(33 \mathrm{~A})$ & 108.5 \\
\hline$C(31)-P d(2)-P(4)$ & $159.2(5)$ & $\mathrm{C}(34)-\mathrm{C}(33)-\mathrm{H}(33 \mathrm{~B})$ & 108.5 \\
\hline $\mathrm{N}(2)-\mathrm{Pd}(2)-\mathrm{P}(4)$ & $125.4(4)$ & $\mathrm{B}(2)-\mathrm{C}(33)-\mathrm{H}(33 \mathrm{~B})$ & 108.5 \\
\hline$P(3)-P d(2)-P(4)$ & $88.68(14)$ & $\mathrm{H}(33 \mathrm{~A})-\mathrm{C}(33)-\mathrm{H}(33 \mathrm{~B})$ & 107.5 \\
\hline $\mathrm{C}(41)-\mathrm{P}(3)-\mathrm{C}(44)$ & $103.4(5)$ & $\mathrm{C}(33)-\mathrm{C}(34)-\mathrm{H}(34 \mathrm{~A})$ & 109.5 \\
\hline $\mathrm{C}(41)-\mathrm{P}(3)-\mathrm{C}(39)$ & $104.7(6)$ & $\mathrm{C}(33)-\mathrm{C}(34)-\mathrm{H}(34 \mathrm{~B})$ & 109.5 \\
\hline
\end{tabular}




\begin{tabular}{|c|c|c|c|}
\hline $\mathrm{H}(34 \mathrm{~A})-\mathrm{C}(34)-\mathrm{H}(34 \mathrm{~B})$ & 109.5 & $\mathrm{P}(4)-\mathrm{C}(40)-\mathrm{H}(40 \mathrm{~A})$ & 109.1 \\
\hline $\mathrm{C}(33)-\mathrm{C}(34)-\mathrm{H}(34 \mathrm{C})$ & 109.5 & $\mathrm{C}(39)-\mathrm{C}(40)-\mathrm{H}(40 \mathrm{~B})$ & 109.1 \\
\hline $\mathrm{H}(34 \mathrm{~A})-\mathrm{C}(34)-\mathrm{H}(34 \mathrm{C})$ & 109.5 & $\mathrm{P}(4)-\mathrm{C}(40)-\mathrm{H}(40 \mathrm{~B})$ & 109.1 \\
\hline $\mathrm{H}(34 \mathrm{~B})-\mathrm{C}(34)-\mathrm{H}(34 \mathrm{C})$ & 109.5 & $\mathrm{H}(40 \mathrm{~A})-\mathrm{C}(40)-\mathrm{H}(40 \mathrm{~B})$ & 107.8 \\
\hline $\mathrm{C}(36)-\mathrm{C}(35)-\mathrm{B}(2)$ & $115.6(12)$ & $\mathrm{C}(42)-\mathrm{C}(41)-\mathrm{C}(43)$ & $111.3(12)$ \\
\hline $\mathrm{C}(36)-\mathrm{C}(35)-\mathrm{H}(35 \mathrm{~A})$ & 108.4 & $\mathrm{C}(42)-\mathrm{C}(41)-\mathrm{P}(3)$ & 111.3(9) \\
\hline $\mathrm{B}(2)-\mathrm{C}(35)-\mathrm{H}(35 \mathrm{~A})$ & 108.4 & $\mathrm{C}(43)-\mathrm{C}(41)-\mathrm{P}(3)$ & $108.9(10)$ \\
\hline $\mathrm{C}(36)-\mathrm{C}(35)-\mathrm{H}(35 \mathrm{~B})$ & 108.4 & $\mathrm{C}(42)-\mathrm{C}(41)-\mathrm{H}(41 \mathrm{~A})$ & 108.4 \\
\hline $\mathrm{B}(2)-\mathrm{C}(35)-\mathrm{H}(35 \mathrm{~B})$ & 108.4 & $\mathrm{C}(43)-\mathrm{C}(41)-\mathrm{H}(41 \mathrm{~A})$ & 108.4 \\
\hline $\mathrm{H}(35 \mathrm{~A})-\mathrm{C}(35)-\mathrm{H}(35 \mathrm{~B})$ & 107.4 & $\mathrm{P}(3)-\mathrm{C}(41)-\mathrm{H}(41 \mathrm{~A})$ & 108.4 \\
\hline$C(35)-C(36)-H(36 A)$ & 109.5 & $\mathrm{C}(41)-\mathrm{C}(42)-\mathrm{H}(42 \mathrm{~A})$ & 109.5 \\
\hline $\mathrm{C}(35)-\mathrm{C}(36)-\mathrm{H}(36 \mathrm{~B})$ & 109.5 & $\mathrm{C}(41)-\mathrm{C}(42)-\mathrm{H}(42 \mathrm{~B})$ & 109.5 \\
\hline$H(36 A)-C(36)-H(36 B)$ & 109.5 & $\mathrm{H}(42 \mathrm{~A})-\mathrm{C}(42)-\mathrm{H}(42 \mathrm{~B})$ & 109.5 \\
\hline$C(35)-C(36)-H(36 C)$ & 109.5 & $\mathrm{C}(41)-\mathrm{C}(42)-\mathrm{H}(42 \mathrm{C})$ & 109.5 \\
\hline $\mathrm{H}(36 \mathrm{~A})-\mathrm{C}(36)-\mathrm{H}(36 \mathrm{C})$ & 109.5 & $\mathrm{H}(42 \mathrm{~A})-\mathrm{C}(42)-\mathrm{H}(42 \mathrm{C})$ & 109.5 \\
\hline $\mathrm{H}(36 \mathrm{~B})-\mathrm{C}(36)-\mathrm{H}(36 \mathrm{C})$ & 109.5 & $\mathrm{H}(42 \mathrm{~B})-\mathrm{C}(42)-\mathrm{H}(42 \mathrm{C})$ & 109.5 \\
\hline $\mathrm{C}(38)-\mathrm{C}(37)-\mathrm{B}(2)$ & $112.1(14)$ & $\mathrm{C}(41)-\mathrm{C}(43)-\mathrm{H}(43 \mathrm{~A})$ & 109.5 \\
\hline $\mathrm{C}(38)-\mathrm{C}(37)-\mathrm{H}(37 \mathrm{~A})$ & 109.2 & $\mathrm{C}(41)-\mathrm{C}(43)-\mathrm{H}(43 \mathrm{~B})$ & 109.5 \\
\hline $\mathrm{B}(2)-\mathrm{C}(37)-\mathrm{H}(37 \mathrm{~A})$ & 109.2 & $\mathrm{H}(43 \mathrm{~A})-\mathrm{C}(43)-\mathrm{H}(43 \mathrm{~B})$ & 109.5 \\
\hline $\mathrm{C}(38)-\mathrm{C}(37)-\mathrm{H}(37 \mathrm{~B})$ & 109.2 & $\mathrm{C}(41)-\mathrm{C}(43)-\mathrm{H}(43 \mathrm{C})$ & 109.5 \\
\hline $\mathrm{B}(2)-\mathrm{C}(37)-\mathrm{H}(37 \mathrm{~B})$ & 109.2 & $\mathrm{H}(43 \mathrm{~A})-\mathrm{C}(43)-\mathrm{H}(43 \mathrm{C})$ & 109.5 \\
\hline $\mathrm{H}(37 \mathrm{~A})-\mathrm{C}(37)-\mathrm{H}(37 \mathrm{~B})$ & 107.9 & $\mathrm{H}(43 \mathrm{~B})-\mathrm{C}(43)-\mathrm{H}(43 \mathrm{C})$ & 109.5 \\
\hline $\mathrm{C}(37)-\mathrm{C}(38)-\mathrm{H}(38 \mathrm{~A})$ & 109.5 & $\mathrm{C}(46)-\mathrm{C}(44)-\mathrm{C}(45)$ & $109.3(11)$ \\
\hline $\mathrm{C}(37)-\mathrm{C}(38)-\mathrm{H}(38 \mathrm{~B})$ & 109.5 & $\mathrm{C}(46)-\mathrm{C}(44)-\mathrm{P}(3)$ & $115.9(9)$ \\
\hline $\mathrm{H}(38 \mathrm{~A})-\mathrm{C}(38)-\mathrm{H}(38 \mathrm{~B})$ & 109.5 & $\mathrm{C}(45)-\mathrm{C}(44)-\mathrm{P}(3)$ & $110.4(8)$ \\
\hline $\mathrm{C}(37)-\mathrm{C}(38)-\mathrm{H}(38 \mathrm{C})$ & 109.5 & $\mathrm{C}(46)-\mathrm{C}(44)-\mathrm{H}(44 \mathrm{~A})$ & 106.9 \\
\hline $\mathrm{H}(38 \mathrm{~A})-\mathrm{C}(38)-\mathrm{H}(38 \mathrm{C})$ & 109.5 & $\mathrm{C}(45)-\mathrm{C}(44)-\mathrm{H}(44 \mathrm{~A})$ & 106.9 \\
\hline $\mathrm{H}(38 \mathrm{~B})-\mathrm{C}(38)-\mathrm{H}(38 \mathrm{C})$ & 109.5 & $\mathrm{P}(3)-\mathrm{C}(44)-\mathrm{H}(44 \mathrm{~A})$ & 106.9 \\
\hline $\mathrm{C}(40)-\mathrm{C}(39)-\mathrm{P}(3)$ & $113.9(9)$ & $\mathrm{C}(44)-\mathrm{C}(45)-\mathrm{H}(45 \mathrm{~A})$ & 109.5 \\
\hline $\mathrm{C}(40)-\mathrm{C}(39)-\mathrm{H}(39 \mathrm{~A})$ & 108.8 & $\mathrm{C}(44)-\mathrm{C}(45)-\mathrm{H}(45 \mathrm{~B})$ & 109.5 \\
\hline P(3)-C(39)-H(39A) & 108.8 & $\mathrm{H}(45 \mathrm{~A})-\mathrm{C}(45)-\mathrm{H}(45 \mathrm{~B})$ & 109.5 \\
\hline $\mathrm{C}(40)-\mathrm{C}(39)-\mathrm{H}(39 \mathrm{~B})$ & 108.8 & $\mathrm{C}(44)-\mathrm{C}(45)-\mathrm{H}(45 \mathrm{C})$ & 109.5 \\
\hline $\mathrm{P}(3)-\mathrm{C}(39)-\mathrm{H}(39 \mathrm{~B})$ & 108.8 & $\mathrm{H}(45 \mathrm{~A})-\mathrm{C}(45)-\mathrm{H}(45 \mathrm{C})$ & 109.5 \\
\hline $\mathrm{H}(39 \mathrm{~A})-\mathrm{C}(39)-\mathrm{H}(39 \mathrm{~B})$ & 107.7 & $\mathrm{H}(45 \mathrm{~B})-\mathrm{C}(45)-\mathrm{H}(45 \mathrm{C})$ & 109.5 \\
\hline $\mathrm{C}(39)-\mathrm{C}(40)-\mathrm{P}(4)$ & $112.6(9)$ & $\mathrm{C}(44)-\mathrm{C}(46)-\mathrm{H}(46 \mathrm{~A})$ & 109.5 \\
\hline $\mathrm{C}(39)-\mathrm{C}(40)-\mathrm{H}(40 \mathrm{~A})$ & 109.1 & $\mathrm{C}(44)-\mathrm{C}(46)-\mathrm{H}(46 \mathrm{~B})$ & 109.5 \\
\hline
\end{tabular}




$\begin{array}{llll}\mathrm{H}(46 \mathrm{~A})-\mathrm{C}(46)-\mathrm{H}(46 \mathrm{~B}) & 109.5 & \mathrm{H}(49 \mathrm{~A})-\mathrm{C}(49)-\mathrm{H}(49 \mathrm{C}) & 109.5 \\ \mathrm{C}(44)-\mathrm{C}(46)-\mathrm{H}(46 \mathrm{C}) & 109.5 & \mathrm{H}(49 \mathrm{~B})-\mathrm{C}(49)-\mathrm{H}(49 \mathrm{C}) & 109.5 \\ \mathrm{H}(46 \mathrm{~A})-\mathrm{C}(46)-\mathrm{H}(46 \mathrm{C}) & 109.5 & \mathrm{C}(52)-\mathrm{C}(50)-\mathrm{C}(51) & 112.8(13) \\ \mathrm{H}(46 \mathrm{~B})-\mathrm{C}(46)-\mathrm{H}(46 \mathrm{C}) & 109.5 & \mathrm{C}(52)-\mathrm{C}(50)-\mathrm{P}(4) & 111.6(9) \\ \mathrm{C}(49)-\mathrm{C}(47)-\mathrm{C}(48) & 111.3(13) & \mathrm{C}(51)-\mathrm{C}(50)-\mathrm{P}(4) & 109.1(9) \\ \mathrm{C}(49)-\mathrm{C}(47)-\mathrm{P}(4) & 114.7(10) & \mathrm{C}(52)-\mathrm{C}(50)-\mathrm{H}(50 \mathrm{~A}) & 107.7 \\ \mathrm{C}(48)-\mathrm{C}(47)-\mathrm{P}(4) & 109.0(10) & \mathrm{C}(51)-\mathrm{C}(50)-\mathrm{H}(50 \mathrm{~A}) & 107.7 \\ \mathrm{C}(49)-\mathrm{C}(47)-\mathrm{H}(47 \mathrm{~A}) & 107.2 & \mathrm{P}(4)-\mathrm{C}(50)-\mathrm{H}(50 \mathrm{~A}) & 107.7 \\ \mathrm{C}(48)-\mathrm{C}(47)-\mathrm{H}(47 \mathrm{~A}) & 107.2 & \mathrm{C}(50)-\mathrm{C}(51)-\mathrm{H}(51 \mathrm{~A}) & 109.5 \\ \mathrm{P}(4)-\mathrm{C}(47)-\mathrm{H}(47 \mathrm{~A}) & 107.2 & \mathrm{C}(50)-\mathrm{C}(51)-\mathrm{H}(51 \mathrm{~B}) & 109.5 \\ \mathrm{C}(47)-\mathrm{C}(48)-\mathrm{H}(48 \mathrm{~A}) & 109.5 & \mathrm{H}(51 \mathrm{~A})-\mathrm{C}(51)-\mathrm{H}(51 \mathrm{~B}) & 109.5 \\ \mathrm{C}(47)-\mathrm{C}(48)-\mathrm{H}(48 \mathrm{~B}) & 109.5 & \mathrm{C}(50)-\mathrm{C}(51)-\mathrm{H}(51 \mathrm{C}) & 109.5 \\ \mathrm{H}(48 \mathrm{~A})-\mathrm{C}(48)-\mathrm{H}(48 \mathrm{~B}) & 109.5 & \mathrm{H}(51 \mathrm{~A})-\mathrm{C}(51)-\mathrm{H}(51 \mathrm{C}) & 109.5 \\ \mathrm{C}(47)-\mathrm{C}(48)-\mathrm{H}(48 \mathrm{C}) & 109.5 & \mathrm{H}(51 \mathrm{~B})-\mathrm{C}(51)-\mathrm{H}(51 \mathrm{C}) & 109.5 \\ \mathrm{H}(48 \mathrm{~A})-\mathrm{C}(48)-\mathrm{H}(48 \mathrm{C}) & 109.5 & \mathrm{C}(50)-\mathrm{C}(52)-\mathrm{H}(52 \mathrm{~A}) & 109.5 \\ \mathrm{H}(48 \mathrm{~B})-\mathrm{C}(48)-\mathrm{H}(48 \mathrm{C}) & 109.5 & \mathrm{C}(50)-\mathrm{C}(52)-\mathrm{H}(52 \mathrm{~B}) & 109.5 \\ \mathrm{C}(47)-\mathrm{C}(49)-\mathrm{H}(49 \mathrm{~A}) & 109.5 & \mathrm{H}(52 \mathrm{~A})-\mathrm{C}(52)-\mathrm{H}(52 \mathrm{~B}) & 109.5 \\ \mathrm{C}(47)-\mathrm{C}(49)-\mathrm{H}(49 \mathrm{~B}) & 109.5 & \mathrm{C}(50)-\mathrm{C}(52)-\mathrm{H}(52 \mathrm{C}) & 109.5 \\ \mathrm{H}(49 \mathrm{~A})-\mathrm{C}(49)-\mathrm{H}(49 \mathrm{~B}) & 109.5 & \mathrm{H}(52 \mathrm{~A})-\mathrm{C}(52)-\mathrm{H}(52 \mathrm{C}) & 109.5 \\ \mathrm{C}(47)-\mathrm{C}(49)-\mathrm{H}(49 \mathrm{C}) & 109.5 & \mathrm{H}(52 \mathrm{~B})-\mathrm{C}(52)-\mathrm{H}(52 \mathrm{C}) & 109.5 \\ & & & \end{array}$


Table S4. Anisotropic displacement parameters $\left(\AA^{2} \times 10^{3}\right)$ for jonlm07 (2a). The anisotropic displacement factor exponent takes the form: $-2 \pi^{2}\left[h^{2} a^{* 2} U_{11}+\ldots+2 h k a^{*} b^{*} U_{12}\right]$

\begin{tabular}{|c|c|c|c|c|c|c|}
\hline & $\mathrm{U}_{11}$ & $\mathrm{U}_{22}$ & $\mathrm{U}_{33}$ & $\mathrm{U}_{23}$ & $\mathrm{U}_{13}$ & $\mathrm{U}_{12}$ \\
\hline - & & & & & & \\
\hline $\mathrm{Pd} 1$ & $13(1)$ & $31(1)$ & $11(1)$ & $1(1)$ & $1(1)$ & $-4(1)$ \\
\hline $\mathrm{P} 1$ & $11(1)$ & $47(2)$ & $12(1)$ & 11(1) & $1(1)$ & $0(1)$ \\
\hline $\mathrm{P} 2$ & $15(1)$ & $22(1)$ & $15(1)$ & $-1(1)$ & $0(1)$ & $-2(1)$ \\
\hline N1 & $16(2)$ & $34(3)$ & $16(2)$ & $7(3)$ & $3(2)$ & $10(3)$ \\
\hline B1 & $20(3)$ & $39(6)$ & $13(3)$ & $9(5)$ & $-1(2)$ & $-10(5)$ \\
\hline $\mathrm{C} 1$ & $22(3)$ & $80(8)$ & $14(3)$ & $-3(6)$ & $5(2)$ & $19(6)$ \\
\hline $\mathrm{C} 2$ & $22(4)$ & $133(15)$ & $30(5)$ & $43(9)$ & $4(3)$ & $-8(8)$ \\
\hline C3 & $26(5)$ & $36(6)$ & $33(5)$ & $0(5)$ & $6(4)$ & $4(4)$ \\
\hline $\mathrm{C} 4$ & $57(9)$ & $30(7)$ & $96(13)$ & $-7(8)$ & $15(9)$ & $5(6)$ \\
\hline $\mathrm{C} 5$ & $24(4)$ & $29(5)$ & $30(5)$ & $2(4)$ & $-4(4)$ & $0(4)$ \\
\hline C6 & $42(7)$ & $45(8)$ & $89(12)$ & $-3(8)$ & $5(8)$ & $-5(6)$ \\
\hline $\mathrm{C} 7$ & $17(3)$ & $38(4)$ & $17(2)$ & $4(3)$ & $3(2)$ & $-2(3)$ \\
\hline $\mathrm{C} 8$ & $30(4)$ & $112(12)$ & $17(3)$ & $6(5)$ & $1(3)$ & $-6(6)$ \\
\hline C9 & $16(2)$ & $44(6)$ & $12(2)$ & $8(4)$ & $2(2)$ & $6(4)$ \\
\hline $\mathrm{C} 10$ & $17(3)$ & $42(6)$ & $18(3)$ & $-6(4)$ & $3(2)$ & $-7(4)$ \\
\hline $\mathrm{C} 11$ & $18(3)$ & $30(4)$ & $27(4)$ & $-5(4)$ & $-1(3)$ & $2(4)$ \\
\hline $\mathrm{C} 12$ & $23(4)$ & $18(4)$ & $67(7)$ & $7(5)$ & $4(4)$ & $-3(4)$ \\
\hline C13 & $16(3)$ & $44(6)$ & $69(8)$ & $6(8)$ & $8(4)$ & $-3(5)$ \\
\hline C14 & $18(3)$ & $26(4)$ & $29(4)$ & $-8(4)$ & $1(3)$ & $-3(4)$ \\
\hline $\mathrm{C} 15$ & $39(5)$ & $30(5)$ & $35(6)$ & $2(5)$ & $-5(4)$ & $-11(5)$ \\
\hline $\mathrm{C} 16$ & $31(4)$ & $25(5)$ & $30(4)$ & $-1(4)$ & $-10(3)$ & $2(4)$ \\
\hline $\mathrm{C} 17$ & $28(4)$ & $25(4)$ & $39(5)$ & $-7(4)$ & $-13(4)$ & $12(4)$ \\
\hline C18 & $38(6)$ & $39(6)$ & $54(8)$ & $-1(6)$ & $-10(5)$ & $15(5)$ \\
\hline C19 & $27(5)$ & $38(7)$ & $75(10)$ & $-12(6)$ & $-4(5)$ & $5(5)$ \\
\hline $\mathrm{C} 20$ & $20(2)$ & $53(5)$ & $16(2)$ & $-8(3)$ & $0(2)$ & $0(3)$ \\
\hline $\mathrm{C} 21$ & $45(6)$ & $20(5)$ & $74(9)$ & $3(6)$ & $-33(6)$ & $-9(5)$ \\
\hline $\mathrm{C} 22$ & $20(2)$ & $53(5)$ & $16(2)$ & $-8(3)$ & $0(2)$ & $0(3)$ \\
\hline $\operatorname{Pd} 2$ & $13(1)$ & $31(1)$ & $11(1)$ & $1(1)$ & $1(1)$ & $-4(1)$ \\
\hline
\end{tabular}




$\begin{array}{lcccccc}\text { P3 } & 11(1) & 47(2) & 12(1) & 11(1) & 1(1) & 0(1) \\ \text { P4 } & 15(1) & 22(1) & 15(1) & -1(1) & 0(1) & -2(1) \\ \text { N2 } & 16(2) & 34(3) & 16(2) & 7(3) & 3(2) & 10(3) \\ \text { B2 } & 20(3) & 39(6) & 13(3) & 9(5) & -1(2) & -10(5) \\ \text { C31 } & 22(3) & 80(8) & 14(3) & -3(6) & 5(2) & 19(6) \\ \text { C32 } & 22(4) & 133(15) & 30(5) & 43(9) & 4(3) & -8(8) \\ \text { C33 } & 26(5) & 36(6) & 33(5) & 0(5) & 6(4) & 4(4) \\ \text { C34 } & 57(9) & 30(7) & 96(13) & -7(8) & 15(9) & 5(6) \\ \text { C35 } & 24(4) & 29(5) & 30(5) & 2(4) & -4(4) & 0(4) \\ \text { C36 } & 42(7) & 45(8) & 89(12) & -3(8) & 5(8) & -5(6) \\ \text { C37 } & 17(3) & 38(4) & 17(2) & 4(3) & 3(2) & -2(3) \\ \text { C38 } & 30(4) & 112(12) & 17(3) & 6(5) & 1(3) & -6(6) \\ \text { C39 } & 16(2) & 44(6) & 12(2) & 8(4) & 2(2) & 6(4) \\ \text { C40 } & 17(3) & 42(6) & 18(3) & -6(4) & 3(2) & -7(4) \\ \text { C41 } & 18(3) & 30(4) & 27(4) & -5(4) & -1(3) & 2(4) \\ \text { C42 } & 23(4) & 18(4) & 67(7) & 7(5) & 4(4) & -3(4) \\ \text { C43 } & 16(3) & 44(6) & 69(8) & 6(8) & 8(4) & -3(5) \\ \text { C44 } & 18(3) & 26(4) & 29(4) & -8(4) & 1(3) & -3(4) \\ \text { C45 } & 39(5) & 30(5) & 35(6) & 2(5) & -5(4) & -11(5) \\ \text { C46 } & 31(4) & 25(5) & 30(4) & -1(4) & -10(3) & 2(4) \\ \text { C47 } & 28(4) & 25(4) & 39(5) & -7(4) & -13(4) & 12(4) \\ \text { C48 } & 38(6) & 39(6) & 54(8) & -1(6) & -10(5) & 15(5) \\ \text { C49 } & 27(5) & 38(7) & 75(10) & -12(6) & -4(5) & 5(5) \\ \text { C50 } & 20(2) & 53(5) & 16(2) & -8(3) & 0(2) & 0(3) \\ \text { C51 } & 45(6) & 20(5) & 74(9) & 3(6) & -33(6) & -9(5) \\ \text { C52 } & 20(2) & 53(5) & 16(2) & -8(3) & 0(2) & 0(3)\end{array}$


Table S5. Hydrogen coordinates (x $\left.10^{4}\right)$ and isotropic displacement parameters $\left(\AA^{2} \times 10^{3}\right)$ for jonlm07 (2a).

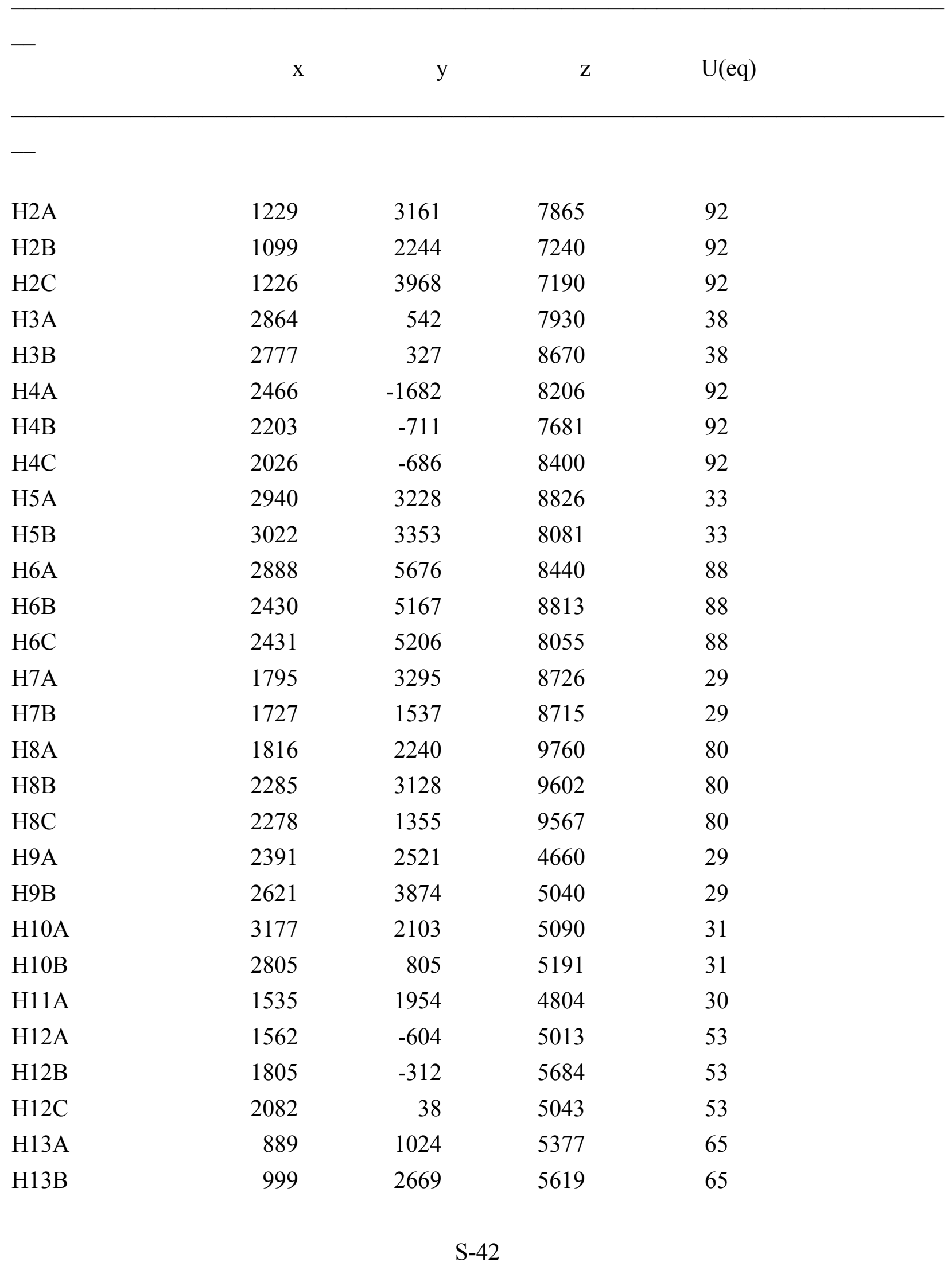




\begin{tabular}{|c|c|c|c|c|}
\hline $\mathrm{H} 13 \mathrm{C}$ & 1158 & 1271 & 6033 & 65 \\
\hline H14A & 1436 & 4653 & 5819 & 29 \\
\hline $\mathrm{H} 15 \mathrm{~A}$ & 1854 & 6807 & 5949 & 52 \\
\hline H15B & 2317 & 6066 & 5668 & 52 \\
\hline $\mathrm{H} 15 \mathrm{C}$ & 2116 & 5533 & 6338 & 52 \\
\hline H16A & 1426 & 6129 & 4936 & 43 \\
\hline H16B & 1418 & 4438 & 4707 & 43 \\
\hline H16C & 1890 & 5377 & 4666 & 43 \\
\hline H17A & 3356 & 41 & 6773 & 37 \\
\hline H18A & 2984 & -2145 & 6367 & 66 \\
\hline H18B & 2672 & -1101 & 5923 & 66 \\
\hline $\mathrm{H} 18 \mathrm{C}$ & 2629 & -983 & 6677 & 66 \\
\hline H19A & 3811 & -1244 & 6122 & 70 \\
\hline H19B & 3834 & 379 & 5817 & 70 \\
\hline H19C & 3506 & -863 & 5509 & 70 \\
\hline $\mathrm{H} 20 \mathrm{~A}$ & 3739 & 2809 & 5924 & 36 \\
\hline $\mathrm{H} 21 \mathrm{~A}$ & 3636 & 5348 & 6141 & 70 \\
\hline $\mathrm{H} 21 \mathrm{~B}$ & 3159 & 5031 & 6509 & 70 \\
\hline $\mathrm{H} 21 \mathrm{C}$ & 3186 & 4805 & 5759 & 70 \\
\hline $\mathrm{H} 22 \mathrm{~A}$ & 4037 & 3464 & 6906 & 45 \\
\hline $\mathrm{H} 22 \mathrm{~B}$ & 3784 & 1900 & 7009 & 45 \\
\hline $\mathrm{H} 22 \mathrm{C}$ & 3533 & 3398 & 7230 & 45 \\
\hline $\mathrm{H} 32 \mathrm{~A}$ & 1355 & 7409 & 6817 & 92 \\
\hline H32B & 1383 & 8254 & 7483 & 92 \\
\hline $\mathrm{H} 32 \mathrm{C}$ & 1214 & 9122 & 6863 & 92 \\
\hline H33A & -442 & 8515 & 5795 & 38 \\
\hline H33B & -480 & 8477 & 6550 & 38 \\
\hline H34A & -358 & 10867 & 6289 & 92 \\
\hline H34B & 62 & 10452 & 5823 & 92 \\
\hline $\mathrm{H} 34 \mathrm{C}$ & 128 & 10301 & 6573 & 92 \\
\hline H35A & -310 & 5603 & 6539 & 33 \\
\hline H35B & -271 & 5639 & 5785 & 33 \\
\hline H36A & 95 & 3564 & 6355 & 88 \\
\hline H36B & 509 & 4712 & 6491 & 88 \\
\hline $\mathrm{H} 36 \mathrm{C}$ & 377 & 4283 & 5778 & 88 \\
\hline H37A & 734 & 8565 & 5817 & \\
\hline
\end{tabular}




\begin{tabular}{|c|c|c|c|c|}
\hline H37B & 790 & 6806 & 5749 & 29 \\
\hline H38A & 647 & 8061 & 4759 & 80 \\
\hline H38B & 134 & 8432 & 5011 & 80 \\
\hline H38C & 287 & 6746 & 4898 & 80 \\
\hline H39A & 110 & 7248 & 9827 & 29 \\
\hline H39B & -139 & 8546 & 9438 & 29 \\
\hline $\mathrm{H} 40 \mathrm{~A}$ & -653 & 6693 & 9417 & 31 \\
\hline H40B & -260 & 5469 & 9290 & 31 \\
\hline H41A & 1005 & 6702 & 9655 & 30 \\
\hline $\mathrm{H} 42 \mathrm{~A}$ & 1018 & 4120 & 9319 & 53 \\
\hline $\mathrm{H} 42 \mathrm{~B}$ & 638 & 4549 & 8794 & 53 \\
\hline $\mathrm{H} 42 \mathrm{C}$ & 514 & 4704 & 9531 & 53 \\
\hline $\mathrm{H} 43 \mathrm{~A}$ & 1637 & 5867 & 9028 & 65 \\
\hline H43B & 1511 & 7543 & 8845 & 65 \\
\hline $\mathrm{H} 43 \mathrm{C}$ & 1348 & 6211 & 8396 & 65 \\
\hline $\mathrm{H} 44 \mathrm{~A}$ & 1077 & 9528 & 8759 & 29 \\
\hline $\mathrm{H} 45 \mathrm{~A}$ & 586 & 11695 & 8841 & 52 \\
\hline H45B & 146 & 10604 & 8877 & 52 \\
\hline $\mathrm{H} 45 \mathrm{C}$ & 464 & 10640 & 8255 & 52 \\
\hline H46A & 1004 & 10909 & 9709 & 43 \\
\hline H46B & 1131 & 9209 & 9842 & 43 \\
\hline $\mathrm{H} 46 \mathrm{C}$ & 609 & 9784 & 9944 & 43 \\
\hline H47A & -801 & 4902 & 7622 & 37 \\
\hline H48A & -329 & 2930 & 7725 & 66 \\
\hline H48B & -196 & 3318 & 8443 & 66 \\
\hline $\mathrm{H} 48 \mathrm{C}$ & 15 & 4282 & 7875 & 66 \\
\hline H49A & -1092 & 3066 & 8378 & 70 \\
\hline H49B & -1361 & 4620 & 8359 & 70 \\
\hline $\mathrm{H} 49 \mathrm{C}$ & -995 & 4285 & 8913 & 70 \\
\hline H50A & -1219 & 7403 & 8624 & 36 \\
\hline H51A & -1158 & 9983 & 8443 & 70 \\
\hline H51B & -659 & 9752 & 8118 & 70 \\
\hline $\mathrm{H} 51 \mathrm{C}$ & -725 & 9420 & 8857 & 70 \\
\hline H52A & -1504 & 8335 & 7621 & 45 \\
\hline H52B & -1386 & 6601 & 7615 & 45 \\
\hline $\mathrm{H} 52 \mathrm{C}$ & -1030 & 7759 & 7304 & 15 \\
\hline
\end{tabular}


Table S6. Torsion angles $\left[{ }^{\circ}\right]$ for jonlm07 (2a).

\begin{tabular}{|c|c|c|c|}
\hline $\mathrm{C} 1-\mathrm{N} 1-\mathrm{B} 1-\mathrm{C} 5$ & $120(3)$ & Pd1_P1_C14_C16 & $1717(0)$ \\
\hline Pd1-N1-B1-C5 & $62(2)$ & C11-P1-C14-C15 & $177.3(10)$ \\
\hline C1-N1-B1-C7 & $0(3)$ & C9-P1-C14-C15 & $-75.1(12)$ \\
\hline Pd1-N1-B1-C7 & $-177.5(13)$ & Pd1-P1-C14-C15 & $43.6(11)$ \\
\hline C1-N1-B1-C3 & $121(3)$ & C20-P2-C17-C19 & $-56.3(13)$ \\
\hline Pd1-N1-B1-C3 & $-56.9(19)$ & C10-P2-C17-C19 & $50.9(13)$ \\
\hline $\mathrm{B} 1-\mathrm{N} 1-\mathrm{C} 1-\mathrm{C} 2$ & $7(5)$ & Pd1-P2-C17-C19 & $164.6(10)$ \\
\hline Pd1-N1-C1-C2 & $-174(4)$ & C20-P2-C17-C18 & $177.0(11)$ \\
\hline B1-N1-C1-Pd1 & $-179(2)$ & C10-P2-C17-C18 & $-75.8(12)$ \\
\hline N1-B1-C3-C4 & $-59.0(19)$ & Pd1-P2-C17-C18 & $38.0(13)$ \\
\hline C5-B1-C3-C4 & $-177.4(17)$ & C17-P2-C20-C22 & $-58.6(11)$ \\
\hline C7-B1-C3-C4 & $55(2)$ & C10-P2-C20-C22 & $-164.4(10)$ \\
\hline N1-B1-C5-C6 & $62.7(19)$ & Pd1-P2-C20-C22 & $76.8(10)$ \\
\hline C7-B1-C5-C6 & $-53(2)$ & C17-P2-C20-C21 & $-178.7(12)$ \\
\hline C3-B1-C5-C6 & 177.3(17) & C10-P2-C20-C21 & $75.5(13)$ \\
\hline N1-B1-C7-C8 & $-176.3(15)$ & Pd1-P2-C20-C21 & $-43.3(13)$ \\
\hline C5-B1-C7-C8 & $-57.1(19)$ & C31-N2-B2-C33 & $-125(3)$ \\
\hline C3-B1-C7-C8 & $70.3(19)$ & Pd2-N2-B2-C33 & $62.5(17)$ \\
\hline C11-P1-C9-C10 & $-101.4(11)$ & C31-N2-B2-C37 & $-3(3)$ \\
\hline C14-P1-C9-C10 & 149.9(10) & Pd2-N2-B2-C37 & $-175.8(12)$ \\
\hline Pd1-P1-C9-C10 & $30.7(13)$ & C31-N2-B2-C35 & $117(3)$ \\
\hline P1-C9-C10-P2 & $-40.0(15)$ & $\mathrm{Pd} 2-\mathrm{N} 2-\mathrm{B} 2-\mathrm{C} 35$ & $-55.2(18)$ \\
\hline C20-P2-C10-C9 & $-100.4(11)$ & B2-N2-C31-C32 & $11(5)$ \\
\hline C17-P2-C10-C9 & $152.9(11)$ & $\mathrm{Pd} 2-\mathrm{N} 2-\mathrm{C} 31-\mathrm{C} 32$ & $-174(3)$ \\
\hline Pd1-P2-C10-C9 & $30.5(11)$ & B2-N2-C31-Pd2 & $-175(2)$ \\
\hline C14-P1-C11-C12 & $177.5(10)$ & N2-B2-C33-C34 & $65.3(19)$ \\
\hline C9-P1-C11-C12 & $67.7(12)$ & C37-B2-C33-C34 & $-51(2)$ \\
\hline Pd1-P1-C11-C12 & $-56.1(11)$ & C35-B2-C33-C34 & $180.0(17)$ \\
\hline C14-P1-C11-C13 & $-62.0(12)$ & N2-B2-C35-C36 & $-66.2(19)$ \\
\hline C9-P1-C11-C13 & $-171.8(11)$ & C33-B2-C35-C36 & $-179.9(17)$ \\
\hline Pd1-P1-C11-C13 & $64.4(12)$ & $\mathrm{C} 37-\mathrm{B} 2-\mathrm{C} 35-\mathrm{C} 36$ & $50(2)$ \\
\hline C11-P1-C14-C16 & $-54.6(11)$ & N2-B2-C37-C38 & $-172.2(15)$ \\
\hline C9-P1-C14-C16 & $53.0(12)$ & C33-B2-C37-C38 & $-56.9(19)$ \\
\hline
\end{tabular}




\begin{tabular}{lc} 
C35-B2-C37-C38 & $71.4(18)$ \\
C41-P3-C39-C40 & $-95.6(11)$ \\
C44-P3-C39-C40 & $155.4(10)$ \\
Pd2-P3-C39-C40 & $35.6(11)$ \\
P3-C39-C40-P4 & $-42.8(14)$ \\
C47-P4-C40-C39 & $152.2(10)$ \\
C50-P4-C40-C39 & $-99.4(11)$ \\
Pd2-P4-C40-C39 & $28.9(11)$ \\
C44-P3-C41-C42 & $173.2(11)$ \\
C39-P3-C41-C42 & $62.3(12)$ \\
Pd2-P3-C41-C42 & $-57.6(12)$ \\
C44-P3-C41-C43 & $-63.7(12)$ \\
C39-P3-C41-C43 & $-174.6(11)$ \\
Pd2-P3-C41-C43 & $65.4(12)$ \\
C41-P3-C44-C46 & $-56.7(11)$ \\
C39-P3-C44-C46 & $53.2(11)$ \\
Pd2-P3-C44-C46 & $168.1(9)$ \\
C41-P3-C44-C45 & $178.4(10)$ \\
C39-P3-C44-C45 & $-71.7(11)$ \\
Pd2-P3-C44-C45 & $43.2(11)$ \\
C50-P4-C47-C49 & $-58.3(12)$ \\
C40-P4-C47-C49 & $49.3(12)$ \\
Pd2-P4-C47-C49 & $166.2(10)$ \\
C50-P4-C47-C48 & $176.1(11)$ \\
C40-P4-C47-C48 & $-76.3(12)$ \\
Pd2-P4-C47-C48 & $40.6(13)$ \\
C47-P4-C50-C52 & $-50.1(12)$ \\
C40-P4-C50-C52 & $-161.2(11)$ \\
Pd2-P4-C50-C52 & $81.7(11)$ \\
C47-P4-C50-C51 & $-175.4(12)$ \\
C4-P4-C50-C51 & $73.5(13)$ \\
\hline
\end{tabular}


REFERENCE NUMBER: jonlm36 (3b)

\title{
CRYSTAL STRUCTURE REPORT
}

$$
\mathrm{C}_{27} \mathrm{H}_{52} \text { B N P } 2 \text { Pd }
$$

Report prepared for:

L. Munjanja, Prof. W. Jones

February 22, 2016

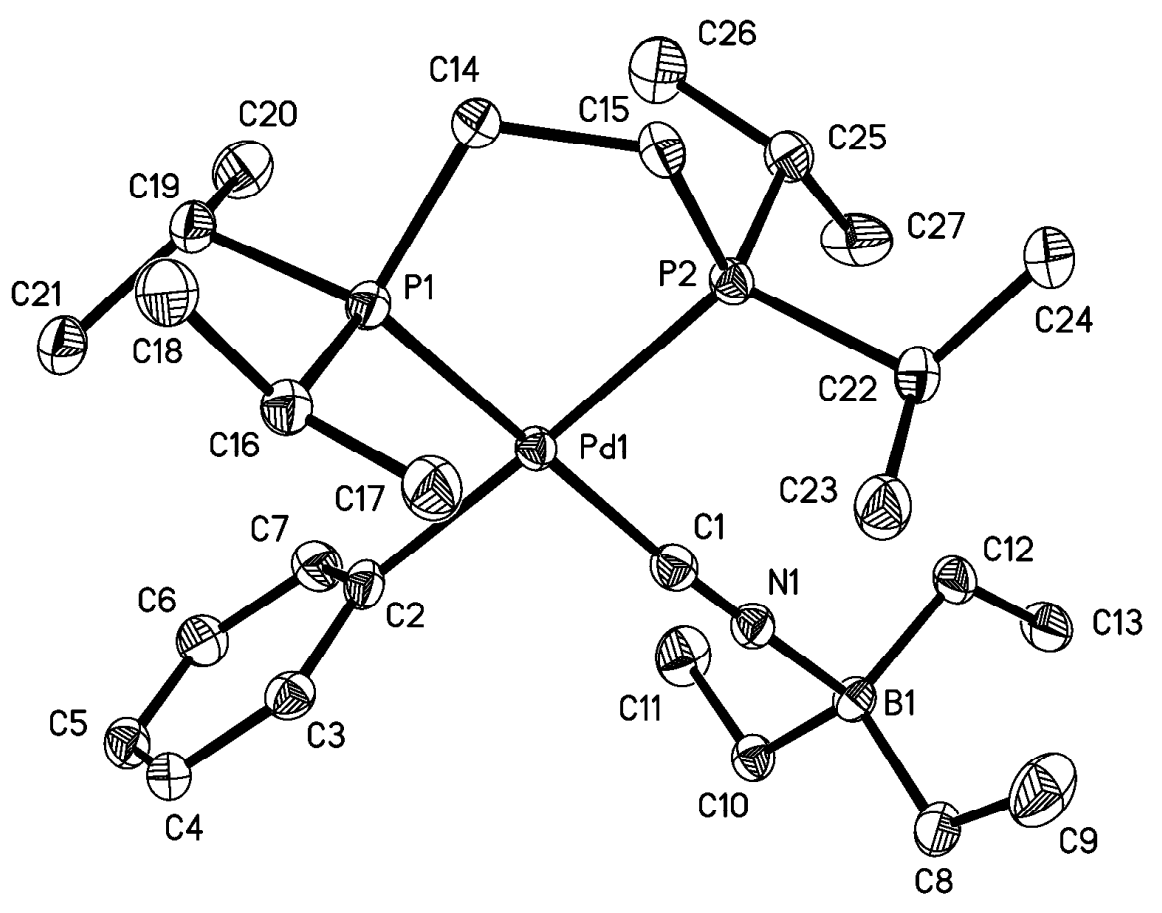

\author{
William W. Brennessel \\ X-ray Crystallographic Facility \\ Department of Chemistry, University of Rochester \\ 120 Trustee Road \\ Rochester, NY 14627
}




\section{Data collection}

A crystal $\left(0.24 \times 0.20 \times 0.18 \mathrm{~mm}^{3}\right)$ was placed onto the tip of a thin glass optical fiber and mounted on a Bruker SMART APEX II CCD platform diffractometer for a data collection at 100.0(5) K. ${ }^{1}$ A preliminary set of cell constants and an orientation matrix were calculated from reflections harvested from three orthogonal wedges of reciprocal space. The full data collection was carried out using $\mathrm{MoK} \alpha$ radiation (graphite monochromator) with a frame time of 45 seconds and a detector distance of $4.01 \mathrm{~cm}$. A randomly oriented region of reciprocal space was surveyed: six major sections of frames were collected with $0.50^{\circ}$ steps in $\omega$ at six different $\phi$ settings and a detector position of $-38^{\circ}$ in $2 \theta$. The intensity data were corrected for absorption. ${ }^{2}$ Final cell constants were calculated from the xyz centroids of 3747 strong reflections from the actual data collection after integration. ${ }^{3}$ See Table S7 for additional crystal and refinement information.

Structure solution and refinement

The structure was solved using SHELXT-2014/54 and refined using SHELXL-2014/7.5 The space group $P b c a$ was determined based on systematic absences. A direct-methods solution was calculated which provided most non-hydrogen atoms from the E-map. Full-matrix least squares / difference Fourier cycles were performed which located the remaining non-hydrogen atoms. All non-hydrogen atoms were refined with anisotropic displacement parameters. All hydrogen atoms were placed in ideal positions and refined as riding atoms with relative isotropic displacement parameters. The final full matrix least squares refinement converged to $R 1=$ $0.0410\left(F^{2}, I>2 \sigma(I)\right)$ and $w R 2=0.0999\left(F^{2}\right.$, all data $)$.

\section{Structure description}

The structure is the one suggested. The asymmetric unit contains one molecule in a general position.

Unless noted otherwise all structural diagrams containing thermal displacement ellipsoids are drawn at the $50 \%$ probability level.

Data collection, structure solution, and structure refinement were conducted at the X-ray Crystallographic Facility, B51 Hutchison Hall, Department of Chemistry, University of Rochester. All publications arising from this report MUST either 1) include William W. Brennessel as a coauthor or 2) acknowledge William W. Brennessel and the X-ray Crystallographic Facility of the Department of Chemistry at the University of Rochester. 
1 APEX3, version 2015.9-0; Bruker AXS: Madison, WI, 2015.

2 Sheldrick, G. M. SADABS, version 2014/5; J. Appl. Cryst. 2015, 48, 3-10.

3 SAINT, version 8.34A; Bruker AXS: Madison, WI, 2013.

4 Sheldrick, G. M. SHELXT-2014/5; University of Göttingen: Göttingen, Germany, 2014.

5 Sheldrick, G. M. SHELXL-2014/7; Acta. Cryst. 2015, C71, 3-8.

Some equations of interest:

$$
\begin{gathered}
R_{\text {int }}=\Sigma\left|F_{\mathrm{O}}^{2}-<F_{\mathrm{O}}^{2}>\right| / \Sigma\left|F_{\mathrm{O}}{ }^{2}\right| \\
R 1=\Sigma|| F_{\mathrm{O}}|-| F_{\mathrm{c}} \| / \Sigma\left|F_{\mathrm{O}}\right| \\
w R 2=\left[\Sigma\left[w\left(F_{\mathrm{O}}^{2}-F_{\mathrm{c}}{ }^{2}\right)^{2}\right] / \Sigma\left[w\left(F_{\mathrm{O}}^{2}\right)^{2}\right]\right]^{1 / 2} \\
\text { where } w=1 /\left[\sigma^{2}\left(F_{\mathrm{O}}^{2}\right)+(a P)^{2}+b P\right] \text { and } \\
P=1 / 3 \max \left(0, F_{\mathrm{O}}^{2}\right)+2 / 3 F_{\mathrm{c}}^{2} \\
\text { GOF }=S=\left[\Sigma\left[w\left(F_{\mathrm{O}}^{2}-F_{\mathrm{c}}^{2}\right)^{2}\right] /(m-n)\right]^{1 / 2}
\end{gathered}
$$

where $m=$ number of reflections and $n=$ number of parameters 


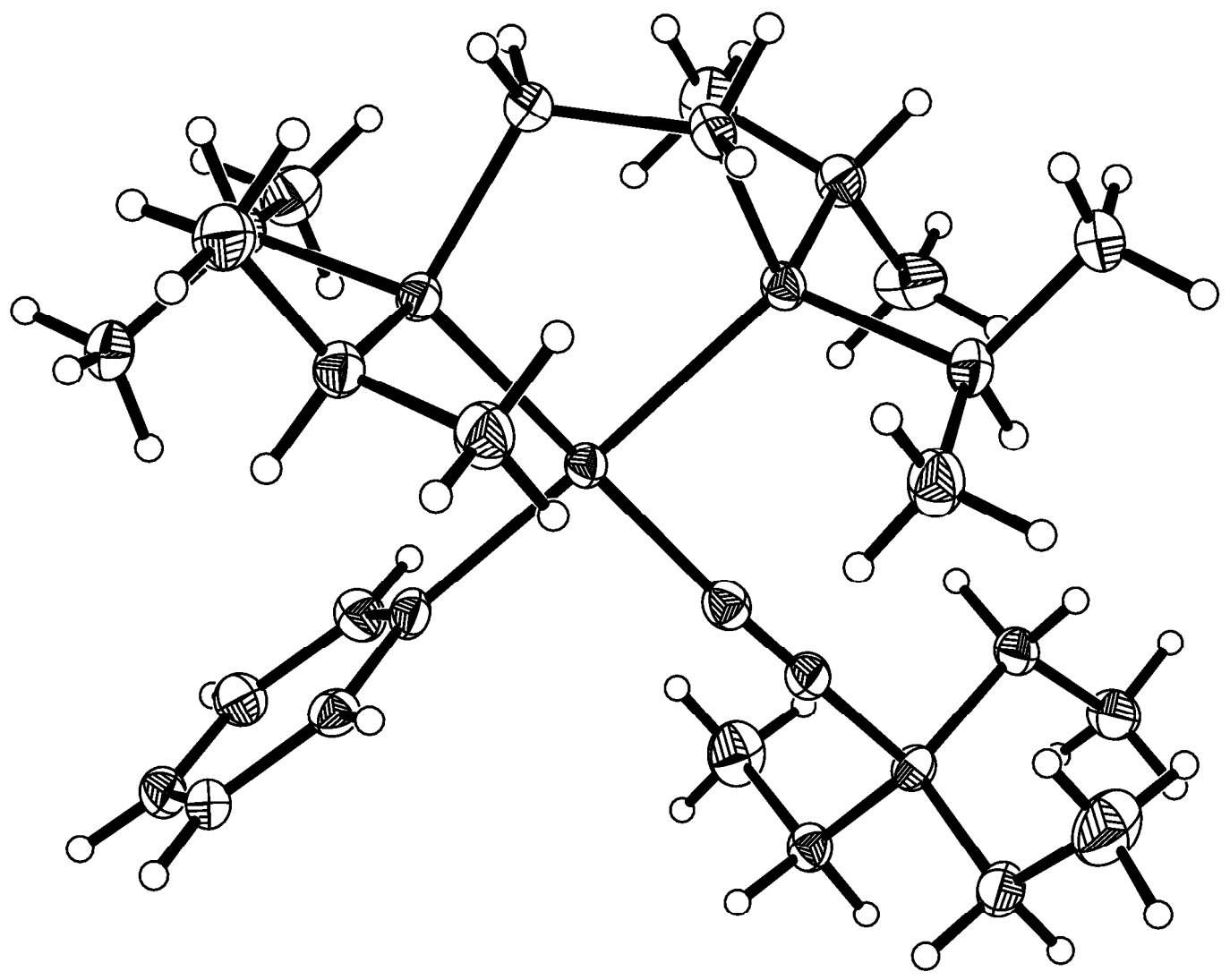


Table S7. Crystal data and structure refinement for jonlm36 (3b).

\begin{tabular}{|c|c|c|}
\hline Identification code & \multicolumn{2}{|l|}{ jonlm36 } \\
\hline Empirical formula & \multicolumn{2}{|l|}{ C27 H52 B N P2 Pd } \\
\hline Formula weight & \multicolumn{2}{|l|}{569.84} \\
\hline Temperature & \multicolumn{2}{|l|}{$100.0(5) \mathrm{K}$} \\
\hline Wavelength & \multicolumn{2}{|l|}{$0.71073 \AA$} \\
\hline Crystal system & \multicolumn{2}{|l|}{ orthorhombic } \\
\hline Space group & \multicolumn{2}{|l|}{ Pbca } \\
\hline \multirow[t]{3}{*}{ Unit cell dimensions } & $a=15.9698(13) \AA$ & $\alpha=90^{\circ}$ \\
\hline & $b=17.7847(15) \AA$ & $\beta=90^{\circ}$ \\
\hline & $c=21.9529(19) \AA$ & $\gamma=90^{\circ}$ \\
\hline Volume & \multicolumn{2}{|l|}{$6235.0(9) \AA^{3}$} \\
\hline$Z$ & \multicolumn{2}{|l|}{8} \\
\hline Density (calculated) & \multicolumn{2}{|l|}{$1.214 \mathrm{Mg} / \mathrm{m}^{3}$} \\
\hline Absorption coefficient & \multicolumn{2}{|l|}{$0.712 \mathrm{~mm}^{-1}$} \\
\hline$F(000)$ & \multicolumn{2}{|l|}{2416} \\
\hline Crystal color, morphology & \multicolumn{2}{|l|}{ pale yellow, block } \\
\hline Crystal size & \multicolumn{2}{|c|}{$0.24 \times 0.20 \times 0.18 \mathrm{~mm}^{3}$} \\
\hline Theta range for data collection & \multicolumn{2}{|l|}{1.855 to $27.516^{\circ}$} \\
\hline Index ranges & \multicolumn{2}{|c|}{$-20 \leq h \leq 20,-23 \leq k \leq 23,-28 \leq l \leq 28$} \\
\hline Reflections collected & \multicolumn{2}{|l|}{111470} \\
\hline Independent reflections & \multicolumn{2}{|c|}{$7165[R(\mathrm{int})=0.1245]$} \\
\hline Observed reflections & \multicolumn{2}{|l|}{5114} \\
\hline Completeness to theta $=27.485^{\circ}$ & \multicolumn{2}{|l|}{$100.0 \%$} \\
\hline Absorption correction & \multicolumn{2}{|l|}{ Multi-scan } \\
\hline Max. and min. transmission & \multicolumn{2}{|l|}{0.7456 and 0.6588} \\
\hline Refinement method & \multicolumn{2}{|c|}{ Full-matrix least-squares on $F^{2}$} \\
\hline Data / restraints / parameters & \multicolumn{2}{|l|}{$7165 / 0 / 300$} \\
\hline Goodness-of-fit on $F^{2}$ & \multicolumn{2}{|l|}{1.042} \\
\hline Final $R$ indices $[I>2 \operatorname{sigma}(I)]$ & \multicolumn{2}{|c|}{$R 1=0.0410, w R 2=0.0854$} \\
\hline$R$ indices (all data) & \multicolumn{2}{|c|}{$R 1=0.0716, w R 2=0.0999$} \\
\hline Largest diff. peak and hole & \multicolumn{2}{|c|}{0.813 and -0.802 e..$\AA^{-3}$} \\
\hline
\end{tabular}


Table S8. Atomic coordinates $\left(\mathrm{x} 10^{4}\right)$ and equivalent isotropic displacement parameters $\left(\AA^{2} \mathrm{X}\right.$ $10^{3}$ ) for jonlm36 (3b). $U_{e q}$ is defined as one third of the trace of the orthogonalized $U_{i j}$ tensor.

\begin{tabular}{|c|c|c|c|c|}
\hline 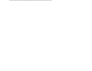 & $\mathrm{x}$ & $\mathrm{y}$ & Z & $\mathrm{U}_{\mathrm{eq}}$ \\
\hline \multicolumn{5}{|l|}{ - } \\
\hline $\operatorname{Pd} 1$ & $7142(1)$ & $5174(1)$ & $6110(1)$ & $15(1)$ \\
\hline $\mathrm{P} 1$ & $7952(1)$ & $4348(1)$ & $5570(1)$ & $16(1)$ \\
\hline $\mathrm{P} 2$ & $6852(1)$ & 4181(1) & $6762(1)$ & $16(1)$ \\
\hline N1 & $6052(2)$ & $6288(2)$ & $6900(1)$ & $19(1)$ \\
\hline B1 & $5453(2)$ & $6820(2)$ & $7292(2)$ & $18(1)$ \\
\hline $\mathrm{C} 1$ & $6453(2)$ & $5902(2)$ & $6603(2)$ & $20(1)$ \\
\hline $\mathrm{C} 2$ & $7390(2)$ & $6033(2)$ & $5503(2)$ & $18(1)$ \\
\hline $\mathrm{C} 3$ & $8169(2)$ & $6385(2)$ & $5457(2)$ & $19(1)$ \\
\hline $\mathrm{C} 4$ & $8326(2)$ & $6927(2)$ & $5011(2)$ & $20(1)$ \\
\hline $\mathrm{C} 5$ & $7703(2)$ & $7131(2)$ & $4599(2)$ & $23(1)$ \\
\hline C6 & $6921(2)$ & $6806(2)$ & $4649(2)$ & $24(1)$ \\
\hline $\mathrm{C} 7$ & $6765(2)$ & $6267(2)$ & $5102(2)$ & $24(1)$ \\
\hline $\mathrm{C} 8$ & $6037(2)$ & $7186(2)$ & $7827(2)$ & $25(1)$ \\
\hline C9 & $6430(3)$ & $6631(3)$ & $8276(2)$ & $43(1)$ \\
\hline $\mathrm{C} 10$ & $5139(2)$ & $7468(2)$ & $6825(2)$ & $21(1)$ \\
\hline $\mathrm{C} 11$ & $4554(3)$ & $7214(2)$ & $6317(2)$ & $34(1)$ \\
\hline $\mathrm{C} 12$ & $4709(2)$ & $6274(2)$ & $7542(2)$ & $24(1)$ \\
\hline $\mathrm{C} 13$ & 4033(3) & $6688(2)$ & $7907(2)$ & $31(1)$ \\
\hline $\mathrm{C} 14$ & $7781(2)$ & $3398(2)$ & $5885(2)$ & $20(1)$ \\
\hline $\mathrm{C} 15$ & $7563(2)$ & $3400(2)$ & $6568(2)$ & $20(1)$ \\
\hline $\mathrm{C} 16$ & $9086(2)$ & $4525(2)$ & $5623(2)$ & $20(1)$ \\
\hline $\mathrm{C} 17$ & $9365(2)$ & $4632(2)$ & $6285(2)$ & $26(1)$ \\
\hline $\mathrm{C} 18$ & $9620(2)$ & $3928(2)$ & $5304(2)$ & $27(1)$ \\
\hline C19 & $7749(2)$ & $4224(2)$ & $4746(2)$ & $21(1)$ \\
\hline $\mathrm{C} 20$ & $6804(2)$ & $4170(2)$ & $4629(2)$ & $28(1)$ \\
\hline $\mathrm{C} 21$ & $8142(2)$ & $4845(2)$ & $4357(2)$ & $26(1)$ \\
\hline $\mathrm{C} 22$ & $7032(2)$ & $4383(2)$ & $7578(2)$ & $21(1)$ \\
\hline $\mathrm{C} 23$ & $7879(2)$ & $4776(2)$ & $7657(2)$ & $28(1)$ \\
\hline
\end{tabular}




$\begin{array}{lllll}\mathrm{C} 24 & 6952(2) & 3696(2) & 7992(2) & 27(1) \\ \mathrm{C} 25 & 5787(2) & 3783(2) & 6718(2) & 21(1) \\ \mathrm{C} 26 & 5588(2) & 3524(2) & 6071(2) & 32(1) \\ \mathrm{C} 27 & 5133(2) & 4335(2) & 6940(2) & 32(1)\end{array}$


Table S9. Bond lengths $[\AA]$ and angles $\left[{ }^{\circ}\right]$ for jonlm $36(3 b)$.

\begin{tabular}{|c|c|c|c|}
\hline $\operatorname{Pd}(1)-C(1)$ & $2.016(4)$ & $\mathrm{C}(10)-\mathrm{H}(10 \mathrm{~B})$ & 0.9900 \\
\hline $\operatorname{Pd}(1)-C(2)$ & $2.065(3)$ & $\mathrm{C}(11)-\mathrm{H}(11 \mathrm{~A})$ & 0.9800 \\
\hline $\mathrm{Pd}(1)-\mathrm{P}(1)$ & $2.2866(9)$ & $\mathrm{C}(11)-\mathrm{H}(11 \mathrm{~B})$ & 0.9800 \\
\hline $\mathrm{Pd}(1)-\mathrm{P}(2)$ & $2.3205(9)$ & $\mathrm{C}(11)-\mathrm{H}(11 \mathrm{C})$ & 0.9800 \\
\hline$P(1)-C(16)$ & $1.842(3)$ & $\mathrm{C}(12)-\mathrm{C}(13)$ & $1.532(5)$ \\
\hline $\mathrm{P}(1)-\mathrm{C}(14)$ & $1.847(3)$ & $\mathrm{C}(12)-\mathrm{H}(12 \mathrm{~A})$ & 0.9900 \\
\hline $\mathrm{P}(1)-\mathrm{C}(19)$ & $1.850(3)$ & $\mathrm{C}(12)-\mathrm{H}(12 \mathrm{~B})$ & 0.9900 \\
\hline $\mathrm{P}(2)-\mathrm{C}(15)$ & $1.845(3)$ & $\mathrm{C}(13)-\mathrm{H}(13 \mathrm{~A})$ & 0.9800 \\
\hline $\mathrm{P}(2)-\mathrm{C}(25)$ & $1.845(3)$ & $\mathrm{C}(13)-\mathrm{H}(13 \mathrm{~B})$ & 0.9800 \\
\hline $\mathrm{P}(2)-\mathrm{C}(22)$ & $1.850(3)$ & $\mathrm{C}(13)-\mathrm{H}(13 \mathrm{C})$ & 0.9800 \\
\hline $\mathrm{N}(1)-\mathrm{C}(1)$ & $1.143(4)$ & $\mathrm{C}(14)-\mathrm{C}(15)$ & $1.539(5)$ \\
\hline $\mathrm{N}(1)-\mathrm{B}(1)$ & $1.598(5)$ & $\mathrm{C}(14)-\mathrm{H}(14 \mathrm{~A})$ & 0.9900 \\
\hline $\mathrm{B}(1)-\mathrm{C}(10)$ & $1.623(5)$ & $\mathrm{C}(14)-\mathrm{H}(14 \mathrm{~B})$ & 0.9900 \\
\hline $\mathrm{B}(1)-\mathrm{C}(12)$ & $1.629(5)$ & $\mathrm{C}(15)-\mathrm{H}(15 \mathrm{~A})$ & 0.9900 \\
\hline $\mathrm{B}(1)-\mathrm{C}(8)$ & $1.634(5)$ & $\mathrm{C}(15)-\mathrm{H}(15 \mathrm{~B})$ & 0.9900 \\
\hline$C(2)-C(7)$ & $1.395(5)$ & $C(16)-C(17)$ & $1.531(5)$ \\
\hline$C(2)-C(3)$ & $1.396(5)$ & $C(16)-C(18)$ & $1.532(5)$ \\
\hline$C(3)-C(4)$ & $1.397(5)$ & $\mathrm{C}(16)-\mathrm{H}(16 \mathrm{~A})$ & 1.0000 \\
\hline $\mathrm{C}(3)-\mathrm{H}(3 \mathrm{~A})$ & 0.9500 & $\mathrm{C}(17)-\mathrm{H}(17 \mathrm{~A})$ & 0.9800 \\
\hline$C(4)-C(5)$ & $1.393(5)$ & $\mathrm{C}(17)-\mathrm{H}(17 \mathrm{~B})$ & 0.9800 \\
\hline $\mathrm{C}(4)-\mathrm{H}(4 \mathrm{~A})$ & 0.9500 & $\mathrm{C}(17)-\mathrm{H}(17 \mathrm{C})$ & 0.9800 \\
\hline$C(5)-C(6)$ & $1.381(5)$ & $\mathrm{C}(18)-\mathrm{H}(18 \mathrm{~A})$ & 0.9800 \\
\hline $\mathrm{C}(5)-\mathrm{H}(5 \mathrm{~A})$ & 0.9500 & $\mathrm{C}(18)-\mathrm{H}(18 \mathrm{~B})$ & 0.9800 \\
\hline$C(6)-C(7)$ & $1.403(5)$ & $\mathrm{C}(18)-\mathrm{H}(18 \mathrm{C})$ & 0.9800 \\
\hline $\mathrm{C}(6)-\mathrm{H}(6 \mathrm{~A})$ & 0.9500 & $C(19)-C(21)$ & $1.531(5)$ \\
\hline $\mathrm{C}(7)-\mathrm{H}(7 \mathrm{~A})$ & 0.9500 & $C(19)-C(20)$ & $1.535(5)$ \\
\hline$C(8)-C(9)$ & $1.528(5)$ & $\mathrm{C}(19)-\mathrm{H}(19 \mathrm{~A})$ & 1.0000 \\
\hline $\mathrm{C}(8)-\mathrm{H}(8 \mathrm{~A})$ & 0.9900 & $\mathrm{C}(20)-\mathrm{H}(20 \mathrm{~A})$ & 0.9800 \\
\hline $\mathrm{C}(8)-\mathrm{H}(8 \mathrm{~B})$ & 0.9900 & $\mathrm{C}(20)-\mathrm{H}(20 \mathrm{~B})$ & 0.9800 \\
\hline $\mathrm{C}(9)-\mathrm{H}(9 \mathrm{~A})$ & 0.9800 & $\mathrm{C}(20)-\mathrm{H}(20 \mathrm{C})$ & 0.9800 \\
\hline $\mathrm{C}(9)-\mathrm{H}(9 \mathrm{~B})$ & 0.9800 & $\mathrm{C}(21)-\mathrm{H}(21 \mathrm{~A})$ & 0.9800 \\
\hline $\mathrm{C}(9)-\mathrm{H}(9 \mathrm{C})$ & 0.9800 & $\mathrm{C}(21)-\mathrm{H}(21 \mathrm{~B})$ & 0.9800 \\
\hline $\mathrm{C}(10)-\mathrm{C}(11)$ & $1.523(5)$ & $\mathrm{C}(21)-\mathrm{H}(21 \mathrm{C})$ & 0.9800 \\
\hline $\mathrm{C}(10)-\mathrm{H}(10 \mathrm{~A})$ & 0.9900 & $C(22)-C(24)$ & $1.527(5)$ \\
\hline
\end{tabular}




\begin{tabular}{|c|c|c|c|}
\hline$C(22)-C(23)$ & $1.532(5)$ & $\mathrm{N}(1)-\mathrm{B}(1)-\mathrm{C}(10)$ & $105.4(3)$ \\
\hline $\mathrm{C}(22)-\mathrm{H}(22 \mathrm{~A})$ & 1.0000 & $\mathrm{~N}(1)-\mathrm{B}(1)-\mathrm{C}(12)$ & $105.4(3)$ \\
\hline $\mathrm{C}(23)-\mathrm{H}(23 \mathrm{~A})$ & 0.9800 & $\mathrm{C}(10)-\mathrm{B}(1)-\mathrm{C}(12)$ & $114.3(3)$ \\
\hline $\mathrm{C}(23)-\mathrm{H}(23 \mathrm{~B})$ & 0.9800 & $\mathrm{~N}(1)-\mathrm{B}(1)-\mathrm{C}(8)$ & $106.3(3)$ \\
\hline $\mathrm{C}(23)-\mathrm{H}(23 \mathrm{C})$ & 0.9800 & $\mathrm{C}(10)-\mathrm{B}(1)-\mathrm{C}(8)$ & $110.4(3)$ \\
\hline $\mathrm{C}(24)-\mathrm{H}(24 \mathrm{~A})$ & 0.9800 & $\mathrm{C}(12)-\mathrm{B}(1)-\mathrm{C}(8)$ & $114.3(3)$ \\
\hline $\mathrm{C}(24)-\mathrm{H}(24 \mathrm{~B})$ & 0.9800 & $\mathrm{~N}(1)-\mathrm{C}(1)-\mathrm{Pd}(1)$ & $176.8(3)$ \\
\hline $\mathrm{C}(24)-\mathrm{H}(24 \mathrm{C})$ & 0.9800 & $\mathrm{C}(7)-\mathrm{C}(2)-\mathrm{C}(3)$ & $117.3(3)$ \\
\hline$C(25)-C(27)$ & $1.514(5)$ & $C(7)-C(2)-P d(1)$ & $119.4(3)$ \\
\hline$C(25)-C(26)$ & $1.526(5)$ & $C(3)-C(2)-P d(1)$ & $123.3(2)$ \\
\hline $\mathrm{C}(25)-\mathrm{H}(25 \mathrm{~A})$ & 1.0000 & $C(2)-C(3)-C(4)$ & $121.3(3)$ \\
\hline $\mathrm{C}(26)-\mathrm{H}(26 \mathrm{~A})$ & 0.9800 & $\mathrm{C}(2)-\mathrm{C}(3)-\mathrm{H}(3 \mathrm{~A})$ & 119.4 \\
\hline $\mathrm{C}(26)-\mathrm{H}(26 \mathrm{~B})$ & 0.9800 & $\mathrm{C}(4)-\mathrm{C}(3)-\mathrm{H}(3 \mathrm{~A})$ & 119.4 \\
\hline$C(26)-H(26 C)$ & 0.9800 & $C(5)-C(4)-C(3)$ & $120.5(3)$ \\
\hline $\mathrm{C}(27)-\mathrm{H}(27 \mathrm{~A})$ & 0.9800 & $\mathrm{C}(5)-\mathrm{C}(4)-\mathrm{H}(4 \mathrm{~A})$ & 119.8 \\
\hline $\mathrm{C}(27)-\mathrm{H}(27 \mathrm{~B})$ & 0.9800 & $\mathrm{C}(3)-\mathrm{C}(4)-\mathrm{H}(4 \mathrm{~A})$ & 119.8 \\
\hline $\mathrm{C}(27)-\mathrm{H}(27 \mathrm{C})$ & 0.9800 & $C(6)-C(5)-C(4)$ & 119.1(3) \\
\hline $\mathrm{C}(1)-\mathrm{Pd}(1)-\mathrm{C}(2)$ & $88.64(13)$ & $\mathrm{C}(6)-\mathrm{C}(5)-\mathrm{H}(5 \mathrm{~A})$ & 120.5 \\
\hline $\mathrm{C}(1)-\mathrm{Pd}(1)-\mathrm{P}(1)$ & 178.38(10) & $\mathrm{C}(4)-\mathrm{C}(5)-\mathrm{H}(5 \mathrm{~A})$ & 120.5 \\
\hline$C(2)-P d(1)-P(1)$ & $91.87(9)$ & $C(5)-C(6)-C(7)$ & $120.2(3)$ \\
\hline $\mathrm{C}(1)-\mathrm{Pd}(1)-\mathrm{P}(2)$ & $92.72(10)$ & $\mathrm{C}(5)-\mathrm{C}(6)-\mathrm{H}(6 \mathrm{~A})$ & 119.9 \\
\hline$C(2)-P d(1)-P(2)$ & 177.97(10) & $\mathrm{C}(7)-\mathrm{C}(6)-\mathrm{H}(6 \mathrm{~A})$ & 119.9 \\
\hline$P(1)-P d(1)-P(2)$ & $86.82(3)$ & $C(2)-C(7)-C(6)$ & $121.6(3)$ \\
\hline$C(16)-P(1)-C(14)$ & $106.10(16)$ & $\mathrm{C}(2)-\mathrm{C}(7)-\mathrm{H}(7 \mathrm{~A})$ & 119.2 \\
\hline$C(16)-P(1)-C(19)$ & $104.75(16)$ & $\mathrm{C}(6)-\mathrm{C}(7)-\mathrm{H}(7 \mathrm{~A})$ & 119.2 \\
\hline$C(14)-P(1)-C(19)$ & $103.34(15)$ & $\mathrm{C}(9)-\mathrm{C}(8)-\mathrm{B}(1)$ & $116.1(3)$ \\
\hline$C(16)-P(1)-P d(1)$ & $114.42(11)$ & $\mathrm{C}(9)-\mathrm{C}(8)-\mathrm{H}(8 \mathrm{~A})$ & 108.2 \\
\hline$C(14)-P(1)-P d(1)$ & $108.10(11)$ & $\mathrm{B}(1)-\mathrm{C}(8)-\mathrm{H}(8 \mathrm{~A})$ & 108.2 \\
\hline$C(19)-P(1)-P d(1)$ & 118.93(12) & $\mathrm{C}(9)-\mathrm{C}(8)-\mathrm{H}(8 \mathrm{~B})$ & 108.2 \\
\hline $\mathrm{C}(15)-\mathrm{P}(2)-\mathrm{C}(25)$ & 105.40(16) & $\mathrm{B}(1)-\mathrm{C}(8)-\mathrm{H}(8 \mathrm{~B})$ & 108.2 \\
\hline$C(15)-P(2)-C(22)$ & 105.92(16) & $\mathrm{H}(8 \mathrm{~A})-\mathrm{C}(8)-\mathrm{H}(8 \mathrm{~B})$ & 107.4 \\
\hline$C(25)-P(2)-C(22)$ & 105.63(16) & $\mathrm{C}(8)-\mathrm{C}(9)-\mathrm{H}(9 \mathrm{~A})$ & 109.5 \\
\hline$C(15)-P(2)-P d(1)$ & 107.89(11) & $\mathrm{C}(8)-\mathrm{C}(9)-\mathrm{H}(9 \mathrm{~B})$ & 109.5 \\
\hline$C(25)-P(2)-P d(1)$ & $116.37(12)$ & $\mathrm{H}(9 \mathrm{~A})-\mathrm{C}(9)-\mathrm{H}(9 \mathrm{~B})$ & 109.5 \\
\hline $\mathrm{C}(22)-\mathrm{P}(2)-\mathrm{Pd}(1)$ & 114.79(11) & $\mathrm{C}(8)-\mathrm{C}(9)-\mathrm{H}(9 \mathrm{C})$ & 109.5 \\
\hline $\mathrm{C}(1)-\mathrm{N}(1)-\mathrm{B}(1)$ & 177.1(3) & $\mathrm{H}(9 \mathrm{~A})-\mathrm{C}(9)-\mathrm{H}(9 \mathrm{C})$ & 109.5 \\
\hline
\end{tabular}




\begin{tabular}{|c|c|c|c|}
\hline $\mathrm{H}(9 \mathrm{~B})-\mathrm{C}(9)-\mathrm{H}(9 \mathrm{C})$ & 109.5 & $\mathrm{H}(15 \mathrm{~A})-\mathrm{C}(15)-\mathrm{H}(15 \mathrm{~B})$ & 108.0 \\
\hline $\mathrm{C}(11)-\mathrm{C}(10)-\mathrm{B}(1)$ & 116.2(3) & $\mathrm{C}(17)-\mathrm{C}(16)-\mathrm{C}(18)$ & $111.0(3)$ \\
\hline $\mathrm{C}(11)-\mathrm{C}(10)-\mathrm{H}(10 \mathrm{~A})$ & 108.2 & $\mathrm{C}(17)-\mathrm{C}(16)-\mathrm{P}(1)$ & $111.6(2)$ \\
\hline $\mathrm{B}(1)-\mathrm{C}(10)-\mathrm{H}(10 \mathrm{~A})$ & 108.2 & $\mathrm{C}(18)-\mathrm{C}(16)-\mathrm{P}(1)$ & $113.6(2)$ \\
\hline $\mathrm{C}(11)-\mathrm{C}(10)-\mathrm{H}(10 \mathrm{~B})$ & 108.2 & $\mathrm{C}(17)-\mathrm{C}(16)-\mathrm{H}(16 \mathrm{~A})$ & 106.8 \\
\hline $\mathrm{B}(1)-\mathrm{C}(10)-\mathrm{H}(10 \mathrm{~B})$ & 108.2 & $\mathrm{C}(18)-\mathrm{C}(16)-\mathrm{H}(16 \mathrm{~A})$ & 106.8 \\
\hline $\mathrm{H}(10 \mathrm{~A})-\mathrm{C}(10)-\mathrm{H}(10 \mathrm{~B})$ & 107.4 & $\mathrm{P}(1)-\mathrm{C}(16)-\mathrm{H}(16 \mathrm{~A})$ & 106.8 \\
\hline $\mathrm{C}(10)-\mathrm{C}(11)-\mathrm{H}(11 \mathrm{~A})$ & 109.5 & $\mathrm{C}(16)-\mathrm{C}(17)-\mathrm{H}(17 \mathrm{~A})$ & 109.5 \\
\hline $\mathrm{C}(10)-\mathrm{C}(11)-\mathrm{H}(11 \mathrm{~B})$ & 109.5 & $\mathrm{C}(16)-\mathrm{C}(17)-\mathrm{H}(17 \mathrm{~B})$ & 109.5 \\
\hline $\mathrm{H}(11 \mathrm{~A})-\mathrm{C}(11)-\mathrm{H}(11 \mathrm{~B})$ & 109.5 & $\mathrm{H}(17 \mathrm{~A})-\mathrm{C}(17)-\mathrm{H}(17 \mathrm{~B})$ & 109.5 \\
\hline $\mathrm{C}(10)-\mathrm{C}(11)-\mathrm{H}(11 \mathrm{C})$ & 109.5 & $\mathrm{C}(16)-\mathrm{C}(17)-\mathrm{H}(17 \mathrm{C})$ & 109.5 \\
\hline $\mathrm{H}(11 \mathrm{~A})-\mathrm{C}(11)-\mathrm{H}(11 \mathrm{C})$ & 109.5 & $\mathrm{H}(17 \mathrm{~A})-\mathrm{C}(17)-\mathrm{H}(17 \mathrm{C})$ & 109.5 \\
\hline $\mathrm{H}(11 \mathrm{~B})-\mathrm{C}(11)-\mathrm{H}(11 \mathrm{C})$ & 109.5 & $\mathrm{H}(17 \mathrm{~B})-\mathrm{C}(17)-\mathrm{H}(17 \mathrm{C})$ & 109.5 \\
\hline$C(13)-C(12)-B(1)$ & $113.8(3)$ & $\mathrm{C}(16)-\mathrm{C}(18)-\mathrm{H}(18 \mathrm{~A})$ & 109.5 \\
\hline $\mathrm{C}(13)-\mathrm{C}(12)-\mathrm{H}(12 \mathrm{~A})$ & 108.8 & $\mathrm{C}(16)-\mathrm{C}(18)-\mathrm{H}(18 \mathrm{~B})$ & 109.5 \\
\hline $\mathrm{B}(1)-\mathrm{C}(12)-\mathrm{H}(12 \mathrm{~A})$ & 108.8 & $\mathrm{H}(18 \mathrm{~A})-\mathrm{C}(18)-\mathrm{H}(18 \mathrm{~B})$ & 109.5 \\
\hline $\mathrm{C}(13)-\mathrm{C}(12)-\mathrm{H}(12 \mathrm{~B})$ & 108.8 & $\mathrm{C}(16)-\mathrm{C}(18)-\mathrm{H}(18 \mathrm{C})$ & 109.5 \\
\hline $\mathrm{B}(1)-\mathrm{C}(12)-\mathrm{H}(12 \mathrm{~B})$ & 108.8 & $\mathrm{H}(18 \mathrm{~A})-\mathrm{C}(18)-\mathrm{H}(18 \mathrm{C})$ & 109.5 \\
\hline $\mathrm{H}(12 \mathrm{~A})-\mathrm{C}(12)-\mathrm{H}(12 \mathrm{~B})$ & 107.7 & $\mathrm{H}(18 \mathrm{~B})-\mathrm{C}(18)-\mathrm{H}(18 \mathrm{C})$ & 109.5 \\
\hline$C(12)-C(13)-H(13 A)$ & 109.5 & $\mathrm{C}(21)-\mathrm{C}(19)-\mathrm{C}(20)$ & $110.8(3)$ \\
\hline $\mathrm{C}(12)-\mathrm{C}(13)-\mathrm{H}(13 \mathrm{~B})$ & 109.5 & $\mathrm{C}(21)-\mathrm{C}(19)-\mathrm{P}(1)$ & $112.8(2)$ \\
\hline $\mathrm{H}(13 \mathrm{~A})-\mathrm{C}(13)-\mathrm{H}(13 \mathrm{~B})$ & 109.5 & $\mathrm{C}(20)-\mathrm{C}(19)-\mathrm{P}(1)$ & $110.1(2)$ \\
\hline $\mathrm{C}(12)-\mathrm{C}(13)-\mathrm{H}(13 \mathrm{C})$ & 109.5 & $\mathrm{C}(21)-\mathrm{C}(19)-\mathrm{H}(19 \mathrm{~A})$ & 107.6 \\
\hline $\mathrm{H}(13 \mathrm{~A})-\mathrm{C}(13)-\mathrm{H}(13 \mathrm{C})$ & 109.5 & $\mathrm{C}(20)-\mathrm{C}(19)-\mathrm{H}(19 \mathrm{~A})$ & 107.6 \\
\hline $\mathrm{H}(13 \mathrm{~B})-\mathrm{C}(13)-\mathrm{H}(13 \mathrm{C})$ & 109.5 & $\mathrm{P}(1)-\mathrm{C}(19)-\mathrm{H}(19 \mathrm{~A})$ & 107.6 \\
\hline$C(15)-C(14)-P(1)$ & $113.3(2)$ & $\mathrm{C}(19)-\mathrm{C}(20)-\mathrm{H}(20 \mathrm{~A})$ & 109.5 \\
\hline $\mathrm{C}(15)-\mathrm{C}(14)-\mathrm{H}(14 \mathrm{~A})$ & 108.9 & $\mathrm{C}(19)-\mathrm{C}(20)-\mathrm{H}(20 \mathrm{~B})$ & 109.5 \\
\hline $\mathrm{P}(1)-\mathrm{C}(14)-\mathrm{H}(14 \mathrm{~A})$ & 108.9 & $\mathrm{H}(20 \mathrm{~A})-\mathrm{C}(20)-\mathrm{H}(20 \mathrm{~B})$ & 109.5 \\
\hline $\mathrm{C}(15)-\mathrm{C}(14)-\mathrm{H}(14 \mathrm{~B})$ & 108.9 & $\mathrm{C}(19)-\mathrm{C}(20)-\mathrm{H}(20 \mathrm{C})$ & 109.5 \\
\hline $\mathrm{P}(1)-\mathrm{C}(14)-\mathrm{H}(14 \mathrm{~B})$ & 108.9 & $\mathrm{H}(20 \mathrm{~A})-\mathrm{C}(20)-\mathrm{H}(20 \mathrm{C})$ & 109.5 \\
\hline $\mathrm{H}(14 \mathrm{~A})-\mathrm{C}(14)-\mathrm{H}(14 \mathrm{~B})$ & 107.7 & H(20B)-C(20)-H(20C) & 109.5 \\
\hline $\mathrm{C}(14)-\mathrm{C}(15)-\mathrm{P}(2)$ & $111.5(2)$ & $\mathrm{C}(19)-\mathrm{C}(21)-\mathrm{H}(21 \mathrm{~A})$ & 109.5 \\
\hline $\mathrm{C}(14)-\mathrm{C}(15)-\mathrm{H}(15 \mathrm{~A})$ & 109.3 & $\mathrm{C}(19)-\mathrm{C}(21)-\mathrm{H}(21 \mathrm{~B})$ & 109.5 \\
\hline $\mathrm{P}(2)-\mathrm{C}(15)-\mathrm{H}(15 \mathrm{~A})$ & 109.3 & $\mathrm{H}(21 \mathrm{~A})-\mathrm{C}(21)-\mathrm{H}(21 \mathrm{~B})$ & 109.5 \\
\hline $\mathrm{C}(14)-\mathrm{C}(15)-\mathrm{H}(15 \mathrm{~B})$ & 109.3 & $\mathrm{C}(19)-\mathrm{C}(21)-\mathrm{H}(21 \mathrm{C})$ & 109.5 \\
\hline $\mathrm{P}(2)-\mathrm{C}(15)-\mathrm{H}(15 \mathrm{~B})$ & 109.3 & $\mathrm{H}(21 \mathrm{~A})-\mathrm{C}(21)-\mathrm{H}(21 \mathrm{C})$ & 109.5 \\
\hline
\end{tabular}




$\begin{array}{llll}\mathrm{H}(21 \mathrm{~B})-\mathrm{C}(21)-\mathrm{H}(21 \mathrm{C}) & 109.5 & \mathrm{C}(27)-\mathrm{C}(25)-\mathrm{C}(26) & 110.6(3) \\ \mathrm{C}(24)-\mathrm{C}(22)-\mathrm{C}(23) & 111.8(3) & \mathrm{C}(27)-\mathrm{C}(25)-\mathrm{P}(2) & 111.7(2) \\ \mathrm{C}(24)-\mathrm{C}(22)-\mathrm{P}(2) & 114.0(2) & \mathrm{C}(26)-\mathrm{C}(25)-\mathrm{P}(2) & 110.9(2) \\ \mathrm{C}(23)-\mathrm{C}(22)-\mathrm{P}(2) & 109.6(2) & \mathrm{C}(27)-\mathrm{C}(25)-\mathrm{H}(25 \mathrm{~A}) & 107.8 \\ \mathrm{C}(24)-\mathrm{C}(22)-\mathrm{H}(22 \mathrm{~A}) & 107.0 & \mathrm{C}(26)-\mathrm{C}(25)-\mathrm{H}(25 \mathrm{~A}) & 107.8 \\ \mathrm{C}(23)-\mathrm{C}(22)-\mathrm{H}(22 \mathrm{~A}) & 107.0 & \mathrm{P}(2)-\mathrm{C}(25)-\mathrm{H}(25 \mathrm{~A}) & 107.8 \\ \mathrm{P}(2)-\mathrm{C}(22)-\mathrm{H}(22 \mathrm{~A}) & 107.0 & \mathrm{C}(25)-\mathrm{C}(26)-\mathrm{H}(26 \mathrm{~A}) & 109.5 \\ \mathrm{C}(22)-\mathrm{C}(23)-\mathrm{H}(23 \mathrm{~A}) & 109.5 & \mathrm{C}(25)-\mathrm{C}(26)-\mathrm{H}(26 \mathrm{~B}) & 109.5 \\ \mathrm{C}(22)-\mathrm{C}(23)-\mathrm{H}(23 \mathrm{~B}) & 109.5 & \mathrm{H}(26 \mathrm{~A})-\mathrm{C}(26)-\mathrm{H}(26 \mathrm{~B}) & 109.5 \\ \mathrm{H}(23 \mathrm{~A})-\mathrm{C}(23)-\mathrm{H}(23 \mathrm{~B}) & 109.5 & \mathrm{C}(25)-\mathrm{C}(26)-\mathrm{H}(26 \mathrm{C}) & 109.5 \\ \mathrm{C}(22)-\mathrm{C}(23)-\mathrm{H}(23 \mathrm{C}) & 109.5 & \mathrm{H}(26 \mathrm{~A})-\mathrm{C}(26)-\mathrm{H}(26 \mathrm{C}) & 109.5 \\ \mathrm{H}(23 \mathrm{~A})-\mathrm{C}(23)-\mathrm{H}(23 \mathrm{C}) & 109.5 & \mathrm{H}(26 \mathrm{~B})-\mathrm{C}(26)-\mathrm{H}(26 \mathrm{C}) & 109.5 \\ \mathrm{H}(23 \mathrm{~B})-\mathrm{C}(23)-\mathrm{H}(23 \mathrm{C}) & 109.5 & \mathrm{C}(25)-\mathrm{C}(27)-\mathrm{H}(27 \mathrm{~A}) & 109.5 \\ \mathrm{C}(22)-\mathrm{C}(24)-\mathrm{H}(24 \mathrm{~A}) & 109.5 & \mathrm{C}(25)-\mathrm{C}(27)-\mathrm{H}(27 \mathrm{~B}) & 109.5 \\ \mathrm{C}(22)-\mathrm{C}(24)-\mathrm{H}(24 \mathrm{~B}) & 109.5 & \mathrm{H}(27 \mathrm{~A})-\mathrm{C}(27)-\mathrm{H}(27 \mathrm{~B}) & 109.5 \\ \mathrm{H}(24 \mathrm{~A})-\mathrm{C}(24)-\mathrm{H}(24 \mathrm{~B}) & 109.5 & \mathrm{C}(25)-\mathrm{C}(27)-\mathrm{H}(27 \mathrm{C}) & 109.5 \\ \mathrm{C}(22)-\mathrm{C}(24)-\mathrm{H}(24 \mathrm{C}) & 109.5 & \mathrm{H}(27 \mathrm{~A})-\mathrm{C}(27)-\mathrm{H}(27 \mathrm{C}) & 109.5 \\ \mathrm{H}(24 \mathrm{~A})-\mathrm{C}(24)-\mathrm{H}(24 \mathrm{C}) & 109.5 & \mathrm{H}(27 \mathrm{~B})-\mathrm{C}(27)-\mathrm{H}(27 \mathrm{C}) & 109.5 \\ \mathrm{H}(24 \mathrm{~B})-\mathrm{C}(24)-\mathrm{H}(24 \mathrm{C}) & 109.5 & & \\ & & & \end{array}$


Table S10. Anisotropic displacement parameters $\left(\AA^{2} \times 10^{3}\right)$ for jonlm36 (3b). The anisotropic displacement factor exponent takes the form: $-2 \pi^{2}\left[\mathrm{~h}^{2} \mathrm{a}^{* 2} \mathrm{U}_{11}+\ldots+2 \mathrm{hk} \mathrm{a} * \mathrm{~b}^{*} \mathrm{U}_{12}\right]$

\begin{tabular}{|c|c|c|c|c|c|c|}
\hline- & $\mathrm{U}_{11}$ & $\mathrm{U}_{22}$ & $\mathrm{U}_{33}$ & $\mathrm{U}_{23}$ & $\mathrm{U}_{13}$ & $\mathrm{U}_{12}$ \\
\hline \multicolumn{7}{|l|}{ - } \\
\hline $\mathrm{Pd} 1$ & $15(1)$ & $16(1)$ & $15(1)$ & $0(1)$ & $2(1)$ & $1(1)$ \\
\hline P1 & $17(1)$ & $17(1)$ & $15(1)$ & $-1(1)$ & $1(1)$ & $1(1)$ \\
\hline $\mathrm{P} 2$ & $15(1)$ & $17(1)$ & $17(1)$ & $1(1)$ & $2(1)$ & $-1(1)$ \\
\hline N1 & $22(2)$ & $17(1)$ & $18(1)$ & $1(1)$ & $1(1)$ & $1(1)$ \\
\hline B1 & $18(2)$ & $18(2)$ & $19(2)$ & $-2(2)$ & $4(2)$ & $3(2)$ \\
\hline $\mathrm{C} 1$ & $20(2)$ & $21(2)$ & $19(2)$ & $2(1)$ & $-2(1)$ & $-2(1)$ \\
\hline $\mathrm{C} 2$ & $20(2)$ & $19(2)$ & $15(2)$ & $-1(1)$ & $4(1)$ & $3(1)$ \\
\hline $\mathrm{C} 3$ & $22(2)$ & $16(2)$ & $19(2)$ & $-1(1)$ & $-1(1)$ & $2(1)$ \\
\hline $\mathrm{C} 4$ & $23(2)$ & $20(2)$ & $17(2)$ & $-1(1)$ & $4(1)$ & $-2(1)$ \\
\hline $\mathrm{C} 5$ & $32(2)$ & $18(2)$ & $20(2)$ & $3(1)$ & $5(2)$ & $3(2)$ \\
\hline C6 & $28(2)$ & $23(2)$ & $22(2)$ & $3(2)$ & $-2(2)$ & $2(2)$ \\
\hline $\mathrm{C} 7$ & $24(2)$ & $22(2)$ & $24(2)$ & $3(2)$ & $1(2)$ & $0(2)$ \\
\hline $\mathrm{C} 8$ & $32(2)$ & $24(2)$ & $19(2)$ & $0(2)$ & $1(2)$ & $4(2)$ \\
\hline C9 & $58(3)$ & $42(2)$ & $28(2)$ & $-1(2)$ & $-16(2)$ & $12(2)$ \\
\hline $\mathrm{C} 10$ & $20(2)$ & $22(2)$ & $21(2)$ & $0(1)$ & $3(1)$ & $2(1)$ \\
\hline $\mathrm{C} 11$ & $32(2)$ & $42(2)$ & $27(2)$ & $5(2)$ & $-9(2)$ & $2(2)$ \\
\hline $\mathrm{C} 12$ & $30(2)$ & $21(2)$ & $24(2)$ & $-1(2)$ & $11(2)$ & $0(2)$ \\
\hline $\mathrm{C} 13$ & $32(2)$ & $24(2)$ & $35(2)$ & $-4(2)$ & $18(2)$ & $-2(2)$ \\
\hline $\mathrm{C} 14$ & $23(2)$ & $17(2)$ & $18(2)$ & $0(1)$ & $3(1)$ & $0(1)$ \\
\hline $\mathrm{C} 15$ & $21(2)$ & $21(2)$ & $17(2)$ & $5(1)$ & $2(1)$ & $0(1)$ \\
\hline $\mathrm{C} 16$ & $20(2)$ & $21(2)$ & $19(2)$ & $-1(1)$ & $2(1)$ & $-1(1)$ \\
\hline $\mathrm{C} 17$ & $23(2)$ & $30(2)$ & $24(2)$ & $-1(2)$ & $3(2)$ & $-5(2)$ \\
\hline $\mathrm{C} 18$ & $22(2)$ & $28(2)$ & $30(2)$ & $-2(2)$ & $6(2)$ & $-1(2)$ \\
\hline C19 & $27(2)$ & $21(2)$ & $16(2)$ & $0(1)$ & $2(1)$ & $0(2)$ \\
\hline $\mathrm{C} 20$ & $29(2)$ & $31(2)$ & $25(2)$ & $-3(2)$ & $-6(2)$ & $2(2)$ \\
\hline $\mathrm{C} 21$ & $34(2)$ & $26(2)$ & $18(2)$ & $0(2)$ & $3(2)$ & $0(2)$ \\
\hline $\mathrm{C} 22$ & $21(2)$ & $25(2)$ & $17(2)$ & $0(1)$ & $4(1)$ & $1(1)$ \\
\hline $\mathrm{C} 23$ & $25(2)$ & $36(2)$ & $22(2)$ & $-1(2)$ & $1(2)$ & $-7(2)$ \\
\hline
\end{tabular}




$\begin{array}{lllllll}\mathrm{C} 24 & 37(2) & 27(2) & 19(2) & 3(2) & 3(2) & -2(2) \\ \mathrm{C} 25 & 17(2) & 24(2) & 22(2) & 0(1) & 3(1) & -2(1) \\ \mathrm{C} 26 & 22(2) & 45(2) & 28(2) & -2(2) & -1(2) & -7(2) \\ \mathrm{C} 27 & 17(2) & 30(2) & 51(3) & -7(2) & 6(2) & -5(2)\end{array}$


Table S11. Hydrogen coordinates $\left(\times 10^{4}\right)$ and isotropic displacement parameters $\left(\AA^{2} \times 10^{3}\right)$ for jonlm36 (3b).

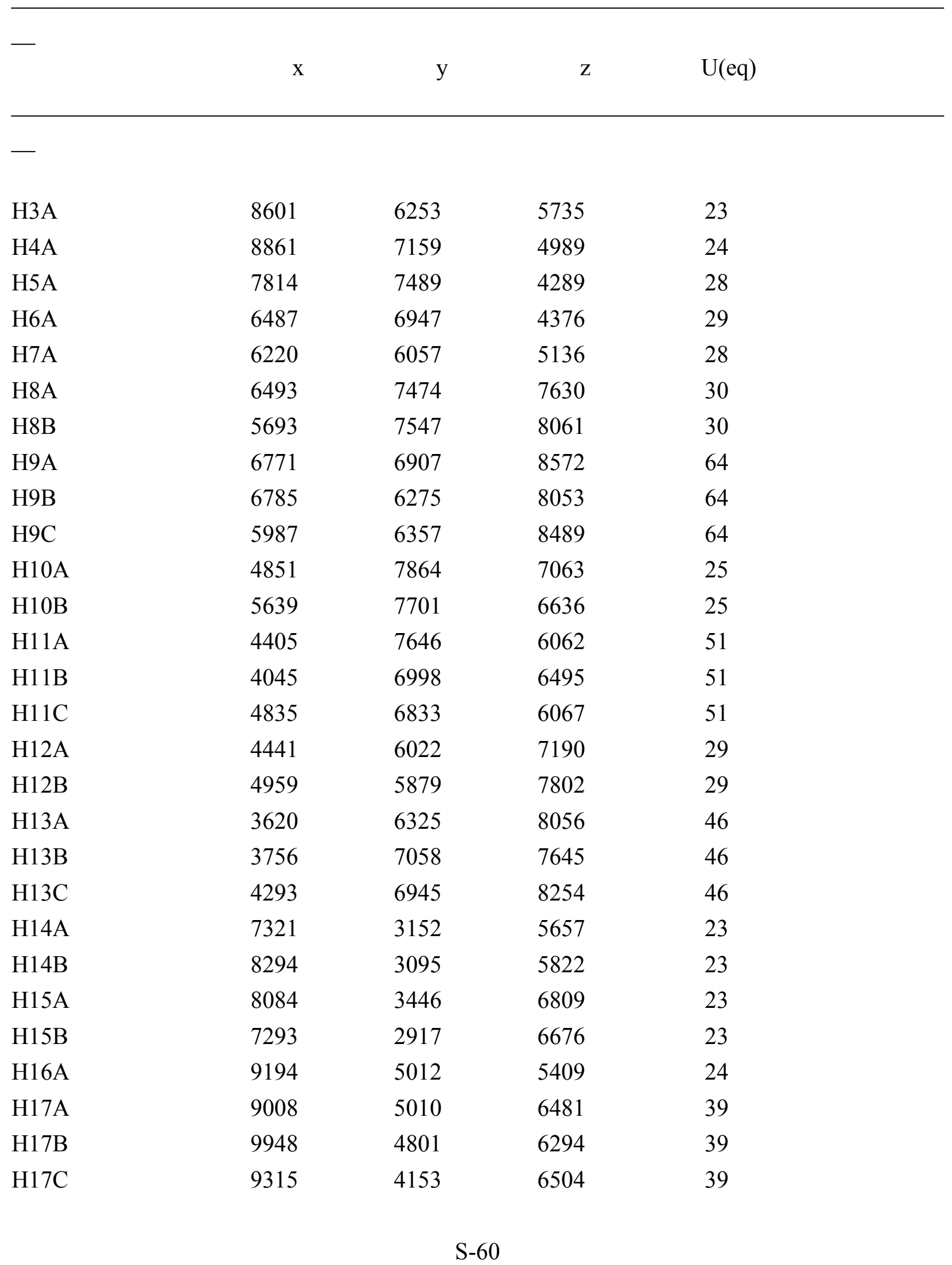




\begin{tabular}{|c|c|c|c|}
\hline $\mathrm{H} 18 \mathrm{~A}$ & 9450 & 3889 & 4877 \\
\hline H18B & 9540 & 3442 & 5506 \\
\hline $\mathrm{H} 18 \mathrm{C}$ & 10211 & 4072 & 5327 \\
\hline H19A & 8006 & 3736 & 4620 \\
\hline $\mathrm{H} 20 \mathrm{~A}$ & 6570 & 3751 & 4864 \\
\hline H20B & 6704 & 4084 & 4194 \\
\hline $\mathrm{H} 20 \mathrm{C}$ & 6533 & 4640 & 4753 \\
\hline $\mathrm{H} 21 \mathrm{~A}$ & 8751 & 4842 & 4412 \\
\hline $\mathrm{H} 21 \mathrm{~B}$ & 7918 & 5334 & 4482 \\
\hline $\mathrm{H} 21 \mathrm{C}$ & 8009 & 4757 & 3927 \\
\hline $\mathrm{H} 22 \mathrm{~A}$ & 6591 & 4749 & 7708 \\
\hline $\mathrm{H} 23 \mathrm{~A}$ & 7891 & 5234 & 7409 \\
\hline H23B & 8329 & 4438 & 7526 \\
\hline $\mathrm{H} 23 \mathrm{C}$ & 7959 & 4908 & 8087 \\
\hline $\mathrm{H} 24 \mathrm{~A}$ & 6398 & 3469 & 7937 \\
\hline $\mathrm{H} 24 \mathrm{~B}$ & 7021 & 3850 & 8417 \\
\hline $\mathrm{H} 24 \mathrm{C}$ & 7385 & 3329 & 7886 \\
\hline $\mathrm{H} 25 \mathrm{~A}$ & 5766 & 3332 & 6990 \\
\hline $\mathrm{H} 26 \mathrm{~A}$ & 5991 & 3137 & 5947 \\
\hline H26B & 5625 & 3953 & 5792 \\
\hline $\mathrm{H} 26 \mathrm{C}$ & 5021 & 3314 & 6058 \\
\hline $\mathrm{H} 27 \mathrm{~A}$ & 5259 & 4482 & 7360 \\
\hline H27B & 4579 & 4099 & 6924 \\
\hline $\mathrm{H} 27 \mathrm{C}$ & 5138 & 4782 & 6679 \\
\hline
\end{tabular}


Table S12. Torsion angles $\left[^{\circ}\right]$ for jonlm36 (3b).

\begin{tabular}{|c|c|c|c|}
\hline $\mathrm{C} 7-\mathrm{C} 2-\mathrm{C} 3-\mathrm{C} 4$ & $-2.6(5)$ & C16-P1-C19-C20 & $-173.0(2)$ \\
\hline $\mathrm{Pd1-C2-C3-C4}$ & $175.5(2)$ & C14-P1-C19-C20 & $76.1(3)$ \\
\hline $\mathrm{C} 2-\mathrm{C} 3-\mathrm{C} 4-\mathrm{C} 5$ & $0.1(5)$ & Pd1-P1-C19-C20 & $-43.6(3)$ \\
\hline C3-C4-C5-C6 & $1.9(5)$ & C15-P2-C22-C24 & $-53.3(3)$ \\
\hline C4-C5-C6-C7 & $-1.2(5)$ & $\mathrm{C} 25-\mathrm{P} 2-\mathrm{C} 22-\mathrm{C} 24$ & $58.2(3)$ \\
\hline C3-C2-C7-C6 & $3.3(5)$ & $\mathrm{Pd} 1-\mathrm{P} 2-\mathrm{C} 22-\mathrm{C} 24$ & $-172.2(2)$ \\
\hline Pd1-C2-C7-C6 & $-174.9(3)$ & C15-P2-C22-C23 & $73.0(3)$ \\
\hline C5-C6-C7-C2 & $-1.4(6)$ & $\mathrm{C} 25-\mathrm{P} 2-\mathrm{C} 22-\mathrm{C} 23$ & $-175.5(2)$ \\
\hline N1-B1-C8-C9 & $-62.0(4)$ & Pd1-P2-C22-C23 & $-46.0(3)$ \\
\hline C10-B1-C8-C9 & $-175.8(3)$ & C15-P2-C25-C27 & $173.2(3)$ \\
\hline C12-B1-C8-C9 & $53.8(4)$ & $\mathrm{C} 22-\mathrm{P} 2-\mathrm{C} 25-\mathrm{C} 27$ & $61.3(3)$ \\
\hline N1-B1-C10-C11 & $68.4(4)$ & $\mathrm{Pd} 1-\mathrm{P} 2-\mathrm{C} 25-\mathrm{C} 27$ & $-67.3(3)$ \\
\hline C12-B1-C10-C11 & $-46.8(4)$ & $\mathrm{C} 15-\mathrm{P} 2-\mathrm{C} 25-\mathrm{C} 26$ & $-63.0(3)$ \\
\hline C8-B1-C10-C11 & $-177.3(3)$ & C22-P2-C25-C26 & $-174.8(3)$ \\
\hline N1-B1-C12-C13 & $-177.1(3)$ & Pd1-P2-C25-C26 & $56.5(3)$ \\
\hline C10-B1-C12-C13 & $-61.9(4)$ & & \\
\hline C8-B1-C12-C13 & $66.6(4)$ & & \\
\hline C16-P1-C14-C15 & $93.3(3)$ & & \\
\hline C19-P1-C14-C15 & $-156.8(2)$ & & \\
\hline Pd1-P1-C14-C15 & $-29.9(3)$ & & \\
\hline P1-C14-C15-P2 & $39.7(3)$ & & \\
\hline C25-P2-C15-C14 & $93.8(3)$ & & \\
\hline C22-P2-C15-C14 & $-154.5(2)$ & & \\
\hline Pd1-P2-C15-C14 & $-31.2(3)$ & & \\
\hline C14-P1-C16-C17 & $-70.3(3)$ & & \\
\hline C19-P1-C16-C17 & $-179.3(2)$ & & \\
\hline Pd1-P1-C16-C17 & $48.8(3)$ & & \\
\hline C14-P1-C16-C18 & $56.0(3)$ & & \\
\hline C19-P1-C16-C18 & $-53.0(3)$ & & \\
\hline Pd1-P1-C16-C18 & $175.1(2)$ & & \\
\hline C16-P1-C19-C21 & $-48.6(3)$ & & \\
\hline C14-P1-C19-C21 & $-159.5(3)$ & & \\
\hline Pd1-P1-C19-C21 & $80.7(3)$ & & \\
\hline
\end{tabular}


REFERENCE NUMBER: jonlm37 (2a')

CRYSTAL STRUCTURE REPORT

$\mathrm{C}_{34} \mathrm{H}_{50} \mathrm{~B} \mathrm{~N} \mathrm{P} 2 \mathrm{Pd}$

Report prepared for:

L. Munjanja, Prof. W. Jones

March 08, 2016

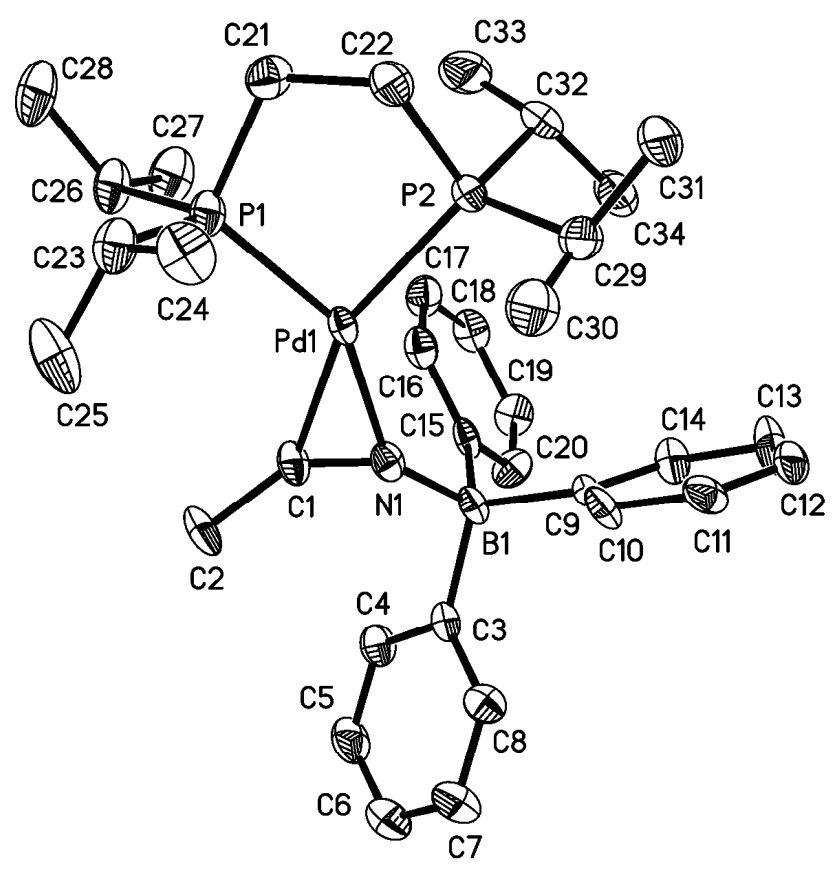

William W. Brennessel

X-ray Crystallographic Facility

Department of Chemistry, University of Rochester

120 Trustee Road

Rochester, NY 14627 


\section{Data collection}

A crystal $\left(0.45 \times 0.20 \times 0.12 \mathrm{~mm}^{3}\right)$ was placed onto the tip of a thin glass optical fiber and mounted on a Bruker SMART APEX II CCD platform diffractometer for a data collection at 100.0(5) K. ${ }^{1}$ A preliminary set of cell constants and an orientation matrix were calculated from reflections harvested from three orthogonal wedges of reciprocal space. The full data collection was carried out using MoKa radiation (graphite monochromator) with a frame time of 45 seconds and a detector distance of $4.01 \mathrm{~cm}$. A randomly oriented region of reciprocal space was surveyed: five major sections of frames were collected with $0.50^{\circ}$ steps in $\omega$ at five different $\phi$ settings and a detector position of $-38^{\circ}$ in $2 \theta$. The intensity data were corrected for absorption. ${ }^{2}$ Final cell constants were calculated from the xyz centroids of 9397 strong reflections from the actual data collection after integration. ${ }^{3}$ See Table S13 for additional crystal and refinement information.

\section{Structure solution and refinement}

The structure was solved using SHELXT-2014/5 and refined using SHELXL-2014/7. 5 The space group $P 2_{1} / c$ was determined based on systematic absences. A direct-methods solution was calculated which provided most nonhydrogen atoms from the E-map. Full-matrix least squares / difference Fourier cycles were performed which located the remaining non-hydrogen atoms. All non-hydrogen atoms were refined with anisotropic displacement parameters. All hydrogen atoms were placed in ideal positions and refined as riding atoms with relative isotropic displacement parameters.

The refinement stalled at $R 1=0.244$, at which point twin modeling was required. After the non-merohedral twin

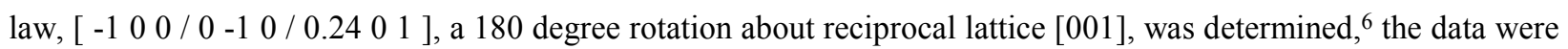
re-integrated, ${ }^{3}$ and a new absorption correction was applied. ${ }^{7}$ There were 5375 unique reflections solely in the first component, 5012 unique reflections solely in the second component, and 18836 unique overlapping reflections. The mass ratio of the two components refined to 0.50:0.50.

The final full matrix least squares refinement converged to $R 1=0.0663\left(F^{2}, I>2 \sigma(I)\right)$ and $w R 2=0.1352\left(F^{2}\right.$, all data).

\section{Structure description}

The structure is the one suggested. The asymmetric unit contains two independent palladium complexes in general positions. In one molecule the diphosphane ligand and the methyl end of the coordinated alkyne ligand are modeled as disordered over two positions (0.61:0.39). The disphosphane ligand appears to be disordered over additional positions, but no attempted model incorporating three or more positions improved the structure significantly. 
Unless noted otherwise all structural diagrams containing thermal displacement ellipsoids are drawn at the $50 \%$ probability level.

Data collection, structure solution, and structure refinement were conducted at the X-ray Crystallographic Facility, B51 Hutchison Hall, Department of Chemistry, University of Rochester. All publications arising from this report MUST either 1) include William W. Brennessel as a coauthor or 2) acknowledge William W. Brennessel and the Xray Crystallographic Facility of the Department of Chemistry at the University of Rochester.

1 APEX3, version 2015.9-0; Bruker AXS: Madison, WI, 2015.

2 Sheldrick, G. M. SADABS, version 2014/5; J. Appl. Cryst. 2015, 48, 3-10.

3 SAINT, version 8.34A; Bruker AXS: Madison, WI, 2013.

4 Sheldrick, G. M. SHELXT-2014/5; University of Göttingen: Göttingen, Germany, 2014.

5 Sheldrick, G. M. SHELXL-2014/7; Acta. Cryst. 2015, C71, 3-8.

6 a) Parsons, S.; Gould, B.; Cooper, R.; Farrugia, L. ROTAX; University of Edinburgh: Edinburgh, Scotland, 2003;

b) Sheldrick, G. M. CELL_NOW: A program that analyzes a list of reflections to find a cell and orientation matrix despite the presence of several twin domains or other junk, version 2008/2; University of Göttingen: Göttingen, Germany, 2008.

7 Sheldrick, G. M. TWINABS, version 2012/1; University of Göttingen: Göttingen, Germany, 2012.

Some equations of interest:

$$
\begin{gathered}
R_{\mathrm{int}}=\Sigma\left|F_{\mathrm{o}}^{2}-<F_{\mathrm{o}}{ }^{2}>\right| / \Sigma\left|F_{\mathrm{o}}{ }^{2}\right| \\
R 1=\Sigma|| F_{\mathrm{o}}|-| F_{\mathrm{c}} \| / \Sigma\left|F_{\mathrm{o}}\right| \\
w R 2=\left[\Sigma\left[w\left(F_{\mathrm{o}}{ }^{2}-F_{\mathrm{c}}{ }^{2}\right)^{2}\right] / \Sigma\left[w\left(F_{\mathrm{o}}{ }^{2}\right)^{2}\right]\right]^{1 / 2} \\
\text { where } w=1 /\left[\sigma^{2}\left(F_{\mathrm{o}}{ }^{2}\right)+(a P)^{2}+b P\right] \text { and } \\
P=1 / 3 \max \left(0, F_{\mathrm{o}}{ }^{2}\right)+2 / 3 F_{\mathrm{c}}{ }^{2} \\
\mathrm{GOF}=S=\left[\Sigma\left[w\left(F_{\mathrm{o}}{ }^{2}-F_{\mathrm{c}}{ }^{2}\right)^{2}\right] /(m-n)\right]^{1 / 2}
\end{gathered}
$$

where $m=$ number of reflections and $n=$ number of parameters 

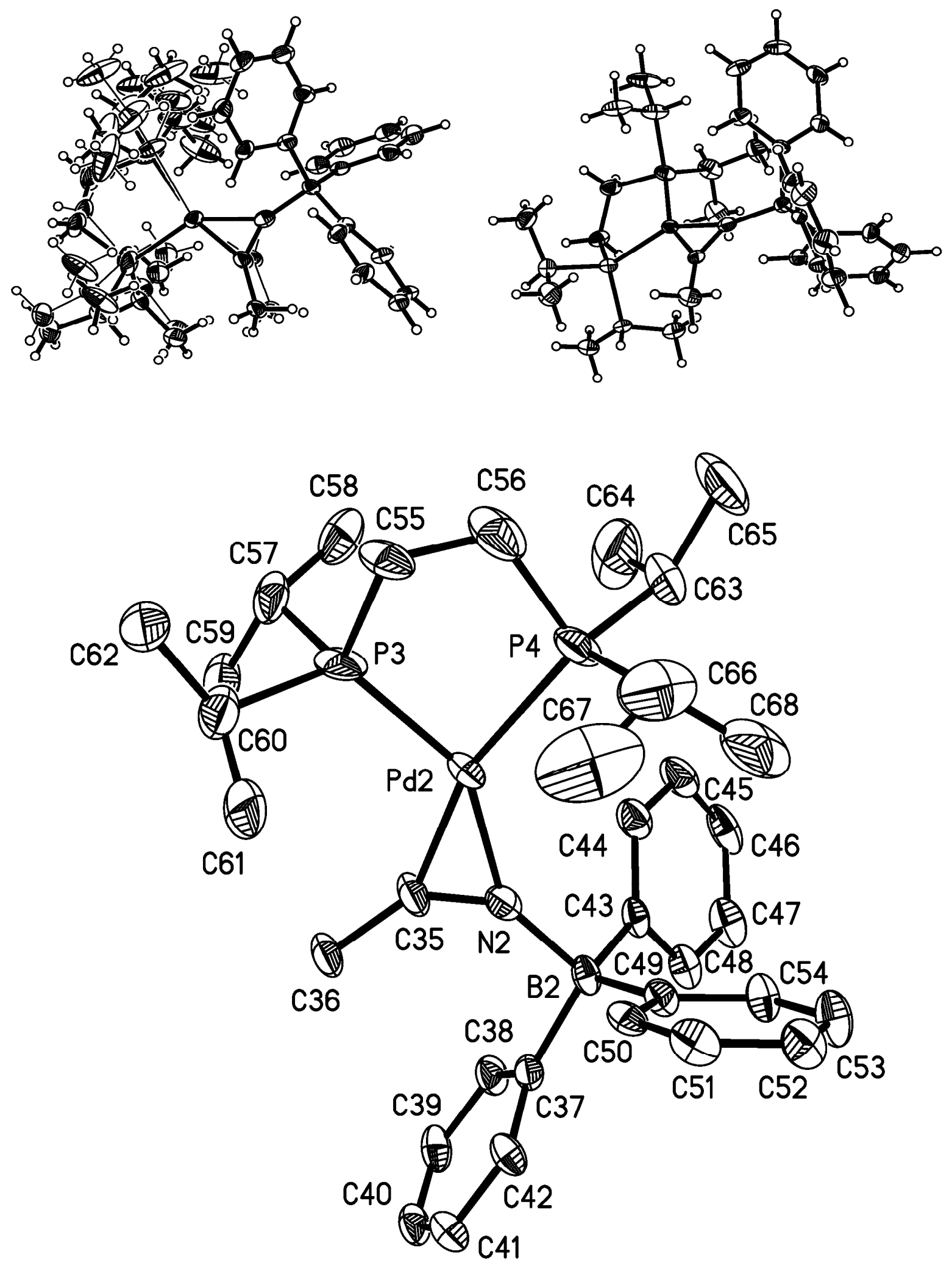
Table S13. Crystal data and structure refinement for jonlm37 (2a')

\begin{tabular}{|c|c|c|}
\hline Identification code & \multicolumn{2}{|l|}{ jonlm 37} \\
\hline Empirical formula & \multicolumn{2}{|c|}{ C34 H50 B N P2 Pd } \\
\hline Formula weight & \multicolumn{2}{|l|}{651.90} \\
\hline Temperature & \multicolumn{2}{|l|}{$100.0(5) \mathrm{K}$} \\
\hline Wavelength & \multicolumn{2}{|l|}{$0.71073 \AA$} \\
\hline Crystal system & \multicolumn{2}{|l|}{ monoclinic } \\
\hline Space group & \multicolumn{2}{|l|}{$P 2_{1} / c$} \\
\hline \multirow[t]{3}{*}{ Unit cell dimensions } & $a=22.130(3) \AA$ & $\alpha=90^{\circ}$ \\
\hline & $b=18.638(2) \AA$ & $\beta=94.965(3)^{\circ}$ \\
\hline & $c=16.427(2) \AA$ & $\gamma=90^{\circ}$ \\
\hline Volume & \multicolumn{2}{|l|}{$6750.3(15) \AA^{3}$} \\
\hline$Z$ & \multicolumn{2}{|l|}{8} \\
\hline Density (calculated) & \multicolumn{2}{|l|}{$1.283 \mathrm{Mg} / \mathrm{m}^{3}$} \\
\hline Absorption coefficient & \multicolumn{2}{|l|}{$0.667 \mathrm{~mm}^{-1}$} \\
\hline$F(000)$ & \multicolumn{2}{|l|}{2736} \\
\hline Crystal color, morphology & \multicolumn{2}{|l|}{ pale yellow, block } \\
\hline Crystal size & \multicolumn{2}{|c|}{$0.45 \times 0.20 \times 0.12 \mathrm{~mm}^{3}$} \\
\hline Theta range for data collection & \multicolumn{2}{|l|}{1.431 to $29.727^{\circ}$} \\
\hline Index ranges & \multicolumn{2}{|c|}{$-30 \leq h \leq 30,0 \leq k \leq 25,0 \leq l \leq 22$} \\
\hline Reflections collected & \multicolumn{2}{|l|}{123451} \\
\hline Independent reflections & \multicolumn{2}{|c|}{$19464[R($ int $)=0.1099]$} \\
\hline Observed reflections & \multicolumn{2}{|l|}{15488} \\
\hline Completeness to theta $=29.575^{\circ}$ & \multicolumn{2}{|l|}{$99.9 \%$} \\
\hline Absorption correction & \multicolumn{2}{|l|}{ Multi-scan } \\
\hline Max. and min. transmission & \multicolumn{2}{|l|}{0.7459 and 0.5527} \\
\hline Refinement method & \multicolumn{2}{|c|}{ Full-matrix least-squares on $F^{2}$} \\
\hline Data / restraints / parameters & \multicolumn{2}{|l|}{19464 / $210 / 771$} \\
\hline Goodness-of-fit on $F^{2}$ & \multicolumn{2}{|l|}{1.073} \\
\hline Final $R$ indices $[I>2 \operatorname{sigma}(I)]$ & \multicolumn{2}{|c|}{$R 1=0.0663, w R 2=0.1250$} \\
\hline$R$ indices (all data) & \multicolumn{2}{|c|}{$R 1=0.0916, w R 2=0.1352$} \\
\hline Largest diff. peak and hole & \multicolumn{2}{|c|}{1.391 and -1.766 e. $\AA^{-3}$} \\
\hline
\end{tabular}


Table S14. Atomic coordinates $\left(\mathrm{x} 10^{4}\right)$ and equivalent isotropic displacement parameters $\left(\AA^{2} \times 10^{3}\right)$ for jonlm37. (2a') $U_{e q}$ is defined as one third of the trace of the orthogonalized $U_{i j}$ tensor.

\begin{tabular}{|c|c|c|c|c|}
\hline & $\mathrm{x}$ & $\mathrm{y}$ & $\mathrm{z}$ & $\mathrm{U}_{\mathrm{eq}}$ \\
\hline $\mathrm{Pd} 1$ & $422(1)$ & $3030(1)$ & $1085(1)$ & $19(1)$ \\
\hline $\mathrm{P} 1$ & $1374(1)$ & $3434(1)$ & $970(1)$ & $25(1)$ \\
\hline $\mathrm{P} 2$ & $677(1)$ & 1998(1) & $378(1)$ & $26(1)$ \\
\hline N1 & $-462(2)$ & $3226(2)$ & $1410(3)$ & $22(1)$ \\
\hline B1 & $-1149(3)$ & $3029(3)$ & $1178(4)$ & $21(1)$ \\
\hline $\mathrm{C} 1$ & $-106(2)$ & $3688(2)$ & $1689(3)$ & $23(1)$ \\
\hline $\mathrm{C} 2$ & $-134(3)$ & $4361(3)$ & $2168(4)$ & $35(1)$ \\
\hline $\mathrm{C} 3$ & $-1542(2)$ & $3400(3)$ & $1863(3)$ & $20(1)$ \\
\hline $\mathrm{C} 4$ & $-1736(3)$ & $4121(3)$ & $1788(4)$ & $30(1)$ \\
\hline $\mathrm{C} 5$ & $-2047(3)$ & $4461(3)$ & $2382(4)$ & $36(1)$ \\
\hline C6 & $-2171(3)$ & $4086(3)$ & $3076(4)$ & $36(1)$ \\
\hline $\mathrm{C} 7$ & $-1987(3)$ & $3381(3)$ & $3166(4)$ & $36(1)$ \\
\hline $\mathrm{C} 8$ & $-1682(2)$ & $3049(3)$ & $2568(4)$ & $26(1)$ \\
\hline $\mathrm{C} 9$ & $-1191(2)$ & $2151(2)$ & $1179(3)$ & $18(1)$ \\
\hline $\mathrm{C} 10$ & $-861(3)$ & 1734(3) & $1765(3)$ & $27(1)$ \\
\hline $\mathrm{C} 11$ & $-888(3)$ & 991(3) & $1773(4)$ & $29(1)$ \\
\hline $\mathrm{C} 12$ & $-1252(3)$ & $630(3)$ & $1184(4)$ & $32(1)$ \\
\hline $\mathrm{C} 13$ & $-1581(3)$ & $1020(3)$ & $590(5)$ & $41(2)$ \\
\hline $\mathrm{C} 14$ & $-1560(3)$ & $1766(3)$ & $596(4)$ & $29(1)$ \\
\hline $\mathrm{C} 15$ & $-1314(2)$ & $3348(2)$ & 261(3) & $21(1)$ \\
\hline $\mathrm{C} 16$ & $-881(2)$ & $3546(3)$ & $-273(4)$ & $26(1)$ \\
\hline $\mathrm{C} 17$ & $-1039(3)$ & $3770(3)$ & $-1060(4)$ & $31(1)$ \\
\hline $\mathrm{C} 18$ & $-1646(3)$ & $3806(3)$ & $-1369(4)$ & $30(1)$ \\
\hline $\mathrm{C} 19$ & $-2087(3)$ & $3623(3)$ & $-859(4)$ & $31(1)$ \\
\hline $\mathrm{C} 20$ & $-1922(2)$ & $3408(3)$ & $-66(4)$ & $28(1)$ \\
\hline $\mathrm{C} 21$ & $1740(3)$ & $2824(3)$ & $276(4)$ & $30(1)$ \\
\hline $\mathrm{C} 22$ & 1504(3) & $2045(3)$ & $308(4)$ & $31(1)$ \\
\hline $\mathrm{C} 23$ & 1914(3) & $3509(3)$ & 1893(4) & $34(1)$ \\
\hline $\mathrm{C} 24$ & 2020(3) & 2781(3) & $2296(4)$ & $41(2)$ \\
\hline $\mathrm{C} 25$ & $1667(4)$ & 4037(4) & $2486(5)$ & $55(2)$ \\
\hline $\mathrm{C} 26$ & $1406(3)$ & 4321(3) & $464(4)$ & $31(1)$ \\
\hline
\end{tabular}




\begin{tabular}{|c|c|c|c|c|}
\hline $\mathrm{C} 27$ & $918(3)$ & $4384(3)$ & $-248(5)$ & $43(2)$ \\
\hline $\mathrm{C} 28$ & $2022(3)$ & $4519(3)$ & $208(5)$ & $44(2)$ \\
\hline $\mathrm{C} 29$ & $572(3)$ & $1148(3)$ & $941(4)$ & $37(2)$ \\
\hline $\mathrm{C} 30$ & $801(4)$ & $1218(3)$ & $1825(5)$ & $46(2)$ \\
\hline $\mathrm{C} 31$ & $832(4)$ & $485(3)$ & $543(5)$ & $54(2)$ \\
\hline $\mathrm{C} 32$ & $368(3)$ & 1807(3) & $-681(4)$ & $36(1)$ \\
\hline $\mathrm{C} 33$ & 493(3) & $2428(4)$ & $-1240(4)$ & $48(2)$ \\
\hline $\mathrm{C} 34$ & $-318(3)$ & $1655(4)$ & $-711(4)$ & $42(2)$ \\
\hline $\mathrm{Pd} 2$ & $5405(1)$ & $2678(1)$ & $5800(1)$ & $26(1)$ \\
\hline $\mathrm{C} 35$ & $4756(8)$ & $3392(10)$ & $5590(4)$ & $38(2)$ \\
\hline $\mathrm{C} 36$ & $4580(5)$ & $4164(5)$ & $5555(8)$ & $38(2)$ \\
\hline P3 & $6317(3)$ & $3210(5)$ & $6044(7)$ & $38(1)$ \\
\hline C55 & $6873(6)$ & $2590(6)$ & $6560(10)$ & $38(2)$ \\
\hline C56 & $6750(5)$ & $1841(7)$ & $6378(11)$ & $56(3)$ \\
\hline P4 & $5928(2)$ & $1605(2)$ & $6096(2)$ & $39(1)$ \\
\hline C57 & $6671(7)$ & $3617(5)$ & 5192(8) & $42(2)$ \\
\hline C58 & $6749(6)$ & $3054(6)$ & $4548(8)$ & $46(3)$ \\
\hline C59 & $6256(7)$ & $4232(7)$ & $4908(11)$ & $53(4)$ \\
\hline C60 & $6428(4)$ & $4042(5)$ & $6638(7)$ & $44(3)$ \\
\hline C61 & $6007(6)$ & $4009(7)$ & $7310(9)$ & $64(4)$ \\
\hline C62 & $7079(6)$ & $4239(9)$ & $6926(12)$ & $47(4)$ \\
\hline C63 & $5950(7)$ & $890(5)$ & $5339(7)$ & $63(4)$ \\
\hline C64 & $6028(8)$ & $1179(8)$ & 4494(8) & $84(5)$ \\
\hline C65 & $6397(7)$ & $289(6)$ & $5562(12)$ & $98(6)$ \\
\hline C66 & $5746(7)$ & $1159(8)$ & $7042(7)$ & $81(4)$ \\
\hline C67 & $5662(14)$ & $1709(14)$ & $7708(15)$ & $106(7)$ \\
\hline C68 & $5195(6)$ & $674(7)$ & $6871(11)$ & $88(5)$ \\
\hline $\mathrm{C} 35^{\prime}$ & $4720(14)$ & $3345(17)$ & $5377(9)$ & $38(2)$ \\
\hline $\mathrm{C} 36^{\prime}$ & $4589(9)$ & $4114(9)$ & $5094(12)$ & $38(2)$ \\
\hline P3' & $6351(4)$ & $3140(8)$ & $6072(12)$ & $38(1)$ \\
\hline $\mathrm{C} 55^{\prime}$ & $6821(10)$ & $2374(10)$ & $6450(18)$ & $38(2)$ \\
\hline C56' & $6605(7)$ & $1679(10)$ & $6193(16)$ & $56(3)$ \\
\hline P4' & $5753(3)$ & $1590(3)$ & $6336(4)$ & $39(1)$ \\
\hline C57' & $6652(12)$ & $3399(9)$ & $5106(12)$ & $42(2)$ \\
\hline C58' & $6625(10)$ & $2775(10)$ & $4510(14)$ & $46(3)$ \\
\hline C59' & $6394(13)$ & $4046(11)$ & $4634(18)$ & $53(4)$ \\
\hline
\end{tabular}




\begin{tabular}{|c|c|c|c|c|}
\hline $\mathrm{C} 60^{\prime}$ & $6392(7)$ & $3764(9)$ & $6947(11)$ & $44(3)$ \\
\hline C61' & $6077(11)$ & $3574(11)$ & 7701(13) & $64(4)$ \\
\hline C62' & 7031(9) & $4038(16)$ & $7180(20)$ & $47(4)$ \\
\hline C63' & $5726(9)$ & 733(7) & $5776(12)$ & $63(4)$ \\
\hline C64' & $5886(15)$ & $796(14)$ & $4897(14)$ & $84(5)$ \\
\hline C65' & $5084(9)$ & $440(10)$ & $5710(20)$ & $98(6)$ \\
\hline C66' & $5464(12)$ & $1370(12)$ & $7324(11)$ & $81(4)$ \\
\hline C67' & $5580(20)$ & $2056(19)$ & $7810(30)$ & $106(7)$ \\
\hline C68' & $5742(12)$ & $708(12)$ & $7739(18)$ & $88(5)$ \\
\hline $\mathrm{N} 2$ & $4475(2)$ & $2806(2)$ & $5469(3)$ & $26(1)$ \\
\hline B2 & $3819(3)$ & $2432(3)$ & $5332(4)$ & $22(1)$ \\
\hline $\mathrm{C} 37$ & $3339(2)$ & $3107(2)$ & $5295(4)$ & $22(1)$ \\
\hline $\mathrm{C} 38$ & $3169(3)$ & $3475(3)$ & $4568(4)$ & $28(1)$ \\
\hline C39 & 2823(3) & 4104(3) & $4557(4)$ & $33(1)$ \\
\hline $\mathrm{C} 40$ & $2631(2)$ & $4374(3)$ & $5260(4)$ & $31(1)$ \\
\hline C41 & 2774(3) & 4021(3) & $5986(4)$ & $31(1)$ \\
\hline $\mathrm{C} 42$ & $3121(3)$ & $3388(3)$ & $5997(4)$ & $26(1)$ \\
\hline $\mathrm{C} 43$ & $3802(2)$ & 1990(3) & $4475(3)$ & $23(1)$ \\
\hline $\mathrm{C} 44$ & $4317(3)$ & $1757(3)$ & $4128(4)$ & $31(1)$ \\
\hline $\mathrm{C} 45$ & $4286(3)$ & 1343(3) & $3420(4)$ & $37(1)$ \\
\hline $\mathrm{C} 46$ & $3736(3)$ & $1155(3)$ & $3033(4)$ & $38(2)$ \\
\hline $\mathrm{C} 47$ & $3209(3)$ & 1377(3) & $3350(4)$ & $37(2)$ \\
\hline $\mathrm{C} 48$ & $3249(3)$ & $1786(3)$ & $4061(4)$ & $31(1)$ \\
\hline C49 & $3765(3)$ & 1911(3) & $6110(3)$ & $25(1)$ \\
\hline C50 & $3973(2)$ & $2130(3)$ & $6907(4)$ & $26(1)$ \\
\hline C51 & $3925(3)$ & 1703(3) & $7583(4)$ & $32(1)$ \\
\hline C52 & $3667(3)$ & $1020(3)$ & 7493(4) & $33(1)$ \\
\hline C53 & $3478(3)$ & 781(3) & $6711(4)$ & $36(1)$ \\
\hline C54 & $3522(3)$ & $1223(3)$ & $6040(4)$ & $31(1)$ \\
\hline
\end{tabular}


Table S15. Bond lengths $[\AA]$ and angles $\left[{ }^{\circ}\right]$ for jonlm37 (2a').

\begin{tabular}{|c|c|c|c|}
\hline $\operatorname{Pd}(1)-C(1)$ & $2.014(5)$ & $\mathrm{C}(11)-\mathrm{C}(12)$ & $1.379(8)$ \\
\hline $\operatorname{Pd}(1)-\mathrm{N}(1)$ & $2.103(5)$ & $\mathrm{C}(11)-\mathrm{H}(11)$ & 0.9500 \\
\hline $\operatorname{Pd}(1)-\mathrm{P}(1)$ & $2.2602(15)$ & $\mathrm{C}(12)-\mathrm{C}(13)$ & $1.373(9)$ \\
\hline $\mathrm{Pd}(1)-\mathrm{P}(2)$ & $2.3402(14)$ & $\mathrm{C}(12)-\mathrm{H}(12)$ & 0.9500 \\
\hline $\mathrm{P}(1)-\mathrm{C}(21)$ & $1.845(6)$ & $\mathrm{C}(13)-\mathrm{C}(14)$ & $1.391(8)$ \\
\hline $\mathrm{P}(1)-\mathrm{C}(23)$ & $1.854(6)$ & $\mathrm{C}(13)-\mathrm{H}(13)$ & 0.9500 \\
\hline $\mathrm{P}(1)-\mathrm{C}(26)$ & $1.855(5)$ & $\mathrm{C}(14)-\mathrm{H}(14)$ & 0.9500 \\
\hline $\mathrm{P}(2)-\mathrm{C}(22)$ & $1.845(6)$ & $C(15)-C(16)$ & $1.403(8)$ \\
\hline $\mathrm{P}(2)-\mathrm{C}(32)$ & $1.849(6)$ & $C(15)-C(20)$ & $1.410(7)$ \\
\hline $\mathrm{P}(2)-\mathrm{C}(29)$ & $1.859(6)$ & $C(16)-C(17)$ & $1.374(8)$ \\
\hline $\mathrm{N}(1)-\mathrm{C}(1)$ & $1.229(6)$ & $\mathrm{C}(16)-\mathrm{H}(16)$ & 0.9500 \\
\hline $\mathrm{N}(1)-\mathrm{B}(1)$ & $1.579(7)$ & $\mathrm{C}(17)-\mathrm{C}(18)$ & $1.396(8)$ \\
\hline $\mathrm{B}(1)-\mathrm{C}(15)$ & $1.630(8)$ & $\mathrm{C}(17)-\mathrm{H}(17)$ & 0.9500 \\
\hline $\mathrm{B}(1)-\mathrm{C}(3)$ & $1.634(8)$ & $C(18)-C(19)$ & $1.383(8)$ \\
\hline $\mathrm{B}(1)-\mathrm{C}(9)$ & $1.640(7)$ & $\mathrm{C}(18)-\mathrm{H}(18)$ & 0.9500 \\
\hline $\mathrm{C}(1)-\mathrm{C}(2)$ & $1.486(7)$ & $C(19)-C(20)$ & $1.383(8)$ \\
\hline $\mathrm{C}(2)-\mathrm{H}(2 \mathrm{~A})$ & 0.9800 & C(19)-H(19) & 0.9500 \\
\hline $\mathrm{C}(2)-\mathrm{H}(2 \mathrm{~B})$ & 0.9800 & $\mathrm{C}(20)-\mathrm{H}(20)$ & 0.9500 \\
\hline $\mathrm{C}(2)-\mathrm{H}(2 \mathrm{C})$ & 0.9800 & $\mathrm{C}(21)-\mathrm{C}(22)$ & $1.546(7)$ \\
\hline $\mathrm{C}(3)-\mathrm{C}(8)$ & $1.388(8)$ & $\mathrm{C}(21)-\mathrm{H}(21 \mathrm{~A})$ & 0.9900 \\
\hline$C(3)-C(4)$ & $1.413(7)$ & $\mathrm{C}(21)-\mathrm{H}(21 \mathrm{~B})$ & 0.9900 \\
\hline$C(4)-C(5)$ & $1.393(8)$ & $\mathrm{C}(22)-\mathrm{H}(22 \mathrm{~A})$ & 0.9900 \\
\hline $\mathrm{C}(4)-\mathrm{H}(4)$ & 0.9500 & $\mathrm{C}(22)-\mathrm{H}(22 \mathrm{~B})$ & 0.9900 \\
\hline$C(5)-C(6)$ & $1.385(9)$ & $C(23)-C(24)$ & $1.518(8)$ \\
\hline $\mathrm{C}(5)-\mathrm{H}(5)$ & 0.9500 & $C(23)-C(25)$ & $1.519(10)$ \\
\hline$C(6)-C(7)$ & $1.380(9)$ & $\mathrm{C}(23)-\mathrm{H}(23)$ & 1.0000 \\
\hline $\mathrm{C}(6)-\mathrm{H}(6)$ & 0.9500 & $\mathrm{C}(24)-\mathrm{H}(24 \mathrm{~A})$ & 0.9800 \\
\hline $\mathrm{C}(7)-\mathrm{C}(8)$ & $1.386(8)$ & $\mathrm{C}(24)-\mathrm{H}(24 \mathrm{~B})$ & 0.9800 \\
\hline $\mathrm{C}(7)-\mathrm{H}(7)$ & 0.9500 & $\mathrm{C}(24)-\mathrm{H}(24 \mathrm{C})$ & 0.9800 \\
\hline $\mathrm{C}(8)-\mathrm{H}(8)$ & 0.9500 & $\mathrm{C}(25)-\mathrm{H}(25 \mathrm{~A})$ & 0.9800 \\
\hline$C(9)-C(10)$ & $1.394(7)$ & $\mathrm{C}(25)-\mathrm{H}(25 \mathrm{~B})$ & 0.9800 \\
\hline$C(9)-C(14)$ & $1.401(7)$ & $\mathrm{C}(25)-\mathrm{H}(25 \mathrm{C})$ & 0.9800 \\
\hline $\mathrm{C}(10)-\mathrm{C}(11)$ & $1.386(7)$ & $C(26)-C(28)$ & $1.507(9)$ \\
\hline $\mathrm{C}(10)-\mathrm{H}(10)$ & 0.9500 & $C(26)-C(27)$ & $1.526(9)$ \\
\hline
\end{tabular}




\begin{tabular}{|c|c|c|c|}
\hline $\mathrm{C}(26)-\mathrm{H}(26)$ & 1.0000 & $\mathrm{C}(36)-\mathrm{H}(36 \mathrm{C})$ & 0.9800 \\
\hline $\mathrm{C}(27)-\mathrm{H}(27 \mathrm{~A})$ & 0.9800 & $\mathrm{P}(3)-\mathrm{C}(57)$ & $1.826(8)$ \\
\hline $\mathrm{C}(27)-\mathrm{H}(27 \mathrm{~B})$ & 0.9800 & $\mathrm{P}(3)-\mathrm{C}(60)$ & $1.837(8)$ \\
\hline $\mathrm{C}(27)-\mathrm{H}(27 \mathrm{C})$ & 0.9800 & $\mathrm{P}(3)-\mathrm{C}(55)$ & $1.840(10)$ \\
\hline $\mathrm{C}(28)-\mathrm{H}(28 \mathrm{~A})$ & 0.9800 & $C(55)-C(56)$ & $1.449(14)$ \\
\hline $\mathrm{C}(28)-\mathrm{H}(28 \mathrm{~B})$ & 0.9800 & $\mathrm{C}(55)-\mathrm{H}(55 \mathrm{~A})$ & 0.9900 \\
\hline $\mathrm{C}(28)-\mathrm{H}(28 \mathrm{C})$ & 0.9800 & $\mathrm{C}(55)-\mathrm{H}(55 \mathrm{~B})$ & 0.9900 \\
\hline$C(29)-C(30)$ & $1.501(10)$ & $\mathrm{C}(56)-\mathrm{P}(4)$ & $1.891(12)$ \\
\hline$C(29)-C(31)$ & $1.534(8)$ & $\mathrm{C}(56)-\mathrm{H}(56 \mathrm{~A})$ & 0.9900 \\
\hline $\mathrm{C}(29)-\mathrm{H}(29)$ & 1.0000 & $\mathrm{C}(56)-\mathrm{H}(56 \mathrm{~B})$ & 0.9900 \\
\hline $\mathrm{C}(30)-\mathrm{H}(30 \mathrm{~A})$ & 0.9800 & $\mathrm{P}(4)-\mathrm{C}(63)$ & $1.826(8)$ \\
\hline $\mathrm{C}(30)-\mathrm{H}(30 \mathrm{~B})$ & 0.9800 & $\mathrm{P}(4)-\mathrm{C}(66)$ & $1.837(8)$ \\
\hline $\mathrm{C}(30)-\mathrm{H}(30 \mathrm{C})$ & 0.9800 & $C(57)-C(58)$ & $1.513(8)$ \\
\hline $\mathrm{C}(31)-\mathrm{H}(31 \mathrm{~A})$ & 0.9800 & $C(57)-C(59)$ & $1.515(8)$ \\
\hline $\mathrm{C}(31)-\mathrm{H}(31 \mathrm{~B})$ & 0.9800 & $\mathrm{C}(57)-\mathrm{H}(57)$ & 1.0000 \\
\hline $\mathrm{C}(31)-\mathrm{H}(31 \mathrm{C})$ & 0.9800 & $\mathrm{C}(58)-\mathrm{H}(58 \mathrm{~A})$ & 0.9800 \\
\hline$C(32)-C(33)$ & $1.517(10)$ & $\mathrm{C}(58)-\mathrm{H}(58 \mathrm{~B})$ & 0.9800 \\
\hline$C(32)-C(34)$ & $1.541(9)$ & $\mathrm{C}(58)-\mathrm{H}(58 \mathrm{C})$ & 0.9800 \\
\hline $\mathrm{C}(32)-\mathrm{H}(32)$ & 1.0000 & $\mathrm{C}(59)-\mathrm{H}(59 \mathrm{~A})$ & 0.9800 \\
\hline $\mathrm{C}(33)-\mathrm{H}(33 \mathrm{~A})$ & 0.9800 & $\mathrm{C}(59)-\mathrm{H}(59 \mathrm{~B})$ & 0.9800 \\
\hline C(33)-H(33B) & 0.9800 & $\mathrm{C}(59)-\mathrm{H}(59 \mathrm{C})$ & 0.9800 \\
\hline $\mathrm{C}(33)-\mathrm{H}(33 \mathrm{C})$ & 0.9800 & $\mathrm{C}(60)-\mathrm{C}(61)$ & $1.506(8)$ \\
\hline $\mathrm{C}(34)-\mathrm{H}(34 \mathrm{~A})$ & 0.9800 & $C(60)-C(62)$ & $1.522(8)$ \\
\hline $\mathrm{C}(34)-\mathrm{H}(34 \mathrm{~B})$ & 0.9800 & $\mathrm{C}(60)-\mathrm{H}(60)$ & 1.0000 \\
\hline $\mathrm{C}(34)-\mathrm{H}(34 \mathrm{C})$ & 0.9800 & $\mathrm{C}(61)-\mathrm{H}(61 \mathrm{~A})$ & 0.9800 \\
\hline $\operatorname{Pd}(2)-C(35)$ & $1.97(2)$ & $\mathrm{C}(61)-\mathrm{H}(61 \mathrm{~B})$ & 0.9800 \\
\hline $\operatorname{Pd}(2)-C\left(35^{\prime}\right)$ & 2.04(3) & $\mathrm{C}(61)-\mathrm{H}(61 \mathrm{C})$ & 0.9800 \\
\hline $\operatorname{Pd}(2)-\mathrm{N}(2)$ & $2.097(4)$ & $\mathrm{C}(62)-\mathrm{H}(62 \mathrm{~A})$ & 0.9800 \\
\hline $\operatorname{Pd}(2)-P(3)$ & $2.254(4)$ & $\mathrm{C}(62)-\mathrm{H}(62 \mathrm{~B})$ & 0.9800 \\
\hline $\mathrm{Pd}(2)-\mathrm{P}\left(3^{\prime}\right)$ & $2.273(6)$ & $\mathrm{C}(62)-\mathrm{H}(62 \mathrm{C})$ & 0.9800 \\
\hline $\mathrm{Pd}(2)-\mathrm{P}\left(4^{\prime}\right)$ & $2.317(5)$ & $\mathrm{C}(63)-\mathrm{C}(64)$ & $1.512(8)$ \\
\hline $\operatorname{Pd}(2)-P(4)$ & $2.341(3)$ & $\mathrm{C}(63)-\mathrm{C}(65)$ & $1.520(8)$ \\
\hline $\mathrm{C}(35)-\mathrm{N}(2)$ & $1.264(17)$ & $\mathrm{C}(63)-\mathrm{H}(63)$ & 1.0000 \\
\hline$C(35)-C(36)$ & $1.491(18)$ & $\mathrm{C}(64)-\mathrm{H}(64 \mathrm{~A})$ & 0.9800 \\
\hline $\mathrm{C}(36)-\mathrm{H}(36 \mathrm{~A})$ & 0.9800 & $\mathrm{C}(64)-\mathrm{H}(64 \mathrm{~B})$ & 0.9800 \\
\hline $\mathrm{C}(36)-\mathrm{H}(36 \mathrm{~B})$ & 0.9800 & $\mathrm{C}(64)-\mathrm{H}(64 \mathrm{C})$ & 0.9800 \\
\hline
\end{tabular}




\begin{tabular}{|c|c|c|c|}
\hline $\mathrm{C}(65)-\mathrm{H}(65 \mathrm{~A})$ & 0.9800 & $\mathrm{C}\left(59^{\prime}\right)-\mathrm{H}(59 \mathrm{~F})$ & 0.9800 \\
\hline $\mathrm{C}(65)-\mathrm{H}(65 \mathrm{~B})$ & 0.9800 & $C\left(60^{\prime}\right)-C\left(61^{\prime}\right)$ & $1.516(9)$ \\
\hline $\mathrm{C}(65)-\mathrm{H}(65 \mathrm{C})$ & 0.9800 & $C\left(60^{\prime}\right)-C\left(62^{\prime}\right)$ & $1.521(8)$ \\
\hline$C(66)-C(67)$ & $1.521(8)$ & $\mathrm{C}\left(60^{\prime}\right)-\mathrm{H}\left(60^{\prime}\right)$ & 1.0000 \\
\hline$C(66)-C(68)$ & $1.524(8)$ & $\mathrm{C}\left(61^{\prime}\right)-\mathrm{H}(61 \mathrm{D})$ & 0.9800 \\
\hline $\mathrm{C}(66)-\mathrm{H}(66)$ & 1.0000 & $\mathrm{C}\left(61^{\prime}\right)-\mathrm{H}(61 \mathrm{E})$ & 0.9800 \\
\hline $\mathrm{C}(67)-\mathrm{H}(67 \mathrm{~A})$ & 0.9800 & $\mathrm{C}\left(61^{\prime}\right)-\mathrm{H}(61 \mathrm{~F})$ & 0.9800 \\
\hline $\mathrm{C}(67)-\mathrm{H}(67 \mathrm{~B})$ & 0.9800 & $\mathrm{C}\left(62^{\prime}\right)-\mathrm{H}(62 \mathrm{D})$ & 0.9800 \\
\hline $\mathrm{C}(67)-\mathrm{H}(67 \mathrm{C})$ & 0.9800 & $\mathrm{C}\left(62^{\prime}\right)-\mathrm{H}(62 \mathrm{E})$ & 0.9800 \\
\hline $\mathrm{C}(68)-\mathrm{H}(68 \mathrm{~A})$ & 0.9800 & $\mathrm{C}\left(62^{\prime}\right)-\mathrm{H}(62 \mathrm{~F})$ & 0.9800 \\
\hline $\mathrm{C}(68)-\mathrm{H}(68 \mathrm{~B})$ & 0.9800 & $\mathrm{C}\left(63^{\prime}\right)-\mathrm{C}\left(65^{\prime}\right)$ & $1.518(9)$ \\
\hline $\mathrm{C}(68)-\mathrm{H}(68 \mathrm{C})$ & 0.9800 & $\mathrm{C}\left(63^{\prime}\right)-\mathrm{C}\left(64^{\prime}\right)$ & $1.522(9)$ \\
\hline $\mathrm{C}\left(35^{\prime}\right)-\mathrm{N}(2)$ & $1.16(3)$ & $\mathrm{C}\left(63^{\prime}\right)-\mathrm{H}\left(63^{\prime}\right)$ & 1.0000 \\
\hline $\mathrm{C}\left(35^{\prime}\right)-\mathrm{C}\left(36^{\prime}\right)$ & $1.53(3)$ & $\mathrm{C}\left(64^{\prime}\right)-\mathrm{H}(64 \mathrm{D})$ & 0.9800 \\
\hline $\mathrm{C}\left(36^{\prime}\right)-\mathrm{H}(36 \mathrm{D})$ & 0.9800 & $\mathrm{C}\left(64^{\prime}\right)-\mathrm{H}(64 \mathrm{E})$ & 0.9800 \\
\hline $\mathrm{C}\left(36^{\prime}\right)-\mathrm{H}(36 \mathrm{E})$ & 0.9800 & $\mathrm{C}\left(64^{\prime}\right)-\mathrm{H}(64 \mathrm{~F})$ & 0.9800 \\
\hline $\mathrm{C}\left(36^{\prime}\right)-\mathrm{H}(36 \mathrm{~F})$ & 0.9800 & $\mathrm{C}\left(65^{\prime}\right)-\mathrm{H}(65 \mathrm{D})$ & 0.9800 \\
\hline $\mathrm{P}\left(3^{\prime}\right)-\mathrm{C}\left(57^{\prime}\right)$ & $1.838(9)$ & $\mathrm{C}\left(65^{\prime}\right)-\mathrm{H}(65 \mathrm{E})$ & 0.9800 \\
\hline $\mathrm{P}\left(3^{\prime}\right)-\mathrm{C}\left(55^{\prime}\right)$ & $1.842(14)$ & $\mathrm{C}\left(65^{\prime}\right)-\mathrm{H}(65 \mathrm{~F})$ & 0.9800 \\
\hline $\mathrm{P}\left(3^{\prime}\right)-\mathrm{C}\left(60^{\prime}\right)$ & $1.844(9)$ & $\mathrm{C}\left(66^{\prime}\right)-\mathrm{C}\left(68^{\prime}\right)$ & $1.516(8)$ \\
\hline $\mathrm{C}\left(55^{\prime}\right)-\mathrm{C}\left(56^{\prime}\right)$ & $1.431(18)$ & $\mathrm{C}\left(66^{\prime}\right)-\mathrm{C}\left(67^{\prime}\right)$ & $1.518(9)$ \\
\hline $\mathrm{C}\left(55^{\prime}\right)-\mathrm{H}(55 \mathrm{C})$ & 0.9900 & $\mathrm{C}\left(66^{\prime}\right)-\mathrm{H}\left(66^{\prime}\right)$ & 1.0000 \\
\hline $\mathrm{C}\left(55^{\prime}\right)-\mathrm{H}(55 \mathrm{D})$ & 0.9900 & $\mathrm{C}\left(67^{\prime}\right)-\mathrm{H}(67 \mathrm{D})$ & 0.9800 \\
\hline $\mathrm{C}\left(56^{\prime}\right)-\mathrm{P}\left(4^{\prime}\right)$ & $1.927(16)$ & $\mathrm{C}\left(67^{\prime}\right)-\mathrm{H}(67 \mathrm{E})$ & 0.9800 \\
\hline $\mathrm{C}\left(56^{\prime}\right)-\mathrm{H}(56 \mathrm{C})$ & 0.9900 & $\mathrm{C}\left(67^{\prime}\right)-\mathrm{H}(67 \mathrm{~F})$ & 0.9800 \\
\hline $\mathrm{C}\left(56^{\prime}\right)-\mathrm{H}(56 \mathrm{D})$ & 0.9900 & $\mathrm{C}\left(68^{\prime}\right)-\mathrm{H}(68 \mathrm{D})$ & 0.9800 \\
\hline $\mathrm{P}\left(4^{\prime}\right)-\mathrm{C}\left(66^{\prime}\right)$ & $1.841(9)$ & $\mathrm{C}\left(68^{\prime}\right)-\mathrm{H}(68 \mathrm{E})$ & 0.9800 \\
\hline $\mathrm{P}\left(4^{\prime}\right)-\mathrm{C}\left(63^{\prime}\right)$ & $1.841(9)$ & $\mathrm{C}\left(68^{\prime}\right)-\mathrm{H}(68 \mathrm{~F})$ & 0.9800 \\
\hline $\mathrm{C}\left(57^{\prime}\right)-\mathrm{C}\left(58^{\prime}\right)$ & $1.517(8)$ & $\mathrm{N}(2)-\mathrm{B}(2)$ & $1.608(7)$ \\
\hline $\mathrm{C}\left(57^{\prime}\right)-\mathrm{C}\left(59^{\prime}\right)$ & $1.519(8)$ & $\mathrm{B}(2)-\mathrm{C}(49)$ & $1.618(8)$ \\
\hline $\mathrm{C}\left(57^{\prime}\right)-\mathrm{H}\left(57^{\prime}\right)$ & 1.0000 & $\mathrm{~B}(2)-\mathrm{C}(43)$ & $1.629(8)$ \\
\hline $\mathrm{C}\left(58^{\prime}\right)-\mathrm{H}(58 \mathrm{D})$ & 0.9800 & $\mathrm{~B}(2)-\mathrm{C}(37)$ & $1.644(7)$ \\
\hline $\mathrm{C}\left(58^{\prime}\right)-\mathrm{H}(58 \mathrm{E})$ & 0.9800 & $\mathrm{C}(37)-\mathrm{C}(42)$ & $1.391(8)$ \\
\hline $\mathrm{C}\left(58^{\prime}\right)-\mathrm{H}(58 \mathrm{~F})$ & 0.9800 & $\mathrm{C}(37)-\mathrm{C}(38)$ & $1.400(8)$ \\
\hline $\mathrm{C}\left(59^{\prime}\right)-\mathrm{H}(59 \mathrm{D})$ & 0.9800 & $\mathrm{C}(38)-\mathrm{C}(39)$ & $1.399(8)$ \\
\hline $\mathrm{C}\left(59^{\prime}\right)-\mathrm{H}(59 \mathrm{E})$ & 0.9800 & $\mathrm{C}(38)-\mathrm{H}(38)$ & 0.9500 \\
\hline
\end{tabular}




\begin{tabular}{|c|c|c|c|}
\hline$C(39)-C(40)$ & $1.360(9)$ & $\mathrm{C}(21)-\mathrm{P}(1)-\mathrm{C}(26)$ & $103.7(3)$ \\
\hline $\mathrm{C}(39)-\mathrm{H}(39)$ & 0.9500 & $\mathrm{C}(23)-\mathrm{P}(1)-\mathrm{C}(26)$ & $104.6(3)$ \\
\hline$C(40)-C(41)$ & $1.375(8)$ & $\mathrm{C}(21)-\mathrm{P}(1)-\mathrm{Pd}(1)$ & $107.97(18)$ \\
\hline $\mathrm{C}(40)-\mathrm{H}(40)$ & 0.9500 & $\mathrm{C}(23)-\mathrm{P}(1)-\mathrm{Pd}(1)$ & $119.8(2)$ \\
\hline$C(41)-C(42)$ & $1.407(7)$ & $\mathrm{C}(26)-\mathrm{P}(1)-\mathrm{Pd}(1)$ & $113.96(19)$ \\
\hline $\mathrm{C}(41)-\mathrm{H}(41)$ & 0.9500 & $\mathrm{C}(22)-\mathrm{P}(2)-\mathrm{C}(32)$ & $103.8(3)$ \\
\hline $\mathrm{C}(42)-\mathrm{H}(42)$ & 0.9500 & $\mathrm{C}(22)-\mathrm{P}(2)-\mathrm{C}(29)$ & $103.9(3)$ \\
\hline$C(43)-C(44)$ & $1.387(8)$ & $\mathrm{C}(32)-\mathrm{P}(2)-\mathrm{C}(29)$ & $104.6(3)$ \\
\hline$C(43)-C(48)$ & $1.400(8)$ & $\mathrm{C}(22)-\mathrm{P}(2)-\mathrm{Pd}(1)$ & $105.94(18)$ \\
\hline$C(44)-C(45)$ & $1.393(8)$ & $\mathrm{C}(32)-\mathrm{P}(2)-\mathrm{Pd}(1)$ & $122.6(2)$ \\
\hline $\mathrm{C}(44)-\mathrm{H}(44)$ & 0.9500 & $C(29)-P(2)-P d(1)$ & $114.1(2)$ \\
\hline$C(45)-C(46)$ & $1.369(9)$ & $\mathrm{C}(1)-\mathrm{N}(1)-\mathrm{B}(1)$ & $145.6(4)$ \\
\hline $\mathrm{C}(45)-\mathrm{H}(45)$ & 0.9500 & $\mathrm{C}(1)-\mathrm{N}(1)-\mathrm{Pd}(1)$ & $68.7(3)$ \\
\hline$C(46)-C(47)$ & $1.382(10)$ & $\mathrm{B}(1)-\mathrm{N}(1)-\mathrm{Pd}(1)$ & $142.7(3)$ \\
\hline $\mathrm{C}(46)-\mathrm{H}(46)$ & 0.9500 & $\mathrm{~N}(1)-\mathrm{B}(1)-\mathrm{C}(15)$ & $105.8(4)$ \\
\hline$C(47)-C(48)$ & $1.392(8)$ & $\mathrm{N}(1)-\mathrm{B}(1)-\mathrm{C}(3)$ & $107.1(4)$ \\
\hline $\mathrm{C}(47)-\mathrm{H}(47)$ & 0.9500 & $\mathrm{C}(15)-\mathrm{B}(1)-\mathrm{C}(3)$ & $113.1(4)$ \\
\hline $\mathrm{C}(48)-\mathrm{H}(48)$ & 0.9500 & $\mathrm{~N}(1)-\mathrm{B}(1)-\mathrm{C}(9)$ & $106.6(4)$ \\
\hline$C(49)-C(54)$ & $1.391(7)$ & $\mathrm{C}(15)-\mathrm{B}(1)-\mathrm{C}(9)$ & $110.9(4)$ \\
\hline$C(49)-C(50)$ & $1.410(8)$ & $\mathrm{C}(3)-\mathrm{B}(1)-\mathrm{C}(9)$ & $112.9(5)$ \\
\hline$C(50)-C(51)$ & $1.377(8)$ & $\mathrm{N}(1)-\mathrm{C}(1)-\mathrm{C}(2)$ & $137.4(5)$ \\
\hline $\mathrm{C}(50)-\mathrm{H}(50)$ & 0.9500 & $\mathrm{~N}(1)-\mathrm{C}(1)-\mathrm{Pd}(1)$ & $76.6(3)$ \\
\hline$C(51)-C(52)$ & $1.398(9)$ & $\mathrm{C}(2)-\mathrm{C}(1)-\mathrm{Pd}(1)$ & $145.9(4)$ \\
\hline $\mathrm{C}(51)-\mathrm{H}(51)$ & 0.9500 & $\mathrm{C}(1)-\mathrm{C}(2)-\mathrm{H}(2 \mathrm{~A})$ & 109.5 \\
\hline$C(52)-C(53)$ & $1.390(9)$ & $\mathrm{C}(1)-\mathrm{C}(2)-\mathrm{H}(2 \mathrm{~B})$ & 109.5 \\
\hline $\mathrm{C}(52)-\mathrm{H}(52)$ & 0.9500 & $\mathrm{H}(2 \mathrm{~A})-\mathrm{C}(2)-\mathrm{H}(2 \mathrm{~B})$ & 109.5 \\
\hline$C(53)-C(54)$ & $1.386(9)$ & $\mathrm{C}(1)-\mathrm{C}(2)-\mathrm{H}(2 \mathrm{C})$ & 109.5 \\
\hline $\mathrm{C}(53)-\mathrm{H}(53)$ & 0.9500 & $\mathrm{H}(2 \mathrm{~A})-\mathrm{C}(2)-\mathrm{H}(2 \mathrm{C})$ & 109.5 \\
\hline $\mathrm{C}(54)-\mathrm{H}(54)$ & 0.9500 & $\mathrm{H}(2 \mathrm{~B})-\mathrm{C}(2)-\mathrm{H}(2 \mathrm{C})$ & 109.5 \\
\hline $\mathrm{C}(1)-\mathrm{Pd}(1)-\mathrm{N}(1)$ & $34.64(18)$ & $\mathrm{C}(8)-\mathrm{C}(3)-\mathrm{C}(4)$ & $115.6(5)$ \\
\hline$C(1)-P d(1)-P(1)$ & $115.00(15)$ & $\mathrm{C}(8)-\mathrm{C}(3)-\mathrm{B}(1)$ & $123.1(4)$ \\
\hline $\mathrm{N}(1)-\mathrm{Pd}(1)-\mathrm{P}(1)$ & $148.97(11)$ & $\mathrm{C}(4)-\mathrm{C}(3)-\mathrm{B}(1)$ & $121.2(5)$ \\
\hline$C(1)-P d(1)-P(2)$ & $156.94(15)$ & $C(5)-C(4)-C(3)$ & $122.4(6)$ \\
\hline $\mathrm{N}(1)-\mathrm{Pd}(1)-\mathrm{P}(2)$ & $122.56(11)$ & $\mathrm{C}(5)-\mathrm{C}(4)-\mathrm{H}(4)$ & 118.8 \\
\hline $\mathrm{P}(1)-\mathrm{Pd}(1)-\mathrm{P}(2)$ & $88.05(5)$ & $\mathrm{C}(3)-\mathrm{C}(4)-\mathrm{H}(4)$ & 118.8 \\
\hline $\mathrm{C}(21)-\mathrm{P}(1)-\mathrm{C}(23)$ & $105.4(3)$ & $C(6)-C(5)-C(4)$ & $119.5(5)$ \\
\hline
\end{tabular}




\begin{tabular}{|c|c|c|c|}
\hline $\mathrm{C}(6)-\mathrm{C}(5)-\mathrm{H}(5)$ & 120.2 & $\mathrm{C}(16)-\mathrm{C}(17)-\mathrm{H}(17)$ & 119.5 \\
\hline $\mathrm{C}(4)-\mathrm{C}(5)-\mathrm{H}(5)$ & 120.2 & $\mathrm{C}(18)-\mathrm{C}(17)-\mathrm{H}(17)$ & 119.5 \\
\hline$C(7)-C(6)-C(5)$ & $119.3(6)$ & $C(19)-C(18)-C(17)$ & $118.3(5)$ \\
\hline $\mathrm{C}(7)-\mathrm{C}(6)-\mathrm{H}(6)$ & 120.3 & $\mathrm{C}(19)-\mathrm{C}(18)-\mathrm{H}(18)$ & 120.8 \\
\hline $\mathrm{C}(5)-\mathrm{C}(6)-\mathrm{H}(6)$ & 120.3 & $\mathrm{C}(17)-\mathrm{C}(18)-\mathrm{H}(18)$ & 120.8 \\
\hline$C(6)-C(7)-C(8)$ & $120.5(6)$ & $C(20)-C(19)-C(18)$ & $120.1(5)$ \\
\hline $\mathrm{C}(6)-\mathrm{C}(7)-\mathrm{H}(7)$ & 119.8 & $\mathrm{C}(20)-\mathrm{C}(19)-\mathrm{H}(19)$ & 120.0 \\
\hline $\mathrm{C}(8)-\mathrm{C}(7)-\mathrm{H}(7)$ & 119.8 & C(18)-C(19)-H(19) & 120.0 \\
\hline$C(7)-C(8)-C(3)$ & $122.6(5)$ & $C(19)-C(20)-C(15)$ & $123.2(5)$ \\
\hline $\mathrm{C}(7)-\mathrm{C}(8)-\mathrm{H}(8)$ & 118.7 & $\mathrm{C}(19)-\mathrm{C}(20)-\mathrm{H}(20)$ & 118.4 \\
\hline $\mathrm{C}(3)-\mathrm{C}(8)-\mathrm{H}(8)$ & 118.7 & $\mathrm{C}(15)-\mathrm{C}(20)-\mathrm{H}(20)$ & 118.4 \\
\hline$C(10)-C(9)-C(14)$ & $115.2(4)$ & $\mathrm{C}(22)-\mathrm{C}(21)-\mathrm{P}(1)$ & $112.9(4)$ \\
\hline $\mathrm{C}(10)-\mathrm{C}(9)-\mathrm{B}(1)$ & $122.0(4)$ & $\mathrm{C}(22)-\mathrm{C}(21)-\mathrm{H}(21 \mathrm{~A})$ & 109.0 \\
\hline $\mathrm{C}(14)-\mathrm{C}(9)-\mathrm{B}(1)$ & $122.7(5)$ & $\mathrm{P}(1)-\mathrm{C}(21)-\mathrm{H}(21 \mathrm{~A})$ & 109.0 \\
\hline$C(11)-C(10)-C(9)$ & $122.8(5)$ & $\mathrm{C}(22)-\mathrm{C}(21)-\mathrm{H}(21 \mathrm{~B})$ & 109.0 \\
\hline $\mathrm{C}(11)-\mathrm{C}(10)-\mathrm{H}(10)$ & 118.6 & $\mathrm{P}(1)-\mathrm{C}(21)-\mathrm{H}(21 \mathrm{~B})$ & 109.0 \\
\hline $\mathrm{C}(9)-\mathrm{C}(10)-\mathrm{H}(10)$ & 118.6 & $\mathrm{H}(21 \mathrm{~A})-\mathrm{C}(21)-\mathrm{H}(21 \mathrm{~B})$ & 107.8 \\
\hline $\mathrm{C}(12)-\mathrm{C}(11)-\mathrm{C}(10)$ & $120.3(5)$ & $\mathrm{C}(21)-\mathrm{C}(22)-\mathrm{P}(2)$ & $112.8(4)$ \\
\hline $\mathrm{C}(12)-\mathrm{C}(11)-\mathrm{H}(11)$ & 119.9 & $\mathrm{C}(21)-\mathrm{C}(22)-\mathrm{H}(22 \mathrm{~A})$ & 109.0 \\
\hline $\mathrm{C}(10)-\mathrm{C}(11)-\mathrm{H}(11)$ & 119.9 & $\mathrm{P}(2)-\mathrm{C}(22)-\mathrm{H}(22 \mathrm{~A})$ & 109.0 \\
\hline $\mathrm{C}(13)-\mathrm{C}(12)-\mathrm{C}(11)$ & $118.8(5)$ & $\mathrm{C}(21)-\mathrm{C}(22)-\mathrm{H}(22 \mathrm{~B})$ & 109.0 \\
\hline $\mathrm{C}(13)-\mathrm{C}(12)-\mathrm{H}(12)$ & 120.6 & $\mathrm{P}(2)-\mathrm{C}(22)-\mathrm{H}(22 \mathrm{~B})$ & 109.0 \\
\hline $\mathrm{C}(11)-\mathrm{C}(12)-\mathrm{H}(12)$ & 120.6 & $\mathrm{H}(22 \mathrm{~A})-\mathrm{C}(22)-\mathrm{H}(22 \mathrm{~B})$ & 107.8 \\
\hline $\mathrm{C}(12)-\mathrm{C}(13)-\mathrm{C}(14)$ & $120.6(6)$ & $\mathrm{C}(24)-\mathrm{C}(23)-\mathrm{C}(25)$ & $110.5(6)$ \\
\hline $\mathrm{C}(12)-\mathrm{C}(13)-\mathrm{H}(13)$ & 119.7 & $\mathrm{C}(24)-\mathrm{C}(23)-\mathrm{P}(1)$ & $110.7(4)$ \\
\hline $\mathrm{C}(14)-\mathrm{C}(13)-\mathrm{H}(13)$ & 119.7 & $\mathrm{C}(25)-\mathrm{C}(23)-\mathrm{P}(1)$ & 109.3(4) \\
\hline $\mathrm{C}(13)-\mathrm{C}(14)-\mathrm{C}(9)$ & $122.2(5)$ & $\mathrm{C}(24)-\mathrm{C}(23)-\mathrm{H}(23)$ & 108.7 \\
\hline $\mathrm{C}(13)-\mathrm{C}(14)-\mathrm{H}(14)$ & 118.9 & $\mathrm{C}(25)-\mathrm{C}(23)-\mathrm{H}(23)$ & 108.7 \\
\hline $\mathrm{C}(9)-\mathrm{C}(14)-\mathrm{H}(14)$ & 118.9 & $\mathrm{P}(1)-\mathrm{C}(23)-\mathrm{H}(23)$ & 108.7 \\
\hline $\mathrm{C}(16)-\mathrm{C}(15)-\mathrm{C}(20)$ & $114.9(5)$ & $\mathrm{C}(23)-\mathrm{C}(24)-\mathrm{H}(24 \mathrm{~A})$ & 109.5 \\
\hline $\mathrm{C}(16)-\mathrm{C}(15)-\mathrm{B}(1)$ & $124.3(5)$ & $\mathrm{C}(23)-\mathrm{C}(24)-\mathrm{H}(24 \mathrm{~B})$ & 109.5 \\
\hline $\mathrm{C}(20)-\mathrm{C}(15)-\mathrm{B}(1)$ & $120.7(5)$ & $\mathrm{H}(24 \mathrm{~A})-\mathrm{C}(24)-\mathrm{H}(24 \mathrm{~B})$ & 109.5 \\
\hline $\mathrm{C}(17)-\mathrm{C}(16)-\mathrm{C}(15)$ & $122.5(5)$ & $\mathrm{C}(23)-\mathrm{C}(24)-\mathrm{H}(24 \mathrm{C})$ & 109.5 \\
\hline $\mathrm{C}(17)-\mathrm{C}(16)-\mathrm{H}(16)$ & 118.7 & $\mathrm{H}(24 \mathrm{~A})-\mathrm{C}(24)-\mathrm{H}(24 \mathrm{C})$ & 109.5 \\
\hline $\mathrm{C}(15)-\mathrm{C}(16)-\mathrm{H}(16)$ & 118.7 & $\mathrm{H}(24 \mathrm{~B})-\mathrm{C}(24)-\mathrm{H}(24 \mathrm{C})$ & 109.5 \\
\hline$C(16)-C(17)-C(18)$ & $121.0(6)$ & $\mathrm{C}(23)-\mathrm{C}(25)-\mathrm{H}(25 \mathrm{~A})$ & 109.5 \\
\hline
\end{tabular}




\begin{tabular}{|c|c|c|c|}
\hline $\mathrm{C}(23)-\mathrm{C}(25)-\mathrm{H}(25 \mathrm{~B})$ & 109.5 & $\mathrm{C}(29)-\mathrm{C}(31)-\mathrm{H}(31 \mathrm{~B})$ & 109.5 \\
\hline $\mathrm{H}(25 \mathrm{~A})-\mathrm{C}(25)-\mathrm{H}(25 \mathrm{~B})$ & 109.5 & $\mathrm{H}(31 \mathrm{~A})-\mathrm{C}(31)-\mathrm{H}(31 \mathrm{~B})$ & 109.5 \\
\hline $\mathrm{C}(23)-\mathrm{C}(25)-\mathrm{H}(25 \mathrm{C})$ & 109.5 & $\mathrm{C}(29)-\mathrm{C}(31)-\mathrm{H}(31 \mathrm{C})$ & 109.5 \\
\hline $\mathrm{H}(25 \mathrm{~A})-\mathrm{C}(25)-\mathrm{H}(25 \mathrm{C})$ & 109.5 & $\mathrm{H}(31 \mathrm{~A})-\mathrm{C}(31)-\mathrm{H}(31 \mathrm{C})$ & 109.5 \\
\hline $\mathrm{H}(25 \mathrm{~B})-\mathrm{C}(25)-\mathrm{H}(25 \mathrm{C})$ & 109.5 & $\mathrm{H}(31 \mathrm{~B})-\mathrm{C}(31)-\mathrm{H}(31 \mathrm{C})$ & 109.5 \\
\hline $\mathrm{C}(28)-\mathrm{C}(26)-\mathrm{C}(27)$ & $111.4(6)$ & $\mathrm{C}(33)-\mathrm{C}(32)-\mathrm{C}(34)$ & $110.7(6)$ \\
\hline $\mathrm{C}(28)-\mathrm{C}(26)-\mathrm{P}(1)$ & $114.5(4)$ & $\mathrm{C}(33)-\mathrm{C}(32)-\mathrm{P}(2)$ & $110.6(4)$ \\
\hline $\mathrm{C}(27)-\mathrm{C}(26)-\mathrm{P}(1)$ & 111.2(4) & $\mathrm{C}(34)-\mathrm{C}(32)-\mathrm{P}(2)$ & $110.4(4)$ \\
\hline $\mathrm{C}(28)-\mathrm{C}(26)-\mathrm{H}(26)$ & 106.4 & $\mathrm{C}(33)-\mathrm{C}(32)-\mathrm{H}(32)$ & 108.4 \\
\hline $\mathrm{C}(27)-\mathrm{C}(26)-\mathrm{H}(26)$ & 106.4 & $\mathrm{C}(34)-\mathrm{C}(32)-\mathrm{H}(32)$ & 108.4 \\
\hline $\mathrm{P}(1)-\mathrm{C}(26)-\mathrm{H}(26)$ & 106.4 & $\mathrm{P}(2)-\mathrm{C}(32)-\mathrm{H}(32)$ & 108.4 \\
\hline $\mathrm{C}(26)-\mathrm{C}(27)-\mathrm{H}(27 \mathrm{~A})$ & 109.5 & $\mathrm{C}(32)-\mathrm{C}(33)-\mathrm{H}(33 \mathrm{~A})$ & 109.5 \\
\hline $\mathrm{C}(26)-\mathrm{C}(27)-\mathrm{H}(27 \mathrm{~B})$ & 109.5 & $\mathrm{C}(32)-\mathrm{C}(33)-\mathrm{H}(33 \mathrm{~B})$ & 109.5 \\
\hline $\mathrm{H}(27 \mathrm{~A})-\mathrm{C}(27)-\mathrm{H}(27 \mathrm{~B})$ & 109.5 & $\mathrm{H}(33 \mathrm{~A})-\mathrm{C}(33)-\mathrm{H}(33 \mathrm{~B})$ & 109.5 \\
\hline $\mathrm{C}(26)-\mathrm{C}(27)-\mathrm{H}(27 \mathrm{C})$ & 109.5 & $\mathrm{C}(32)-\mathrm{C}(33)-\mathrm{H}(33 \mathrm{C})$ & 109.5 \\
\hline $\mathrm{H}(27 \mathrm{~A})-\mathrm{C}(27)-\mathrm{H}(27 \mathrm{C})$ & 109.5 & $\mathrm{H}(33 \mathrm{~A})-\mathrm{C}(33)-\mathrm{H}(33 \mathrm{C})$ & 109.5 \\
\hline $\mathrm{H}(27 \mathrm{~B})-\mathrm{C}(27)-\mathrm{H}(27 \mathrm{C})$ & 109.5 & $\mathrm{H}(33 \mathrm{~B})-\mathrm{C}(33)-\mathrm{H}(33 \mathrm{C})$ & 109.5 \\
\hline $\mathrm{C}(26)-\mathrm{C}(28)-\mathrm{H}(28 \mathrm{~A})$ & 109.5 & $\mathrm{C}(32)-\mathrm{C}(34)-\mathrm{H}(34 \mathrm{~A})$ & 109.5 \\
\hline $\mathrm{C}(26)-\mathrm{C}(28)-\mathrm{H}(28 \mathrm{~B})$ & 109.5 & $\mathrm{C}(32)-\mathrm{C}(34)-\mathrm{H}(34 \mathrm{~B})$ & 109.5 \\
\hline $\mathrm{H}(28 \mathrm{~A})-\mathrm{C}(28)-\mathrm{H}(28 \mathrm{~B})$ & 109.5 & $\mathrm{H}(34 \mathrm{~A})-\mathrm{C}(34)-\mathrm{H}(34 \mathrm{~B})$ & 109.5 \\
\hline $\mathrm{C}(26)-\mathrm{C}(28)-\mathrm{H}(28 \mathrm{C})$ & 109.5 & $\mathrm{C}(32)-\mathrm{C}(34)-\mathrm{H}(34 \mathrm{C})$ & 109.5 \\
\hline $\mathrm{H}(28 \mathrm{~A})-\mathrm{C}(28)-\mathrm{H}(28 \mathrm{C})$ & 109.5 & $\mathrm{H}(34 \mathrm{~A})-\mathrm{C}(34)-\mathrm{H}(34 \mathrm{C})$ & 109.5 \\
\hline $\mathrm{H}(28 \mathrm{~B})-\mathrm{C}(28)-\mathrm{H}(28 \mathrm{C})$ & 109.5 & $\mathrm{H}(34 \mathrm{~B})-\mathrm{C}(34)-\mathrm{H}(34 \mathrm{C})$ & 109.5 \\
\hline $\mathrm{C}(30)-\mathrm{C}(29)-\mathrm{C}(31)$ & $112.0(6)$ & $\mathrm{C}(35)-\mathrm{Pd}(2)-\mathrm{N}(2)$ & $36.1(5)$ \\
\hline $\mathrm{C}(30)-\mathrm{C}(29)-\mathrm{P}(2)$ & $111.2(4)$ & $\mathrm{C}\left(35^{\prime}\right)-\mathrm{Pd}(2)-\mathrm{N}(2)$ & $32.5(8)$ \\
\hline $\mathrm{C}(31)-\mathrm{C}(29)-\mathrm{P}(2)$ & $114.0(5)$ & $\mathrm{C}(35)-\operatorname{Pd}(2)-\mathrm{P}(3)$ & $111.3(5)$ \\
\hline $\mathrm{C}(30)-\mathrm{C}(29)-\mathrm{H}(29)$ & 106.4 & $\mathrm{~N}(2)-\mathrm{Pd}(2)-\mathrm{P}(3)$ & $147.2(3)$ \\
\hline $\mathrm{C}(31)-\mathrm{C}(29)-\mathrm{H}(29)$ & 106.4 & $\mathrm{C}\left(35^{\prime}\right)-\mathrm{Pd}(2)-\mathrm{P}\left(3^{\prime}\right)$ & $118.8(9)$ \\
\hline $\mathrm{P}(2)-\mathrm{C}(29)-\mathrm{H}(29)$ & 106.4 & $\mathrm{~N}(2)-\mathrm{Pd}(2)-\mathrm{P}\left(3^{\prime}\right)$ & $151.1(4)$ \\
\hline $\mathrm{C}(29)-\mathrm{C}(30)-\mathrm{H}(30 \mathrm{~A})$ & 109.5 & $\mathrm{C}\left(35^{\prime}\right)-\mathrm{Pd}(2)-\mathrm{P}\left(4^{\prime}\right)$ & $151.4(8)$ \\
\hline $\mathrm{C}(29)-\mathrm{C}(30)-\mathrm{H}(30 \mathrm{~B})$ & 109.5 & $N(2)-P d(2)-P\left(4^{\prime}\right)$ & $119.0(2)$ \\
\hline $\mathrm{H}(30 \mathrm{~A})-\mathrm{C}(30)-\mathrm{H}(30 \mathrm{~B})$ & 109.5 & $\mathrm{P}\left(3^{\prime}\right)-\mathrm{Pd}(2)-\mathrm{P}\left(4^{\prime}\right)$ & $89.2(5)$ \\
\hline $\mathrm{C}(29)-\mathrm{C}(30)-\mathrm{H}(30 \mathrm{C})$ & 109.5 & $\mathrm{C}(35)-\mathrm{Pd}(2)-\mathrm{P}(4)$ & $162.8(5)$ \\
\hline $\mathrm{H}(30 \mathrm{~A})-\mathrm{C}(30)-\mathrm{H}(30 \mathrm{C})$ & 109.5 & $\mathrm{~N}(2)-\mathrm{Pd}(2)-\mathrm{P}(4)$ & $127.42(16)$ \\
\hline $\mathrm{H}(30 \mathrm{~B})-\mathrm{C}(30)-\mathrm{H}(30 \mathrm{C})$ & 109.5 & $\mathrm{P}(3)-\mathrm{Pd}(2)-\mathrm{P}(4)$ & $85.4(3)$ \\
\hline $\mathrm{C}(29)-\mathrm{C}(31)-\mathrm{H}(31 \mathrm{~A})$ & 109.5 & $\mathrm{~N}(2)-\mathrm{C}(35)-\mathrm{C}(36)$ & $134.8(14)$ \\
\hline
\end{tabular}




\begin{tabular}{|c|c|c|c|}
\hline $\mathrm{N}(2)-\mathrm{C}(35)-\mathrm{Pd}(2)$ & $77.6(10)$ & $\mathrm{C}(59)-\mathrm{C}(57)-\mathrm{H}(57)$ & 109.3 \\
\hline $\mathrm{C}(36)-\mathrm{C}(35)-\mathrm{Pd}(2)$ & $147.6(10)$ & $\mathrm{P}(3)-\mathrm{C}(57)-\mathrm{H}(57)$ & 109.3 \\
\hline $\mathrm{C}(35)-\mathrm{C}(36)-\mathrm{H}(36 \mathrm{~A})$ & 109.5 & $\mathrm{C}(57)-\mathrm{C}(58)-\mathrm{H}(58 \mathrm{~A})$ & 109.5 \\
\hline $\mathrm{C}(35)-\mathrm{C}(36)-\mathrm{H}(36 \mathrm{~B})$ & 109.5 & $\mathrm{C}(57)-\mathrm{C}(58)-\mathrm{H}(58 \mathrm{~B})$ & 109.5 \\
\hline $\mathrm{H}(36 \mathrm{~A})-\mathrm{C}(36)-\mathrm{H}(36 \mathrm{~B})$ & 109.5 & $\mathrm{H}(58 \mathrm{~A})-\mathrm{C}(58)-\mathrm{H}(58 \mathrm{~B})$ & 109.5 \\
\hline $\mathrm{C}(35)-\mathrm{C}(36)-\mathrm{H}(36 \mathrm{C})$ & 109.5 & $\mathrm{C}(57)-\mathrm{C}(58)-\mathrm{H}(58 \mathrm{C})$ & 109.5 \\
\hline $\mathrm{H}(36 \mathrm{~A})-\mathrm{C}(36)-\mathrm{H}(36 \mathrm{C})$ & 109.5 & $\mathrm{H}(58 \mathrm{~A})-\mathrm{C}(58)-\mathrm{H}(58 \mathrm{C})$ & 109.5 \\
\hline $\mathrm{H}(36 \mathrm{~B})-\mathrm{C}(36)-\mathrm{H}(36 \mathrm{C})$ & 109.5 & $\mathrm{H}(58 \mathrm{~B})-\mathrm{C}(58)-\mathrm{H}(58 \mathrm{C})$ & 109.5 \\
\hline $\mathrm{C}(57)-\mathrm{P}(3)-\mathrm{C}(60)$ & $90.6(6)$ & $\mathrm{C}(57)-\mathrm{C}(59)-\mathrm{H}(59 \mathrm{~A})$ & 109.5 \\
\hline $\mathrm{C}(57)-\mathrm{P}(3)-\mathrm{C}(55)$ & $107.5(8)$ & $\mathrm{C}(57)-\mathrm{C}(59)-\mathrm{H}(59 \mathrm{~B})$ & 109.5 \\
\hline $\mathrm{C}(60)-\mathrm{P}(3)-\mathrm{C}(55)$ & $103.4(6)$ & H(59A)-C(59)-H(59B) & 109.5 \\
\hline $\mathrm{C}(57)-\mathrm{P}(3)-\mathrm{Pd}(2)$ & $118.9(7)$ & $\mathrm{C}(57)-\mathrm{C}(59)-\mathrm{H}(59 \mathrm{C})$ & 109.5 \\
\hline$C(60)-P(3)-P d(2)$ & $122.8(5)$ & $\mathrm{H}(59 \mathrm{~A})-\mathrm{C}(59)-\mathrm{H}(59 \mathrm{C})$ & 109.5 \\
\hline$C(55)-P(3)-P d(2)$ & $111.0(6)$ & H(59B)-C(59)-H(59C) & 109.5 \\
\hline $\mathrm{C}(56)-\mathrm{C}(55)-\mathrm{P}(3)$ & $113.8(9)$ & $C(61)-C(60)-C(62)$ & $114.3(12)$ \\
\hline $\mathrm{C}(56)-\mathrm{C}(55)-\mathrm{H}(55 \mathrm{~A})$ & 108.8 & $\mathrm{C}(61)-\mathrm{C}(60)-\mathrm{P}(3)$ & $107.1(8)$ \\
\hline $\mathrm{P}(3)-\mathrm{C}(55)-\mathrm{H}(55 \mathrm{~A})$ & 108.8 & $\mathrm{C}(62)-\mathrm{C}(60)-\mathrm{P}(3)$ & $116.6(10)$ \\
\hline $\mathrm{C}(56)-\mathrm{C}(55)-\mathrm{H}(55 \mathrm{~B})$ & 108.8 & $\mathrm{C}(61)-\mathrm{C}(60)-\mathrm{H}(60)$ & 106.0 \\
\hline $\mathrm{P}(3)-\mathrm{C}(55)-\mathrm{H}(55 \mathrm{~B})$ & 108.8 & $\mathrm{C}(62)-\mathrm{C}(60)-\mathrm{H}(60)$ & 106.0 \\
\hline $\mathrm{H}(55 \mathrm{~A})-\mathrm{C}(55)-\mathrm{H}(55 \mathrm{~B})$ & 107.7 & $\mathrm{P}(3)-\mathrm{C}(60)-\mathrm{H}(60)$ & 106.0 \\
\hline $\mathrm{C}(55)-\mathrm{C}(56)-\mathrm{P}(4)$ & $115.8(8)$ & $\mathrm{C}(60)-\mathrm{C}(61)-\mathrm{H}(61 \mathrm{~A})$ & 109.5 \\
\hline $\mathrm{C}(55)-\mathrm{C}(56)-\mathrm{H}(56 \mathrm{~A})$ & 108.3 & $\mathrm{C}(60)-\mathrm{C}(61)-\mathrm{H}(61 \mathrm{~B})$ & 109.5 \\
\hline $\mathrm{P}(4)-\mathrm{C}(56)-\mathrm{H}(56 \mathrm{~A})$ & 108.3 & $\mathrm{H}(61 \mathrm{~A})-\mathrm{C}(61)-\mathrm{H}(61 \mathrm{~B})$ & 109.5 \\
\hline $\mathrm{C}(55)-\mathrm{C}(56)-\mathrm{H}(56 \mathrm{~B})$ & 108.3 & $\mathrm{C}(60)-\mathrm{C}(61)-\mathrm{H}(61 \mathrm{C})$ & 109.5 \\
\hline $\mathrm{P}(4)-\mathrm{C}(56)-\mathrm{H}(56 \mathrm{~B})$ & 108.3 & $\mathrm{H}(61 \mathrm{~A})-\mathrm{C}(61)-\mathrm{H}(61 \mathrm{C})$ & 109.5 \\
\hline $\mathrm{H}(56 \mathrm{~A})-\mathrm{C}(56)-\mathrm{H}(56 \mathrm{~B})$ & 107.4 & $\mathrm{H}(61 \mathrm{~B})-\mathrm{C}(61)-\mathrm{H}(61 \mathrm{C})$ & 109.5 \\
\hline$C(63)-P(4)-C(66)$ & $105.5(7)$ & $\mathrm{C}(60)-\mathrm{C}(62)-\mathrm{H}(62 \mathrm{~A})$ & 109.5 \\
\hline$C(63)-P(4)-C(56)$ & $104.7(7)$ & $\mathrm{C}(60)-\mathrm{C}(62)-\mathrm{H}(62 \mathrm{~B})$ & 109.5 \\
\hline$C(66)-P(4)-C(56)$ & $100.1(8)$ & $\mathrm{H}(62 \mathrm{~A})-\mathrm{C}(62)-\mathrm{H}(62 \mathrm{~B})$ & 109.5 \\
\hline$C(63)-P(4)-P d(2)$ & $121.5(4)$ & $\mathrm{C}(60)-\mathrm{C}(62)-\mathrm{H}(62 \mathrm{C})$ & 109.5 \\
\hline$C(66)-P(4)-P d(2)$ & $114.9(5)$ & $H(62 A)-C(62)-H(62 C)$ & 109.5 \\
\hline$C(56)-P(4)-P d(2)$ & $107.4(4)$ & $\mathrm{H}(62 \mathrm{~B})-\mathrm{C}(62)-\mathrm{H}(62 \mathrm{C})$ & 109.5 \\
\hline $\mathrm{C}(58)-\mathrm{C}(57)-\mathrm{C}(59)$ & $114.4(11)$ & $C(64)-C(63)-C(65)$ & $111.1(13)$ \\
\hline $\mathrm{C}(58)-\mathrm{C}(57)-\mathrm{P}(3)$ & $109.3(8)$ & $\mathrm{C}(64)-\mathrm{C}(63)-\mathrm{P}(4)$ & $112.2(8)$ \\
\hline $\mathrm{C}(59)-\mathrm{C}(57)-\mathrm{P}(3)$ & $105.2(11)$ & $\mathrm{C}(65)-\mathrm{C}(63)-\mathrm{P}(4)$ & $115.4(9)$ \\
\hline $\mathrm{C}(58)-\mathrm{C}(57)-\mathrm{H}(57)$ & 109.3 & $\mathrm{C}(64)-\mathrm{C}(63)-\mathrm{H}(63)$ & 105.8 \\
\hline
\end{tabular}




\begin{tabular}{|c|c|c|c|}
\hline $\mathrm{C}(65)-\mathrm{C}(63)-\mathrm{H}(63)$ & 105.8 & $\mathrm{C}\left(35^{\prime}\right)-\mathrm{C}\left(36^{\prime}\right)-\mathrm{H}(36 \mathrm{E})$ & 109.5 \\
\hline $\mathrm{P}(4)-\mathrm{C}(63)-\mathrm{H}(63)$ & 105.8 & $\mathrm{H}(36 \mathrm{D})-\mathrm{C}\left(36^{\prime}\right)-\mathrm{H}(36 \mathrm{E})$ & 109.5 \\
\hline $\mathrm{C}(63)-\mathrm{C}(64)-\mathrm{H}(64 \mathrm{~A})$ & 109.5 & $\mathrm{C}\left(35^{\prime}\right)-\mathrm{C}\left(36^{\prime}\right)-\mathrm{H}(36 \mathrm{~F})$ & 109.5 \\
\hline $\mathrm{C}(63)-\mathrm{C}(64)-\mathrm{H}(64 \mathrm{~B})$ & 109.5 & $\mathrm{H}(36 \mathrm{D})-\mathrm{C}\left(36^{\prime}\right)-\mathrm{H}(36 \mathrm{~F})$ & 109.5 \\
\hline $\mathrm{H}(64 \mathrm{~A})-\mathrm{C}(64)-\mathrm{H}(64 \mathrm{~B})$ & 109.5 & $\mathrm{H}(36 \mathrm{E})-\mathrm{C}\left(36^{\prime}\right)-\mathrm{H}(36 \mathrm{~F})$ & 109.5 \\
\hline $\mathrm{C}(63)-\mathrm{C}(64)-\mathrm{H}(64 \mathrm{C})$ & 109.5 & $\mathrm{C}\left(57^{\prime}\right)-\mathrm{P}\left(3^{\prime}\right)-\mathrm{C}\left(55^{\prime}\right)$ & $105.0(14)$ \\
\hline $\mathrm{H}(64 \mathrm{~A})-\mathrm{C}(64)-\mathrm{H}(64 \mathrm{C})$ & 109.5 & $\mathrm{C}\left(57^{\prime}\right)-\mathrm{P}\left(3^{\prime}\right)-\mathrm{C}\left(60^{\prime}\right)$ & $120.7(10)$ \\
\hline H(64B)-C(64)-H(64C) & 109.5 & $\mathrm{C}\left(55^{\prime}\right)-\mathrm{P}\left(3^{\prime}\right)-\mathrm{C}\left(60^{\prime}\right)$ & $103.8(12)$ \\
\hline $\mathrm{C}(63)-\mathrm{C}(65)-\mathrm{H}(65 \mathrm{~A})$ & 109.5 & $\mathrm{C}\left(57^{\prime}\right)-\mathrm{P}\left(3^{\prime}\right)-\mathrm{Pd}(2)$ & 109.0(11) \\
\hline $\mathrm{C}(63)-\mathrm{C}(65)-\mathrm{H}(65 \mathrm{~B})$ & 109.5 & $\mathrm{C}\left(55^{\prime}\right)-\mathrm{P}\left(3^{\prime}\right)-\mathrm{Pd}(2)$ & $104.9(8)$ \\
\hline $\mathrm{H}(65 \mathrm{~A})-\mathrm{C}(65)-\mathrm{H}(65 \mathrm{~B})$ & 109.5 & $\mathrm{C}\left(60^{\prime}\right)-\mathrm{P}\left(3^{\prime}\right)-\mathrm{Pd}(2)$ & $112.0(8)$ \\
\hline $\mathrm{C}(63)-\mathrm{C}(65)-\mathrm{H}(65 \mathrm{C})$ & 109.5 & $\mathrm{C}\left(56^{\prime}\right)-\mathrm{C}\left(55^{\prime}\right)-\mathrm{P}\left(3^{\prime}\right)$ & $115.9(14)$ \\
\hline $\mathrm{H}(65 \mathrm{~A})-\mathrm{C}(65)-\mathrm{H}(65 \mathrm{C})$ & 109.5 & $\mathrm{C}\left(56^{\prime}\right)-\mathrm{C}\left(55^{\prime}\right)-\mathrm{H}(55 \mathrm{C})$ & 108.3 \\
\hline $\mathrm{H}(65 \mathrm{~B})-\mathrm{C}(65)-\mathrm{H}(65 \mathrm{C})$ & 109.5 & $\mathrm{P}\left(3^{\prime}\right)-\mathrm{C}\left(55^{\prime}\right)-\mathrm{H}(55 \mathrm{C})$ & 108.3 \\
\hline $\mathrm{C}(67)-\mathrm{C}(66)-\mathrm{C}(68)$ & $112.8(17)$ & $\mathrm{C}\left(56^{\prime}\right)-\mathrm{C}\left(55^{\prime}\right)-\mathrm{H}(55 \mathrm{D})$ & 108.3 \\
\hline $\mathrm{C}(67)-\mathrm{C}(66)-\mathrm{P}(4)$ & $110.7(15)$ & $\mathrm{P}\left(3^{\prime}\right)-\mathrm{C}\left(55^{\prime}\right)-\mathrm{H}(55 \mathrm{D})$ & 108.3 \\
\hline $\mathrm{C}(68)-\mathrm{C}(66)-\mathrm{P}(4)$ & $110.0(9)$ & $\mathrm{H}(55 \mathrm{C})-\mathrm{C}\left(55^{\prime}\right)-\mathrm{H}(55 \mathrm{D})$ & 107.4 \\
\hline $\mathrm{C}(67)-\mathrm{C}(66)-\mathrm{H}(66)$ & 107.7 & $\mathrm{C}\left(55^{\prime}\right)-\mathrm{C}\left(56^{\prime}\right)-\mathrm{P}\left(4^{\prime}\right)$ & $110.3(14)$ \\
\hline $\mathrm{C}(68)-\mathrm{C}(66)-\mathrm{H}(66)$ & 107.7 & $\mathrm{C}\left(55^{\prime}\right)-\mathrm{C}\left(56^{\prime}\right)-\mathrm{H}(56 \mathrm{C})$ & 109.6 \\
\hline $\mathrm{P}(4)-\mathrm{C}(66)-\mathrm{H}(66)$ & 107.7 & $\mathrm{P}\left(4^{\prime}\right)-\mathrm{C}\left(56^{\prime}\right)-\mathrm{H}(56 \mathrm{C})$ & 109.6 \\
\hline $\mathrm{C}(66)-\mathrm{C}(67)-\mathrm{H}(67 \mathrm{~A})$ & 109.5 & $\mathrm{C}\left(55^{\prime}\right)-\mathrm{C}\left(56^{\prime}\right)-\mathrm{H}(56 \mathrm{D})$ & 109.6 \\
\hline $\mathrm{C}(66)-\mathrm{C}(67)-\mathrm{H}(67 \mathrm{~B})$ & 109.5 & $\mathrm{P}\left(4^{\prime}\right)-\mathrm{C}\left(56^{\prime}\right)-\mathrm{H}(56 \mathrm{D})$ & 109.6 \\
\hline $\mathrm{H}(67 \mathrm{~A})-\mathrm{C}(67)-\mathrm{H}(67 \mathrm{~B})$ & 109.5 & $\mathrm{H}(56 \mathrm{C})-\mathrm{C}\left(56^{\prime}\right)-\mathrm{H}(56 \mathrm{D})$ & 108.1 \\
\hline $\mathrm{C}(66)-\mathrm{C}(67)-\mathrm{H}(67 \mathrm{C})$ & 109.5 & $\mathrm{C}\left(66^{\prime}\right)-\mathrm{P}\left(4^{\prime}\right)-\mathrm{C}\left(63^{\prime}\right)$ & $104.4(11)$ \\
\hline $\mathrm{H}(67 \mathrm{~A})-\mathrm{C}(67)-\mathrm{H}(67 \mathrm{C})$ & 109.5 & $\mathrm{C}\left(66^{\prime}\right)-\mathrm{P}\left(4^{\prime}\right)-\mathrm{C}\left(56^{\prime}\right)$ & $123.1(13)$ \\
\hline $\mathrm{H}(67 \mathrm{~B})-\mathrm{C}(67)-\mathrm{H}(67 \mathrm{C})$ & 109.5 & $\mathrm{C}\left(63^{\prime}\right)-\mathrm{P}\left(4^{\prime}\right)-\mathrm{C}\left(56^{\prime}\right)$ & $90.2(8)$ \\
\hline $\mathrm{C}(66)-\mathrm{C}(68)-\mathrm{H}(68 \mathrm{~A})$ & 109.5 & $\mathrm{C}\left(66^{\prime}\right)-\mathrm{P}\left(4^{\prime}\right)-\mathrm{Pd}(2)$ & $113.6(6)$ \\
\hline $\mathrm{C}(66)-\mathrm{C}(68)-\mathrm{H}(68 \mathrm{~B})$ & 109.5 & $\mathrm{C}\left(63^{\prime}\right)-\mathrm{P}\left(4^{\prime}\right)-\mathrm{Pd}(2)$ & $125.0(8)$ \\
\hline $\mathrm{H}(68 \mathrm{~A})-\mathrm{C}(68)-\mathrm{H}(68 \mathrm{~B})$ & 109.5 & $\mathrm{C}\left(56^{\prime}\right)-\mathrm{P}\left(4^{\prime}\right)-\mathrm{Pd}(2)$ & $100.0(7)$ \\
\hline $\mathrm{C}(66)-\mathrm{C}(68)-\mathrm{H}(68 \mathrm{C})$ & 109.5 & $\mathrm{C}\left(58^{\prime}\right)-\mathrm{C}\left(57^{\prime}\right)-\mathrm{C}\left(59^{\prime}\right)$ & $106.6(17)$ \\
\hline $\mathrm{H}(68 \mathrm{~A})-\mathrm{C}(68)-\mathrm{H}(68 \mathrm{C})$ & 109.5 & $\mathrm{C}\left(58^{\prime}\right)-\mathrm{C}\left(57^{\prime}\right)-\mathrm{P}\left(3^{\prime}\right)$ & $111.0(14)$ \\
\hline H(68B)-C(68)-H(68C) & 109.5 & $\mathrm{C}\left(59^{\prime}\right)-\mathrm{C}\left(57^{\prime}\right)-\mathrm{P}\left(3^{\prime}\right)$ & $120(2)$ \\
\hline $\mathrm{N}(2)-\mathrm{C}\left(35^{\prime}\right)-\mathrm{C}\left(36^{\prime}\right)$ & $141(2)$ & $\mathrm{C}\left(58^{\prime}\right)-\mathrm{C}\left(57^{\prime}\right)-\mathrm{H}\left(57^{\prime}\right)$ & 106.1 \\
\hline $\mathrm{N}(2)-\mathrm{C}\left(35^{\prime}\right)-\mathrm{Pd}(2)$ & $76.7(18)$ & $\mathrm{C}\left(59^{\prime}\right)-\mathrm{C}\left(57^{\prime}\right)-\mathrm{H}\left(57^{\prime}\right)$ & 106.1 \\
\hline $\mathrm{C}\left(36^{\prime}\right)-\mathrm{C}\left(35^{\prime}\right)-\mathrm{Pd}(2)$ & $142.5(16)$ & $\mathrm{P}\left(3^{\prime}\right)-\mathrm{C}\left(57^{\prime}\right)-\mathrm{H}\left(57^{\prime}\right)$ & 106.1 \\
\hline $\mathrm{C}\left(35^{\prime}\right)-\mathrm{C}\left(36^{\prime}\right)-\mathrm{H}(36 \mathrm{D})$ & 109.5 & $\mathrm{C}\left(57^{\prime}\right)-\mathrm{C}\left(58^{\prime}\right)-\mathrm{H}(58 \mathrm{D})$ & 109.5 \\
\hline
\end{tabular}




\begin{tabular}{|c|c|c|c|}
\hline $\mathrm{C}\left(57^{\prime}\right)-\mathrm{C}\left(58^{\prime}\right)-\mathrm{H}(58 \mathrm{E})$ & 109.5 & $\mathrm{C}\left(63^{\prime}\right)-\mathrm{C}\left(64^{\prime}\right)-\mathrm{H}(64 \mathrm{E})$ & 109.5 \\
\hline $\mathrm{H}(58 \mathrm{D})-\mathrm{C}\left(58^{\prime}\right)-\mathrm{H}(58 \mathrm{E})$ & 109.5 & $\mathrm{H}(64 \mathrm{D})-\mathrm{C}\left(64^{\prime}\right)-\mathrm{H}(64 \mathrm{E})$ & 109.5 \\
\hline $\mathrm{C}\left(57^{\prime}\right)-\mathrm{C}\left(58^{\prime}\right)-\mathrm{H}(58 \mathrm{~F})$ & 109.5 & $\mathrm{C}\left(63^{\prime}\right)-\mathrm{C}\left(64^{\prime}\right)-\mathrm{H}(64 \mathrm{~F})$ & 109.5 \\
\hline $\mathrm{H}(58 \mathrm{D})-\mathrm{C}\left(58^{\prime}\right)-\mathrm{H}(58 \mathrm{~F})$ & 109.5 & $\mathrm{H}(64 \mathrm{D})-\mathrm{C}\left(64^{\prime}\right)-\mathrm{H}(64 \mathrm{~F})$ & 109.5 \\
\hline $\mathrm{H}(58 \mathrm{E})-\mathrm{C}\left(58^{\prime}\right)-\mathrm{H}(58 \mathrm{~F})$ & 109.5 & $\mathrm{H}(64 \mathrm{E})-\mathrm{C}\left(64^{\prime}\right)-\mathrm{H}(64 \mathrm{~F})$ & 109.5 \\
\hline $\mathrm{C}\left(57^{\prime}\right)-\mathrm{C}\left(59^{\prime}\right)-\mathrm{H}(59 \mathrm{D})$ & 109.5 & $\mathrm{C}\left(63^{\prime}\right)-\mathrm{C}\left(65^{\prime}\right)-\mathrm{H}(65 \mathrm{D})$ & 109.5 \\
\hline $\mathrm{C}\left(57^{\prime}\right)-\mathrm{C}\left(59^{\prime}\right)-\mathrm{H}(59 \mathrm{E})$ & 109.5 & $\mathrm{C}\left(63^{\prime}\right)-\mathrm{C}\left(65^{\prime}\right)-\mathrm{H}(65 \mathrm{E})$ & 109.5 \\
\hline $\mathrm{H}(59 \mathrm{D})-\mathrm{C}\left(59^{\prime}\right)-\mathrm{H}(59 \mathrm{E})$ & 109.5 & $\mathrm{H}(65 \mathrm{D})-\mathrm{C}\left(65^{\prime}\right)-\mathrm{H}(65 \mathrm{E})$ & 109.5 \\
\hline $\mathrm{C}\left(57^{\prime}\right)-\mathrm{C}\left(59^{\prime}\right)-\mathrm{H}(59 \mathrm{~F})$ & 109.5 & $\mathrm{C}\left(63^{\prime}\right)-\mathrm{C}\left(65^{\prime}\right)-\mathrm{H}(65 \mathrm{~F})$ & 109.5 \\
\hline $\mathrm{H}(59 \mathrm{D})-\mathrm{C}\left(59^{\prime}\right)-\mathrm{H}(59 \mathrm{~F})$ & 109.5 & $\mathrm{H}(65 \mathrm{D})-\mathrm{C}\left(65^{\prime}\right)-\mathrm{H}(65 \mathrm{~F})$ & 109.5 \\
\hline $\mathrm{H}(59 \mathrm{E})-\mathrm{C}\left(59^{\prime}\right)-\mathrm{H}(59 \mathrm{~F})$ & 109.5 & $\mathrm{H}(65 \mathrm{E})-\mathrm{C}\left(65^{\prime}\right)-\mathrm{H}(65 \mathrm{~F})$ & 109.5 \\
\hline $\mathrm{C}\left(61^{\prime}\right)-\mathrm{C}\left(60^{\prime}\right)-\mathrm{C}\left(62^{\prime}\right)$ & 111.1(19) & $C\left(68^{\prime}\right)-C\left(66^{\prime}\right)-C\left(67^{\prime}\right)$ & $114(3)$ \\
\hline $\mathrm{C}\left(61^{\prime}\right)-\mathrm{C}\left(60^{\prime}\right)-\mathrm{P}\left(3^{\prime}\right)$ & $119.6(13)$ & $\mathrm{C}\left(68^{\prime}\right)-\mathrm{C}\left(66^{\prime}\right)-\mathrm{P}\left(4^{\prime}\right)$ & $114.7(14)$ \\
\hline $\mathrm{C}\left(62^{\prime}\right)-\mathrm{C}\left(60^{\prime}\right)-\mathrm{P}\left(3^{\prime}\right)$ & $112.8(19)$ & $\mathrm{C}\left(67^{\prime}\right)-\mathrm{C}\left(66^{\prime}\right)-\mathrm{P}\left(4^{\prime}\right)$ & $103(2)$ \\
\hline $\mathrm{C}\left(61^{\prime}\right)-\mathrm{C}\left(60^{\prime}\right)-\mathrm{H}\left(60^{\prime}\right)$ & 103.8 & $\mathrm{C}\left(68^{\prime}\right)-\mathrm{C}\left(66^{\prime}\right)-\mathrm{H}\left(66^{\prime}\right)$ & 108.5 \\
\hline $\mathrm{C}\left(62^{\prime}\right)-\mathrm{C}\left(60^{\prime}\right)-\mathrm{H}\left(60^{\prime}\right)$ & 103.8 & $\mathrm{C}\left(67^{\prime}\right)-\mathrm{C}\left(66^{\prime}\right)-\mathrm{H}\left(66^{\prime}\right)$ & 108.5 \\
\hline $\mathrm{P}\left(3^{\prime}\right)-\mathrm{C}\left(60^{\prime}\right)-\mathrm{H}\left(60^{\prime}\right)$ & 103.8 & $\mathrm{P}\left(4^{\prime}\right)-\mathrm{C}\left(66^{\prime}\right)-\mathrm{H}\left(66^{\prime}\right)$ & 108.5 \\
\hline$C\left(60^{\prime}\right)-C\left(61^{\prime}\right)-H(61 D)$ & 109.5 & $\mathrm{C}\left(66^{\prime}\right)-\mathrm{C}\left(67^{\prime}\right)-\mathrm{H}(67 \mathrm{D})$ & 109.5 \\
\hline $\mathrm{C}\left(60^{\prime}\right)-\mathrm{C}\left(61^{\prime}\right)-\mathrm{H}(61 \mathrm{E})$ & 109.5 & $\mathrm{C}\left(66^{\prime}\right)-\mathrm{C}\left(67^{\prime}\right)-\mathrm{H}(67 \mathrm{E})$ & 109.5 \\
\hline $\mathrm{H}(61 \mathrm{D})-\mathrm{C}\left(61^{\prime}\right)-\mathrm{H}(61 \mathrm{E})$ & 109.5 & $\mathrm{H}(67 \mathrm{D})-\mathrm{C}\left(67^{\prime}\right)-\mathrm{H}(67 \mathrm{E})$ & 109.5 \\
\hline $\mathrm{C}\left(60^{\prime}\right)-\mathrm{C}\left(61^{\prime}\right)-\mathrm{H}(61 \mathrm{~F})$ & 109.5 & $\mathrm{C}\left(66^{\prime}\right)-\mathrm{C}\left(67^{\prime}\right)-\mathrm{H}(67 \mathrm{~F})$ & 109.5 \\
\hline $\mathrm{H}(61 \mathrm{D})-\mathrm{C}\left(61^{\prime}\right)-\mathrm{H}(61 \mathrm{~F})$ & 109.5 & $\mathrm{H}(67 \mathrm{D})-\mathrm{C}\left(67^{\prime}\right)-\mathrm{H}(67 \mathrm{~F})$ & 109.5 \\
\hline $\mathrm{H}(61 \mathrm{E})-\mathrm{C}\left(61^{\prime}\right)-\mathrm{H}(61 \mathrm{~F})$ & 109.5 & $\mathrm{H}(67 \mathrm{E})-\mathrm{C}\left(67^{\prime}\right)-\mathrm{H}(67 \mathrm{~F})$ & 109.5 \\
\hline$C\left(60^{\prime}\right)-C\left(62^{\prime}\right)-H(62 D)$ & 109.5 & $\mathrm{C}\left(66^{\prime}\right)-\mathrm{C}\left(68^{\prime}\right)-\mathrm{H}(68 \mathrm{D})$ & 109.5 \\
\hline $\mathrm{C}\left(60^{\prime}\right)-\mathrm{C}\left(62^{\prime}\right)-\mathrm{H}(62 \mathrm{E})$ & 109.5 & $\mathrm{C}\left(66^{\prime}\right)-\mathrm{C}\left(68^{\prime}\right)-\mathrm{H}(68 \mathrm{E})$ & 109.5 \\
\hline $\mathrm{H}(62 \mathrm{D})-\mathrm{C}\left(62^{\prime}\right)-\mathrm{H}(62 \mathrm{E})$ & 109.5 & $\mathrm{H}(68 \mathrm{D})-\mathrm{C}\left(68^{\prime}\right)-\mathrm{H}(68 \mathrm{E})$ & 109.5 \\
\hline $\mathrm{C}\left(60^{\prime}\right)-\mathrm{C}\left(62^{\prime}\right)-\mathrm{H}(62 \mathrm{~F})$ & 109.5 & $\mathrm{C}\left(66^{\prime}\right)-\mathrm{C}\left(68^{\prime}\right)-\mathrm{H}(68 \mathrm{~F})$ & 109.5 \\
\hline $\mathrm{H}(62 \mathrm{D})-\mathrm{C}\left(62^{\prime}\right)-\mathrm{H}(62 \mathrm{~F})$ & 109.5 & $\mathrm{H}(68 \mathrm{D})-\mathrm{C}\left(68^{\prime}\right)-\mathrm{H}(68 \mathrm{~F})$ & 109.5 \\
\hline $\mathrm{H}(62 \mathrm{E})-\mathrm{C}\left(62^{\prime}\right)-\mathrm{H}(62 \mathrm{~F})$ & 109.5 & $\mathrm{H}(68 \mathrm{E})-\mathrm{C}\left(68^{\prime}\right)-\mathrm{H}(68 \mathrm{~F})$ & 109.5 \\
\hline $\mathrm{C}\left(65^{\prime}\right)-\mathrm{C}\left(63^{\prime}\right)-\mathrm{C}\left(64^{\prime}\right)$ & $105(2)$ & $\mathrm{C}\left(35^{\prime}\right)-\mathrm{N}(2)-\mathrm{B}(2)$ & $141.8(17)$ \\
\hline $\mathrm{C}\left(65^{\prime}\right)-\mathrm{C}\left(63^{\prime}\right)-\mathrm{P}\left(4^{\prime}\right)$ & $109.8(13)$ & $\mathrm{C}(35)-\mathrm{N}(2)-\mathrm{B}(2)$ & $145.4(10)$ \\
\hline $\mathrm{C}\left(64^{\prime}\right)-\mathrm{C}\left(63^{\prime}\right)-\mathrm{P}\left(4^{\prime}\right)$ & $114.0(13)$ & $\mathrm{C}\left(35^{\prime}\right)-\mathrm{N}(2)-\mathrm{Pd}(2)$ & $70.8(17)$ \\
\hline $\mathrm{C}\left(65^{\prime}\right)-\mathrm{C}\left(63^{\prime}\right)-\mathrm{H}\left(63^{\prime}\right)$ & 109.4 & $\mathrm{C}(35)-\mathrm{N}(2)-\mathrm{Pd}(2)$ & $66.3(10)$ \\
\hline $\mathrm{C}\left(64^{\prime}\right)-\mathrm{C}\left(63^{\prime}\right)-\mathrm{H}\left(63^{\prime}\right)$ & 109.4 & $\mathrm{~B}(2)-\mathrm{N}(2)-\mathrm{Pd}(2)$ & $147.2(3)$ \\
\hline $\mathrm{P}\left(4^{\prime}\right)-\mathrm{C}\left(63^{\prime}\right)-\mathrm{H}\left(63^{\prime}\right)$ & 109.4 & $\mathrm{~N}(2)-\mathrm{B}(2)-\mathrm{C}(49)$ & $106.1(4)$ \\
\hline $\mathrm{C}\left(63^{\prime}\right)-\mathrm{C}\left(64^{\prime}\right)-\mathrm{H}(64 \mathrm{D})$ & 109.5 & $\mathrm{~N}(2)-\mathrm{B}(2)-\mathrm{C}(43)$ & $107.0(4)$ \\
\hline
\end{tabular}




\begin{tabular}{|c|c|c|c|}
\hline $\mathrm{C}(49)-\mathrm{B}(2)-\mathrm{C}(43)$ & $112.4(4)$ & $\mathrm{C}(46)-\mathrm{C}(45)-\mathrm{H}(45)$ & 119.8 \\
\hline $\mathrm{N}(2)-\mathrm{B}(2)-\mathrm{C}(37)$ & $104.2(4)$ & $\mathrm{C}(44)-\mathrm{C}(45)-\mathrm{H}(45)$ & 119.8 \\
\hline C(49)-B(2)-C(37) & $113.4(5)$ & $C(45)-C(46)-C(47)$ & $119.7(6)$ \\
\hline $\mathrm{C}(43)-\mathrm{B}(2)-\mathrm{C}(37)$ & $112.9(4)$ & $\mathrm{C}(45)-\mathrm{C}(46)-\mathrm{H}(46)$ & 120.2 \\
\hline $\mathrm{C}(42)-\mathrm{C}(37)-\mathrm{C}(38)$ & $115.8(4)$ & $\mathrm{C}(47)-\mathrm{C}(46)-\mathrm{H}(46)$ & 120.2 \\
\hline $\mathrm{C}(42)-\mathrm{C}(37)-\mathrm{B}(2)$ & $121.8(5)$ & $\mathrm{C}(46)-\mathrm{C}(47)-\mathrm{C}(48)$ & $119.0(6)$ \\
\hline $\mathrm{C}(38)-\mathrm{C}(37)-\mathrm{B}(2)$ & $122.2(5)$ & $\mathrm{C}(46)-\mathrm{C}(47)-\mathrm{H}(47)$ & 120.5 \\
\hline $\mathrm{C}(39)-\mathrm{C}(38)-\mathrm{C}(37)$ & $121.9(5)$ & $\mathrm{C}(48)-\mathrm{C}(47)-\mathrm{H}(47)$ & 120.5 \\
\hline $\mathrm{C}(39)-\mathrm{C}(38)-\mathrm{H}(38)$ & 119.0 & $\mathrm{C}(47)-\mathrm{C}(48)-\mathrm{C}(43)$ & $123.1(6)$ \\
\hline $\mathrm{C}(37)-\mathrm{C}(38)-\mathrm{H}(38)$ & 119.0 & $\mathrm{C}(47)-\mathrm{C}(48)-\mathrm{H}(48)$ & 118.5 \\
\hline $\mathrm{C}(40)-\mathrm{C}(39)-\mathrm{C}(38)$ & $120.6(5)$ & $\mathrm{C}(43)-\mathrm{C}(48)-\mathrm{H}(48)$ & 118.5 \\
\hline $\mathrm{C}(40)-\mathrm{C}(39)-\mathrm{H}(39)$ & 119.7 & $\mathrm{C}(54)-\mathrm{C}(49)-\mathrm{C}(50)$ & $115.9(5)$ \\
\hline $\mathrm{C}(38)-\mathrm{C}(39)-\mathrm{H}(39)$ & 119.7 & $\mathrm{C}(54)-\mathrm{C}(49)-\mathrm{B}(2)$ & $122.8(5)$ \\
\hline$C(39)-C(40)-C(41)$ & $119.7(5)$ & $\mathrm{C}(50)-\mathrm{C}(49)-\mathrm{B}(2)$ & $121.3(5)$ \\
\hline $\mathrm{C}(39)-\mathrm{C}(40)-\mathrm{H}(40)$ & 120.2 & $\mathrm{C}(51)-\mathrm{C}(50)-\mathrm{C}(49)$ & $122.6(5)$ \\
\hline $\mathrm{C}(41)-\mathrm{C}(40)-\mathrm{H}(40)$ & 120.2 & $\mathrm{C}(51)-\mathrm{C}(50)-\mathrm{H}(50)$ & 118.7 \\
\hline$C(40)-C(41)-C(42)$ & $119.7(6)$ & $\mathrm{C}(49)-\mathrm{C}(50)-\mathrm{H}(50)$ & 118.7 \\
\hline $\mathrm{C}(40)-\mathrm{C}(41)-\mathrm{H}(41)$ & 120.1 & $\mathrm{C}(50)-\mathrm{C}(51)-\mathrm{C}(52)$ & $120.1(6)$ \\
\hline $\mathrm{C}(42)-\mathrm{C}(41)-\mathrm{H}(41)$ & 120.1 & $\mathrm{C}(50)-\mathrm{C}(51)-\mathrm{H}(51)$ & 120.0 \\
\hline$C(37)-C(42)-C(41)$ & $122.3(5)$ & $\mathrm{C}(52)-\mathrm{C}(51)-\mathrm{H}(51)$ & 120.0 \\
\hline $\mathrm{C}(37)-\mathrm{C}(42)-\mathrm{H}(42)$ & 118.9 & $\mathrm{C}(53)-\mathrm{C}(52)-\mathrm{C}(51)$ & $118.4(6)$ \\
\hline $\mathrm{C}(41)-\mathrm{C}(42)-\mathrm{H}(42)$ & 118.9 & $\mathrm{C}(53)-\mathrm{C}(52)-\mathrm{H}(52)$ & 120.8 \\
\hline$C(44)-C(43)-C(48)$ & $115.5(5)$ & $\mathrm{C}(51)-\mathrm{C}(52)-\mathrm{H}(52)$ & 120.8 \\
\hline $\mathrm{C}(44)-\mathrm{C}(43)-\mathrm{B}(2)$ & $123.7(5)$ & $\mathrm{C}(54)-\mathrm{C}(53)-\mathrm{C}(52)$ & $120.6(6)$ \\
\hline $\mathrm{C}(48)-\mathrm{C}(43)-\mathrm{B}(2)$ & $120.6(5)$ & $\mathrm{C}(54)-\mathrm{C}(53)-\mathrm{H}(53)$ & 119.7 \\
\hline$C(43)-C(44)-C(45)$ & $122.2(6)$ & $\mathrm{C}(52)-\mathrm{C}(53)-\mathrm{H}(53)$ & 119.7 \\
\hline $\mathrm{C}(43)-\mathrm{C}(44)-\mathrm{H}(44)$ & 118.9 & $\mathrm{C}(53)-\mathrm{C}(54)-\mathrm{C}(49)$ & $122.4(6)$ \\
\hline $\mathrm{C}(45)-\mathrm{C}(44)-\mathrm{H}(44)$ & 118.9 & $\mathrm{C}(53)-\mathrm{C}(54)-\mathrm{H}(54)$ & 118.8 \\
\hline$C(46)-C(45)-C(44)$ & $120.5(6)$ & $\mathrm{C}(49)-\mathrm{C}(54)-\mathrm{H}(54)$ & 118.8 \\
\hline
\end{tabular}


Table S 16. Anisotropic displacement parameters $\left(\AA^{2} \times 10^{3}\right)$ for jonlm 37 (2a'). The anisotropic displacement factor exponent takes the form: $-2 \pi^{2}\left[\mathrm{~h}^{2} \mathrm{a}^{* 2} \mathrm{U}_{11}+\ldots+2 \mathrm{~h} \mathrm{k} \mathrm{a}^{*} \mathrm{~b}^{*} \mathrm{U}_{12}\right]$

\begin{tabular}{|c|c|c|c|c|c|c|}
\hline & $\mathrm{U}_{11}$ & $\mathrm{U}_{22}$ & $\mathrm{U}_{33}$ & $\mathrm{U}_{23}$ & $\mathrm{U}_{13}$ & $\mathrm{U}_{12}$ \\
\hline Pd1 & $24(1)$ & $12(1)$ & $21(1)$ & $-2(1)$ & $-1(1)$ & $0(1)$ \\
\hline P1 & $28(1)$ & $16(1)$ & $32(1)$ & $3(1)$ & $0(1)$ & $-1(1)$ \\
\hline P2 & $30(1)$ & $18(1)$ & $31(1)$ & $-6(1)$ & $10(1)$ & $-2(1)$ \\
\hline N1 & $29(2)$ & $14(2)$ & $22(2)$ & $1(2)$ & $-1(2)$ & $2(2)$ \\
\hline B1 & $28(3)$ & $16(2)$ & $18(3)$ & $-4(2)$ & $-4(2)$ & $0(2)$ \\
\hline $\mathrm{C} 1$ & $24(3)$ & $12(2)$ & $32(3)$ & $-2(2)$ & $-4(2)$ & $4(2)$ \\
\hline $\mathrm{C} 2$ & $44(3)$ & $24(3)$ & $38(4)$ & $-17(3)$ & $-3(3)$ & $1(2)$ \\
\hline $\mathrm{C} 3$ & $20(2)$ & $15(2)$ & $25(3)$ & $-2(2)$ & $-5(2)$ & $0(2)$ \\
\hline $\mathrm{C} 4$ & $37(3)$ & $16(2)$ & $37(4)$ & $0(2)$ & $4(2)$ & $3(2)$ \\
\hline $\mathrm{C} 5$ & $37(3)$ & $22(3)$ & $50(4)$ & $-9(3)$ & $3(3)$ & $5(2)$ \\
\hline C6 & $39(3)$ & $35(3)$ & $34(4)$ & $-9(3)$ & $2(3)$ & $6(3)$ \\
\hline C7 & $45(4)$ & $36(3)$ & $26(3)$ & $-5(2)$ & $1(3)$ & $8(3)$ \\
\hline $\mathrm{C} 8$ & $24(2)$ & $27(3)$ & $28(3)$ & $-2(2)$ & $0(2)$ & $7(2)$ \\
\hline C9 & $22(2)$ & $14(2)$ & $18(2)$ & $-4(2)$ & $3(2)$ & $-3(2)$ \\
\hline $\mathrm{C} 10$ & $42(3)$ & $18(2)$ & $20(3)$ & $-8(2)$ & $-2(2)$ & $1(2)$ \\
\hline $\mathrm{C} 11$ & $45(3)$ & $18(2)$ & $24(3)$ & $2(2)$ & $9(2)$ & $9(2)$ \\
\hline $\mathrm{C} 12$ & $50(3)$ & $14(2)$ & $35(3)$ & $-1(2)$ & $13(3)$ & $-2(2)$ \\
\hline $\mathrm{C} 13$ & $35(3)$ & $23(3)$ & $64(5)$ & $-14(3)$ & $-7(3)$ & $-6(2)$ \\
\hline C14 & $28(3)$ & $21(2)$ & $36(3)$ & $-2(2)$ & $-4(2)$ & $0(2)$ \\
\hline $\mathrm{C} 15$ & $32(3)$ & $10(2)$ & $21(3)$ & $-1(2)$ & $-6(2)$ & $0(2)$ \\
\hline $\mathrm{C} 16$ & $32(3)$ & $21(2)$ & $22(3)$ & $1(2)$ & $-6(2)$ & $-2(2)$ \\
\hline $\mathrm{C} 17$ & $38(3)$ & $30(3)$ & $24(3)$ & $3(2)$ & $-1(2)$ & $-7(2)$ \\
\hline C18 & $43(3)$ & $25(2)$ & $20(3)$ & $4(2)$ & $-7(2)$ & $0(2)$ \\
\hline C19 & $30(3)$ & $31(3)$ & $32(3)$ & $5(2)$ & $-3(2)$ & $0(2)$ \\
\hline $\mathrm{C} 20$ & $21(2)$ & $31(3)$ & $34(3)$ & $7(2)$ & $2(2)$ & $2(2)$ \\
\hline $\mathrm{C} 21$ & $33(3)$ & $24(2)$ & $34(3)$ & $9(2)$ & $8(3)$ & $2(2)$ \\
\hline $\mathrm{C} 22$ & $35(3)$ & $19(2)$ & $40(3)$ & $-1(2)$ & $8(3)$ & $5(2)$ \\
\hline $\mathrm{C} 23$ & $29(3)$ & $29(3)$ & $42(4)$ & $4(2)$ & $-6(3)$ & $-2(2)$ \\
\hline $\mathrm{C} 24$ & $49(4)$ & $38(3)$ & $34(4)$ & $5(3)$ & $-10(3)$ & $7(3)$ \\
\hline $\mathrm{C} 25$ & $69(5)$ & $39(4)$ & $50(5)$ & $-16(3)$ & $-26(4)$ & $3(4)$ \\
\hline C26 & $32(3)$ & $15(2)$ & $45(4)$ & $8(2)$ & $-6(3)$ & $-1(2)$ \\
\hline
\end{tabular}




\begin{tabular}{|c|c|c|c|c|c|c|}
\hline $\mathrm{C} 27$ & $46(4)$ & $27(3)$ & $56(5)$ & $16(3)$ & $-2(3)$ & $-2(3)$ \\
\hline $\mathrm{C} 28$ & $40(3)$ & $25(3)$ & $64(5)$ & $14(3)$ & $-6(3)$ & $-9(2)$ \\
\hline $\mathrm{C} 29$ & $51(4)$ & $20(2)$ & $44(4)$ & $2(2)$ & $21(3)$ & $-2(2)$ \\
\hline $\mathrm{C} 30$ & $68(5)$ & $23(3)$ & $50(4)$ & $10(3)$ & $18(4)$ & $1(3)$ \\
\hline $\mathrm{C} 31$ & $75(5)$ & $17(3)$ & $76(6)$ & $-8(3)$ & $40(4)$ & $-4(3)$ \\
\hline $\mathrm{C} 32$ & $44(4)$ & $31(3)$ & $35(3)$ & $-15(2)$ & $14(3)$ & $-2(3)$ \\
\hline $\mathrm{C} 33$ & $50(4)$ & $64(4)$ & $31(4)$ & $-15(3)$ & $10(3)$ & $-10(4)$ \\
\hline $\mathrm{C} 34$ & $38(4)$ & $42(3)$ & $48(4)$ & $-21(3)$ & $4(3)$ & $-4(3)$ \\
\hline $\mathrm{Pd} 2$ & $30(1)$ & $19(1)$ & $28(1)$ & $-3(1)$ & $0(1)$ & $9(1)$ \\
\hline $\mathrm{C} 35$ & $26(2)$ & $17(2)$ & $68(6)$ & $-10(5)$ & $-11(5)$ & $4(2)$ \\
\hline $\mathrm{C} 36$ & $26(2)$ & $17(2)$ & $68(6)$ & $-10(5)$ & $-11(5)$ & $4(2)$ \\
\hline P3 & $20(1)$ & $43(2)$ & $48(1)$ & $-3(1)$ & $-6(1)$ & $17(1)$ \\
\hline C55 & $31(4)$ & $41(6)$ & $42(5)$ & $-3(5)$ & $-6(3)$ & 19(4) \\
\hline C56 & $47(5)$ & $41(5)$ & $74(6)$ & $-12(5)$ & $-24(5)$ & $20(4)$ \\
\hline P4 & $47(2)$ & $27(1)$ & $45(2)$ & $12(1)$ & $20(1)$ & 19(1) \\
\hline C57 & $29(3)$ & $32(6)$ & $63(5)$ & $5(5)$ & $-17(4)$ & $-10(5)$ \\
\hline C58 & $42(6)$ & $53(8)$ & $42(5)$ & $17(7)$ & $1(5)$ & $-16(5)$ \\
\hline C59 & $40(8)$ & $30(7)$ & $85(11)$ & $9(6)$ & $-12(7)$ & $-8(5)$ \\
\hline C60 & $32(4)$ & $32(6)$ & $66(9)$ & $6(5)$ & $-8(5)$ & $-5(5)$ \\
\hline C61 & $62(7)$ & $46(7)$ & $87(11)$ & $-40(7)$ & $25(7)$ & $-21(7)$ \\
\hline C62 & $28(4)$ & $41(10)$ & $68(13)$ & $0(7)$ & $-10(6)$ & $4(5)$ \\
\hline C63 & $64(9)$ & $23(5)$ & $105(13)$ & $-5(6)$ & $28(7)$ & $5(5)$ \\
\hline C64 & $105(11)$ & $63(9)$ & $90(13)$ & $-43(8)$ & 49(9) & $-32(8)$ \\
\hline C65 & $116(11)$ & $29(5)$ & $159(16)$ & $4(8)$ & $75(12)$ & $26(6)$ \\
\hline C66 & $66(8)$ & $98(10)$ & $81(10)$ & $50(8)$ & $20(7)$ & $26(7)$ \\
\hline C67 & $74(10)$ & 189(19) & $52(9)$ & $44(14)$ & $-8(7)$ & $22(15)$ \\
\hline C68 & $100(9)$ & $61(7)$ & $109(11)$ & $49(7)$ & $48(8)$ & $47(7)$ \\
\hline $\mathrm{C} 35^{\prime}$ & $26(2)$ & $17(2)$ & $68(6)$ & $-10(5)$ & $-11(5)$ & $4(2)$ \\
\hline $\mathrm{C} 36^{\prime}$ & $26(2)$ & $17(2)$ & $68(6)$ & $-10(5)$ & $-11(5)$ & $4(2)$ \\
\hline P3' & $20(1)$ & $43(2)$ & $48(1)$ & $-3(1)$ & $-6(1)$ & $17(1)$ \\
\hline C55' & $31(4)$ & $41(6)$ & $42(5)$ & $-3(5)$ & $-6(3)$ & 19(4) \\
\hline C56' & $47(5)$ & $41(5)$ & $74(6)$ & $-12(5)$ & $-24(5)$ & $20(4)$ \\
\hline P4' & $47(2)$ & $27(1)$ & $45(2)$ & $12(1)$ & $20(1)$ & 19(1) \\
\hline C57' & $29(3)$ & $32(6)$ & $63(5)$ & $5(5)$ & $-17(4)$ & $-10(5)$ \\
\hline C58' & $42(6)$ & $53(8)$ & $42(5)$ & $17(7)$ & $1(5)$ & $-16(5)$ \\
\hline C59' & $40(8)$ & $30(7)$ & $85(11)$ & $9(6)$ & $-12(7)$ & $-8(5)$ \\
\hline
\end{tabular}




$\begin{array}{lcccccc}\text { C60' } & 32(4) & 32(6) & 66(9) & 6(5) & -8(5) & -5(5) \\ \text { C61' } & 62(7) & 46(7) & 87(11) & -40(7) & 25(7) & -21(7) \\ \text { C62' } & 28(4) & 41(10) & 68(13) & 0(7) & -10(6) & 4(5) \\ \text { C63' } & 64(9) & 23(5) & 105(13) & -5(6) & 28(7) & 5(5) \\ \text { C64' } & 105(11) & 63(9) & 90(13) & -43(8) & 49(9) & -32(8) \\ \text { C65' } & 116(11) & 29(5) & 159(16) & 4(8) & 75(12) & 26(6) \\ \text { C66' } & 66(8) & 98(10) & 81(10) & 50(8) & 20(7) & 26(7) \\ \text { C67' } & 74(10) & 189(19) & 52(9) & 44(14) & -8(7) & 22(15) \\ \text { C68' } & 100(9) & 61(7) & 109(11) & 49(7) & 48(8) & 47(7) \\ \text { N2 } & 30(2) & 22(2) & 26(3) & 0(2) & 7(2) & 6(2) \\ \text { B2 } & 31(3) & 16(2) & 19(3) & -5(2) & -1(2) & -2(2) \\ \text { C37 } & 23(2) & 17(2) & 27(3) & 0(2) & 1(2) & -1(2) \\ \text { C38 } & 36(3) & 20(2) & 28(3) & 5(2) & 4(2) & 2(2) \\ \text { C39 } & 42(3) & 19(2) & 36(4) & 7(2) & -5(3) & -2(2) \\ \text { C40 } & 29(3) & 18(2) & 46(4) & 2(2) & -4(3) & 3(2) \\ \text { C41 } & 36(3) & 24(3) & 31(3) & -4(2) & -1(2) & 7(2) \\ \text { C42 } & 35(3) & 20(2) & 22(3) & -2(2) & -5(2) & 6(2) \\ \text { C43 } & 35(3) & 15(2) & 19(3) & 2(2) & 2(2) & -1(2) \\ \text { C44 } & 43(3) & 24(2) & 27(3) & -3(2) & 9(3) & 2(2) \\ \text { C45 } & 54(4) & 27(3) & 32(4) & -4(2) & 16(3) & 9(3) \\ \text { C46 } & 69(4) & 20(3) & 25(3) & -6(2) & 3(3) & 6(3) \\ \text { C47 } & 57(4) & 28(3) & 25(3) & -2(2) & -5(3) & 1(3) \\ \text { C48 } & 44(3) & 21(2) & 28(3) & -1(2) & 1(3) & 0(2) \\ \text { C49 } & 29(3) & 20(2) & 27(3) & 1(2) & 3(2) & 4(2) \\ \text { C50 } & 22(2) & 24(3) & 31(3) & 0(2) & 0(2) & 10(2) \\ \text { C51 } & 39(3) & 38(3) & 20(3) & -4(2) & -2(2) & 7(3) \\ \text { C52 } & 45(4) & 31(3) & 23(3) & 6(2) & 2(3) & 6(3) \\ \text { C53 } & 52(4) & 24(3) & 32(3) & 6(2) & -5(3) & -1(3) \\ & 40(3) & 21(2) & 31(3) & -3(2) & -3(3) & -4(2)\end{array}$


Table S17. Hydrogen coordinates $\left(\times 10^{4}\right)$ and isotropic displacement parameters $\left(\AA^{2} \times 10^{3}\right)$ for jonlm37 (2a').

\begin{tabular}{|c|c|c|c|c|}
\hline & $\mathrm{x}$ & $y$ & z & $\mathrm{U}(\mathrm{eq})$ \\
\hline $\mathrm{H} 2 \mathrm{~A}$ & -542 & 4420 & 2346 & 53 \\
\hline $\mathrm{H} 2 \mathrm{~B}$ & -39 & 4771 & 1827 & 53 \\
\hline $\mathrm{H} 2 \mathrm{C}$ & 161 & 4338 & 2648 & 53 \\
\hline H4 & -1653 & 4383 & 1315 & 35 \\
\hline H5 & -2171 & 4946 & 2312 & 43 \\
\hline H6 & -2382 & 4312 & 3486 & 43 \\
\hline $\mathrm{H} 7$ & -2071 & 3121 & 3641 & 43 \\
\hline $\mathrm{H} 8$ & -1563 & 2562 & 2643 & 32 \\
\hline H10 & -606 & 1969 & 2177 & 33 \\
\hline H11 & -656 & 730 & 2186 & 35 \\
\hline H12 & -1274 & 121 & 1189 & 39 \\
\hline $\mathrm{H} 13$ & -1825 & 778 & 172 & 49 \\
\hline H14 & -1804 & 2023 & 190 & 34 \\
\hline H16 & -464 & 3524 & -84 & 31 \\
\hline H17 & -730 & 3902 & -1398 & 37 \\
\hline H18 & -1753 & 3952 & -1917 & 36 \\
\hline H19 & -2503 & 3646 & -1054 & 38 \\
\hline $\mathrm{H} 20$ & -2234 & 3294 & 275 & 34 \\
\hline $\mathrm{H} 21 \mathrm{~A}$ & 1667 & 3006 & -291 & 36 \\
\hline $\mathrm{H} 21 \mathrm{~B}$ & 2183 & 2826 & 423 & 36 \\
\hline $\mathrm{H} 22 \mathrm{~A}$ & 1713 & 1799 & 787 & 38 \\
\hline $\mathrm{H} 22 \mathrm{~B}$ & 1606 & 1787 & -189 & 38 \\
\hline $\mathrm{H} 23$ & 2309 & 3695 & 1728 & 41 \\
\hline $\mathrm{H} 24 \mathrm{~A}$ & 2308 & 2832 & 2780 & 62 \\
\hline $\mathrm{H} 24 \mathrm{~B}$ & 2186 & 2450 & 1909 & 62 \\
\hline $\mathrm{H} 24 \mathrm{C}$ & 1635 & 2593 & 2459 & 62 \\
\hline $\mathrm{H} 25 \mathrm{~A}$ & 1944 & 4064 & 2983 & 82 \\
\hline $\mathrm{H} 25 \mathrm{~B}$ & 1267 & 3876 & 2625 & 82 \\
\hline $\mathrm{H} 25 \mathrm{C}$ & 1629 & 4512 & 2230 & 82 \\
\hline H26 & 1305 & 4686 & 877 & 38 \\
\hline
\end{tabular}




\begin{tabular}{|c|c|c|c|c|}
\hline $\mathrm{H} 27 \mathrm{~A}$ & 908 & 4877 & -456 & 65 \\
\hline $\mathrm{H} 27 \mathrm{~B}$ & 522 & 4262 & -60 & 65 \\
\hline $\mathrm{H} 27 \mathrm{C}$ & 1010 & 4054 & -685 & 65 \\
\hline $\mathrm{H} 28 \mathrm{~A}$ & 2005 & 5002 & -29 & 65 \\
\hline $\mathrm{H} 28 \mathrm{~B}$ & 2141 & 4175 & -199 & 65 \\
\hline $\mathrm{H} 28 \mathrm{C}$ & 2319 & 4510 & 686 & 65 \\
\hline $\mathrm{H} 29$ & 124 & 1069 & 930 & 45 \\
\hline $\mathrm{H} 30 \mathrm{~A}$ & 700 & 782 & 2119 & 69 \\
\hline H30B & 609 & 1633 & 2063 & 69 \\
\hline $\mathrm{H} 30 \mathrm{C}$ & 1242 & 1283 & 1870 & 69 \\
\hline $\mathrm{H} 31 \mathrm{~A}$ & 747 & 56 & 859 & 81 \\
\hline H31B & 1272 & 540 & 531 & 81 \\
\hline $\mathrm{H} 31 \mathrm{C}$ & 644 & 434 & -17 & 81 \\
\hline H32 & 574 & 1370 & -876 & 43 \\
\hline $\mathrm{H} 33 \mathrm{~A}$ & 335 & 2313 & -1801 & 72 \\
\hline H33B & 932 & 2510 & -1222 & 72 \\
\hline $\mathrm{H} 33 \mathrm{C}$ & 294 & 2862 & -1058 & 72 \\
\hline $\mathrm{H} 34 \mathrm{~A}$ & -480 & 1585 & -1280 & 64 \\
\hline H34B & -523 & 2062 & -476 & 64 \\
\hline $\mathrm{H} 34 \mathrm{C}$ & -387 & 1220 & -396 & 64 \\
\hline $\mathrm{H} 36 \mathrm{~A}$ & 4155 & 4208 & 5340 & 57 \\
\hline H36B & 4838 & 4422 & 5196 & 57 \\
\hline $\mathrm{H} 36 \mathrm{C}$ & 4633 & 4371 & 6105 & 57 \\
\hline $\mathrm{H} 55 \mathrm{~A}$ & 7281 & 2711 & 6398 & 46 \\
\hline H55B & 6878 & 2662 & 7158 & 46 \\
\hline H56A & 6898 & 1553 & 6860 & 67 \\
\hline H56B & 6987 & 1698 & 5920 & 67 \\
\hline H57 & 7077 & 3813 & 5394 & 51 \\
\hline $\mathrm{H} 58 \mathrm{~A}$ & 6353 & 2847 & 4365 & 68 \\
\hline H58B & 7019 & 2674 & 4778 & 68 \\
\hline $\mathrm{H} 58 \mathrm{C}$ & 6926 & 3274 & 4082 & 68 \\
\hline $\mathrm{H} 59 \mathrm{~A}$ & 6367 & 4407 & 4379 & 79 \\
\hline H59B & 6299 & 4622 & 5309 & 79 \\
\hline $\mathrm{H} 59 \mathrm{C}$ & 5835 & 4064 & 4853 & 79 \\
\hline H60 & 6273 & 4438 & 6267 & 53 \\
\hline H61A & 6172 & 3679 & 7737 & 96 \\
\hline
\end{tabular}




\begin{tabular}{|c|c|c|c|c|}
\hline H61B & 5608 & 3839 & 7086 & 96 \\
\hline H61C & 5967 & 4489 & 7544 & 96 \\
\hline H62A & 7080 & 4658 & 7286 & 70 \\
\hline H62B & 7302 & 4352 & 6452 & 70 \\
\hline H62C & 7274 & 3834 & 7225 & 70 \\
\hline H63 & 5539 & 663 & 5302 & 75 \\
\hline H64A & 6395 & 1474 & 4512 & 125 \\
\hline H64B & 5675 & 1472 & 4308 & 125 \\
\hline H64C & 6064 & 778 & 4115 & 125 \\
\hline H65A & 6354 & -85 & 5142 & 147 \\
\hline H65B & 6315 & 85 & 6091 & 147 \\
\hline H65C & 6811 & 480 & 5598 & 147 \\
\hline H66 & 6098 & 849 & 7233 & 97 \\
\hline H67A & 5311 & 2013 & 7543 & 158 \\
\hline H67B & 6027 & 2008 & 7791 & 158 \\
\hline $\mathrm{H} 67 \mathrm{C}$ & 5595 & 1460 & 8218 & 158 \\
\hline H68A & 5069 & 488 & 7389 & 132 \\
\hline H68B & 5300 & 273 & 6525 & 132 \\
\hline H68C & 4862 & 949 & 6591 & 132 \\
\hline H36D & 4968 & 4388 & 5127 & 57 \\
\hline H36E & 4408 & 4110 & 4528 & 57 \\
\hline $\mathrm{H} 36 \mathrm{~F}$ & 4306 & 4337 & 5446 & 57 \\
\hline $\mathrm{H} 55 \mathrm{C}$ & 7233 & 2438 & 6271 & 46 \\
\hline H55D & 6857 & 2386 & 7055 & 46 \\
\hline H56C & 6835 & 1305 & 6516 & 67 \\
\hline H56D & 6671 & 1607 & 5610 & 67 \\
\hline H57' & 7093 & 3502 & 5243 & 51 \\
\hline H58D & 6788 & 2343 & 4790 & 68 \\
\hline H58E & 6202 & 2691 & 4302 & 68 \\
\hline $\mathrm{H} 58 \mathrm{~F}$ & 6866 & 2889 & 4054 & 68 \\
\hline H59D & 6403 & 4464 & 4997 & 79 \\
\hline H59E & 6638 & 4145 & 4176 & 79 \\
\hline $\mathrm{H} 59 \mathrm{~F}$ & 5975 & 3946 & 4424 & 79 \\
\hline H60' & 6169 & 4198 & 6726 & 53 \\
\hline H61D & 5666 & 3399 & 7537 & 96 \\
\hline H61E & 6307 & 3199 & 8010 & 96 \\
\hline
\end{tabular}




\begin{tabular}{|c|c|c|c|c|}
\hline $\mathrm{H} 61 \mathrm{~F}$ & 6052 & 4001 & 8045 & 96 \\
\hline H62D & 7225 & 4157 & 6680 & 70 \\
\hline H62E & 7013 & 4468 & 7517 & 70 \\
\hline H62F & 7268 & 3666 & 7482 & 70 \\
\hline H63' & 6005 & 380 & 6075 & 75 \\
\hline H64D & 6299 & 984 & 4889 & 125 \\
\hline H64E & 5862 & 322 & 4638 & 125 \\
\hline H64F & 5600 & 1123 & 4596 & 125 \\
\hline H65D & 4947 & 383 & 6257 & 147 \\
\hline H65E & 4814 & 774 & 5393 & 147 \\
\hline $\mathrm{H} 65 \mathrm{~F}$ & 5076 & -27 & 5434 & 147 \\
\hline H66' & 5015 & 1298 & 7234 & 97 \\
\hline H67D & 5392 & 2461 & 7507 & 158 \\
\hline H67E & 5415 & 2012 & 8339 & 158 \\
\hline H67F & 6022 & 2138 & 7895 & 158 \\
\hline H68D & 5648 & 287 & 7394 & 132 \\
\hline H68E & 6183 & 767 & 7824 & 132 \\
\hline H68F & 5576 & 641 & 8268 & 132 \\
\hline H38 & 3291 & 3293 & 4068 & 34 \\
\hline H39 & 2722 & 4346 & 4055 & 39 \\
\hline $\mathrm{H} 40$ & 2400 & 4803 & 5249 & 38 \\
\hline H41 & 2639 & 4204 & 6479 & 37 \\
\hline $\mathrm{H} 42$ & 3209 & 3144 & 6501 & 32 \\
\hline H44 & 4704 & 1885 & 4381 & 37 \\
\hline $\mathrm{H} 45$ & 4649 & 1190 & 3204 & 44 \\
\hline H46 & 3717 & 874 & 2549 & 46 \\
\hline H47 & 2824 & 1253 & 3085 & 45 \\
\hline $\mathrm{H} 48$ & 2884 & 1934 & 4276 & 37 \\
\hline H50 & 4154 & 2590 & 6981 & 31 \\
\hline H51 & 4067 & 1873 & 8110 & 39 \\
\hline H52 & 3622 & 726 & 7956 & 40 \\
\hline H53 & 3317 & 311 & 6635 & 43 \\
\hline H54 & 3382 & 1050 & 5513 & 37 \\
\hline
\end{tabular}


Table S18. Torsion angles $\left[{ }^{\circ}\right]$ for jonlm37 (2a').

\begin{tabular}{|c|c|c|c|}
\hline C1-N1-B1-C15 & $84.7(9)$ & C12-C13-C14-C9 & $2.4(10)$ \\
\hline Pd1-N1-B1-C15 & $-64.7(6)$ & C10-C9-C14-C13 & $-1.8(9)$ \\
\hline C1-N1-B1-C3 & $-36.2(10)$ & B1-C9-C14-C13 & $178.4(6)$ \\
\hline Pd1-N1-B1-C3 & $174.4(4)$ & N1-B1-C15-C16 & $17.4(6)$ \\
\hline C1-N1-B1-C9 & $-157.3(8)$ & C3-B1-C15-C16 & $134.3(5)$ \\
\hline Pd1-N1-B1-C9 & $53.3(7)$ & C9-B1-C15-C16 & $-97.7(6)$ \\
\hline B1-N1-C1-C2 & $16.6(14)$ & N1-B1-C15-C20 & $-166.0(4)$ \\
\hline Pd1-N1-C1-C2 & $177.3(8)$ & C3-B1-C15-C20 & $-49.1(6)$ \\
\hline B1-N1-C1-Pd1 & $-160.7(8)$ & C9-B1-C15-C20 & $78.9(6)$ \\
\hline N1-B1-C3-C8 & $-92.0(5)$ & $\mathrm{C} 20-\mathrm{C} 15-\mathrm{C} 16-\mathrm{C} 17$ & $-1.0(7)$ \\
\hline $\mathrm{C} 15-\mathrm{B} 1-\mathrm{C} 3-\mathrm{C} 8$ & $151.9(5)$ & B1-C15-C16-C17 & $175.8(5)$ \\
\hline C9-B1-C3-C8 & $25.0(7)$ & C15-C16-C17-C18 & $-0.5(8)$ \\
\hline N1-B1-C3-C4 & $85.0(6)$ & C16-C17-C18-C19 & $1.2(8)$ \\
\hline C15-B1-C3-C4 & $-31.2(7)$ & C17-C18-C19-C20 & $-0.4(9)$ \\
\hline C9-B1-C3-C4 & $-158.1(5)$ & C18-C19-C20-C15 & $-1.2(9)$ \\
\hline $\mathrm{C} 8-\mathrm{C} 3-\mathrm{C} 4-\mathrm{C} 5$ & $0.3(8)$ & C16-C15-C20-C19 & $1.9(8)$ \\
\hline $\mathrm{B} 1-\mathrm{C} 3-\mathrm{C} 4-\mathrm{C} 5$ & $-176.9(5)$ & B1-C15-C20-C19 & $-175.0(5)$ \\
\hline C3-C4-C5-C6 & $0.1(9)$ & C23-P1-C21-C22 & $98.8(5)$ \\
\hline C4-C5-C6-C7 & $-0.1(9)$ & C26-P1-C21-C22 & $-151.5(4)$ \\
\hline $\mathrm{C} 5-\mathrm{C} 6-\mathrm{C} 7-\mathrm{C} 8$ & $-0.2(10)$ & Pd1-P1-C21-C22 & $-30.3(5)$ \\
\hline $\mathrm{C} 6-\mathrm{C} 7-\mathrm{C} 8-\mathrm{C} 3$ & $0.6(9)$ & $\mathrm{P} 1-\mathrm{C} 21-\mathrm{C} 22-\mathrm{P} 2$ & $40.0(6)$ \\
\hline $\mathrm{C} 4-\mathrm{C} 3-\mathrm{C} 8-\mathrm{C} 7$ & $-0.6(8)$ & $\mathrm{C} 32-\mathrm{P} 2-\mathrm{C} 22-\mathrm{C} 21$ & $99.9(5)$ \\
\hline B1-C3-C8-C7 & $176.5(5)$ & C29-P2-C22-C21 & $-150.9(5)$ \\
\hline N1-B1-C9-C10 & $39.8(7)$ & Pd1-P2-C22-C21 & $-30.4(5)$ \\
\hline C15-B1-C9-C10 & $154.4(5)$ & C21-P1-C23-C24 & $-61.2(5)$ \\
\hline C3-B1-C9-C10 & $-77.5(6)$ & C26-P1-C23-C24 & $-170.2(5)$ \\
\hline N1-B1-C9-C14 & $-140.5(5)$ & Pd1-P1-C23-C24 & $60.6(5)$ \\
\hline C15-B1-C9-C14 & $-25.8(7)$ & $\mathrm{C} 21-\mathrm{P} 1-\mathrm{C} 23-\mathrm{C} 25$ & $176.8(5)$ \\
\hline C3-B1-C9-C14 & $102.3(6)$ & C26-P1-C23-C25 & $67.8(5)$ \\
\hline C14-C9-C10-C11 & $0.5(8)$ & Pd1-P1-C23-C25 & $-61.4(5)$ \\
\hline B1-C9-C10-C11 & $-179.7(5)$ & C21-P1-C26-C28 & $-49.5(5)$ \\
\hline C9-C10-C11-C12 & $0.2(9)$ & C23-P1-C26-C28 & $60.8(6)$ \\
\hline C10-C11-C12-C13 & $0.4(9)$ & Pd1-P1-C26-C28 & $-166.6(4)$ \\
\hline C11-C12-C13-C14 & $-1.6(10)$ & C21-P1-C26-C27 & $77.8(5)$ \\
\hline
\end{tabular}




\begin{tabular}{|c|c|c|c|}
\hline C23-P1-C26-C27 & $-172.0(5)$ & C66-P4-C63-C65 & $-57.6(12)$ \\
\hline Pd1-P1-C26-C27 & $-39.3(5)$ & C56-P4-C63-C65 & $47.6(12)$ \\
\hline C22-P2-C29-C30 & $71.8(5)$ & Pd2-P4-C63-C65 & $169.2(9)$ \\
\hline C32-P2-C29-C30 & $-179.7(5)$ & C63-P4-C66-C67 & $-175.3(14)$ \\
\hline Pd1-P2-C29-C30 & $-43.1(5)$ & C56-P4-C66-C67 & $76.2(15)$ \\
\hline C22-P2-C29-C31 & $-56.0(6)$ & Pd2-P4-C66-C67 & $-38.6(15)$ \\
\hline C32-P2-C29-C31 & $52.6(6)$ & C63-P4-C66-C68 & $-50.0(12)$ \\
\hline Pd1-P2-C29-C31 & $-170.8(5)$ & C56-P4-C66-C68 & $-158.5(10)$ \\
\hline C22-P2-C32-C33 & $-64.4(5)$ & Pd2-P4-C66-C68 & $86.8(11)$ \\
\hline C29-P2-C32-C33 & $-173.0(5)$ & C57'-P3'-C55'-C56' & $-90(2)$ \\
\hline Pd1-P2-C32-C33 & $55.1(5)$ & C60'-P3'-C55'-C56' & $142(2)$ \\
\hline C22-P2-C32-C34 & $172.8(4)$ & Pd2-P3'-C55'-C56' & $24(2)$ \\
\hline C29-P2-C32-C34 & $64.2(5)$ & P3'-C55'-C56'-P4' & $-47(3)$ \\
\hline Pd1-P2-C32-C34 & $-67.7(5)$ & C55'-P3'-C57'-C58' & $58(2)$ \\
\hline C57-P3-C55-C56 & $-103.6(13)$ & C60'-P3'-C57'-C58' & 174.4(16) \\
\hline C60-P3-C55-C56 & $161.4(12)$ & Pd2-P3'-C57'-C58' & $-54(2)$ \\
\hline Pd2-P3-C55-C56 & $28.0(15)$ & C55'-P3'-C57'-C59' & $-176.8(17)$ \\
\hline P3-C55-C56-P4 & $-27.3(18)$ & C60'-P3'-C57'-C59' & $-60(2)$ \\
\hline C55-C56-P4-C63 & $145.5(12)$ & Pd2-P3'-C57'-C59' & $71.2(19)$ \\
\hline C55-C56-P4-C66 & $-105.4(14)$ & C57'-P3'-C60'-C61' & $172.9(18)$ \\
\hline C55-C56-P4-Pd2 & $15.0(14)$ & C55'-P3'-C60'-C61' & $-70.1(19)$ \\
\hline C60-P3-C57-C58 & 173.1(10) & Pd2-P3'-C60'-C61' & $42(2)$ \\
\hline C55-P3-C57-C58 & $68.8(11)$ & C57'-P3'-C60'-C62' & $-54(2)$ \\
\hline Pd2-P3-C57-C58 & $-58.3(12)$ & C55'-P3'-C60'-C62' & $63.4(17)$ \\
\hline C60-P3-C57-C59 & $-63.7(9)$ & Pd2-P3'-C60'-C62' & $176.0(13)$ \\
\hline C55-P3-C57-C59 & $-168.0(9)$ & C66'-P4'-C63'-C65' & $57(2)$ \\
\hline Pd2-P3-C57-C59 & $64.9(10)$ & C56'-P4'-C63'-C65' & $-178.6(19)$ \\
\hline C57-P3-C60-C61 & $161.8(10)$ & Pd2-P4'-C63'-C65' & $-76.1(19)$ \\
\hline C55-P3-C60-C61 & $-90.0(11)$ & C66'-P4'-C63'-C64' & $174(2)$ \\
\hline Pd2-P3-C60-C61 & $36.3(13)$ & C56'-P4'-C63'-C64' & $-62(2)$ \\
\hline C57-P3-C60-C62 & $-68.8(11)$ & Pd2-P4'-C63'-C64' & $41(2)$ \\
\hline C55-P3-C60-C62 & $39.4(13)$ & C63'-P4'-C66'-C68' & $48(3)$ \\
\hline Pd2-P3-C60-C62 & $165.7(9)$ & C56'-P4'-C66'-C68' & $-52(3)$ \\
\hline C66-P4-C63-C64 & $173.8(11)$ & Pd2-P4'-C66'-C68' & $-173(2)$ \\
\hline C56-P4-C63-C64 & $-81.1(12)$ & C63'-P4'-C66'-C67' & $172(2)$ \\
\hline Pd2-P4-C63-C64 & $40.6(13)$ & C56'-P4'-C66'-C67' & $72(2)$ \\
\hline
\end{tabular}




\begin{tabular}{|c|c|c|c|}
\hline Pd2-P4'-C66'-C67' & $-49(2)$ & $\mathrm{C} 48-\mathrm{C} 43-\mathrm{C} 44-\mathrm{C} 45$ & $-0.7(8)$ \\
\hline C36'-C35'-N2-B2 & $4.4(9)$ & B2-C43-C44-C45 & $176.0(5)$ \\
\hline $\mathrm{Pd} 2-\mathrm{C} 35$ '-N2-B2 & $-175.5(8)$ & $\mathrm{C} 43-\mathrm{C} 44-\mathrm{C} 45-\mathrm{C} 46$ & $0.7(9)$ \\
\hline C36'-C35'-N2-Pd2 & $180.0(3)$ & $\mathrm{C} 44-\mathrm{C} 45-\mathrm{C} 46-\mathrm{C} 47$ & $-0.2(9)$ \\
\hline C36-C35-N2-B2 & $-11.4(9)$ & $\mathrm{C} 45-\mathrm{C} 46-\mathrm{C} 47-\mathrm{C} 48$ & $-0.3(9)$ \\
\hline Pd2-C35-N2-B2 & $168.5(8)$ & $\mathrm{C} 46-\mathrm{C} 47-\mathrm{C} 48-\mathrm{C} 43$ & $0.3(9)$ \\
\hline C36-C35-N2-Pd2 & $-179.9(2)$ & $\mathrm{C} 44-\mathrm{C} 43-\mathrm{C} 48-\mathrm{C} 47$ & $0.1(8)$ \\
\hline C35'-N2-B2-C49 & $-142.8(9)$ & B2-C43-C48-C47 & $-176.7(5)$ \\
\hline C35-N2-B2-C49 & $-115.4(9)$ & N2-B2-C49-C54 & $-137.8(5)$ \\
\hline Pd2-N2-B2-C49 & $45.0(8)$ & C43-B2-C49-C54 & $-21.1(7)$ \\
\hline C35'-N2-B2-C43 & $96.9(9)$ & C37-B2-C49-C54 & $108.4(6)$ \\
\hline C35-N2-B2-C43 & $124.4(8)$ & $\mathrm{N} 2-\mathrm{B} 2-\mathrm{C} 49-\mathrm{C} 50$ & $41.2(6)$ \\
\hline Pd2-N2-B2-C43 & $-75.3(8)$ & C43-B2-C49-C50 & $157.9(5)$ \\
\hline C35'-N2-B2-C37 & $-22.9(11)$ & C37-B2-C49-C50 & $-72.6(6)$ \\
\hline C35-N2-B2-C37 & $4.5(11)$ & C54-C49-C50-C51 & $-2.0(8)$ \\
\hline Pd2-N2-B2-C37 & $164.9(5)$ & B2-C49-C50-C51 & $179.0(5)$ \\
\hline N2-B2-C37-C42 & $-87.6(6)$ & $\mathrm{C} 49-\mathrm{C} 50-\mathrm{C} 51-\mathrm{C} 52$ & $0.7(9)$ \\
\hline C49-B2-C37-C42 & $27.3(7)$ & C50-C51-C52-C53 & $1.6(9)$ \\
\hline C43-B2-C37-C42 & $156.6(5)$ & C51-C52-C53-C54 & $-2.6(10)$ \\
\hline N2-B2-C37-C38 & $86.4(6)$ & C52-C53-C54-C49 & $1.3(10)$ \\
\hline C49-B2-C37-C38 & $-158.7(5)$ & C50-C49-C54-C53 & $1.0(9)$ \\
\hline C43-B2-C37-C38 & $-29.4(7)$ & B2-C49-C54-C53 & $-180.0(6)$ \\
\hline C42-C37-C38-C39 & $2.7(8)$ & & \\
\hline B2-C37-C38-C39 & $-171.6(5)$ & & \\
\hline C37-C38-C39-C40 & $-1.1(9)$ & & \\
\hline C38-C39-C40-C41 & $-0.6(9)$ & & \\
\hline C39-C40-C41-C42 & $0.5(9)$ & & \\
\hline C38-C37-C42-C41 & $-2.8(8)$ & & \\
\hline B2-C37-C42-C41 & $171.6(5)$ & & \\
\hline C40-C41-C42-C37 & $1.3(9)$ & & \\
\hline N2-B2-C43-C44 & $22.7(7)$ & & \\
\hline C49-B2-C43-C44 & $-93.4(6)$ & & \\
\hline C37-B2-C43-C44 & $136.8(5)$ & & \\
\hline N2-B2-C43-C48 & $-160.7(5)$ & & \\
\hline C49-B2-C43-C48 & $83.1(6)$ & & \\
\hline C37-B2-C43-C48 & $-46.6(7)$ & & \\
\hline
\end{tabular}


REFERENCE NUMBER: jonlm38 (3a')

CRYSTAL STRUCTURE REPORT

$\mathrm{C}_{34} \mathrm{H}_{50}$ B N P 2 Pd

Report prepared for:

L. Munjanja, Prof. W. Jones

March 08, 2016

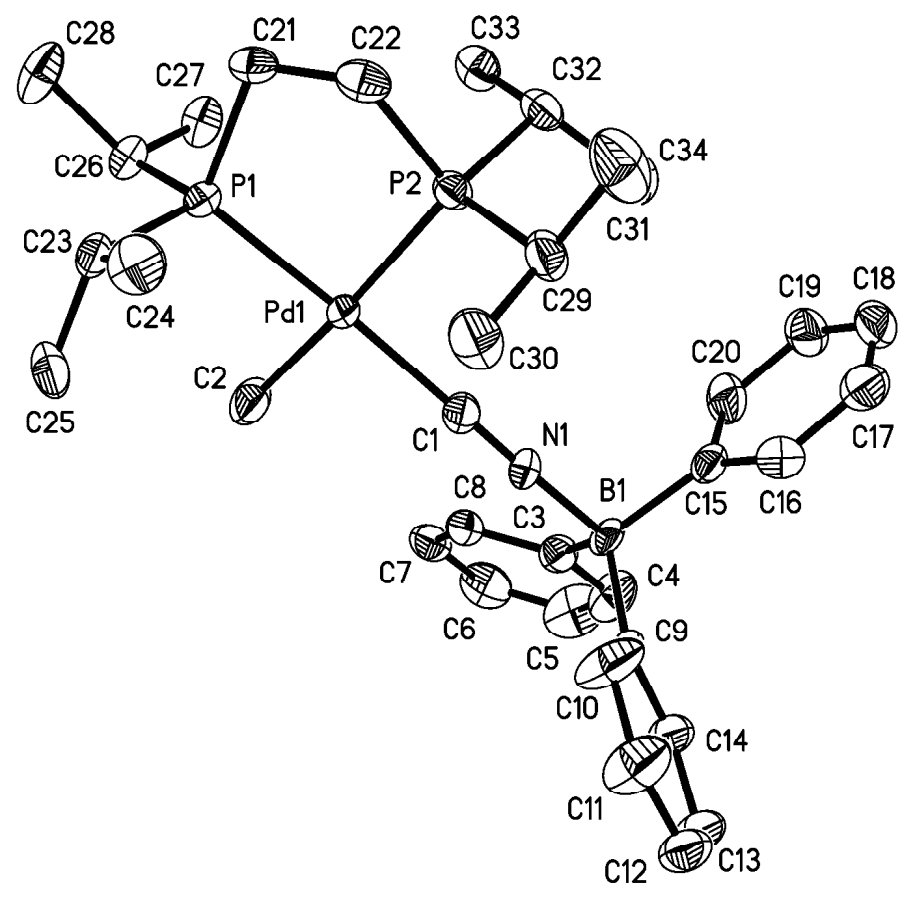

William W. Brennessel

X-ray Crystallographic Facility

Department of Chemistry, University of Rochester

120 Trustee Road

Rochester, NY 14627 


\section{Data collection}

A crystal $\left(0.42 \times 0.18 \times 0.16 \mathrm{~mm}^{3}\right)$ was placed onto the tip of a thin glass optical fiber and mounted on a Bruker SMART APEX II CCD platform diffractometer for a data collection at 100.0(5) K. ${ }^{1}$ A preliminary set of cell constants and an orientation matrix were calculated from reflections harvested from three orthogonal wedges of reciprocal space. The full data collection was carried out using MoKa radiation (graphite monochromator) with a frame time of 45 seconds and a detector distance of $4.01 \mathrm{~cm}$. A randomly oriented region of reciprocal space was surveyed: four major sections of frames were collected with $0.50^{\circ}$ steps in $\omega$ at four different $\phi$ settings and a detector position of $-38^{\circ}$ in $2 \theta$. The intensity data were corrected for absorption. ${ }^{2}$ Final cell constants were calculated from the xyz centroids of 3944 strong reflections from the actual data collection after integration. ${ }^{3}$ See Table S19 for additional crystal and refinement information.

\section{Structure solution and refinement}

The structure was solved using SHELXT-2014/5 ${ }^{4}$ and refined using SHELXL-2014/7..$^{5}$ The space group Pna2 $2_{1}$ was determined based on systematic absences and intensity statistics. A direct-methods solution was calculated which provided most non-hydrogen atoms from the E-map. Full-matrix least squares / difference Fourier cycles were performed which located the remaining non-hydrogen atoms. All non-hydrogen atoms were refined with anisotropic displacement parameters. All hydrogen atoms were placed in ideal positions and refined as riding atoms with relative isotropic displacement parameters. Although the two molecules are mostly related by pseudosymmetry, parameter correlation effects do not appear to have caused any significant problems. The crystal was an inversion twin whose final refined component mass ratio is 0.69:0.31. The final full matrix least squares refinement converged to $R 1=0.0523\left(F^{2}, I>2 \sigma(I)\right)$ and $w R 2=0.1055\left(F^{2}\right.$, all data).

\section{Structure description}

The structure is the one suggested. The asymmetric unit contains two independent palladium complexes in general positions.

Unless noted otherwise all structural diagrams containing thermal displacement ellipsoids are drawn at the $50 \%$ probability level.

Data collection, structure solution, and structure refinement were conducted at the X-ray Crystallographic Facility, B51 Hutchison Hall, Department of Chemistry, University of Rochester. All publications arising from this report MUST either 1) include William W. Brennessel as a coauthor or 2) acknowledge William W. Brennessel and the Xray Crystallographic Facility of the Department of Chemistry at the University of Rochester. 
1 APEX3, version 2015.9-0; Bruker AXS: Madison, WI, 2015.

2 Sheldrick, G. M. SADABS, version 2014/5; J. Appl. Cryst. 2015, 48, 3-10.

3 SAINT, version 8.34A; Bruker AXS: Madison, WI, 2013.

4 Sheldrick, G. M. SHELXT-2014/5; University of Göttingen: Göttingen, Germany, 2014.

5 Sheldrick, G. M. SHELXL-2014/7; Acta. Cryst. 2015, C71, 3-8.

Some equations of interest:

$$
\begin{gathered}
R_{\text {int }}=\Sigma\left|F_{\mathrm{o}}^{2}-<F_{\mathrm{o}}^{2}>\right| / \Sigma\left|F_{\mathrm{o}}{ }^{2}\right| \\
R 1=\Sigma|| F_{\mathrm{o}}|-| F_{\mathrm{c}} \| / \Sigma\left|F_{\mathrm{o}}\right| \\
w R 2=\left[\Sigma\left[w\left(F_{\mathrm{o}}{ }^{2} F_{\mathrm{c}}{ }^{2}\right)^{2}\right] / \Sigma\left[w\left(F_{\mathrm{o}}{ }^{2}\right)^{2}\right]\right]^{1 / 2} \\
\text { where } w=1 /\left[\sigma^{2}\left(F_{\mathrm{o}}{ }^{2}\right)+(a P)^{2}+b P\right] \text { and } \\
P=1 / 3 \max \left(0, F_{\mathrm{o}}{ }^{2}\right)+2 / 3 F_{\mathrm{c}}{ }^{2} \\
\mathrm{GOF}=S=\left[\Sigma\left[w\left(F_{\mathrm{o}}{ }^{2}-F_{\mathrm{c}}{ }^{2}\right)^{2}\right] /(m-n)\right]^{1 / 2}
\end{gathered}
$$

where $m=$ number of reflections and $n=$ number of parameters

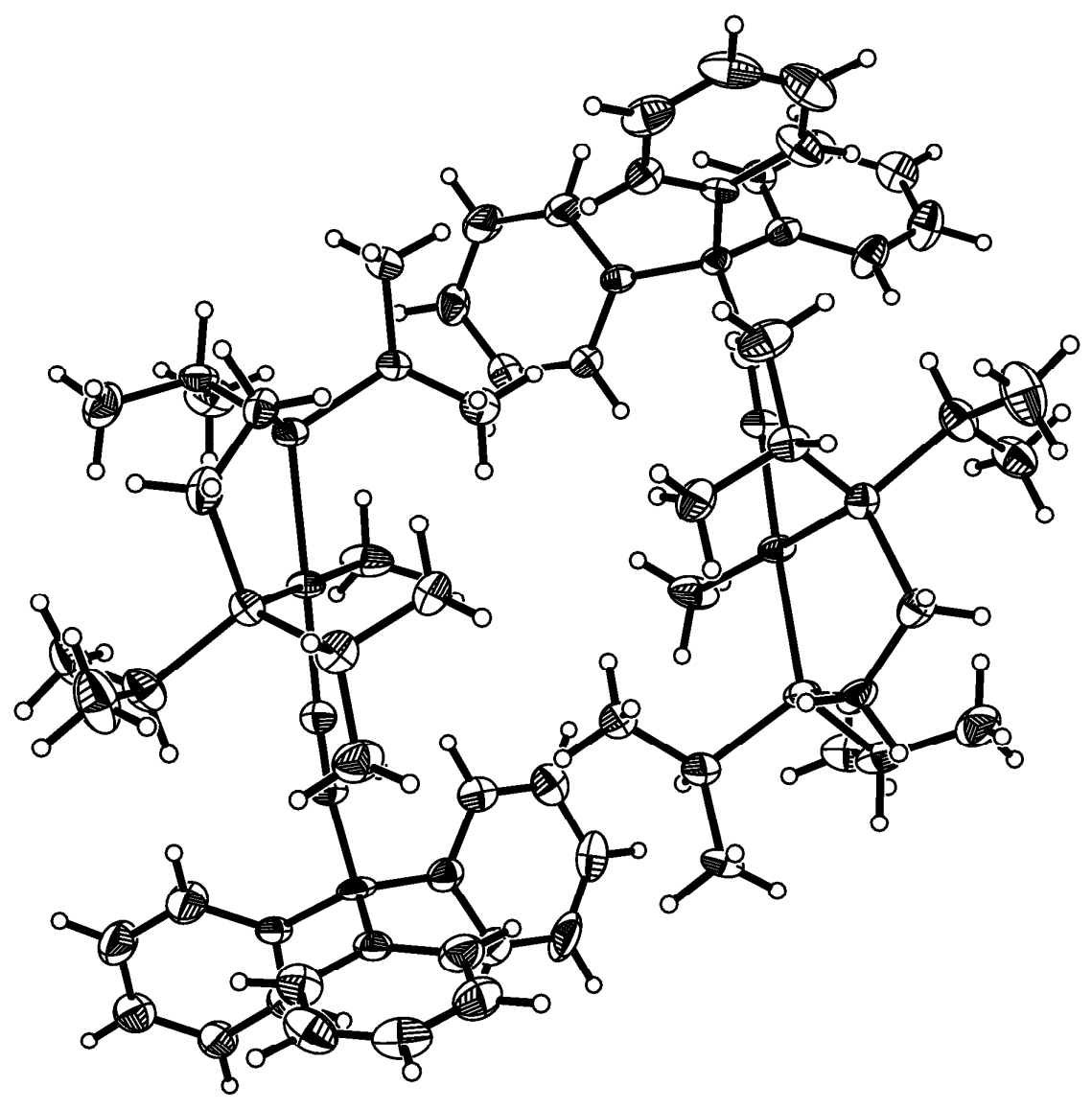




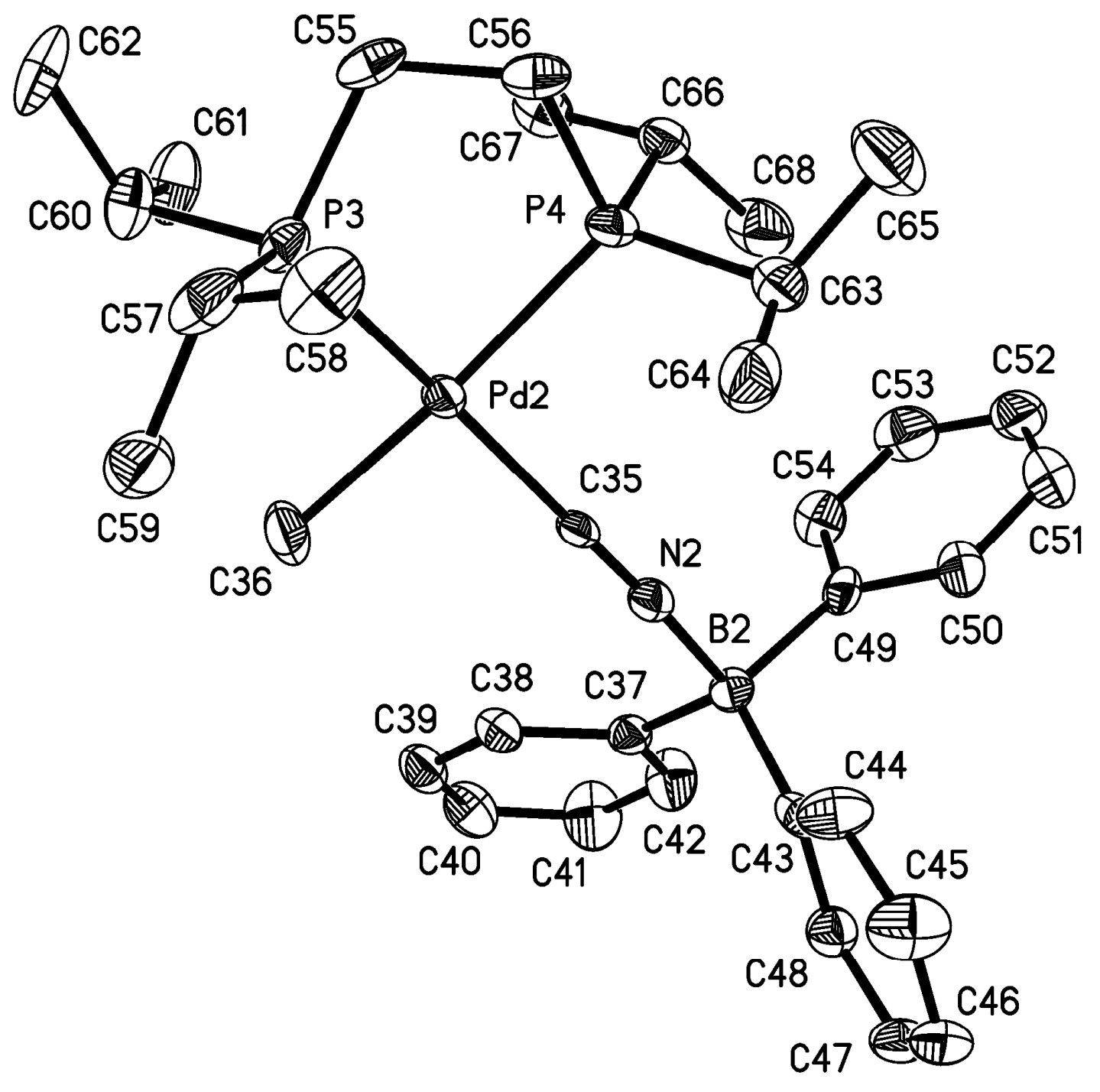


Table S19. Crystal data and structure refinement for jonlm38 (3a').

\begin{tabular}{|c|c|c|}
\hline Identification code & \multicolumn{2}{|l|}{ jonlm 38} \\
\hline Empirical formula & \multicolumn{2}{|l|}{ C34 H50 B N P2 Pd } \\
\hline Formula weight & \multicolumn{2}{|l|}{651.90} \\
\hline Temperature & \multicolumn{2}{|l|}{$100.0(5) \mathrm{K}$} \\
\hline Wavelength & \multicolumn{2}{|l|}{$0.71073 \AA$} \\
\hline Crystal system & \multicolumn{2}{|l|}{ orthorhombic } \\
\hline Space group & \multicolumn{2}{|l|}{$\operatorname{Pna2}_{1}$} \\
\hline \multirow[t]{3}{*}{ Unit cell dimensions } & $a=16.9464(15) \AA$ & $\alpha=90^{\circ}$ \\
\hline & $b=13.0266(11) \AA$ & $\beta=90^{\circ}$ \\
\hline & $c=30.638(3) \AA$ & $\gamma=90^{\circ}$ \\
\hline Volume & \multicolumn{2}{|l|}{$6763.5(10) \AA^{3}$} \\
\hline$Z$ & \multicolumn{2}{|l|}{8} \\
\hline Density (calculated) & \multicolumn{2}{|l|}{$1.280 \mathrm{Mg} / \mathrm{m}^{3}$} \\
\hline Absorption coefficient & \multicolumn{2}{|l|}{$0.666 \mathrm{~mm}^{-1}$} \\
\hline$F(000)$ & \multicolumn{2}{|l|}{2736} \\
\hline Crystal color, morphology & \multicolumn{2}{|l|}{ pale yellow, needle } \\
\hline Crystal size & \multicolumn{2}{|c|}{$0.42 \times 0.18 \times 0.16 \mathrm{~mm}^{3}$} \\
\hline Theta range for data collection & \multicolumn{2}{|l|}{1.699 to $31.517^{\circ}$} \\
\hline Index ranges & \multicolumn{2}{|c|}{$-24 \leq h \leq 24,-19 \leq k \leq 19,-45 \leq l \leq 45$} \\
\hline Reflections collected & \multicolumn{2}{|l|}{112743} \\
\hline Independent reflections & \multicolumn{2}{|c|}{$22422[R($ int $)=0.0895]$} \\
\hline Observed reflections & \multicolumn{2}{|l|}{16524} \\
\hline Completeness to theta $=31.506^{\circ}$ & \multicolumn{2}{|l|}{$99.7 \%$} \\
\hline Absorption correction & \multicolumn{2}{|l|}{ Multi-scan } \\
\hline Max. and min. transmission & \multicolumn{2}{|l|}{0.7462 and 0.6670} \\
\hline Refinement method & \multicolumn{2}{|c|}{ Full-matrix least-squares on $F^{2}$} \\
\hline Data / restraints / parameters & \multicolumn{2}{|l|}{$22422 / 1 / 722$} \\
\hline Goodness-of-fit on $F^{2}$ & \multicolumn{2}{|l|}{1.055} \\
\hline Final $R$ indices $[I>2 \operatorname{sigma}(I)]$ & \multicolumn{2}{|c|}{$R 1=0.0523, w R 2=0.0940$} \\
\hline$R$ indices (all data) & \multicolumn{2}{|c|}{$R 1=0.0848, w R 2=0.1055$} \\
\hline Absolute structure parameter & \multicolumn{2}{|l|}{$0.31(3)$} \\
\hline Largest diff. peak and hole & \multicolumn{2}{|c|}{0.930 and -0.976 e. $\AA^{-3}$} \\
\hline
\end{tabular}


Table S20. Atomic coordinates $\left(\mathrm{x} 10^{4}\right)$ and equivalent isotropic displacement parameters $\left(\AA^{2} \times 10^{3}\right)$ for jonlm38 (3a'). $U_{\text {eq }}$ is defined as one third of the trace of the orthogonalized $U_{i j}$ tensor.

\begin{tabular}{|c|c|c|c|c|}
\hline & $\mathrm{x}$ & $\mathrm{y}$ & $\mathrm{z}$ & $\mathrm{U}_{\mathrm{eq}}$ \\
\hline Pd1 & $6705(1)$ & $6764(1)$ & $3917(1)$ & $22(1)$ \\
\hline P1 & $7025(1)$ & $5135(1)$ & $4096(1)$ & $23(1)$ \\
\hline P2 & $7929(1)$ & $6880(1)$ & $3594(1)$ & $24(1)$ \\
\hline N1 & $6178(3)$ & $9026(4)$ & $3677(2)$ & $26(1)$ \\
\hline B1 & $5918(4)$ & $10185(5)$ & $3639(3)$ & $28(2)$ \\
\hline $\mathrm{C} 1$ & 6375(3) & $8203(5)$ & $3752(2)$ & $26(1)$ \\
\hline $\mathrm{C} 2$ & $5656(4)$ & 6661(6) & $4270(3)$ & $42(2)$ \\
\hline C3 & $5427(4)$ & $10419(5)$ & $4084(2)$ & $30(1)$ \\
\hline $\mathrm{C} 4$ & $5187(5)$ & $11425(6)$ & $4186(3)$ & $46(2)$ \\
\hline C5 & $4745(5)$ & $11654(6)$ & $4562(3)$ & $55(2)$ \\
\hline C6 & $4545(4)$ & $10886(6)$ & $4851(3)$ & $45(2)$ \\
\hline C7 & $4790(4)$ & $9889(6)$ & $4767(2)$ & $35(2)$ \\
\hline $\mathrm{C} 8$ & $5212(4)$ & $9679(5)$ & $4388(2)$ & $31(1)$ \\
\hline C9 & $5393(3)$ & $10309(4)$ & $3193(2)$ & $25(1)$ \\
\hline $\mathrm{C} 10$ & $5447(5)$ & $9640(6)$ & $2843(3)$ & $53(2)$ \\
\hline $\mathrm{C} 11$ & $5046(6)$ & 9794(6) & $2451(3)$ & $55(2)$ \\
\hline $\mathrm{C} 12$ & $4578(4)$ & $10645(6)$ & $2395(3)$ & $41(2)$ \\
\hline $\mathrm{C} 13$ & 4498(4) & $11307(6)$ & $2736(2)$ & $39(2)$ \\
\hline $\mathrm{C} 14$ & $4896(4)$ & $11142(5)$ & $3129(2)$ & $34(2)$ \\
\hline $\mathrm{C} 15$ & $6749(4)$ & $10824(5)$ & $3599(2)$ & $28(1)$ \\
\hline $\mathrm{C} 16$ & $7148(4)$ & $10916(5)$ & $3207(3)$ & $38(2)$ \\
\hline $\mathrm{C} 17$ & $7882(5)$ & $11372(6)$ & $3178(3)$ & $46(2)$ \\
\hline $\mathrm{C} 18$ & $8240(4)$ & 11771(6) & $3547(3)$ & $44(2)$ \\
\hline $\mathrm{C} 19$ & $7863(4)$ & $11708(5)$ & $3939(3)$ & $42(2)$ \\
\hline $\mathrm{C} 20$ & $7126(4)$ & $11240(5)$ & $3966(3)$ & $40(2)$ \\
\hline $\mathrm{C} 21$ & $8084(3)$ & 4934(5) & $3974(2)$ & $27(1)$ \\
\hline $\mathrm{C} 22$ & $8381(4)$ & $5599(5)$ & $3595(2)$ & $31(1)$ \\
\hline $\mathrm{C} 23$ & $6498(4)$ & $4110(5)$ & $3797(2)$ & $29(1)$ \\
\hline C24 & $6754(5)$ & 4076(6) & $3316(3)$ & $44(2)$ \\
\hline $\mathrm{C} 25$ & $5604(4)$ & $4210(6)$ & $3834(3)$ & $45(2)$ \\
\hline C26 & 6893(4) & 4809(5) & $4680(2)$ & $27(1)$ \\
\hline
\end{tabular}




\begin{tabular}{|c|c|c|c|c|}
\hline $\mathrm{C} 27$ & $7254(4)$ & $5629(5)$ & $4972(3)$ & $36(2)$ \\
\hline $\mathrm{C} 28$ & $7193(5)$ & $3744(5)$ & $4795(2)$ & $43(2)$ \\
\hline $\mathrm{C} 29$ & 7939(4) & $7326(6)$ & $3022(2)$ & $35(2)$ \\
\hline $\mathrm{C} 30$ & $7336(4)$ & $6705(7)$ & $2754(2)$ & $45(2)$ \\
\hline $\mathrm{C} 31$ & $8744(5)$ & $7320(8)$ & $2814(3)$ & $57(2)$ \\
\hline $\mathrm{C} 32$ & $8628(3)$ & $7720(5)$ & $3884(3)$ & $31(1)$ \\
\hline C33 & $8607(4)$ & $7489(5)$ & $4376(2)$ & $32(1)$ \\
\hline C34 & $8464(4)$ & $8869(5)$ & $3801(3)$ & $43(2)$ \\
\hline $\mathrm{Pd} 2$ & $6897(1)$ & $8286(1)$ & 6091(1) & $22(1)$ \\
\hline P3 & $7216(1)$ & 9922(1) & 5916(1) & $25(1)$ \\
\hline P4 & $8116(1)$ & $8174(1)$ & $6421(1)$ & $22(1)$ \\
\hline $\mathrm{N} 2$ & 6413(3) & $6027(4)$ & $6364(2)$ & $23(1)$ \\
\hline B2 & $6170(4)$ & $4857(5)$ & $6406(2)$ & $21(1)$ \\
\hline $\mathrm{C} 35$ & $6583(3)$ & $6856(4)$ & $6272(2)$ & $22(1)$ \\
\hline $\mathrm{C} 36$ & $5854(4)$ & $8376(6)$ & $5726(3)$ & $42(2)$ \\
\hline $\mathrm{C} 37$ & $5692(3)$ & 4579(4) & $5959(2)$ & $22(1)$ \\
\hline C38 & $5376(3)$ & $5325(5)$ & $5681(2)$ & $24(1)$ \\
\hline C39 & 4968(4) & $5061(5)$ & $5307(2)$ & $31(1)$ \\
\hline $\mathrm{C} 40$ & 4854(4) & $4052(5)$ & $5188(2)$ & $35(2)$ \\
\hline $\mathrm{C} 41$ & $5150(5)$ & $3308(6)$ & $5462(3)$ & $46(2)$ \\
\hline $\mathrm{C} 42$ & $5569(4)$ & $3562(5)$ & $5835(2)$ & $36(2)$ \\
\hline $\mathrm{C} 43$ & $5639(3)$ & $4722(4)$ & $6843(2)$ & $24(1)$ \\
\hline $\mathrm{C} 44$ & $5710(4)$ & $5379(5)$ & $7203(2)$ & $36(2)$ \\
\hline $\mathrm{C} 45$ & $5297(5)$ & 5201(7) & $7590(3)$ & $46(2)$ \\
\hline $\mathrm{C} 46$ & 4796(4) & 4392(6) & $7627(3)$ & $35(2)$ \\
\hline $\mathrm{C} 47$ & 4697(4) & $3742(5)$ & $7273(3)$ & $35(2)$ \\
\hline $\mathrm{C} 48$ & $5109(3)$ & $3912(5)$ & $6892(2)$ & $28(1)$ \\
\hline C49 & 7012(4) & $4235(4)$ & $6454(2)$ & $24(1)$ \\
\hline $\mathrm{C} 50$ & $7390(4)$ & $4140(6)$ & $6856(2)$ & $39(2)$ \\
\hline C51 & $8137(4)$ & $3700(7)$ & $6895(3)$ & $50(2)$ \\
\hline C52 & $8506(4)$ & $3311(6)$ & $6535(3)$ & $46(2)$ \\
\hline $\mathrm{C} 53$ & $8168(4)$ & $3400(5)$ & $6135(3)$ & $42(2)$ \\
\hline C54 & $7425(3)$ & $3863(4)$ & $6095(3)$ & $32(1)$ \\
\hline C55 & $8265(3)$ & $10138(5)$ & $6048(3)$ & $30(2)$ \\
\hline C56 & $8559(4)$ & $9466(5)$ & $6427(2)$ & $30(1)$ \\
\hline $\mathrm{C} 57$ & $6675(4)$ & $10929(5)$ & $6217(3)$ & $39(2)$ \\
\hline
\end{tabular}




$\begin{array}{lrrrr}\text { C58 } & 6911(5) & 10985(6) & 6692(3) & 47(2) \\ \text { C59 } & 5771(4) & 10786(6) & 6171(3) & 47(2) \\ \text { C60 } & 7120(4) & 10300(5) & 5341(2) & 33(2) \\ \text { C61 } & 7426(5) & 9453(5) & 5036(3) & 42(2) \\ \text { C62 } & 7531(5) & 11329(5) & 5245(2) & 46(2) \\ \text { C63 } & 8141(4) & 7714(6) & 6985(2) & 31(1) \\ \text { C64 } & 7525(4) & 8294(6) & 7257(2) & 41(2) \\ \text { C65 } & 8955(4) & 7740(8) & 7194(3) & 55(2) \\ \text { C66 } & 8821(3) & 7355(4) & 6121(2) & 27(1) \\ \text { C67 } & 8775(4) & 7569(5) & 5633(2) & 32(1) \\ \text { C68 } & 8686(4) & 6217(5) & 6207(3) & 42(2) \\ \end{array}$


Table S21. Bond lengths $[\AA]$ and angles $\left[^{\circ}\right]$ for jonlm38 (3a').

\begin{tabular}{|c|c|c|c|}
\hline $\operatorname{Pd}(1)-C(1)$ & $2.019(6)$ & $\mathrm{C}(11)-\mathrm{H}(11)$ & 0.9500 \\
\hline $\operatorname{Pd}(1)-C(2)$ & $2.086(7)$ & $\mathrm{C}(12)-\mathrm{C}(13)$ & $1.362(11)$ \\
\hline $\mathrm{Pd}(1)-\mathrm{P}(1)$ & $2.2593(15)$ & $\mathrm{C}(12)-\mathrm{H}(12)$ & 0.9500 \\
\hline $\mathrm{Pd}(1)-\mathrm{P}(2)$ & $2.3038(16)$ & $C(13)-C(14)$ & $1.395(10)$ \\
\hline $\mathrm{P}(1)-\mathrm{C}(23)$ & $1.849(6)$ & $\mathrm{C}(13)-\mathrm{H}(13)$ & 0.9500 \\
\hline $\mathrm{P}(1)-\mathrm{C}(26)$ & $1.850(7)$ & $\mathrm{C}(14)-\mathrm{H}(14)$ & 0.9500 \\
\hline $\mathrm{P}(1)-\mathrm{C}(21)$ & $1.852(5)$ & $C(15)-C(16)$ & $1.382(11)$ \\
\hline $\mathrm{P}(2)-\mathrm{C}(22)$ & $1.837(7)$ & $C(15)-C(20)$ & $1.402(10)$ \\
\hline $\mathrm{P}(2)-\mathrm{C}(32)$ & $1.841(6)$ & $C(16)-C(17)$ & $1.382(10)$ \\
\hline $\mathrm{P}(2)-\mathrm{C}(29)$ & $1.846(7)$ & $\mathrm{C}(16)-\mathrm{H}(16)$ & 0.9500 \\
\hline $\mathrm{N}(1)-\mathrm{C}(1)$ & $1.146(7)$ & $\mathrm{C}(17)-\mathrm{C}(18)$ & $1.384(13)$ \\
\hline $\mathrm{N}(1)-\mathrm{B}(1)$ & $1.578(8)$ & $\mathrm{C}(17)-\mathrm{H}(17)$ & 0.9500 \\
\hline $\mathrm{B}(1)-\mathrm{C}(3)$ & $1.625(11)$ & $\mathrm{C}(18)-\mathrm{C}(19)$ & $1.363(12)$ \\
\hline $\mathrm{B}(1)-\mathrm{C}(9)$ & $1.639(9)$ & $\mathrm{C}(18)-\mathrm{H}(18)$ & 0.9500 \\
\hline $\mathrm{B}(1)-\mathrm{C}(15)$ & $1.641(9)$ & $C(19)-C(20)$ & $1.392(9)$ \\
\hline $\mathrm{C}(2)-\mathrm{H}(2 \mathrm{~A})$ & 0.9800 & $\mathrm{C}(19)-\mathrm{H}(19)$ & 0.9500 \\
\hline $\mathrm{C}(2)-\mathrm{H}(2 \mathrm{~B})$ & 0.9800 & $\mathrm{C}(20)-\mathrm{H}(20)$ & 0.9500 \\
\hline $\mathrm{C}(2)-\mathrm{H}(2 \mathrm{C})$ & 0.9800 & $\mathrm{C}(21)-\mathrm{C}(22)$ & $1.535(10)$ \\
\hline $\mathrm{C}(3)-\mathrm{C}(8)$ & $1.390(9)$ & $\mathrm{C}(21)-\mathrm{H}(21 \mathrm{~A})$ & 0.9900 \\
\hline $\mathrm{C}(3)-\mathrm{C}(4)$ & $1.408(9)$ & $\mathrm{C}(21)-\mathrm{H}(21 \mathrm{~B})$ & 0.9900 \\
\hline$C(4)-C(5)$ & $1.406(12)$ & $\mathrm{C}(22)-\mathrm{H}(22 \mathrm{~A})$ & 0.9900 \\
\hline $\mathrm{C}(4)-\mathrm{H}(4)$ & 0.9500 & $\mathrm{C}(22)-\mathrm{H}(22 \mathrm{~B})$ & 0.9900 \\
\hline$C(5)-C(6)$ & $1.379(12)$ & $C(23)-C(25)$ & $1.524(9)$ \\
\hline $\mathrm{C}(5)-\mathrm{H}(5)$ & 0.9500 & $\mathrm{C}(23)-\mathrm{C}(24)$ & $1.537(11)$ \\
\hline$C(6)-C(7)$ & $1.387(10)$ & $\mathrm{C}(23)-\mathrm{H}(23)$ & 1.0000 \\
\hline $\mathrm{C}(6)-\mathrm{H}(6)$ & 0.9500 & $\mathrm{C}(24)-\mathrm{H}(24 \mathrm{~A})$ & 0.9800 \\
\hline$C(7)-C(8)$ & $1.391(10)$ & $\mathrm{C}(24)-\mathrm{H}(24 \mathrm{~B})$ & 0.9800 \\
\hline $\mathrm{C}(7)-\mathrm{H}(7)$ & 0.9500 & $\mathrm{C}(24)-\mathrm{H}(24 \mathrm{C})$ & 0.9800 \\
\hline $\mathrm{C}(8)-\mathrm{H}(8)$ & 0.9500 & $\mathrm{C}(25)-\mathrm{H}(25 \mathrm{~A})$ & 0.9800 \\
\hline$C(9)-C(10)$ & $1.384(10)$ & $\mathrm{C}(25)-\mathrm{H}(25 \mathrm{~B})$ & 0.9800 \\
\hline$C(9)-C(14)$ & $1.388(8)$ & $\mathrm{C}(25)-\mathrm{H}(25 \mathrm{C})$ & 0.9800 \\
\hline$C(10)-C(11)$ & $1.396(11)$ & $C(26)-C(28)$ & $1.519(9)$ \\
\hline $\mathrm{C}(10)-\mathrm{H}(10)$ & 0.9500 & $C(26)-C(27)$ & $1.523(9)$ \\
\hline$C(11)-C(12)$ & $1.374(11)$ & $\mathrm{C}(26)-\mathrm{H}(26)$ & 1.0000 \\
\hline
\end{tabular}




\begin{tabular}{|c|c|c|c|}
\hline $\mathrm{C}(27)-\mathrm{H}(27 \mathrm{~A})$ & 0.9800 & $\mathrm{~B}(2)-\mathrm{C}(43)$ & $1.623(9)$ \\
\hline $\mathrm{C}(27)-\mathrm{H}(27 \mathrm{~B})$ & 0.9800 & $\mathrm{~B}(2)-\mathrm{C}(37)$ & $1.631(9)$ \\
\hline $\mathrm{C}(27)-\mathrm{H}(27 \mathrm{C})$ & 0.9800 & $\mathrm{~B}(2)-\mathrm{C}(49)$ & $1.647(9)$ \\
\hline $\mathrm{C}(28)-\mathrm{H}(28 \mathrm{~A})$ & 0.9800 & $\mathrm{C}(36)-\mathrm{H}(36 \mathrm{~A})$ & 0.9800 \\
\hline $\mathrm{C}(28)-\mathrm{H}(28 \mathrm{~B})$ & 0.9800 & $\mathrm{C}(36)-\mathrm{H}(36 \mathrm{~B})$ & 0.9800 \\
\hline $\mathrm{C}(28)-\mathrm{H}(28 \mathrm{C})$ & 0.9800 & $\mathrm{C}(36)-\mathrm{H}(36 \mathrm{C})$ & 0.9800 \\
\hline $\mathrm{C}(29)-\mathrm{C}(31)$ & $1.506(10)$ & $C(37)-C(42)$ & $1.394(8)$ \\
\hline $\mathrm{C}(29)-\mathrm{C}(30)$ & $1.540(10)$ & $\mathrm{C}(37)-\mathrm{C}(38)$ & $1.397(8)$ \\
\hline $\mathrm{C}(29)-\mathrm{H}(29)$ & 1.0000 & $\mathrm{C}(38)-\mathrm{C}(39)$ & $1.381(9)$ \\
\hline $\mathrm{C}(30)-\mathrm{H}(30 \mathrm{~A})$ & 0.9800 & $\mathrm{C}(38)-\mathrm{H}(38)$ & 0.9500 \\
\hline $\mathrm{C}(30)-\mathrm{H}(30 \mathrm{~B})$ & 0.9800 & $C(39)-C(40)$ & $1.378(10)$ \\
\hline $\mathrm{C}(30)-\mathrm{H}(30 \mathrm{C})$ & 0.9800 & $\mathrm{C}(39)-\mathrm{H}(39)$ & 0.9500 \\
\hline $\mathrm{C}(31)-\mathrm{H}(31 \mathrm{~A})$ & 0.9800 & $\mathrm{C}(40)-\mathrm{C}(41)$ & $1.377(10)$ \\
\hline $\mathrm{C}(31)-\mathrm{H}(31 \mathrm{~B})$ & 0.9800 & $\mathrm{C}(40)-\mathrm{H}(40)$ & 0.9500 \\
\hline $\mathrm{C}(31)-\mathrm{H}(31 \mathrm{C})$ & 0.9800 & $\mathrm{C}(41)-\mathrm{C}(42)$ & $1.386(10)$ \\
\hline$C(32)-C(33)$ & $1.538(10)$ & $\mathrm{C}(41)-\mathrm{H}(41)$ & 0.9500 \\
\hline $\mathrm{C}(32)-\mathrm{C}(34)$ & $1.543(9)$ & $\mathrm{C}(42)-\mathrm{H}(42)$ & 0.9500 \\
\hline $\mathrm{C}(32)-\mathrm{H}(32)$ & 1.0000 & $C(43)-C(48)$ & $1.394(8)$ \\
\hline $\mathrm{C}(33)-\mathrm{H}(33 \mathrm{~A})$ & 0.9800 & $\mathrm{C}(43)-\mathrm{C}(44)$ & $1.401(9)$ \\
\hline $\mathrm{C}(33)-\mathrm{H}(33 \mathrm{~B})$ & 0.9800 & $C(44)-C(45)$ & $1.395(11)$ \\
\hline $\mathrm{C}(33)-\mathrm{H}(33 \mathrm{C})$ & 0.9800 & $\mathrm{C}(44)-\mathrm{H}(44)$ & 0.9500 \\
\hline $\mathrm{C}(34)-\mathrm{H}(34 \mathrm{~A})$ & 0.9800 & $C(45)-C(46)$ & $1.358(10)$ \\
\hline $\mathrm{C}(34)-\mathrm{H}(34 \mathrm{~B})$ & 0.9800 & $\mathrm{C}(45)-\mathrm{H}(45)$ & 0.9500 \\
\hline $\mathrm{C}(34)-\mathrm{H}(34 \mathrm{C})$ & 0.9800 & $C(46)-C(47)$ & $1.386(11)$ \\
\hline $\operatorname{Pd}(2)-C(35)$ & $2.014(5)$ & $\mathrm{C}(46)-\mathrm{H}(46)$ & 0.9500 \\
\hline $\operatorname{Pd}(2)-C(36)$ & $2.096(6)$ & $\mathrm{C}(47)-\mathrm{C}(48)$ & $1.379(10)$ \\
\hline $\mathrm{Pd}(2)-\mathrm{P}(3)$ & $2.2634(15)$ & $\mathrm{C}(47)-\mathrm{H}(47)$ & 0.9500 \\
\hline $\mathrm{Pd}(2)-\mathrm{P}(4)$ & $2.3028(15)$ & $\mathrm{C}(48)-\mathrm{H}(48)$ & 0.9500 \\
\hline$P(3)-C(60)$ & $1.836(7)$ & $C(49)-C(54)$ & $1.391(9)$ \\
\hline $\mathrm{P}(3)-\mathrm{C}(55)$ & $1.845(6)$ & $C(49)-C(50)$ & $1.393(10)$ \\
\hline $\mathrm{P}(3)-\mathrm{C}(57)$ & $1.847(7)$ & $C(50)-C(51)$ & $1.395(10)$ \\
\hline $\mathrm{P}(4)-\mathrm{C}(63)$ & $1.831(7)$ & $\mathrm{C}(50)-\mathrm{H}(50)$ & 0.9500 \\
\hline $\mathrm{P}(4)-\mathrm{C}(56)$ & $1.844(6)$ & $C(51)-C(52)$ & $1.366(12)$ \\
\hline $\mathrm{P}(4)-\mathrm{C}(66)$ & $1.847(6)$ & $\mathrm{C}(51)-\mathrm{H}(51)$ & 0.9500 \\
\hline $\mathrm{N}(2)-\mathrm{C}(35)$ & $1.153(7)$ & $C(52)-C(53)$ & $1.358(13)$ \\
\hline $\mathrm{N}(2)-\mathrm{B}(2)$ & $1.584(8)$ & $\mathrm{C}(52)-\mathrm{H}(52)$ & 0.9500 \\
\hline
\end{tabular}




\begin{tabular}{|c|c|c|c|}
\hline$C(53)-C(54)$ & $1.401(9)$ & $\mathrm{C}(66)-\mathrm{C}(68)$ & $1.523(9)$ \\
\hline $\mathrm{C}(53)-\mathrm{H}(53)$ & 0.9500 & $\mathrm{C}(66)-\mathrm{H}(66)$ & 1.0000 \\
\hline $\mathrm{C}(54)-\mathrm{H}(54)$ & 0.9500 & $\mathrm{C}(67)-\mathrm{H}(67 \mathrm{~A})$ & 0.9800 \\
\hline$C(55)-C(56)$ & $1.536(10)$ & $\mathrm{C}(67)-\mathrm{H}(67 \mathrm{~B})$ & 0.9800 \\
\hline $\mathrm{C}(55)-\mathrm{H}(55 \mathrm{~A})$ & 0.9900 & $\mathrm{C}(67)-\mathrm{H}(67 \mathrm{C})$ & 0.9800 \\
\hline $\mathrm{C}(55)-\mathrm{H}(55 \mathrm{~B})$ & 0.9900 & $\mathrm{C}(68)-\mathrm{H}(68 \mathrm{~A})$ & 0.9800 \\
\hline $\mathrm{C}(56)-\mathrm{H}(56 \mathrm{~A})$ & 0.9900 & $\mathrm{C}(68)-\mathrm{H}(68 \mathrm{~B})$ & 0.9800 \\
\hline $\mathrm{C}(56)-\mathrm{H}(56 \mathrm{~B})$ & 0.9900 & $\mathrm{C}(68)-\mathrm{H}(68 \mathrm{C})$ & 0.9800 \\
\hline$C(57)-C(58)$ & $1.511(13)$ & $\mathrm{C}(1)-\mathrm{Pd}(1)-\mathrm{C}(2)$ & $87.4(3)$ \\
\hline $\mathrm{C}(57)-\mathrm{C}(59)$ & $1.549(10)$ & $C(1)-P d(1)-P(1)$ & $177.78(17)$ \\
\hline $\mathrm{C}(57)-\mathrm{H}(57)$ & 1.0000 & $C(2)-P d(1)-P(1)$ & $91.00(19)$ \\
\hline $\mathrm{C}(58)-\mathrm{H}(58 \mathrm{~A})$ & 0.9800 & $C(1)-P d(1)-P(2)$ & $94.66(17)$ \\
\hline $\mathrm{C}(58)-\mathrm{H}(58 \mathrm{~B})$ & 0.9800 & $C(2)-P d(1)-P(2)$ & $174.1(2)$ \\
\hline $\mathrm{C}(58)-\mathrm{H}(58 \mathrm{C})$ & 0.9800 & $\mathrm{P}(1)-\mathrm{Pd}(1)-\mathrm{P}(2)$ & $87.12(6)$ \\
\hline $\mathrm{C}(59)-\mathrm{H}(59 \mathrm{~A})$ & 0.9800 & $\mathrm{C}(23)-\mathrm{P}(1)-\mathrm{C}(26)$ & $104.8(3)$ \\
\hline $\mathrm{C}(59)-\mathrm{H}(59 \mathrm{~B})$ & 0.9800 & $\mathrm{C}(23)-\mathrm{P}(1)-\mathrm{C}(21)$ & $105.4(3)$ \\
\hline $\mathrm{C}(59)-\mathrm{H}(59 \mathrm{C})$ & 0.9800 & $\mathrm{C}(26)-\mathrm{P}(1)-\mathrm{C}(21)$ & $106.2(3)$ \\
\hline$C(60)-C(61)$ & $1.536(10)$ & $\mathrm{C}(23)-\mathrm{P}(1)-\mathrm{Pd}(1)$ & $116.2(2)$ \\
\hline$C(60)-C(62)$ & $1.539(10)$ & $\mathrm{C}(26)-\mathrm{P}(1)-\mathrm{Pd}(1)$ & $115.0(2)$ \\
\hline $\mathrm{C}(60)-\mathrm{H}(60)$ & 1.0000 & $\mathrm{C}(21)-\mathrm{P}(1)-\mathrm{Pd}(1)$ & $108.5(2)$ \\
\hline $\mathrm{C}(61)-\mathrm{H}(61 \mathrm{~A})$ & 0.9800 & $\mathrm{C}(22)-\mathrm{P}(2)-\mathrm{C}(32)$ & $105.7(3)$ \\
\hline $\mathrm{C}(61)-\mathrm{H}(61 \mathrm{~B})$ & 0.9800 & $\mathrm{C}(22)-\mathrm{P}(2)-\mathrm{C}(29)$ & $106.5(3)$ \\
\hline $\mathrm{C}(61)-\mathrm{H}(61 \mathrm{C})$ & 0.9800 & $\mathrm{C}(32)-\mathrm{P}(2)-\mathrm{C}(29)$ & $105.4(3)$ \\
\hline $\mathrm{C}(62)-\mathrm{H}(62 \mathrm{~A})$ & 0.9800 & $\mathrm{C}(22)-\mathrm{P}(2)-\mathrm{Pd}(1)$ & $108.4(2)$ \\
\hline $\mathrm{C}(62)-\mathrm{H}(62 \mathrm{~B})$ & 0.9800 & $\mathrm{C}(32)-\mathrm{P}(2)-\mathrm{Pd}(1)$ & $114.3(2)$ \\
\hline $\mathrm{C}(62)-\mathrm{H}(62 \mathrm{C})$ & 0.9800 & $\mathrm{C}(29)-\mathrm{P}(2)-\mathrm{Pd}(1)$ & $115.8(2)$ \\
\hline$C(63)-C(65)$ & $1.522(9)$ & $\mathrm{C}(1)-\mathrm{N}(1)-\mathrm{B}(1)$ & $172.6(6)$ \\
\hline$C(63)-C(64)$ & $1.533(10)$ & $\mathrm{N}(1)-\mathrm{B}(1)-\mathrm{C}(3)$ & $105.1(5)$ \\
\hline $\mathrm{C}(63)-\mathrm{H}(63)$ & 1.0000 & $\mathrm{~N}(1)-\mathrm{B}(1)-\mathrm{C}(9)$ & $107.9(5)$ \\
\hline $\mathrm{C}(64)-\mathrm{H}(64 \mathrm{~A})$ & 0.9800 & $\mathrm{C}(3)-\mathrm{B}(1)-\mathrm{C}(9)$ & $113.8(5)$ \\
\hline $\mathrm{C}(64)-\mathrm{H}(64 \mathrm{~B})$ & 0.9800 & $\mathrm{~N}(1)-\mathrm{B}(1)-\mathrm{C}(15)$ & $104.6(5)$ \\
\hline $\mathrm{C}(64)-\mathrm{H}(64 \mathrm{C})$ & 0.9800 & $\mathrm{C}(3)-\mathrm{B}(1)-\mathrm{C}(15)$ & $114.0(6)$ \\
\hline $\mathrm{C}(65)-\mathrm{H}(65 \mathrm{~A})$ & 0.9800 & $\mathrm{C}(9)-\mathrm{B}(1)-\mathrm{C}(15)$ & $110.7(5)$ \\
\hline $\mathrm{C}(65)-\mathrm{H}(65 \mathrm{~B})$ & 0.9800 & $\mathrm{~N}(1)-\mathrm{C}(1)-\mathrm{Pd}(1)$ & $177.0(5)$ \\
\hline $\mathrm{C}(65)-\mathrm{H}(65 \mathrm{C})$ & 0.9800 & $\operatorname{Pd}(1)-C(2)-H(2 A)$ & 109.5 \\
\hline$C(66)-C(67)$ & $1.522(10)$ & $\mathrm{Pd}(1)-\mathrm{C}(2)-\mathrm{H}(2 \mathrm{~B})$ & 109.5 \\
\hline
\end{tabular}




\begin{tabular}{|c|c|c|c|}
\hline $\mathrm{H}(2 \mathrm{~A})-\mathrm{C}(2)-\mathrm{H}(2 \mathrm{~B})$ & 109.5 & $\mathrm{C}(14)-\mathrm{C}(13)-\mathrm{H}(13)$ & 119.5 \\
\hline $\mathrm{Pd}(1)-\mathrm{C}(2)-\mathrm{H}(2 \mathrm{C})$ & 109.5 & $C(9)-C(14)-C(13)$ & $122.4(7)$ \\
\hline $\mathrm{H}(2 \mathrm{~A})-\mathrm{C}(2)-\mathrm{H}(2 \mathrm{C})$ & 109.5 & $\mathrm{C}(9)-\mathrm{C}(14)-\mathrm{H}(14)$ & 118.8 \\
\hline $\mathrm{H}(2 \mathrm{~B})-\mathrm{C}(2)-\mathrm{H}(2 \mathrm{C})$ & 109.5 & $\mathrm{C}(13)-\mathrm{C}(14)-\mathrm{H}(14)$ & 118.8 \\
\hline$C(8)-C(3)-C(4)$ & $114.8(7)$ & $C(16)-C(15)-C(20)$ & $116.1(6)$ \\
\hline $\mathrm{C}(8)-\mathrm{C}(3)-\mathrm{B}(1)$ & $124.5(6)$ & $\mathrm{C}(16)-\mathrm{C}(15)-\mathrm{B}(1)$ & $121.9(6)$ \\
\hline $\mathrm{C}(4)-\mathrm{C}(3)-\mathrm{B}(1)$ & $120.7(6)$ & $\mathrm{C}(20)-\mathrm{C}(15)-\mathrm{B}(1)$ & $121.9(7)$ \\
\hline$C(5)-C(4)-C(3)$ & $122.3(8)$ & $\mathrm{C}(17)-\mathrm{C}(16)-\mathrm{C}(15)$ & $122.2(7)$ \\
\hline $\mathrm{C}(5)-\mathrm{C}(4)-\mathrm{H}(4)$ & 118.8 & $\mathrm{C}(17)-\mathrm{C}(16)-\mathrm{H}(16)$ & 118.9 \\
\hline $\mathrm{C}(3)-\mathrm{C}(4)-\mathrm{H}(4)$ & 118.8 & $\mathrm{C}(15)-\mathrm{C}(16)-\mathrm{H}(16)$ & 118.9 \\
\hline$C(6)-C(5)-C(4)$ & $120.2(7)$ & $\mathrm{C}(16)-\mathrm{C}(17)-\mathrm{C}(18)$ & $120.2(8)$ \\
\hline $\mathrm{C}(6)-\mathrm{C}(5)-\mathrm{H}(5)$ & 119.9 & $\mathrm{C}(16)-\mathrm{C}(17)-\mathrm{H}(17)$ & 119.9 \\
\hline $\mathrm{C}(4)-\mathrm{C}(5)-\mathrm{H}(5)$ & 119.9 & $\mathrm{C}(18)-\mathrm{C}(17)-\mathrm{H}(17)$ & 119.9 \\
\hline$C(5)-C(6)-C(7)$ & $119.1(8)$ & $\mathrm{C}(19)-\mathrm{C}(18)-\mathrm{C}(17)$ & $119.4(7)$ \\
\hline $\mathrm{C}(5)-\mathrm{C}(6)-\mathrm{H}(6)$ & 120.4 & $\mathrm{C}(19)-\mathrm{C}(18)-\mathrm{H}(18)$ & 120.3 \\
\hline $\mathrm{C}(7)-\mathrm{C}(6)-\mathrm{H}(6)$ & 120.4 & $\mathrm{C}(17)-\mathrm{C}(18)-\mathrm{H}(18)$ & 120.3 \\
\hline $\mathrm{C}(6)-\mathrm{C}(7)-\mathrm{C}(8)$ & $119.6(7)$ & $\mathrm{C}(18)-\mathrm{C}(19)-\mathrm{C}(20)$ & $120.0(8)$ \\
\hline $\mathrm{C}(6)-\mathrm{C}(7)-\mathrm{H}(7)$ & 120.2 & $\mathrm{C}(18)-\mathrm{C}(19)-\mathrm{H}(19)$ & 120.0 \\
\hline $\mathrm{C}(8)-\mathrm{C}(7)-\mathrm{H}(7)$ & 120.2 & $\mathrm{C}(20)-\mathrm{C}(19)-\mathrm{H}(19)$ & 120.0 \\
\hline$C(3)-C(8)-C(7)$ & $124.0(6)$ & $C(19)-C(20)-C(15)$ & $122.0(8)$ \\
\hline $\mathrm{C}(3)-\mathrm{C}(8)-\mathrm{H}(8)$ & 118.0 & $\mathrm{C}(19)-\mathrm{C}(20)-\mathrm{H}(20)$ & 119.0 \\
\hline $\mathrm{C}(7)-\mathrm{C}(8)-\mathrm{H}(8)$ & 118.0 & $\mathrm{C}(15)-\mathrm{C}(20)-\mathrm{H}(20)$ & 119.0 \\
\hline$C(10)-C(9)-C(14)$ & $115.0(6)$ & $\mathrm{C}(22)-\mathrm{C}(21)-\mathrm{P}(1)$ & $113.0(4)$ \\
\hline $\mathrm{C}(10)-\mathrm{C}(9)-\mathrm{B}(1)$ & $123.2(6)$ & $\mathrm{C}(22)-\mathrm{C}(21)-\mathrm{H}(21 \mathrm{~A})$ & 109.0 \\
\hline $\mathrm{C}(14)-\mathrm{C}(9)-\mathrm{B}(1)$ & $121.6(6)$ & $\mathrm{P}(1)-\mathrm{C}(21)-\mathrm{H}(21 \mathrm{~A})$ & 109.0 \\
\hline$C(9)-C(10)-C(11)$ & $123.0(7)$ & $\mathrm{C}(22)-\mathrm{C}(21)-\mathrm{H}(21 \mathrm{~B})$ & 109.0 \\
\hline $\mathrm{C}(9)-\mathrm{C}(10)-\mathrm{H}(10)$ & 118.5 & $\mathrm{P}(1)-\mathrm{C}(21)-\mathrm{H}(21 \mathrm{~B})$ & 109.0 \\
\hline $\mathrm{C}(11)-\mathrm{C}(10)-\mathrm{H}(10)$ & 118.5 & $\mathrm{H}(21 \mathrm{~A})-\mathrm{C}(21)-\mathrm{H}(21 \mathrm{~B})$ & 107.8 \\
\hline$C(12)-C(11)-C(10)$ & $120.2(8)$ & $\mathrm{C}(21)-\mathrm{C}(22)-\mathrm{P}(2)$ & $112.2(4)$ \\
\hline $\mathrm{C}(12)-\mathrm{C}(11)-\mathrm{H}(11)$ & 119.9 & $\mathrm{C}(21)-\mathrm{C}(22)-\mathrm{H}(22 \mathrm{~A})$ & 109.2 \\
\hline $\mathrm{C}(10)-\mathrm{C}(11)-\mathrm{H}(11)$ & 119.9 & $\mathrm{P}(2)-\mathrm{C}(22)-\mathrm{H}(22 \mathrm{~A})$ & 109.2 \\
\hline$C(13)-C(12)-C(11)$ & $118.3(7)$ & $\mathrm{C}(21)-\mathrm{C}(22)-\mathrm{H}(22 \mathrm{~B})$ & 109.2 \\
\hline $\mathrm{C}(13)-\mathrm{C}(12)-\mathrm{H}(12)$ & 120.9 & $\mathrm{P}(2)-\mathrm{C}(22)-\mathrm{H}(22 \mathrm{~B})$ & 109.2 \\
\hline $\mathrm{C}(11)-\mathrm{C}(12)-\mathrm{H}(12)$ & 120.9 & $\mathrm{H}(22 \mathrm{~A})-\mathrm{C}(22)-\mathrm{H}(22 \mathrm{~B})$ & 107.9 \\
\hline$C(12)-C(13)-C(14)$ & $121.0(7)$ & $\mathrm{C}(25)-\mathrm{C}(23)-\mathrm{C}(24)$ & $110.8(6)$ \\
\hline $\mathrm{C}(12)-\mathrm{C}(13)-\mathrm{H}(13)$ & 119.5 & $\mathrm{C}(25)-\mathrm{C}(23)-\mathrm{P}(1)$ & $112.4(5)$ \\
\hline
\end{tabular}




\begin{tabular}{|c|c|c|c|}
\hline $\mathrm{C}(24)-\mathrm{C}(23)-\mathrm{P}(1)$ & $111.1(5)$ & $C(30)-C(29)-P(2)$ & $109.6(5)$ \\
\hline $\mathrm{C}(25)-\mathrm{C}(23)-\mathrm{H}(23)$ & 107.4 & $\mathrm{C}(31)-\mathrm{C}(29)-\mathrm{H}(29)$ & 107.0 \\
\hline $\mathrm{C}(24)-\mathrm{C}(23)-\mathrm{H}(23)$ & 107.4 & $\mathrm{C}(30)-\mathrm{C}(29)-\mathrm{H}(29)$ & 107.0 \\
\hline $\mathrm{P}(1)-\mathrm{C}(23)-\mathrm{H}(23)$ & 107.4 & $\mathrm{P}(2)-\mathrm{C}(29)-\mathrm{H}(29)$ & 107.0 \\
\hline $\mathrm{C}(23)-\mathrm{C}(24)-\mathrm{H}(24 \mathrm{~A})$ & 109.5 & $\mathrm{C}(29)-\mathrm{C}(30)-\mathrm{H}(30 \mathrm{~A})$ & 109.5 \\
\hline $\mathrm{C}(23)-\mathrm{C}(24)-\mathrm{H}(24 \mathrm{~B})$ & 109.5 & $\mathrm{C}(29)-\mathrm{C}(30)-\mathrm{H}(30 \mathrm{~B})$ & 109.5 \\
\hline $\mathrm{H}(24 \mathrm{~A})-\mathrm{C}(24)-\mathrm{H}(24 \mathrm{~B})$ & 109.5 & $\mathrm{H}(30 \mathrm{~A})-\mathrm{C}(30)-\mathrm{H}(30 \mathrm{~B})$ & 109.5 \\
\hline $\mathrm{C}(23)-\mathrm{C}(24)-\mathrm{H}(24 \mathrm{C})$ & 109.5 & $\mathrm{C}(29)-\mathrm{C}(30)-\mathrm{H}(30 \mathrm{C})$ & 109.5 \\
\hline $\mathrm{H}(24 \mathrm{~A})-\mathrm{C}(24)-\mathrm{H}(24 \mathrm{C})$ & 109.5 & $\mathrm{H}(30 \mathrm{~A})-\mathrm{C}(30)-\mathrm{H}(30 \mathrm{C})$ & 109.5 \\
\hline $\mathrm{H}(24 \mathrm{~B})-\mathrm{C}(24)-\mathrm{H}(24 \mathrm{C})$ & 109.5 & $\mathrm{H}(30 \mathrm{~B})-\mathrm{C}(30)-\mathrm{H}(30 \mathrm{C})$ & 109.5 \\
\hline $\mathrm{C}(23)-\mathrm{C}(25)-\mathrm{H}(25 \mathrm{~A})$ & 109.5 & $\mathrm{C}(29)-\mathrm{C}(31)-\mathrm{H}(31 \mathrm{~A})$ & 109.5 \\
\hline $\mathrm{C}(23)-\mathrm{C}(25)-\mathrm{H}(25 \mathrm{~B})$ & 109.5 & $\mathrm{C}(29)-\mathrm{C}(31)-\mathrm{H}(31 \mathrm{~B})$ & 109.5 \\
\hline $\mathrm{H}(25 \mathrm{~A})-\mathrm{C}(25)-\mathrm{H}(25 \mathrm{~B})$ & 109.5 & $\mathrm{H}(31 \mathrm{~A})-\mathrm{C}(31)-\mathrm{H}(31 \mathrm{~B})$ & 109.5 \\
\hline $\mathrm{C}(23)-\mathrm{C}(25)-\mathrm{H}(25 \mathrm{C})$ & 109.5 & $\mathrm{C}(29)-\mathrm{C}(31)-\mathrm{H}(31 \mathrm{C})$ & 109.5 \\
\hline $\mathrm{H}(25 \mathrm{~A})-\mathrm{C}(25)-\mathrm{H}(25 \mathrm{C})$ & 109.5 & $\mathrm{H}(31 \mathrm{~A})-\mathrm{C}(31)-\mathrm{H}(31 \mathrm{C})$ & 109.5 \\
\hline $\mathrm{H}(25 \mathrm{~B})-\mathrm{C}(25)-\mathrm{H}(25 \mathrm{C})$ & 109.5 & $\mathrm{H}(31 \mathrm{~B})-\mathrm{C}(31)-\mathrm{H}(31 \mathrm{C})$ & 109.5 \\
\hline$C(28)-C(26)-C(27)$ & $111.7(6)$ & $\mathrm{C}(33)-\mathrm{C}(32)-\mathrm{C}(34)$ & $110.3(6)$ \\
\hline $\mathrm{C}(28)-\mathrm{C}(26)-\mathrm{P}(1)$ & $113.2(5)$ & $\mathrm{C}(33)-\mathrm{C}(32)-\mathrm{P}(2)$ & $110.0(4)$ \\
\hline $\mathrm{C}(27)-\mathrm{C}(26)-\mathrm{P}(1)$ & $111.0(4)$ & $\mathrm{C}(34)-\mathrm{C}(32)-\mathrm{P}(2)$ & $112.4(5)$ \\
\hline $\mathrm{C}(28)-\mathrm{C}(26)-\mathrm{H}(26)$ & 106.8 & $\mathrm{C}(33)-\mathrm{C}(32)-\mathrm{H}(32)$ & 108.0 \\
\hline $\mathrm{C}(27)-\mathrm{C}(26)-\mathrm{H}(26)$ & 106.8 & $\mathrm{C}(34)-\mathrm{C}(32)-\mathrm{H}(32)$ & 108.0 \\
\hline $\mathrm{P}(1)-\mathrm{C}(26)-\mathrm{H}(26)$ & 106.8 & $\mathrm{P}(2)-\mathrm{C}(32)-\mathrm{H}(32)$ & 108.0 \\
\hline $\mathrm{C}(26)-\mathrm{C}(27)-\mathrm{H}(27 \mathrm{~A})$ & 109.5 & $\mathrm{C}(32)-\mathrm{C}(33)-\mathrm{H}(33 \mathrm{~A})$ & 109.5 \\
\hline $\mathrm{C}(26)-\mathrm{C}(27)-\mathrm{H}(27 \mathrm{~B})$ & 109.5 & $\mathrm{C}(32)-\mathrm{C}(33)-\mathrm{H}(33 \mathrm{~B})$ & 109.5 \\
\hline $\mathrm{H}(27 \mathrm{~A})-\mathrm{C}(27)-\mathrm{H}(27 \mathrm{~B})$ & 109.5 & $\mathrm{H}(33 \mathrm{~A})-\mathrm{C}(33)-\mathrm{H}(33 \mathrm{~B})$ & 109.5 \\
\hline $\mathrm{C}(26)-\mathrm{C}(27)-\mathrm{H}(27 \mathrm{C})$ & 109.5 & $\mathrm{C}(32)-\mathrm{C}(33)-\mathrm{H}(33 \mathrm{C})$ & 109.5 \\
\hline $\mathrm{H}(27 \mathrm{~A})-\mathrm{C}(27)-\mathrm{H}(27 \mathrm{C})$ & 109.5 & $\mathrm{H}(33 \mathrm{~A})-\mathrm{C}(33)-\mathrm{H}(33 \mathrm{C})$ & 109.5 \\
\hline $\mathrm{H}(27 \mathrm{~B})-\mathrm{C}(27)-\mathrm{H}(27 \mathrm{C})$ & 109.5 & $\mathrm{H}(33 \mathrm{~B})-\mathrm{C}(33)-\mathrm{H}(33 \mathrm{C})$ & 109.5 \\
\hline $\mathrm{C}(26)-\mathrm{C}(28)-\mathrm{H}(28 \mathrm{~A})$ & 109.5 & $\mathrm{C}(32)-\mathrm{C}(34)-\mathrm{H}(34 \mathrm{~A})$ & 109.5 \\
\hline $\mathrm{C}(26)-\mathrm{C}(28)-\mathrm{H}(28 \mathrm{~B})$ & 109.5 & $\mathrm{C}(32)-\mathrm{C}(34)-\mathrm{H}(34 \mathrm{~B})$ & 109.5 \\
\hline $\mathrm{H}(28 \mathrm{~A})-\mathrm{C}(28)-\mathrm{H}(28 \mathrm{~B})$ & 109.5 & $\mathrm{H}(34 \mathrm{~A})-\mathrm{C}(34)-\mathrm{H}(34 \mathrm{~B})$ & 109.5 \\
\hline $\mathrm{C}(26)-\mathrm{C}(28)-\mathrm{H}(28 \mathrm{C})$ & 109.5 & $\mathrm{C}(32)-\mathrm{C}(34)-\mathrm{H}(34 \mathrm{C})$ & 109.5 \\
\hline $\mathrm{H}(28 \mathrm{~A})-\mathrm{C}(28)-\mathrm{H}(28 \mathrm{C})$ & 109.5 & $\mathrm{H}(34 \mathrm{~A})-\mathrm{C}(34)-\mathrm{H}(34 \mathrm{C})$ & 109.5 \\
\hline $\mathrm{H}(28 \mathrm{~B})-\mathrm{C}(28)-\mathrm{H}(28 \mathrm{C})$ & 109.5 & $\mathrm{H}(34 \mathrm{~B})-\mathrm{C}(34)-\mathrm{H}(34 \mathrm{C})$ & 109.5 \\
\hline $\mathrm{C}(31)-\mathrm{C}(29)-\mathrm{C}(30)$ & $111.9(7)$ & $\mathrm{C}(35)-\mathrm{Pd}(2)-\mathrm{C}(36)$ & $88.6(2)$ \\
\hline $\mathrm{C}(31)-\mathrm{C}(29)-\mathrm{P}(2)$ & $114.1(5)$ & $\mathrm{C}(35)-\operatorname{Pd}(2)-\mathrm{P}(3)$ & $177.23(18)$ \\
\hline
\end{tabular}




\begin{tabular}{|c|c|c|c|}
\hline $\mathrm{C}(36)-\mathrm{Pd}(2)-\mathrm{P}(3)$ & $91.2(2)$ & $\mathrm{C}(40)-\mathrm{C}(39)-\mathrm{C}(38)$ & $121.8(6)$ \\
\hline $\mathrm{C}(35)-\mathrm{Pd}(2)-\mathrm{P}(4)$ & $93.32(15)$ & $\mathrm{C}(40)-\mathrm{C}(39)-\mathrm{H}(39)$ & 119.1 \\
\hline $\mathrm{C}(36)-\mathrm{Pd}(2)-\mathrm{P}(4)$ & $173.7(2)$ & $\mathrm{C}(38)-\mathrm{C}(39)-\mathrm{H}(39)$ & 119.1 \\
\hline $\mathrm{P}(3)-\mathrm{Pd}(2)-\mathrm{P}(4)$ & $87.11(6)$ & $\mathrm{C}(41)-\mathrm{C}(40)-\mathrm{C}(39)$ & $117.3(6)$ \\
\hline $\mathrm{C}(60)-\mathrm{P}(3)-\mathrm{C}(55)$ & $104.8(3)$ & $\mathrm{C}(41)-\mathrm{C}(40)-\mathrm{H}(40)$ & 121.3 \\
\hline $\mathrm{C}(60)-\mathrm{P}(3)-\mathrm{C}(57)$ & $104.2(3)$ & $\mathrm{C}(39)-\mathrm{C}(40)-\mathrm{H}(40)$ & 121.3 \\
\hline $\mathrm{C}(55)-\mathrm{P}(3)-\mathrm{C}(57)$ & $105.1(3)$ & $\mathrm{C}(40)-\mathrm{C}(41)-\mathrm{C}(42)$ & $121.4(7)$ \\
\hline $\mathrm{C}(60)-\mathrm{P}(3)-\operatorname{Pd}(2)$ & $117.3(2)$ & $\mathrm{C}(40)-\mathrm{C}(41)-\mathrm{H}(41)$ & 119.3 \\
\hline $\mathrm{C}(55)-\mathrm{P}(3)-\mathrm{Pd}(2)$ & $108.7(2)$ & $\mathrm{C}(42)-\mathrm{C}(41)-\mathrm{H}(41)$ & 119.3 \\
\hline $\mathrm{C}(57)-\mathrm{P}(3)-\mathrm{Pd}(2)$ & $115.6(2)$ & $\mathrm{C}(41)-\mathrm{C}(42)-\mathrm{C}(37)$ & $121.8(7)$ \\
\hline $\mathrm{C}(63)-\mathrm{P}(4)-\mathrm{C}(56)$ & $106.2(3)$ & $\mathrm{C}(41)-\mathrm{C}(42)-\mathrm{H}(42)$ & 119.1 \\
\hline $\mathrm{C}(63)-\mathrm{P}(4)-\mathrm{C}(66)$ & $105.4(3)$ & $\mathrm{C}(37)-\mathrm{C}(42)-\mathrm{H}(42)$ & 119.1 \\
\hline $\mathrm{C}(56)-\mathrm{P}(4)-\mathrm{C}(66)$ & $105.6(3)$ & $\mathrm{C}(48)-\mathrm{C}(43)-\mathrm{C}(44)$ & $115.6(6)$ \\
\hline $\mathrm{C}(63)-\mathrm{P}(4)-\mathrm{Pd}(2)$ & $117.1(2)$ & $\mathrm{C}(48)-\mathrm{C}(43)-\mathrm{B}(2)$ & $121.8(6)$ \\
\hline $\mathrm{C}(56)-\mathrm{P}(4)-\mathrm{Pd}(2)$ & $108.2(2)$ & $\mathrm{C}(44)-\mathrm{C}(43)-\mathrm{B}(2)$ & $122.5(5)$ \\
\hline $\mathrm{C}(66)-\mathrm{P}(4)-\mathrm{Pd}(2)$ & $113.6(2)$ & $C(45)-C(44)-C(43)$ & $121.6(6)$ \\
\hline $\mathrm{C}(35)-\mathrm{N}(2)-\mathrm{B}(2)$ & $170.5(6)$ & $\mathrm{C}(45)-\mathrm{C}(44)-\mathrm{H}(44)$ & 119.2 \\
\hline $\mathrm{N}(2)-\mathrm{B}(2)-\mathrm{C}(43)$ & $108.3(5)$ & $\mathrm{C}(43)-\mathrm{C}(44)-\mathrm{H}(44)$ & 119.2 \\
\hline $\mathrm{N}(2)-\mathrm{B}(2)-\mathrm{C}(37)$ & $105.9(5)$ & $C(46)-C(45)-C(44)$ & $120.9(7)$ \\
\hline $\mathrm{C}(43)-\mathrm{B}(2)-\mathrm{C}(37)$ & $113.2(5)$ & $\mathrm{C}(46)-\mathrm{C}(45)-\mathrm{H}(45)$ & 119.5 \\
\hline $\mathrm{N}(2)-\mathrm{B}(2)-\mathrm{C}(49)$ & $104.8(4)$ & $\mathrm{C}(44)-\mathrm{C}(45)-\mathrm{H}(45)$ & 119.5 \\
\hline $\mathrm{C}(43)-\mathrm{B}(2)-\mathrm{C}(49)$ & $110.7(5)$ & $C(45)-C(46)-C(47)$ & $119.0(7)$ \\
\hline $\mathrm{C}(37)-\mathrm{B}(2)-\mathrm{C}(49)$ & $113.4(5)$ & $\mathrm{C}(45)-\mathrm{C}(46)-\mathrm{H}(46)$ & 120.5 \\
\hline $\mathrm{N}(2)-\mathrm{C}(35)-\mathrm{Pd}(2)$ & $177.9(6)$ & $\mathrm{C}(47)-\mathrm{C}(46)-\mathrm{H}(46)$ & 120.5 \\
\hline $\operatorname{Pd}(2)-C(36)-H(36 A)$ & 109.5 & $\mathrm{C}(48)-\mathrm{C}(47)-\mathrm{C}(46)$ & $120.2(6)$ \\
\hline $\mathrm{Pd}(2)-\mathrm{C}(36)-\mathrm{H}(36 \mathrm{~B})$ & 109.5 & $\mathrm{C}(48)-\mathrm{C}(47)-\mathrm{H}(47)$ & 119.9 \\
\hline $\mathrm{H}(36 \mathrm{~A})-\mathrm{C}(36)-\mathrm{H}(36 \mathrm{~B})$ & 109.5 & $\mathrm{C}(46)-\mathrm{C}(47)-\mathrm{H}(47)$ & 119.9 \\
\hline $\operatorname{Pd}(2)-C(36)-H(36 C)$ & 109.5 & $\mathrm{C}(47)-\mathrm{C}(48)-\mathrm{C}(43)$ & $122.6(7)$ \\
\hline $\mathrm{H}(36 \mathrm{~A})-\mathrm{C}(36)-\mathrm{H}(36 \mathrm{C})$ & 109.5 & $\mathrm{C}(47)-\mathrm{C}(48)-\mathrm{H}(48)$ & 118.7 \\
\hline $\mathrm{H}(36 \mathrm{~B})-\mathrm{C}(36)-\mathrm{H}(36 \mathrm{C})$ & 109.5 & $\mathrm{C}(43)-\mathrm{C}(48)-\mathrm{H}(48)$ & 118.7 \\
\hline $\mathrm{C}(42)-\mathrm{C}(37)-\mathrm{C}(38)$ & $116.0(6)$ & $\mathrm{C}(54)-\mathrm{C}(49)-\mathrm{C}(50)$ & $115.9(6)$ \\
\hline $\mathrm{C}(42)-\mathrm{C}(37)-\mathrm{B}(2)$ & $120.8(5)$ & $\mathrm{C}(54)-\mathrm{C}(49)-\mathrm{B}(2)$ & $122.4(6)$ \\
\hline $\mathrm{C}(38)-\mathrm{C}(37)-\mathrm{B}(2)$ & $123.2(5)$ & $\mathrm{C}(50)-\mathrm{C}(49)-\mathrm{B}(2)$ & $121.4(6)$ \\
\hline $\mathrm{C}(39)-\mathrm{C}(38)-\mathrm{C}(37)$ & $121.6(6)$ & $\mathrm{C}(49)-\mathrm{C}(50)-\mathrm{C}(51)$ & $122.0(7)$ \\
\hline $\mathrm{C}(39)-\mathrm{C}(38)-\mathrm{H}(38)$ & 119.2 & $\mathrm{C}(49)-\mathrm{C}(50)-\mathrm{H}(50)$ & 119.0 \\
\hline $\mathrm{C}(37)-\mathrm{C}(38)-\mathrm{H}(38)$ & 119.2 & $\mathrm{C}(51)-\mathrm{C}(50)-\mathrm{H}(50)$ & 119.0 \\
\hline
\end{tabular}




\begin{tabular}{|c|c|c|c|}
\hline $\mathrm{C}(52)-\mathrm{C}(51)-\mathrm{C}(50)$ & $119.9(8)$ & $\mathrm{C}(57)-\mathrm{C}(59)-\mathrm{H}(59 \mathrm{~A})$ & 109.5 \\
\hline $\mathrm{C}(52)-\mathrm{C}(51)-\mathrm{H}(51)$ & 120.1 & $\mathrm{C}(57)-\mathrm{C}(59)-\mathrm{H}(59 \mathrm{~B})$ & 109.5 \\
\hline $\mathrm{C}(50)-\mathrm{C}(51)-\mathrm{H}(51)$ & 120.1 & $\mathrm{H}(59 \mathrm{~A})-\mathrm{C}(59)-\mathrm{H}(59 \mathrm{~B})$ & 109.5 \\
\hline $\mathrm{C}(53)-\mathrm{C}(52)-\mathrm{C}(51)$ & $120.3(7)$ & $\mathrm{C}(57)-\mathrm{C}(59)-\mathrm{H}(59 \mathrm{C})$ & 109.5 \\
\hline $\mathrm{C}(53)-\mathrm{C}(52)-\mathrm{H}(52)$ & 119.9 & $\mathrm{H}(59 \mathrm{~A})-\mathrm{C}(59)-\mathrm{H}(59 \mathrm{C})$ & 109.5 \\
\hline $\mathrm{C}(51)-\mathrm{C}(52)-\mathrm{H}(52)$ & 119.9 & H(59B)-C(59)-H(59C) & 109.5 \\
\hline $\mathrm{C}(52)-\mathrm{C}(53)-\mathrm{C}(54)$ & $119.6(8)$ & $C(61)-C(60)-C(62)$ & $110.9(6)$ \\
\hline $\mathrm{C}(52)-\mathrm{C}(53)-\mathrm{H}(53)$ & 120.2 & $\mathrm{C}(61)-\mathrm{C}(60)-\mathrm{P}(3)$ & $111.2(5)$ \\
\hline $\mathrm{C}(54)-\mathrm{C}(53)-\mathrm{H}(53)$ & 120.2 & $\mathrm{C}(62)-\mathrm{C}(60)-\mathrm{P}(3)$ & $112.2(5)$ \\
\hline$C(49)-C(54)-C(53)$ & $122.3(7)$ & $\mathrm{C}(61)-\mathrm{C}(60)-\mathrm{H}(60)$ & 107.5 \\
\hline $\mathrm{C}(49)-\mathrm{C}(54)-\mathrm{H}(54)$ & 118.9 & $\mathrm{C}(62)-\mathrm{C}(60)-\mathrm{H}(60)$ & 107.5 \\
\hline $\mathrm{C}(53)-\mathrm{C}(54)-\mathrm{H}(54)$ & 118.9 & $\mathrm{P}(3)-\mathrm{C}(60)-\mathrm{H}(60)$ & 107.5 \\
\hline $\mathrm{C}(56)-\mathrm{C}(55)-\mathrm{P}(3)$ & $113.1(4)$ & $\mathrm{C}(60)-\mathrm{C}(61)-\mathrm{H}(61 \mathrm{~A})$ & 109.5 \\
\hline $\mathrm{C}(56)-\mathrm{C}(55)-\mathrm{H}(55 \mathrm{~A})$ & 109.0 & $\mathrm{C}(60)-\mathrm{C}(61)-\mathrm{H}(61 \mathrm{~B})$ & 109.5 \\
\hline $\mathrm{P}(3)-\mathrm{C}(55)-\mathrm{H}(55 \mathrm{~A})$ & 109.0 & $\mathrm{H}(61 \mathrm{~A})-\mathrm{C}(61)-\mathrm{H}(61 \mathrm{~B})$ & 109.5 \\
\hline $\mathrm{C}(56)-\mathrm{C}(55)-\mathrm{H}(55 \mathrm{~B})$ & 109.0 & $\mathrm{C}(60)-\mathrm{C}(61)-\mathrm{H}(61 \mathrm{C})$ & 109.5 \\
\hline $\mathrm{P}(3)-\mathrm{C}(55)-\mathrm{H}(55 \mathrm{~B})$ & 109.0 & $\mathrm{H}(61 \mathrm{~A})-\mathrm{C}(61)-\mathrm{H}(61 \mathrm{C})$ & 109.5 \\
\hline $\mathrm{H}(55 \mathrm{~A})-\mathrm{C}(55)-\mathrm{H}(55 \mathrm{~B})$ & 107.8 & $\mathrm{H}(61 \mathrm{~B})-\mathrm{C}(61)-\mathrm{H}(61 \mathrm{C})$ & 109.5 \\
\hline $\mathrm{C}(55)-\mathrm{C}(56)-\mathrm{P}(4)$ & $112.3(4)$ & $\mathrm{C}(60)-\mathrm{C}(62)-\mathrm{H}(62 \mathrm{~A})$ & 109.5 \\
\hline $\mathrm{C}(55)-\mathrm{C}(56)-\mathrm{H}(56 \mathrm{~A})$ & 109.1 & $\mathrm{C}(60)-\mathrm{C}(62)-\mathrm{H}(62 \mathrm{~B})$ & 109.5 \\
\hline$P(4)-C(56)-H(56 A)$ & 109.1 & $\mathrm{H}(62 \mathrm{~A})-\mathrm{C}(62)-\mathrm{H}(62 \mathrm{~B})$ & 109.5 \\
\hline $\mathrm{C}(55)-\mathrm{C}(56)-\mathrm{H}(56 \mathrm{~B})$ & 109.1 & $\mathrm{C}(60)-\mathrm{C}(62)-\mathrm{H}(62 \mathrm{C})$ & 109.5 \\
\hline $\mathrm{P}(4)-\mathrm{C}(56)-\mathrm{H}(56 \mathrm{~B})$ & 109.1 & $\mathrm{H}(62 \mathrm{~A})-\mathrm{C}(62)-\mathrm{H}(62 \mathrm{C})$ & 109.5 \\
\hline $\mathrm{H}(56 \mathrm{~A})-\mathrm{C}(56)-\mathrm{H}(56 \mathrm{~B})$ & 107.9 & $\mathrm{H}(62 \mathrm{~B})-\mathrm{C}(62)-\mathrm{H}(62 \mathrm{C})$ & 109.5 \\
\hline $\mathrm{C}(58)-\mathrm{C}(57)-\mathrm{C}(59)$ & $110.9(6)$ & $\mathrm{C}(65)-\mathrm{C}(63)-\mathrm{C}(64)$ & $112.2(7)$ \\
\hline C(58)-C(57)-P(3) & $112.5(5)$ & $\mathrm{C}(65)-\mathrm{C}(63)-\mathrm{P}(4)$ & $114.2(5)$ \\
\hline $\mathrm{C}(59)-\mathrm{C}(57)-\mathrm{P}(3)$ & $111.1(5)$ & $\mathrm{C}(64)-\mathrm{C}(63)-\mathrm{P}(4)$ & $109.6(5)$ \\
\hline $\mathrm{C}(58)-\mathrm{C}(57)-\mathrm{H}(57)$ & 107.4 & $\mathrm{C}(65)-\mathrm{C}(63)-\mathrm{H}(63)$ & 106.8 \\
\hline $\mathrm{C}(59)-\mathrm{C}(57)-\mathrm{H}(57)$ & 107.4 & $\mathrm{C}(64)-\mathrm{C}(63)-\mathrm{H}(63)$ & 106.8 \\
\hline $\mathrm{P}(3)-\mathrm{C}(57)-\mathrm{H}(57)$ & 107.4 & $\mathrm{P}(4)-\mathrm{C}(63)-\mathrm{H}(63)$ & 106.8 \\
\hline $\mathrm{C}(57)-\mathrm{C}(58)-\mathrm{H}(58 \mathrm{~A})$ & 109.5 & $\mathrm{C}(63)-\mathrm{C}(64)-\mathrm{H}(64 \mathrm{~A})$ & 109.5 \\
\hline C(57)-C(58)-H(58B) & 109.5 & $\mathrm{C}(63)-\mathrm{C}(64)-\mathrm{H}(64 \mathrm{~B})$ & 109.5 \\
\hline $\mathrm{H}(58 \mathrm{~A})-\mathrm{C}(58)-\mathrm{H}(58 \mathrm{~B})$ & 109.5 & $\mathrm{H}(64 \mathrm{~A})-\mathrm{C}(64)-\mathrm{H}(64 \mathrm{~B})$ & 109.5 \\
\hline $\mathrm{C}(57)-\mathrm{C}(58)-\mathrm{H}(58 \mathrm{C})$ & 109.5 & $\mathrm{C}(63)-\mathrm{C}(64)-\mathrm{H}(64 \mathrm{C})$ & 109.5 \\
\hline $\mathrm{H}(58 \mathrm{~A})-\mathrm{C}(58)-\mathrm{H}(58 \mathrm{C})$ & 109.5 & $\mathrm{H}(64 \mathrm{~A})-\mathrm{C}(64)-\mathrm{H}(64 \mathrm{C})$ & 109.5 \\
\hline $\mathrm{H}(58 \mathrm{~B})-\mathrm{C}(58)-\mathrm{H}(58 \mathrm{C})$ & 109.5 & $\mathrm{H}(64 \mathrm{~B})-\mathrm{C}(64)-\mathrm{H}(64 \mathrm{C})$ & 109.5 \\
\hline
\end{tabular}




$\begin{array}{llll}\mathrm{C}(63)-\mathrm{C}(65)-\mathrm{H}(65 \mathrm{~A}) & 109.5 & \mathrm{C}(66)-\mathrm{C}(67)-\mathrm{H}(67 \mathrm{~A}) & 109.5 \\ \mathrm{C}(63)-\mathrm{C}(65)-\mathrm{H}(65 \mathrm{~B}) & 109.5 & \mathrm{C}(66)-\mathrm{C}(67)-\mathrm{H}(67 \mathrm{~B}) & 109.5 \\ \mathrm{H}(65 \mathrm{~A})-\mathrm{C}(65)-\mathrm{H}(65 \mathrm{~B}) & 109.5 & \mathrm{H}(67 \mathrm{~A})-\mathrm{C}(67)-\mathrm{H}(67 \mathrm{~B}) & 109.5 \\ \mathrm{C}(63)-\mathrm{C}(65)-\mathrm{H}(65 \mathrm{C}) & 109.5 & \mathrm{C}(66)-\mathrm{C}(67)-\mathrm{H}(67 \mathrm{C}) & 109.5 \\ \mathrm{H}(65 \mathrm{~A})-\mathrm{C}(65)-\mathrm{H}(65 \mathrm{C}) & 109.5 & \mathrm{H}(67 \mathrm{~A})-\mathrm{C}(67)-\mathrm{H}(67 \mathrm{C}) & 109.5 \\ \mathrm{H}(65 \mathrm{~B})-\mathrm{C}(65)-\mathrm{H}(65 \mathrm{C}) & 109.5 & \mathrm{H}(67 \mathrm{~B})-\mathrm{C}(67)-\mathrm{H}(67 \mathrm{C}) & 109.5 \\ \mathrm{C}(67)-\mathrm{C}(66)-\mathrm{C}(68) & 109.9(6) & \mathrm{C}(66)-\mathrm{C}(68)-\mathrm{H}(68 \mathrm{~A}) & 109.5 \\ \mathrm{C}(67)-\mathrm{C}(66)-\mathrm{P}(4) & 110.4(4) & \mathrm{C}(66)-\mathrm{C}(68)-\mathrm{H}(68 \mathrm{~B}) & 109.5 \\ \mathrm{C}(68)-\mathrm{C}(66)-\mathrm{P}(4) & 112.3(4) & \mathrm{H}(68 \mathrm{~A})-\mathrm{C}(68)-\mathrm{H}(68 \mathrm{~B}) & 109.5 \\ \mathrm{C}(67)-\mathrm{C}(66)-\mathrm{H}(66) & 108.0 & \mathrm{C}(66)-\mathrm{C}(68)-\mathrm{H}(68 \mathrm{C}) & 109.5 \\ \mathrm{C}(68)-\mathrm{C}(66)-\mathrm{H}(66) & 108.0 & \mathrm{H}(68 \mathrm{~A})-\mathrm{C}(68)-\mathrm{H}(68 \mathrm{C}) & 109.5 \\ \mathrm{P}(4)-\mathrm{C}(66)-\mathrm{H}(66) & 108.0 & \mathrm{H}(68 \mathrm{~B})-\mathrm{C}(68)-\mathrm{H}(68 \mathrm{C}) & 109.5\end{array}$


Table S22. Anisotropic displacement parameters $\left(\AA^{2} \times 10^{3}\right)$ for jonlm 38 (3a'). The anisotropic displacement factor exponent takes the form: $-2 \pi^{2}\left[\mathrm{~h}^{2} \mathrm{a}^{* 2} \mathrm{U}_{11}+\ldots+2 \mathrm{hka} \mathrm{a}^{*} \mathrm{U}_{12}\right]$

\begin{tabular}{|c|c|c|c|c|c|c|}
\hline & $\mathrm{U}_{11}$ & $\mathrm{U}_{22}$ & $\mathrm{U}_{33}$ & $\mathrm{U}_{23}$ & $\mathrm{U}_{13}$ & $\mathrm{U}_{12}$ \\
\hline Pd1 & $18(1)$ & $18(1)$ & $31(1)$ & $5(1)$ & $0(1)$ & $2(1)$ \\
\hline P1 & $23(1)$ & $20(1)$ & $28(1)$ & $2(1)$ & $-3(1)$ & $3(1)$ \\
\hline P2 & $19(1)$ & $28(1)$ & $24(1)$ & $2(1)$ & $1(1)$ & $-1(1)$ \\
\hline N1 & $29(3)$ & $18(2)$ & $31(3)$ & $5(2)$ & $-1(2)$ & $-2(2)$ \\
\hline B1 & $33(4)$ & $12(3)$ & $38(4)$ & $4(3)$ & $-10(3)$ & $1(2)$ \\
\hline $\mathrm{C} 1$ & $27(3)$ & $24(3)$ & $29(3)$ & $2(2)$ & $1(2)$ & $0(2)$ \\
\hline $\mathrm{C} 2$ & $34(4)$ & $32(4)$ & $59(5)$ & $17(3)$ & $12(3)$ & $9(3)$ \\
\hline $\mathrm{C} 3$ & $32(3)$ & $24(3)$ & $35(4)$ & $-1(2)$ & $-11(3)$ & $-3(2)$ \\
\hline $\mathrm{C} 4$ & $68(5)$ & $30(4)$ & $39(4)$ & $-5(3)$ & $-9(4)$ & $5(3)$ \\
\hline $\mathrm{C} 5$ & $79(6)$ & $42(5)$ & $45(5)$ & $-27(4)$ & $-10(4)$ & 20(4) \\
\hline C6 & $45(4)$ & $55(5)$ & $35(4)$ & $-16(3)$ & $-7(3)$ & $-4(4)$ \\
\hline $\mathrm{C} 7$ & $26(3)$ & $43(4)$ & $35(4)$ & $1(3)$ & $-5(3)$ & $-3(3)$ \\
\hline $\mathrm{C} 8$ & $25(3)$ & $28(3)$ & $39(4)$ & $2(3)$ & $-4(3)$ & $0(2)$ \\
\hline C9 & $27(3)$ & $18(3)$ & $30(3)$ & $5(2)$ & $-5(2)$ & $-1(2)$ \\
\hline $\mathrm{C} 10$ & $77(6)$ & $38(4)$ & $45(5)$ & $-6(3)$ & $-25(4)$ & $25(4)$ \\
\hline $\mathrm{C} 11$ & $85(6)$ & $38(4)$ & $43(5)$ & $-10(4)$ & $-26(4)$ & $9(4)$ \\
\hline $\mathrm{C} 12$ & $45(4)$ & $38(4)$ & $40(5)$ & $5(3)$ & $-18(3)$ & $2(3)$ \\
\hline $\mathrm{C} 13$ & $47(4)$ & $35(4)$ & $37(4)$ & $2(3)$ & $-14(3)$ & $6(3)$ \\
\hline C14 & $37(4)$ & $30(3)$ & $36(4)$ & $1(3)$ & $-10(3)$ & $2(3)$ \\
\hline $\mathrm{C} 15$ & $32(3)$ & $20(3)$ & $31(4)$ & $5(2)$ & $-2(3)$ & $3(2)$ \\
\hline $\mathrm{C} 16$ & $32(4)$ & $41(4)$ & $42(4)$ & $4(3)$ & $-5(3)$ & $3(3)$ \\
\hline $\mathrm{C} 17$ & $38(4)$ & $55(5)$ & $45(5)$ & $17(4)$ & $4(4)$ & $9(4)$ \\
\hline C18 & $29(3)$ & $37(4)$ & $68(6)$ & $10(4)$ & $10(3)$ & $2(3)$ \\
\hline C19 & $38(3)$ & $33(3)$ & $54(4)$ & $-2(4)$ & $-4(4)$ & $-7(3)$ \\
\hline $\mathrm{C} 20$ & $43(4)$ & $29(3)$ & $47(5)$ & $0(3)$ & $-2(3)$ & $-9(3)$ \\
\hline $\mathrm{C} 21$ & $22(3)$ & $30(3)$ & $29(4)$ & $-6(3)$ & $-3(2)$ & $7(2)$ \\
\hline $\mathrm{C} 22$ & $23(3)$ & $40(4)$ & $31(4)$ & $-9(3)$ & $0(3)$ & $5(2)$ \\
\hline $\mathrm{C} 23$ & $35(3)$ & $19(3)$ & $32(4)$ & $5(2)$ & $-10(3)$ & $-4(2)$ \\
\hline $\mathrm{C} 24$ & $53(5)$ & $37(4)$ & $42(5)$ & $-13(4)$ & $-12(4)$ & $-1(3)$ \\
\hline $\mathrm{C} 25$ & $42(4)$ & $38(4)$ & $56(6)$ & $12(4)$ & $-9(3)$ & $-20(3)$ \\
\hline $\mathrm{C} 26$ & $35(3)$ & $22(3)$ & $26(3)$ & $2(2)$ & $1(3)$ & $3(2)$ \\
\hline
\end{tabular}




\begin{tabular}{|c|c|c|c|c|c|c|}
\hline $\mathrm{C} 27$ & $46(4)$ & $29(3)$ & $32(4)$ & $2(3)$ & $3(3)$ & $-1(3)$ \\
\hline $\mathrm{C} 28$ & $75(5)$ & $25(3)$ & $28(4)$ & $5(3)$ & $-6(3)$ & $4(3)$ \\
\hline $\mathrm{C} 29$ & $30(3)$ & $44(4)$ & $31(4)$ & $9(3)$ & $-1(3)$ & $-11(3)$ \\
\hline $\mathrm{C} 30$ & $47(4)$ & $62(5)$ & $28(4)$ & $7(4)$ & $-9(3)$ & $-17(4)$ \\
\hline $\mathrm{C} 31$ & $52(5)$ & $85(7)$ & $33(5)$ & $3(4)$ & $5(4)$ & $-22(4)$ \\
\hline $\mathrm{C} 32$ & $21(3)$ & $34(3)$ & $37(4)$ & $-2(3)$ & $-1(3)$ & $-4(2)$ \\
\hline $\mathrm{C} 33$ & $30(3)$ & $31(3)$ & $34(4)$ & $-9(3)$ & $1(3)$ & $-2(2)$ \\
\hline C34 & $42(4)$ & $32(4)$ & $56(6)$ & $0(3)$ & $-9(3)$ & $-10(3)$ \\
\hline $\mathrm{Pd} 2$ & $17(1)$ & $16(1)$ & $33(1)$ & $4(1)$ & $-4(1)$ & $-1(1)$ \\
\hline P3 & $31(1)$ & $15(1)$ & $29(1)$ & $2(1)$ & $3(1)$ & $-1(1)$ \\
\hline P4 & $17(1)$ & $25(1)$ & $26(1)$ & $-1(1)$ & $-1(1)$ & $-5(1)$ \\
\hline $\mathrm{N} 2$ & $18(2)$ & $22(2)$ & $29(3)$ & $3(2)$ & $-4(2)$ & $-2(2)$ \\
\hline B2 & $23(3)$ & $15(3)$ & $25(3)$ & $1(2)$ & $-3(2)$ & $-5(2)$ \\
\hline $\mathrm{C} 35$ & $12(2)$ & $19(3)$ & $35(3)$ & $6(2)$ & $-5(2)$ & $-2(2)$ \\
\hline $\mathrm{C} 36$ & $32(4)$ & $36(4)$ & $59(5)$ & $17(3)$ & $-21(3)$ & $-2(3)$ \\
\hline C37 & $18(3)$ & $18(3)$ & $30(3)$ & $2(2)$ & $1(2)$ & $-2(2)$ \\
\hline $\mathrm{C} 38$ & $19(3)$ & $24(3)$ & $29(3)$ & $-2(2)$ & $-1(2)$ & $1(2)$ \\
\hline C39 & $23(3)$ & $41(4)$ & $28(3)$ & $1(3)$ & $1(2)$ & $8(3)$ \\
\hline $\mathrm{C} 40$ & $32(4)$ & $41(4)$ & $33(4)$ & $-9(3)$ & $-11(3)$ & $-1(3)$ \\
\hline C41 & $58(5)$ & $28(4)$ & $52(5)$ & $-7(3)$ & $-20(4)$ & $-11(3)$ \\
\hline $\mathrm{C} 42$ & $45(4)$ & $24(3)$ & $40(4)$ & $-3(3)$ & $-9(3)$ & $-1(3)$ \\
\hline $\mathrm{C} 43$ & $17(3)$ & $21(3)$ & $34(4)$ & $0(2)$ & $-4(2)$ & $3(2)$ \\
\hline C44 & $34(4)$ & $32(4)$ & $42(4)$ & $-11(3)$ & $8(3)$ & $-13(3)$ \\
\hline $\mathrm{C} 45$ & $44(4)$ & $53(5)$ & $40(5)$ & $-18(4)$ & $10(3)$ & $-6(4)$ \\
\hline $\mathrm{C} 46$ & $22(3)$ & $45(4)$ & $39(4)$ & $4(3)$ & $9(3)$ & $1(3)$ \\
\hline C47 & $23(3)$ & $35(4)$ & $48(5)$ & $6(3)$ & $3(3)$ & $-8(3)$ \\
\hline C48 & $26(3)$ & $27(3)$ & $32(4)$ & $1(3)$ & $-1(2)$ & $-2(2)$ \\
\hline C49 & $27(3)$ & $18(3)$ & $28(3)$ & $9(2)$ & $1(2)$ & $2(2)$ \\
\hline C50 & $28(4)$ & $54(5)$ & $35(4)$ & $16(3)$ & $1(3)$ & $7(3)$ \\
\hline C51 & $32(4)$ & $66(5)$ & $52(5)$ & $21(4)$ & $-8(4)$ & $3(4)$ \\
\hline C52 & $25(3)$ & $43(4)$ & $71(6)$ & $24(4)$ & $7(4)$ & $9(3)$ \\
\hline C53 & $36(3)$ & $31(3)$ & $58(5)$ & 1(3) & $13(4)$ & $6(3)$ \\
\hline C54 & $36(3)$ & $27(3)$ & $32(3)$ & $-1(3)$ & 1(3) & $2(2)$ \\
\hline C55 & $36(4)$ & $18(3)$ & $36(4)$ & $-2(3)$ & $9(3)$ & $-12(2)$ \\
\hline C56 & $25(3)$ & $27(3)$ & $37(4)$ & $-5(3)$ & $2(3)$ & $-11(2)$ \\
\hline C57 & $46(4)$ & $21(3)$ & $51(5)$ & $3(3)$ & $23(3)$ & $0(3)$ \\
\hline
\end{tabular}




\begin{tabular}{lllllll} 
C58 & $63(5)$ & $32(4)$ & $45(6)$ & $-8(4)$ & $16(4)$ & $4(3)$ \\
C59 & $38(4)$ & $40(4)$ & $62(6)$ & $12(4)$ & $18(4)$ & $9(3)$ \\
C60 & $46(4)$ & $23(3)$ & $31(4)$ & $3(3)$ & $-1(3)$ & $7(3)$ \\
C61 & $68(5)$ & $27(3)$ & $30(4)$ & $-1(3)$ & $-1(3)$ & $9(3)$ \\
C62 & $83(6)$ & $24(3)$ & $30(4)$ & $13(3)$ & $0(4)$ & $-1(3)$ \\
C63 & $24(3)$ & $41(4)$ & $28(4)$ & $4(3)$ & $-3(2)$ & $-2(3)$ \\
C64 & $42(4)$ & $47(4)$ & $33(4)$ & $10(3)$ & $5(3)$ & $5(3)$ \\
C65 & $33(4)$ & $90(7)$ & $41(5)$ & $0(4)$ & $-9(3)$ & $3(4)$ \\
C66 & $18(3)$ & $27(3)$ & $36(4)$ & $2(3)$ & $0(3)$ & $1(2)$ \\
C67 & $28(3)$ & $31(3)$ & $37(4)$ & $-8(3)$ & $1(3)$ & $3(2)$ \\
C68 & $29(4)$ & $28(3)$ & $67(6)$ & $-3(3)$ & $1(3)$ & $4(3)$ \\
& & & & & & \\
\hline
\end{tabular}


Table S23. Hydrogen coordinates $\left(\times 10^{4}\right)$ and isotropic displacement parameters $\left(\AA^{2} \times 10^{3}\right)$ for jonlm38 (3a').

\begin{tabular}{|c|c|c|c|c|}
\hline & $\mathrm{x}$ & $\mathrm{y}$ & $\mathrm{z}$ & $\mathrm{U}(\mathrm{eq})$ \\
\hline $\mathrm{H} 2 \mathrm{~A}$ & 5213 & 6867 & 4084 & 63 \\
\hline $\mathrm{H} 2 \mathrm{~B}$ & 5683 & 7116 & 4525 & 63 \\
\hline $\mathrm{H} 2 \mathrm{C}$ & 5579 & 5952 & 4368 & 63 \\
\hline $\mathrm{H} 4$ & 5329 & 11969 & 3995 & 55 \\
\hline H5 & 4584 & 12341 & 4618 & 66 \\
\hline H6 & 4244 & 11037 & 5105 & 54 \\
\hline $\mathrm{H} 7$ & 4670 & 9354 & 4967 & 42 \\
\hline H8 & 5363 & 8989 & 4334 & 37 \\
\hline H10 & 5772 & 9049 & 2872 & 64 \\
\hline H11 & 5097 & 9309 & 2221 & 67 \\
\hline H12 & 4317 & 10769 & 2126 & 49 \\
\hline H13 & 4165 & 11890 & 2707 & 47 \\
\hline H14 & 4824 & 11617 & 3361 & 41 \\
\hline H16 & 6908 & 10656 & 2950 & 46 \\
\hline H17 & 8142 & 11411 & 2904 & 55 \\
\hline H18 & 8744 & 12086 & 3527 & 53 \\
\hline H19 & 8103 & 11983 & 4193 & 50 \\
\hline $\mathrm{H} 20$ & 6871 & 11202 & 4242 & 48 \\
\hline $\mathrm{H} 21 \mathrm{~A}$ & 8398 & 5093 & 4238 & 32 \\
\hline $\mathrm{H} 21 \mathrm{~B}$ & 8171 & 4202 & 3902 & 32 \\
\hline $\mathrm{H} 22 \mathrm{~A}$ & 8260 & 5251 & 3315 & 38 \\
\hline H22B & 8961 & 5671 & 3616 & 38 \\
\hline $\mathrm{H} 23$ & 6650 & 3440 & 3932 & 35 \\
\hline $\mathrm{H} 24 \mathrm{~A}$ & 6483 & 3510 & 3168 & 66 \\
\hline H24B & 7326 & 3970 & 3299 & 66 \\
\hline $\mathrm{H} 24 \mathrm{C}$ & 6617 & 4726 & 3174 & 66 \\
\hline $\mathrm{H} 25 \mathrm{~A}$ & 5351 & 3666 & 3663 & 68 \\
\hline $\mathrm{H} 25 \mathrm{~B}$ & 5439 & 4881 & 3723 & 68 \\
\hline $\mathrm{H} 25 \mathrm{C}$ & 5448 & 4146 & 4141 & 68 \\
\hline $\mathrm{H} 26$ & 6312 & 4810 & 4737 & 33 \\
\hline
\end{tabular}




\begin{tabular}{|c|c|c|c|c|}
\hline $\mathrm{H} 27 \mathrm{~A}$ & 7124 & 5481 & 5278 & 53 \\
\hline $\mathrm{H} 27 \mathrm{~B}$ & 7041 & 6303 & 4892 & 53 \\
\hline $\mathrm{H} 27 \mathrm{C}$ & 7828 & 5632 & 4935 & 53 \\
\hline $\mathrm{H} 28 \mathrm{~A}$ & 7120 & 3622 & 5108 & 64 \\
\hline $\mathrm{H} 28 \mathrm{~B}$ & 7755 & 3693 & 4723 & 64 \\
\hline $\mathrm{H} 28 \mathrm{C}$ & 6898 & 3228 & 4629 & 64 \\
\hline $\mathrm{H} 29$ & 7753 & 8055 & 3023 & 42 \\
\hline $\mathrm{H} 30 \mathrm{~A}$ & 7266 & 7026 & 2467 & 68 \\
\hline H30B & 6829 & 6694 & 2908 & 68 \\
\hline $\mathrm{H} 30 \mathrm{C}$ & 7528 & 6001 & 2716 & 68 \\
\hline $\mathrm{H} 31 \mathrm{~A}$ & 8703 & 7568 & 2513 & 85 \\
\hline H31B & 8954 & 6619 & 2814 & 85 \\
\hline $\mathrm{H} 31 \mathrm{C}$ & 9098 & 7770 & 2979 & 85 \\
\hline H32 & 9171 & 7563 & 3775 & 37 \\
\hline $\mathrm{H} 33 \mathrm{~A}$ & 8983 & 7937 & 4528 & 48 \\
\hline H33B & 8752 & 6770 & 4426 & 48 \\
\hline $\mathrm{H} 33 \mathrm{C}$ & 8074 & 7613 & 4489 & 48 \\
\hline $\mathrm{H} 34 \mathrm{~A}$ & 8836 & 9285 & 3971 & 65 \\
\hline H34B & 7923 & 9032 & 3890 & 65 \\
\hline $\mathrm{H} 34 \mathrm{C}$ & 8529 & 9019 & 3490 & 65 \\
\hline $\mathrm{H} 36 \mathrm{~A}$ & 5399 & 8363 & 5923 & 63 \\
\hline H36B & 5825 & 7791 & 5526 & 63 \\
\hline $\mathrm{H} 36 \mathrm{C}$ & 5850 & 9016 & 5558 & 63 \\
\hline H38 & 5444 & 6030 & 5751 & 29 \\
\hline H39 & 4760 & 5590 & 5128 & 37 \\
\hline $\mathrm{H} 40$ & 4581 & 3877 & 4927 & 42 \\
\hline H41 & 5067 & 2605 & 5393 & 55 \\
\hline H42 & 5777 & 3028 & 6012 & 43 \\
\hline H44 & 6047 & 5960 & 7184 & 43 \\
\hline $\mathrm{H} 45$ & 5366 & 5653 & 7830 & 55 \\
\hline H46 & 4518 & 4273 & 7891 & 42 \\
\hline H47 & 4345 & 3177 & 7293 & 42 \\
\hline $\mathrm{H} 48$ & 5028 & 3460 & 6653 & 34 \\
\hline $\mathrm{H} 50$ & 7131 & 4383 & 7111 & 47 \\
\hline H51 & 8388 & 3670 & 7172 & 60 \\
\hline H52 & 9001 & 2976 & 6564 & 55 \\
\hline
\end{tabular}

S-111 


\begin{tabular}{|c|c|c|c|c|}
\hline H53 & 8433 & 3149 & 5883 & 50 \\
\hline H54 & 7196 & 3925 & 5813 & 38 \\
\hline H55A & 8588 & 9993 & 5786 & 36 \\
\hline H55B & 8341 & 10869 & 6126 & 36 \\
\hline H56A & 8430 & 9806 & 6707 & 36 \\
\hline H56B & 9140 & 9402 & 6408 & 36 \\
\hline H57 & 6813 & 11603 & 6081 & 47 \\
\hline H58A & 6598 & 11513 & 6839 & 70 \\
\hline H58B & 7473 & 11159 & 6713 & 70 \\
\hline $\mathrm{H} 58 \mathrm{C}$ & 6818 & 10318 & 6831 & 70 \\
\hline H59A & 5498 & 11345 & 6323 & 70 \\
\hline H59B & 5616 & 10128 & 6299 & 70 \\
\hline $\mathrm{H} 59 \mathrm{C}$ & 5627 & 10795 & 5861 & 70 \\
\hline H60 & 6545 & 10392 & 5279 & 40 \\
\hline H61A & 7305 & 9634 & 4733 & 62 \\
\hline H61B & 7170 & 8801 & 5110 & 62 \\
\hline H61C & 7999 & 9384 & 5071 & 62 \\
\hline H62A & 7402 & 11550 & 4948 & 69 \\
\hline H62B & 8104 & 11244 & 5272 & 69 \\
\hline $\mathrm{H} 62 \mathrm{C}$ & 7350 & 11847 & 5454 & 69 \\
\hline H63 & 7972 & 6978 & 6979 & 38 \\
\hline H64A & 7511 & 8010 & 7553 & 61 \\
\hline H64B & 7004 & 8219 & 7121 & 61 \\
\hline H64C & 7666 & 9022 & 7270 & 61 \\
\hline H65A & 8931 & 7426 & 7484 & 82 \\
\hline H65B & 9132 & 8454 & 7220 & 82 \\
\hline H65C & 9328 & 7358 & 7011 & 82 \\
\hline H66 & 9365 & 7532 & 6222 & 32 \\
\hline H67A & 9196 & 7196 & 5482 & 48 \\
\hline H67B & 8836 & 8307 & 5581 & 48 \\
\hline H67C & 8261 & 7343 & 5522 & 48 \\
\hline H68A & 9051 & 5811 & 6028 & 62 \\
\hline H68B & 8141 & 6037 & 6131 & 62 \\
\hline $\mathrm{H} 68 \mathrm{C}$ & 8779 & 6070 & 6516 & 62 \\
\hline
\end{tabular}


Table S24. Torsion angles $\left[{ }^{\circ}\right]$ for jonlm38 (3a').

\begin{tabular}{|c|c|c|c|}
\hline N1-B1-C3-C8 & $-7.9(8)$ & $\mathrm{C} 20-\mathrm{C} 15-\mathrm{C} 16-\mathrm{C} 17$ & $1.2(10)$ \\
\hline C9-B1-C3-C8 & $109.9(7)$ & B1-C15-C16-C17 & $-174.0(6)$ \\
\hline C15-B1-C3-C8 & $-121.8(6)$ & $\mathrm{C} 15-\mathrm{C} 16-\mathrm{C} 17-\mathrm{C} 18$ & $-1.0(11)$ \\
\hline N1-B1-C3-C4 & $172.0(6)$ & C16-C17-C18-C19 & $0.1(11)$ \\
\hline C9-B1-C3-C4 & $-70.1(7)$ & $\mathrm{C} 17-\mathrm{C} 18-\mathrm{C} 19-\mathrm{C} 20$ & $0.4(11)$ \\
\hline C15-B1-C3-C4 & $58.2(8)$ & $\mathrm{C} 18-\mathrm{C} 19-\mathrm{C} 20-\mathrm{C} 15$ & $-0.1(11)$ \\
\hline $\mathrm{C} 8-\mathrm{C} 3-\mathrm{C} 4-\mathrm{C} 5$ & $-1.7(11)$ & $\mathrm{C} 16-\mathrm{C} 15-\mathrm{C} 20-\mathrm{C} 19$ & $-0.7(10)$ \\
\hline $\mathrm{B} 1-\mathrm{C} 3-\mathrm{C} 4-\mathrm{C} 5$ & $178.4(7)$ & B1-C15-C20-C19 & $174.5(6)$ \\
\hline $\mathrm{C} 3-\mathrm{C} 4-\mathrm{C} 5-\mathrm{C} 6$ & $1.4(13)$ & C23-P1-C21-C22 & $95.6(5)$ \\
\hline $\mathrm{C} 4-\mathrm{C} 5-\mathrm{C} 6-\mathrm{C} 7$ & $0.4(12)$ & $\mathrm{C} 26-\mathrm{P} 1-\mathrm{C} 21-\mathrm{C} 22$ & $-153.6(5)$ \\
\hline $\mathrm{C} 5-\mathrm{C} 6-\mathrm{C} 7-\mathrm{C} 8$ & $-1.7(11)$ & Pd1-P1-C21-C22 & $-29.5(5)$ \\
\hline $\mathrm{C} 4-\mathrm{C} 3-\mathrm{C} 8-\mathrm{C} 7$ & $0.3(10)$ & $\mathrm{P} 1-\mathrm{C} 21-\mathrm{C} 22-\mathrm{P} 2$ & $37.1(6)$ \\
\hline $\mathrm{B} 1-\mathrm{C} 3-\mathrm{C} 8-\mathrm{C} 7$ & $-179.7(6)$ & $\mathrm{C} 32-\mathrm{P} 2-\mathrm{C} 22-\mathrm{C} 21$ & $95.0(5)$ \\
\hline $\mathrm{C} 6-\mathrm{C} 7-\mathrm{C} 8-\mathrm{C} 3$ & $1.4(10)$ & $\mathrm{C} 29-\mathrm{P} 2-\mathrm{C} 22-\mathrm{C} 21$ & $-153.2(4)$ \\
\hline N1-B1-C9-C10 & $-23.1(9)$ & $\mathrm{Pd} 1-\mathrm{P} 2-\mathrm{C} 22-\mathrm{C} 21$ & $-27.9(5)$ \\
\hline C3-B1-C9-C10 & $-139.3(7)$ & $\mathrm{C} 26-\mathrm{P} 1-\mathrm{C} 23-\mathrm{C} 25$ & $72.4(5)$ \\
\hline C15-B1-C9-C10 & $90.8(8)$ & $\mathrm{C} 21-\mathrm{P} 1-\mathrm{C} 23-\mathrm{C} 25$ & $-175.7(5)$ \\
\hline N1-B1-C9-C14 & $161.3(6)$ & $\mathrm{Pd} 1-\mathrm{P} 1-\mathrm{C} 23-\mathrm{C} 25$ & $-55.6(5)$ \\
\hline C3-B1-C9-C14 & $45.1(8)$ & $\mathrm{C} 26-\mathrm{P} 1-\mathrm{C} 23-\mathrm{C} 24$ & $-162.8(5)$ \\
\hline C15-B1-C9-C14 & $-84.8(8)$ & $\mathrm{C} 21-\mathrm{P} 1-\mathrm{C} 23-\mathrm{C} 24$ & $-50.9(6)$ \\
\hline C14-C9-C10-C11 & $1.2(13)$ & $\mathrm{Pd} 1-\mathrm{P} 1-\mathrm{C} 23-\mathrm{C} 24$ & $69.2(5)$ \\
\hline B1-C9-C10-C11 & $-174.7(8)$ & C23-P1-C26-C28 & $57.1(6)$ \\
\hline C9-C10-C11-C12 & $0.7(15)$ & C21-P1-C26-C28 & $-54.2(6)$ \\
\hline $\mathrm{C} 10-\mathrm{C} 11-\mathrm{C} 12-\mathrm{C} 13$ & $-2.1(14)$ & Pd1-P1-C26-C28 & $-174.1(4)$ \\
\hline C11-C12-C13-C14 & $1.7(13)$ & $\mathrm{C} 23-\mathrm{P} 1-\mathrm{C} 26-\mathrm{C} 27$ & $-176.3(5)$ \\
\hline C10-C9-C14-C13 & $-1.7(11)$ & $\mathrm{C} 21-\mathrm{P} 1-\mathrm{C} 26-\mathrm{C} 27$ & $72.4(5)$ \\
\hline B1-C9-C14-C13 & $174.3(6)$ & Pd1-P1-C26-C27 & $-47.6(5)$ \\
\hline $\mathrm{C} 12-\mathrm{C} 13-\mathrm{C} 14-\mathrm{C} 9$ & $0.3(12)$ & C22-P2-C29-C31 & $-55.3(6)$ \\
\hline N1-B1-C15-C16 & $81.4(7)$ & C32-P2-C29-C31 & $56.7(7)$ \\
\hline C3-B1-C15-C16 & $-164.4(6)$ & Pd1-P2-C29-C31 & $-175.9(5)$ \\
\hline C9-B1-C15-C16 & $-34.6(8)$ & $\mathrm{C} 22-\mathrm{P} 2-\mathrm{C} 29-\mathrm{C} 30$ & $70.9(6)$ \\
\hline N1-B1-C15-C20 & $-93.5(7)$ & C32-P2-C29-C30 & $-177.0(5)$ \\
\hline C3-B1-C15-C20 & $20.7(8)$ & Pd1-P2-C29-C30 & $-49.7(6)$ \\
\hline C9-B1-C15-C20 & $150.5(6)$ & $\mathrm{C} 22-\mathrm{P} 2-\mathrm{C} 32-\mathrm{C} 33$ & $-73.1(5)$ \\
\hline
\end{tabular}




\begin{tabular}{|c|c|c|c|}
\hline C29-P2-C32-C33 & $174.3(5)$ & N2-B2-C49-C50 & $83.4(7)$ \\
\hline Pd1-P2-C32-C33 & $46.0(5)$ & $\mathrm{C} 43-\mathrm{B} 2-\mathrm{C} 49-\mathrm{C} 50$ & $-33.2(8)$ \\
\hline C22-P2-C32-C34 & $163.6(5)$ & C37-B2-C49-C50 & $-161.6(6)$ \\
\hline C29-P2-C32-C34 & $51.0(6)$ & C54-C49-C50-C51 & $0.1(10)$ \\
\hline Pd1-P2-C32-C34 & $-77.4(6)$ & B2-C49-C50-C51 & $-174.0(7)$ \\
\hline N2-B2-C37-C42 & $163.4(6)$ & C49-C50-C51-C52 & $-2.5(12)$ \\
\hline C43-B2-C37-C42 & $-78.0(7)$ & C50-C51-C52-C53 & $3.5(12)$ \\
\hline C49-B2-C37-C42 & $49.1(8)$ & C51-C52-C53-C54 & $-2.1(11)$ \\
\hline N2-B2-C37-C38 & $-17.3(7)$ & C50-C49-C54-C53 & $1.4(9)$ \\
\hline C43-B2-C37-C38 & $101.2(6)$ & B2-C49-C54-C53 & $175.4(6)$ \\
\hline C49-B2-C37-C38 & $-131.7(6)$ & C52-C53-C54-C49 & $-0.4(10)$ \\
\hline C42-C37-C38-C39 & $-0.1(9)$ & C60-P3-C55-C56 & $-154.9(5)$ \\
\hline B2-C37-C38-C39 & $-179.3(6)$ & C57-P3-C55-C56 & $95.6(5)$ \\
\hline C37-C38-C39-C40 & $-0.3(10)$ & Pd2-P3-C55-C56 & $-28.7(5)$ \\
\hline C38-C39-C40-C41 & $1.3(10)$ & P3-C55-C56-P4 & $36.6(6)$ \\
\hline C39-C40-C41-C42 & $-2.0(12)$ & C63-P4-C56-C55 & $-154.4(5)$ \\
\hline C40-C41-C42-C37 & $1.7(12)$ & C66-P4-C56-C55 & $94.0(5)$ \\
\hline C38-C37-C42-C41 & $-0.6(10)$ & Pd2-P4-C56-C55 & $-27.9(5)$ \\
\hline B2-C37-C42-C41 & $178.7(7)$ & C60-P3-C57-C58 & $-159.8(5)$ \\
\hline N2-B2-C43-C48 & $156.3(5)$ & C55-P3-C57-C58 & $-49.9(6)$ \\
\hline C37-B2-C43-C48 & $39.2(7)$ & Pd2-P3-C57-C58 & $70.0(6)$ \\
\hline C49-B2-C43-C48 & $-89.3(7)$ & C60-P3-C57-C59 & $75.2(6)$ \\
\hline N2-B2-C43-C44 & $-26.8(8)$ & C55-P3-C57-C59 & $-174.9(5)$ \\
\hline C37-B2-C43-C44 & $-144.0(6)$ & Pd2-P3-C57-C59 & $-55.0(6)$ \\
\hline C49-B2-C43-C44 & $87.5(7)$ & C55-P3-C60-C61 & $78.8(6)$ \\
\hline C48-C43-C44-C45 & $2.5(10)$ & C57-P3-C60-C61 & $-171.0(5)$ \\
\hline B2-C43-C44-C45 & $-174.6(7)$ & Pd2-P3-C60-C61 & $-41.8(6)$ \\
\hline C43-C44-C45-C46 & $-1.4(12)$ & C55-P3-C60-C62 & $-45.9(6)$ \\
\hline $\mathrm{C} 44-\mathrm{C} 45-\mathrm{C} 46-\mathrm{C} 47$ & $-0.3(12)$ & C57-P3-C60-C62 & $64.2(6)$ \\
\hline C45-C46-C47-C48 & $0.7(11)$ & Pd2-P3-C60-C62 & $-166.6(4)$ \\
\hline C46-C47-C48-C43 & $0.6(10)$ & C56-P4-C63-C65 & $-54.4(6)$ \\
\hline C44-C43-C48-C47 & $-2.1(9)$ & C66-P4-C63-C65 & $57.3(6)$ \\
\hline B2-C43-C48-C47 & $175.0(6)$ & Pd2-P4-C63-C65 & $-175.4(5)$ \\
\hline N2-B2-C49-C54 & $-90.3(7)$ & C56-P4-C63-C64 & $72.5(5)$ \\
\hline C43-B2-C49-C54 & $153.2(5)$ & C66-P4-C63-C64 & $-175.7(5)$ \\
\hline C37-B2-C49-C54 & $24.7(8)$ & Pd2-P4-C63-C64 & $-48.4(6)$ \\
\hline
\end{tabular}


C63-P4-C66-C67

C56-P4-C66-C67

Pd2-P4-C66-C67

C63-P4-C66-C68

C56-P4-C66-C68

Pd2-P4-C66-C68
172.6(4)

$-75.3(5)$

43.1(4)

49.5(6)

161.7(5)

$-79.9(5)$ 


\section{REFERENCES:}

(1) Characterization of $\mathbf{2 a}$ is consistent with analogous (dippe) $\mathrm{Ni}\left(\mathrm{CH}_{3} \mathrm{CN}\right)$ complex. (a) García, J. J.; Arevalo, A.; Brunkan, N. M.; Jones, W. D. Organometallics 2004, 23, 3997 - 4002. (b) (a) Ateşin, T. A.; Li, T.; Lachaize, S.; Brennessel, W. W.; García, J. J.; Jones, W. D. J. Am. Chem. Soc. 2007, 129, 7562- 7569.

(2) (a) Atesin, A. T.; Oster, S. S.; Skugrud, K.; Jones, W. D. Inorg. Chim. Acta 2006, 359, 2798-2805. (b) Munjanja, L.; Brennessel, W. W.; Jones, W. D. Organometallics 2015, 34, 1716-1724. (c) Munjanja, L.; Brennessel, W. W.; Jones, W. D. Organometallics 2015, 34, 4574 4580. 